Foundations and Trends ${ }^{\circledR}$ in Systems and Control

Vol. XX, No. XX (2017) 1-207

(C) 2017 C. Prieur, I. Queinnec, S. Tarbouriech, L. Zaccarian

DOI: 10.1561/XXXXXXXXXX

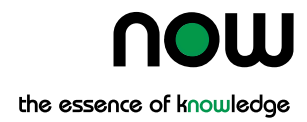

\title{
Analysis and synthesis of reset control systems
}

\author{
Christophe Prieur \\ GIPSA-lab \\ Isabelle Queinnec \\ LAAS-CNRS \\ christophe.prieur@gipsa-lab.fr \\ queinnec@laas.fr \\ Sophie Tarbouriech \\ Luca Zaccarian \\ LAAS-CNRS \\ LAAS-CNRS and \\ tarbour@laas.fr \\ University of Trento \\ zaccarian@laas.fr
}




\section{Contents}

\begin{tabular}{|ll|}
\hline Background & 3
\end{tabular}

\begin{tabular}{lll}
\hline & Introduction & 4
\end{tabular}

1.1 Historical overview . . . . . . . . . . . . . . . . . . . . 4

1.2 The Clegg integrator circuit . . . . . . . . . . . . . . . . 7

1.3 Modelling issues with reset control . . . . . . . . . . . . 10

1.4 Using thin jump sets: existence of solutions and robustness 12

1.5 An overview of recent reset systems results. . . . . . . . 16

\begin{tabular}{lll}
\hline 2 & A first planar closed loop & $\mathbf{1 8}$
\end{tabular}

2.1 Hybrid representation for solutions . . . . . . . . . . . . . 18

2.2 A motivating example . . . . . . . . . . . . . . . . . . . 21

2.3 An overview of this survey . . . . . . . . . . . . . . 24

\begin{tabular}{|ll}
\hline II Non-planar reset systems & 27
\end{tabular}

3 Nonlinear extensions of Clegg's integrator 28

3.1 A generalization of the FORE reset rule . . . . . . . . . . 28

3.2 Hybrid stabilization with reset loops: nonlinear case . . . . 31

3.3 A nonlinear control system stabilized by adding a hybrid loop 35 
3.4 Hybrid stabilization with reset loops: linear case . . . . . . 37

3.5 Examples of linear systems involving hybrid loops . . . . . 43

4 Dwell-time logic and observer-based hybrid loops 52

4.1 Overcoming two drawbacks of the hybrid loops suggested

in Chapter $3 \ldots \ldots \ldots \ldots$. . . . . . . . . . . 52

4.2 Controller architecture and output feedback stabilization . 56

4.3 Simulations $\ldots \ldots \ldots \ldots$. . . . . . . . . . . . . 67

\begin{tabular}{lll}
\hline 4.4 & Fundamental properties of temporally regularized systems & 73
\end{tabular}

5 LMI-based stability and performance analysis $\quad 80$

5.1 Introducing continuous-time $\mathcal{L}_{2}$ gains . . . . . . . 80

5.2 Lyapunov-based $t$ - $\mathcal{L}_{2}$ stability conditions . . . . . . . . . . 84

5.3 LMl-based $\mathcal{L}_{2}$ stability conditions . . . . . . . . . . . . . . 89

5.4 Application to SISO control loops with FORE . . . . . . . 92

5.5 Numerical examples . . . . . . . . . . . . . . . . . . . . 103

$\begin{array}{lll}6 & \text { Towards reset } \mathcal{H}_{\infty} \text { control design } & 111\end{array}$

6.1 Overview . . . . . . . . . . . . . . . . . . . . . . . 111

6.2 Full state availability . . . . . . . . . . . . . . . . . 112

6.3 Partial state availability . . . . . . . . . . . . . . . . . . . 119

6.4 Illustrative examples . . . . . . . . . . . . . . . . . . . . . 120

\begin{tabular}{lll}
\hline III & Planar reset systems & 129
\end{tabular}

7 Planar SISO systems with First Order Reset Elements $\quad 130$

7.1 A modified model of FORE and its Lyapunov implications. 130

7.2 Necessary and sufficient conditions for exponential stability 134

7.3 Extension to minimum phase relative degree one linear SISO plants . . . . . . . . . . . . . . . . . 140

7.4 Set-point regulation . . . . . . . . . . . . . . . . . . . . . . 144

7.5 Reference tracking . . . . . . . . . . . . . . . . . . . . . . 153

8 FORE control of an EGR valve 156

8.1 Overview . . . . . . . . . . . . . . . . . . . 156

8.2 Model parameters identification . . . . . . . . . . . . 157 
iv

8.3 Proposed regulation scheme . . . . . . . . . . . . . . . . . . 160

8.4 Results with adaptive feedforward. . . . . . . . . . . . . . 161

8.5 Results with parametrized feedforward . . . . . . . . . . . . 164

8.6 Tracking a time-varying position reference . . . . . . . . . . 167

8.7 Comparison to time-optimal and Pl solutions . . . . . . . 168

8.8 Implementation on the Diesel engine testbench . . . . . . 171

8.9 Experimental results . . . . . . . . . . . . . . . . . . 174

9 FORE Control of an electromechanical valve 179

9.1 Overview . . . . . . . . . . . . . . . . . . . . . . . . . . . . . 179

9.2 Application to the valve model and simulation results . . . 182

9.3 Experimental tests . . . . . . . . . . . . . . . . . . . 187

10 Conclusions and perspectives 191

10.1 Conclusions . . . . . . . . . . . . . . . . . . . . 191

10.2 Perspectives . . . . . . . . . . . . . . . . . . . 192

$\begin{array}{ll}11 \text { Acknowledgement } & 195\end{array}$

\begin{tabular}{ll}
\hline References & 197
\end{tabular} 


\section{Abstract}

This survey paper overviews a large core of research results produced by the authors in the past decade about reset controllers for linear and nonlinear plants. The corresponding feedback laws generalize classical dynamic controllers because of the interplay of mixed continuous/discrete dynamics. The obtained closed-loop system falls then within the category of hybrid dynamical systems, with the specific feature that the hybrid nature arises from the nature of the controller, rather than the nature of the plant, which is purely continuous-time. Due to this fact, the presented results focus on performance and stability notions that prioritize continuous-time evolution as compared to the discrete-time one. Dwell-time logics are indeed enforced on solutions, to ensure that the continuous evolution of solutions is complete (no Zeno solutions occur).

After presenting a historical motivation and an overview of the results on this topic in Part I, several results on stability and performance analysis and on control design for general linear continuous-time plants are developed in Part II. These results are developed by exploiting the well established formalism for nonlinear hybrid dynamical systems introduced by Andy Teel and co-authors around 2004. With this formalism, by ensuring sufficient regularity of the reset controller dynamics, we ensure robustness of stability with respect to small disturbances and uncertainties together with suitable continuity of solutions, generally regarded as well-posedness of the hybrid closed loop. Throughout Part II, we provide several simulation studies showing that reset control strategies may allow to attain better performance with respect to the optimal ones obtained by classical continuous-time controllers.

Finally, In Part III we focus on planar systems, that is reset closed loops involving a one dimensional linear plant and a one dimensional reset controller. For this simple interconnection interesting stability conditions can be drawn and relevant extensions addressing the reference tracking problem are introduced, illustrating them on a few relevant 
case studies emerging in the automotive field.

C. Prieur, I. Queinnec, S. Tarbouriech, L. Zaccarian. Analysis and synthesis of reset control systems. Foundations and Trends ${ }^{\circledR}$ in Systems and Control, vol. XX, no. XX, pp. 1-207 2017.

DOI: $10.1561 / \mathrm{XXXXXXXXXX}$. 
Part I

\section{Background}


1

Introduction

\subsection{Historical overview}

Reset controllers were proposed for the first time more than 50 years ago by Clegg 1958, with the aim at providing more flexibility in linear controller designs and at potentially removing fundamental performance limitations of linear controllers. The first systematic designs for reset controllers were reported in the 1970's by Krishnan and Horowitz [1974], Horowitz and Rosenbaum [1975] and there has been a renewed interest in this class of systems in the late 1990's with Beker et al. 1999b a, 2001b, 2004, Chait and Hollot 2002, Chen et al. 2000a b. 2001, Haddad et al. [2000], Hollot et al. [1997, 2001], Hu et al. [1997], Zheng et al. [2000].

More specifically, a reset controller is a linear controller whose output is reset to zero whenever its input and output satisfy an appropriate algebraic relationship. For instance, in Beker et al. 2004 and the references cited therein, a class of reset controllers was considered where the output of the controller is reset to zero whenever its input is equal to zero. The Clegg integrator introduced in Clegg [1958] acts like a linear integrator whenever its input and output have the same sign and it resets its output to zero otherwise (see Section 1.2). Con- 
sequently, its describing function has the same magnitude plot as the linear integrator but it has $51.9^{\circ}$ less phase lag. This feature of the Clegg integrator was used for the first time in Krishnan and Horowitz [1974 to provide a systematic procedure for controller design exploiting this device. Subsequently, a new reset device called the First Order Reset Element (FORE) was introduced in Horowitz and Rosenbaum 1975, essentially generalizing the Clegg integrator's base linear dynamics by also allowing for a nonzero real pole. Horowitz and Rosenbaum 1975 also proposed a design procedure consisting of two steps. First, a nonreset part of the controller was designed to achieve all design specifications except for the overshoot. Then, in the second step, the pole of the FORE was selected to reduce the overshoot. It was illustrated through examples and simulations that the controller in the first step of the procedure could indeed be designed with lower phase margin, which provided more design flexibility. A nice account of these results and their relation to more recent developments in reset control is given in Chait and Hollot 2002.

The first example that clearly illustrated the advantages of reset over linear controllers was presented in Beker et al. 2001a where a reset controller was designed to achieve design specifications that are impossible to achieve by any linear controller (see also Feuer et al. [1997]). Indeed, for linear plants including an integral action, if the desired rise time is sufficiently small, then the output must overshoot with any linear controller. However, a reset controller is designed in Beker et al. 2001a] that overcomes this fundamental performance limitation of linear controllers. To date, this appears to be the only real situation where reset designs have been shown to outperform the best possible classical design, nevertheless, practical experience reveals that desirable closed-loop behavior is obtained when suitably embedding resets in otherwise continuous-time control devices. Examples of such experiences can be found, e.g., in the experimental applications reported in Zheng et al. 2007, Fernandez et al. 2008, Wu et al. [2007], Li et al. [2009], Bakkeheim et al. [2008], Guo et al. [2009], Panni et al. [2014], Cordioli et al. [2015], Zheng et al. [2000], Carrasco and Baños [2012], Li et al. [2011], Vidal and Baños [2010], Villaverde et al. [2011]. The 
difficulty in proving rigorous statements with reset systems was due to the lack of suitable stability and performance analysis tools for systems whose solutions may experience instantaneous jumps (this is the case for an integrator reset to zero).

From a theoretical viewpoint, first attempts to rigorously analyze stability of reset systems with Clegg integrators can be found in $\mathrm{Hu}$ et al. [1997, Hollot et al. [1997]. In particular, an integral quadratic constraint was proposed in Hollot et al. 1997 to analyze stability of a class of reset systems. However, the proposed condition was conservative as it was independent of reset times. BIBO stability analysis of reset systems consisting of a second order plant and a FORE was conducted in Chen et al. [2001] (see also Chen et al. [2000b]). The proofs are based on an explicit characterization of reset times which are proved to be equidistant under mild conditions. Using this fact, the authors prove asymptotic and BIBO stability of the reset system via the discrete-time model of the system that describes the system at reset times only. However, the same approach could not be used to analyze higher order reset systems. Stability analysis of general reset systems can be found in Beker et al. [2004] (see also Hollot et al. [2001, Chen et al. 2000a ) where Lyapunov based conditions for asymptotic stability were presented and computable conditions for quadratic stability based on linear matrix inequalities (LMIs) were given. Moreover, in Beker et al. [2004], BIBO stability of general reset systems was obtained as a consequence of quadratic stability and an internal model principle was proved for reference tracking and disturbance rejection.

In the last decade, perhaps triggered by the inspiring work of Beker et al. 2004, a significant renewed interest in Lyapunov-based analysis and synthesis for reset systems has been witnessed by the scientific community. This survey paper reports on a research strand that started around 2005, motivated by the results in Beker et al. [2004], wherein some recent stability and performance analysis tools for hybrid dynamical systems have been brought to bear into the framework of reset control systems. While many alternative and relevant approaches have been developed during the last decade (a selection of them is briefly overviewed in the following Section 1.5), we specifically concentrate 
here on a research strand that emerged from the hybrid Lyapunov theory proposed in Goebel et al. [2012]. Some important differences between what is reported here and alternative approaches is discussed in the next sections, which explain the spirit of our approach to reset control. To this end, we need to somewhat come back to the very origins of reset control and take a close look at the analog circuit proposed by J.C. Clegg in 1958, which is discussed in the next section.

\subsection{The Clegg integrator circuit}

In 1958, J.C. Clegg published a paper (Clegg [1958|) where he proposed a modification to the existing analog control schemes to reduce the phase lag induced by a linear integrator. The relevance of Clegg's work was mostly targeted to analog control, because digital control systems were still non-existent in the late 1950's, nevertheless, follow-up works considered digital versions of the scheme proposed by Clegg. Let us here consider the analog device proposed by Clegg and derive the corresponding equations.

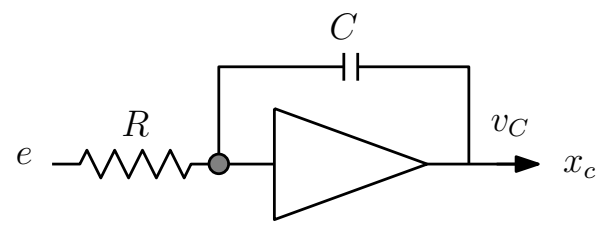

Figure 1.1: A linear analog integrator.

In the ideal case of using infinite gain operational amplifiers, it is well known that a linear integrator can be implemented using a resistor on the input path and a capacitor on the feedback path of the circuit, as represented in Figure 1.1. The corresponding input/output relation of the linear integrator can be written in the time domain as $\dot{x}_{c}=-\frac{1}{R C} e$, where we use $x_{c}$ for the integrator output, to resemble the fact that the integrator state is the state of a feedback controller from the tracking error $e$.

The modification proposed by Clegg corresponds to the scheme of Figure 1.2 (which is reported here from Clegg [1958] with a sign in- 


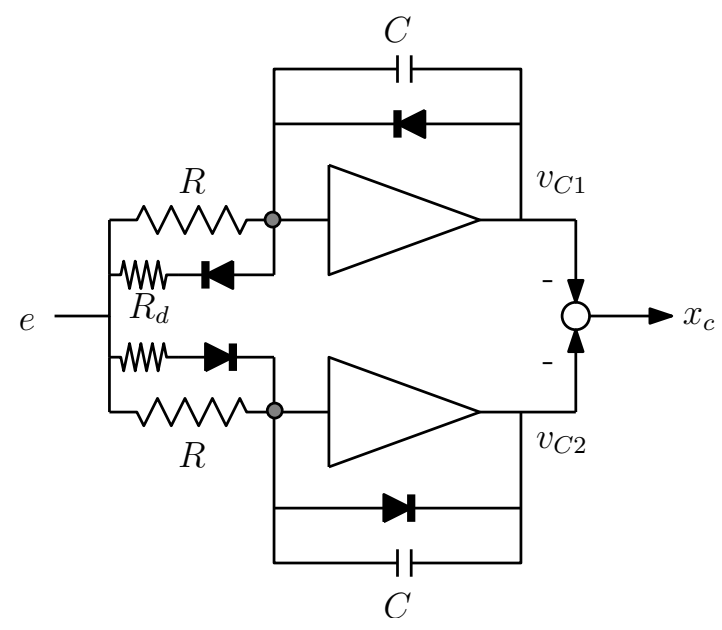

Figure 1.2: The "Clegg integrator".

version at the output, for convenience of exposition). We describe the Clegg integrator dynamics assuming that $R_{d} \ll R$ and disregarding the forward-bias voltage drop across the diodes, leading to the following ideal relationships between voltage and current across capacitors, resistors and diodes:

$$
\begin{aligned}
& i_{C}(t)=C \frac{d v_{C}(t)}{d t} \\
& i_{R}(t)=v_{R}(t) / R \\
& i_{D}(t)=\left\{\begin{aligned}
+\infty, & \text { if } v_{D}(t)>0, \\
0 & \text { if } v_{D}(t) \leq 0 .
\end{aligned}\right.
\end{aligned}
$$

First note that by the infinite gain assumption of the operational amplifier, the input voltages (marked by gray dots on the figure) are always zero. Then, the two capacitors' voltages satisfy $v_{C 1}(t) \leq 0$ and $v_{C 2}(t) \geq 0$ for all times (otherwise the infinite current flowing in the diodes would instantaneously discharge the capacitor). Moreover, when $e(t)<0$, regardless of the preceding voltage stored in the upper capacitor, the current flowing in the two diodes and through the upper $R_{d}$ will (almost) instantaneously impose $v_{C 1}(t)=0$. However, when $e(t) \geq 0$, the upper circuit will correspond to the linear integrator because the 
diodes will both be open (being subjected to a non-positive voltage). Similarly for the lower circuit, if $e(t)>0$, we will have $v_{C 2}(t)=0$ and if $u(t) \leq 0$ the circuit will integrate. Since, as commented above, $v_{C 1}(t) \leq 0$ and $v_{C 2}(t) \geq 0$ for all times, given $x_{c}(t):=-v_{C 1}(t)-v_{C 2}(t)$, the integrating and reset conditions for both circuits can be written as the following hybrid dynamics

$$
\begin{cases}\dot{x}_{c}=\frac{1}{R C} e, & \text { is allowed when } x_{c} e \geq 0, \\ x_{c}^{+}=0, & \text { is allowed when } x_{c} e \leq 0,\end{cases}
$$

where we insist on the fact that the situation $e(t)=0$ leads to an undetermined behavior of the circuit mostly dependent on the effect of unmodeled noise and uncertainties. In this model, we use the shorthand notation $\dot{x}$ for $\frac{d}{d t} x(t, j)$ and $x^{+}$for $x(t, j+1)$ which will be formally defined later on in the survey. The strategy that we prefer to adopt for handling such undetermined cases is to allow for multiple solutions to the dynamics (1.1) thereby considering in our model all the possible scenarios. See the discussion given later in Remark 1.1.

One way to understand the hybrid model (1.1) for the Clegg integrator is to call its first equation the "flow" equation and its second equation the "jump" equation. The two conditions at the right hand side become then the "flow" condition and the "jump" condition. At any time, a solution to the hybrid system (1.1) may then flow or jump depending on whether its value at that time belongs to the so-called "jump set" (namely, the set of states for which the jump condition is true) or it belongs to the "flow set". In case both conditions are true, then the solution will be free to choose whether flowing or jumping, thereby establishing a peculiar non-uniqueness feature.

Further insight on equation (1.1) can be gained by observing that $e$ and $x_{c}$ can never have opposite signs. Indeed, if $e \geq 0$, then $v_{C 2}^{+}=0$, and since $v_{C 1} \leq 0$ for all times, $e x_{c} \geq 0$. Similarly for the case $e \leq 0$. On the other hand, whenever $e \neq 0$, there will always be one circuit integrating (the upper one if $e>0$ and the lower one if $e<0$ ) and the other circuit will be forced to be at zero. For illustrative purposes, Figure 1.3 represents the Clegg integrator state (solid curve) when the input $e$ is selected as a sine wave with unit frequency (dashed curve). 


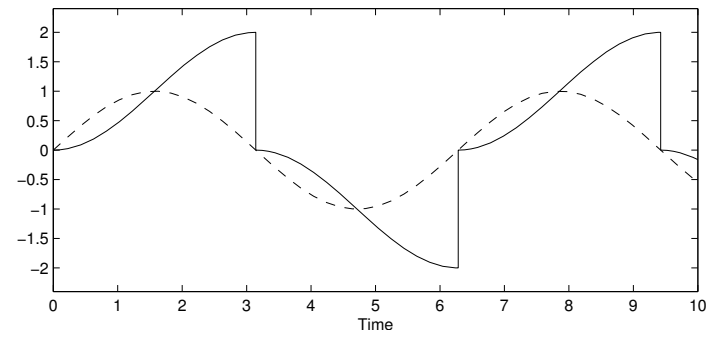

Figure 1.3: Response of the Clegg integrator (solid) to a sine input (dashed).

Note that $x_{c}$ and $e$ always have the same sign.

One way to interpret the dynamics 1.1 is to regard it as a linear filter with a pole at the origin embedded with a special resetting rule dependent on the value of the input and output of the filter at each time. This interpretation is the starting point for the FORE generalization discussed in the next section.

\subsection{Modelling issues with reset control}

The model (1.1) derived in the previous section for the hybrid dynamics can be easily generalized to the following dynamics, where the eigenvalue associated to the continuous dynamics is not necessarily at zero:

$$
\begin{cases}\dot{x}_{c}=a_{c} x_{c}+b_{c} e, & \text { is allowed when } x_{c} e \geq 0, \\ x_{c}^{+}=0, & \text { is allowed when } x_{c} e \leq 0,\end{cases}
$$

the model of the Clegg integrator corresponding to $a_{c}=0$ and $b_{c}=\frac{1}{R C}$. The generalized dynamical system $(1.2)$ was introduced in Horowitz and Rosenbaum 1975 and therein called First Order Reset Element (FORE). In Horowitz and Rosenbaum 1975] and follow-up works, this generalization was meant for stable filters (namely $a_{c} \leq 0$ ), but it will be emphasized in this survey that this is not a necessary assumption and indeed unstable selections $\left(a_{c}>0\right)$ lead sometimes to desirable aggressive control actions.

It should be acknowledged that models 1.1$)$ and $(1.2)$ do not correspond to the models originally developed from the 1960s. Indeed, while 


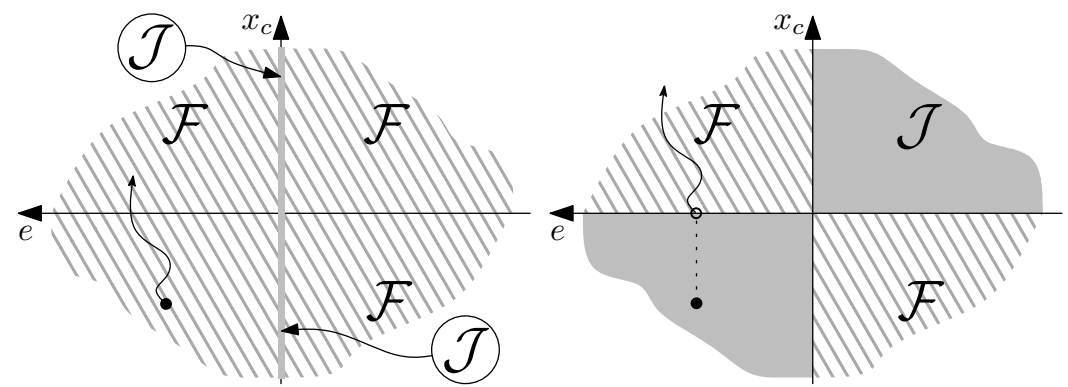

Figure 1.4: The jump (grey) and flow (striped) sets for the model 1.3 (left), and original model 1.2 (right) proposed by Clegg.

Clegg's discussion in Clegg [1958] well referred to the dynamical behavior of his analog circuit as a circuit resetting to zero whenever input and output had opposite signs, his qualitative description incorporated the following observation:

"Whenever the input voltage $e$ passes through zero from either direction, the output voltage is quickly dropped to zero."

This sentence propagated into the follow-up work of Horowitz and coauthors, who never really wrote down equations but only described in words this behavior specifying resetting the controller state to zero at zero-crossings of the input. Much later in the 1990s, Hollot and coauthors (see, e.g., Chait and Hollot [2002, , Beker et al. [2004] and references therein), and then also Baños and co-authors (see, e.g., Baños and Barreiro 2011] and references therein) reported the following dynamical description of the Clegg mechanism:

$$
\begin{cases}\dot{x}_{c}=a_{c} x_{c}+b_{c} e, & \text { if } e \neq 0, \\ x_{c}^{+}=0, & \text { if } e=0 .\end{cases}
$$

The modified hybrid dynamics (1.3) was then used as the baseline hybrid reset control mechanism implemented in the control logic of modern digital control systems. Somehow the nice and intrinsic robustness properties of the analog circuit proposed by Clegg got lost along the route towards digitalization of modern feedback control. In 
particular, dynamics 1.3 no longer describes the behavior of the circuit in Figure 1.2 for $a_{c}=0$ because solutions to 1.3 starting from $x_{c} \neq 0$ and $e x_{c}<0$ do not lead to an instantaneous reset to zero of the controller state $x_{c}$. In particular, dynamics $(1.3)$ is associated to resetting in a so-called "thin set", as opposed to dynamics (1.2) (and the behavior of Clegg's circuit) where resets are enforced in half of the input-output space of the controller. This difference is well highlighted in Figure 1.4 where the $e$ axis has been reversed in anticipation for negative error feedback interconnection of the FORE with a linear plant. In Figure 1.4 the sets enabling continuous flow of solutions are denoted by $\mathcal{F}$ (the "flow set") and the sets enabling discrete jump of solutions are denoted by $\mathcal{J}$ (the "jump set"). Figure 1.4 also shows the possible different evolutions of two solutions starting from the same initial conditions, for the two dynamics.

\subsection{Using thin jump sets: existence of solutions and robust- ness}

The model 1.2 for the first order reset element (FORE) was first introduced in Nešić et al. 2005, Zaccarian et al. [2005, where the hybrid dynamical systems formalism of Goebel et al. [2009, 2012] has been employed for the first time for representing the peculiar evolutions of reset control systems whose state (notably, the controller state) may be integrated following a differential equation during the continuoustime flow phase, or may be reset to zero following a discrete update law at the jump times.

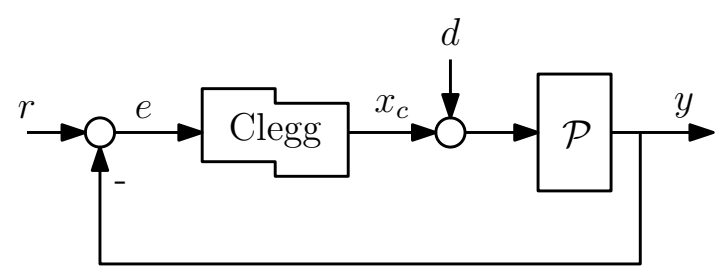

Figure 1.5: A Clegg integrator in error feedback interconnection with a linear plant.

The useful features of model 1.2 have been first characterized in 
Nešić et al. 2005, Zaccarian et al. 2005, and are worth summarizing here, with specific reference to the typical scenario of a Clegg integrator (or a more general FORE) interconnected in error feedback with a linear continuous-time plant $\mathcal{P}$, as represented in Figure 1.5. When focusing on stabilization only (that is, $r=0$ ), the general dynamics arising from using this model may be well represented by using the notation in Goebel et al. 2009, 2012, and corresponds to the following closed loop involving the overall state $x:=\left[\begin{array}{l}x_{p} \\ x_{c}\end{array}\right]$, with $x_{p}$ being the state of the plant $\mathcal{P}$ :

$$
\begin{cases}\dot{x}=A x+B d & \text { is allowed when } x \in \mathcal{F} \\ x^{+}=G x & \text { is allowed when } x \in \mathcal{J},\end{cases}
$$

where $A, G$ and $B$ are suitable constant matrices and the jump and flow sets correspond to the following symmetric cones, defined on the basis of an output equation $y=C_{y} x$, and already shown at the right of Figure 1.4 (remember that we are looking at a negative feedback interconnection that motivates reversing the horizontal axis)

$$
\left.\begin{array}{rl}
\mathcal{F}:=\left\{\left(x_{p}, x_{c}\right):\left[\begin{array}{c}
x_{c} \\
y
\end{array}\right]^{\top}\left[\begin{array}{ll}
0 & 1 \\
1 & 0
\end{array}\right]\left[\begin{array}{c}
x_{c} \\
y
\end{array}\right] \leq 0\right\}, \\
\mathcal{J}:=\left\{\left(x_{p}, x_{c}\right):\left[\begin{array}{c}
x_{c} \\
y
\end{array}\right]^{\top}\left[\begin{array}{ll}
0 & 1 \\
1 & 0
\end{array}\right]\left[\begin{array}{c}
x_{c} \\
y
\end{array}\right] \geq 0\right.
\end{array}\right\} .
$$

The overall dynamics 1.4, 1.5 falls into the larger class of homogeneous hybrid systems (see, e.g., Goebel and Teel 2010, Tuna and Teel [2006]), and will be the modeling framework adopted in this survey. We emphasize that the jump and flow sets defined in (1.5) are closed. This condition is necessary for the theoretical developments in Goebel et al., 2012, Ch. 7] to apply. Those results (which have also been used in Nešić et al. 2005, Zaccarian et al. 2005 and later works) allow us to establish existence of solutions from any initial conditions (therefore, some type of well posedness of the hybrid dynamics) in addition to suitable robustness properties of asymptotic stability of the origin for the error dynamics of the closed loop represented in Figure 1.5 .

Remark 1.1. (Uniqueness of solutions) Note that asking that the sets $\mathcal{F}$ and $\mathcal{J}$ be closed implies that there are some regions of the state 
space belonging to both sets. Therefore, solutions may jump or flow in these regions, so that the solutions to the arising reset linear systems may be non unique. Non uniqueness becomes a necessary notion when wanting to establish robust results for the reset system, as a matter of fact, when the feedback system is affected by (arbitrarily small) noise, the state could be pushed in several different directions and different solutions may correspond to different noise selections. These and other robustness issues are addressed and solved in the hybrid framework that we adopt here and in the stability results that we will rely on in this survey.

We emphasize now that the desirable existence and robustness properties highlighted above for the adopted modeling framework, are not guaranteed, in general, whenever relying on the alternative model (1.3). When using that model, and using the notation of some recent papers, the closed loop in Figure 1.5 may be represented by the equations

$$
\begin{cases}\dot{x}=A x+B d, & \text { if } x \notin \mathcal{M} \\ x^{+}=G x, & \text { if } x \in \mathcal{M},\end{cases}
$$

where $\mathcal{M}:=\left\{x: C_{y} x=0\right.$, and $\left.(I-G) x \neq 0\right\}$ (recall that we defined $\left.y=C_{y} x\right)$.

Model (1.6), which is based on (1.3), is actually used in a large number of results that can be found in the literature (see Bupp et al. [2000], Chait and Hollot [2002, Beker et al. [2004], Baños and Barreiro [2011], Barreiro et al. [2014], Ghaffari et al. [2014] just to cite a few) but is associated to some subtle issues related to existence of solutions. In particular, the following observation was already made in Nešić et al. 2005] regarding [Beker et al., 2004, Theorem 1], which establishes asymptotic stability of the origin under suitable Lyapunov conditions. Consider however the reset system 1.6 with $d=0$ and $A=\left[\begin{array}{ccc}-1 & 0 & 0 \\ 0 & -1 & -1 \\ 0 & 1 & -1\end{array}\right], G=\left[\begin{array}{lll}1 & 0 & 0 \\ 0 & 1 & 0 \\ 0 & 0 & 0\end{array}\right], C_{y}=\left[\begin{array}{lll}1 & 0 & 0\end{array}\right]$. Then it is not clear how to define solutions for an initial condition satisfying $C_{y} x_{0}=0$, $(I-G) x_{0}=0$. Indeed, in that case $x_{0} \notin \mathcal{M}$ and the reset is not possible at the initial time, which means that the dynamics can only be governed by the flow equation (1.6) for small $t \geq 0$. Moreover, integrating the differential equation (1.6) from the same initial condition yields 
$C_{y} x(t)=0$ for all $t$ and $(I-G) x(t)=\left[\begin{array}{lll}0 & 0 & x_{3}(t)\end{array}\right]^{\top}$, which is initially zero but is nonzero for all small $t$ (thus $x(t) \in \mathcal{M}$ for $t>0$ and thus flowing from the initial condition is not possible). Note that the conditions of [Beker et al., 2004, Theorem 1] hold for this example by simply selecting $V(x)=|x|^{2}$, which yields $\dot{V}=-2 V$ and $\Delta V \leq 0$. However the established stability conditions hold for a system that does not guarantee existence of solutions from some initial conditions.

Due to the reasons above, and due to the lack of guarantee of robustness of asymptotic stability, we will restrict our attention to the formalism in 1.4, 1.5 and will establish robust properties via the Lyapunov tools of Goebel et al. 2012.

Remark 1.2. It should be further emphasized that in the model 1.6 resets are only possible on the hyperplane $C_{y} x=0$ (as long as some flow has occurred since the last reset), whereas in our model (1.4), (1.5) resets are enforced on a sector $\mathcal{J}$. As a consequence, solutions to model 1.6) flow also in regions of the state space where our model does not allow flowing solutions. The consequence of this fact is that when wanting to use Lyapunov tools to prove asymptotic or exponential stability of the origin, using (1.6) it is necessary to impose a "decrease along flows" condition in almost all the state space, which then implies, by continuity, that this condition holds everywhere. Instead, with our model, and using the Lyapunov tools of Goebel et al. [2012], we only need to impose the "decrease along flows" condition in half of the state space (well understood from Figure 1.4 and this leads to less conservative conditions. In particular, even for just a Clegg integrator connected to an integrator plant $\mathcal{P}=\frac{1}{s}$ (this is the system considered in Beker et al. 2001a , corresponding to $\left.A=\left[\begin{array}{cc}0 & 1 \\ -1 & 0\end{array}\right]\right)$ the model 1.6 cannot lead to a strict Lyapunov function proving asymptotic stability of the origin. We will show in the next chapters that desirable stability properties can be shown with model 1.4, 1.5 even in cases when the continuous-time linear dynamics associated to matrix $A$ is exponentially unstable, because the stabilization is obtained by way of the resetting mechanism. This peculiar feature of stabilizing a plant by way of exponentially diverging inputs, that are eventually reset to some value leads to desirable and aggressive control actions, is well illustrated by the simulations and 
experimental studies reported in the last part of this survey.

\subsection{An overview of recent reset systems results}

In the previous section we clarified that this survey paper is focused on robust reset systems, arising from the use of model (1.1) and the arising closed-loop representation (1.4), (1.5).

This modeling framework has been used in a range of recent papers to address various analysis and design questions for reset systems. Lyapunov like conditions for $\mathcal{L}_{2}$ stability and exponential stability of reset systems have been presented in Nešić et al. [2008b]. Our conditions involve locally Lipschitz Lyapunov functions as opposed to continuously differentiable ones considered in Beker et al. 2004. This allowed us to consider piecewise quadratic Lyapunov functions in verifying exponential or $\mathcal{L}_{2}$ stability of reset systems (see Zaccarian et al. [2011]). Some explicit Lyapunov functions have been computed in Zaccarian et al. [2006], while the properties of reset set-point stabilizers and necessary and sufficient conditions for exponential and $\mathcal{L}_{2}$ stability have been reported, respectively, in Zaccarian et al. [2007] and Nešić et al. 2008a. LMI-based approaches for the $\mathcal{H}_{2}$ performance analysis and $\mathcal{L}_{2}$ performance analysis of reset control systems have been proposed, respectively, in Witvoet et al. [2007] and Aangenent et al. [2008. (see also Aangenent et al. [2010]). Solution to the $\mathcal{H}_{\infty}$ design problem has been provided in Fichera et al. [2013]. Loquen et al. 2007] addressed the presence of input saturation in reset systems while Tarbouriech et al. 2011] suggested anti-windup actions to manage input saturation effects. Finally, Loquen et al. 2008] studied stability of reset systems in the presence of nonzero reference signals.

Nevertheless, there are several recent additional results in the literature, following somewhat different routes, but indicating that reset control is yet an active research field attracting much scientific interest. One route is devoted to strategies where the reset actions are triggered at fixed time instants, often periodic. Stability and $\mathcal{L}_{2}$-gain properties have been addressed in Heemels et al. [2016] and discrete-time triggering conditions have been provided in Guo et al. [2012]. Nearly-periodic 
situations have been considered in Hetel et al. [2013. More recently, van Loon et al. 2017] proposed frequency-domain tools for stability analysis of reset control system, with the objective to attract interest of industrials for reset control strategies. In Zhao and Hua [2017], a generalized first-order reset element (GFORE) has been studied.

Another route is dedicated to reset observers, as suggested in Paesa et al. [2011, 2012], Zhao and Wang [2014]. Presence of time-varying delays has been addressed in Baños and Barreiro 2009, Barreiro and Baños [2010, Davó et al. [2017], and application to networked control system has been suggested in Baños et al. 2014a. Finally, reset logics for improving the performance of high-gain observers have been studied in Andrieu et al. [2016].

The main stream of studies relative to reset control systems has been considering interconnection of reset compensators with linear plants. There exist however a few works that explored the interconnection of reset control systems with nonlinear plants. A passivity-based approach has been proposed in Carrasco et al. [2010]. Passivation-based arguments are also used in Forni et al. 2011. Stability analysis for nonlinear systems involving some resetting action at fixed time instants was also provided in Rios et al. 2017. 


\section{2}

\section{A first planar closed loop}

\subsection{Hybrid representation for solutions}

Before even addressing the problem of characterizing useful properties of the solutions to reset linear systems (such as the Clegg integrator (1.1)), it is mandatory to adopt a mathematical representation for such solutions, which is capable of guaranteeing fundamental properties, such as existence of solutions for all times and perhaps robustness of the stability results.

A first step in this direction corresponds to suitably defining the domain of solutions for the reset system. In particular, the domain of solutions $x_{c}$ to the Clegg integrator of Figure 1.2 will be taken to be the Cartesian product of two domains: one, denoted by $t$, keeping track of the elapsed ordinary continuous time and the other one, denoted by $j$, related to the number of jumps that occurred since the initial hybrid time $\left(t_{0}, j_{0}\right)=(0,0)$. This type of mathematical framework for hybrid systems was introduced in Goebel et al. 2004 and has been first used in Nešić et al. [2005], Zaccarian et al. 2005] in the context of reset control systems.

According to the notion of solutions commented above, the Clegg 
integrator dynamics (1.1) can be written as

$$
\begin{cases}\dot{x}_{c}(t, j)=\frac{1}{R C} e(t), & \text { is allowed when } x_{c}(t, j) e(t) \geq 0, \\ x_{c}(t, j+1)=0, & \text { is allowed when } x_{c}(t, j) e(t) \leq 0,\end{cases}
$$

where $x_{c}(t, j)$ represents the state of the Clegg integrator at time $t$, also considering that $j$ resets have occurred before time $t$. Note that with this notation, if $t_{j}$ represents the time at which the $j$-th reset occurred, then $x_{c}\left(t_{j}, j-1\right)$ represents the integrator value before the jump and $x_{c}\left(t_{j}, j\right)$ represents the integrator value after the jump. With this idea in mind, one realizes that the domain of a solution is a suitable subset of $\mathbb{R}_{\geq 0} \times \mathbb{Z}_{\geq 0}$ wherein continuous and discrete-time directions $(t, j)$ never evolve backwards. Upon deciding whether jumping or flowing, each solution will then select its suitable domain.
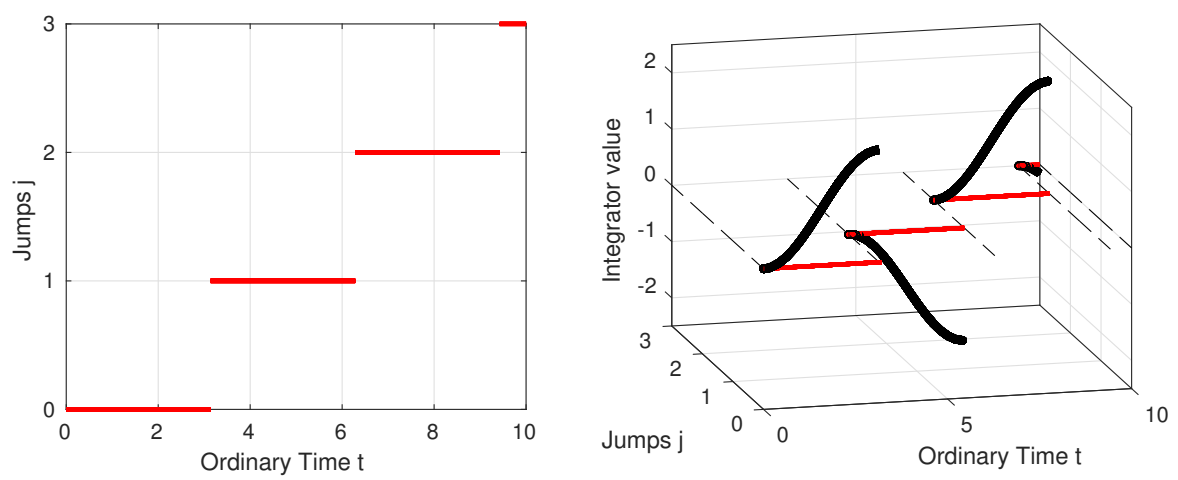

Figure 2.1: Response of Figure 1.3 in a hybrid domain.

In particular, according to the hybrid systems framework of Goebel et al. 2009, 2012, and as represented at the left of Figure 2.1, a hybrid time domain is defined as a subset of $[0, \infty) \times \mathbb{Z}_{\geq 0}$, given as a union of finitely or infinitely many intervals $\left[t_{i}, t_{i+1}\right] \times\{i\}$ where the numbers $0=t_{0}, t_{1}, \ldots$, form a finite or infinite nondecreasing sequence and $t_{1}, t_{2}, \ldots$ are called "jump times". The last interval is allowed to be of the form $\left[t_{i}, T\right)$ with $T$ finite or $T=+\infty$. As an example, Figure 2.1 represents the same response shown in Figure 1.3 with this notion of solution. The dashed horizontal lines in the right subfigure represent 
the jump times $t_{j}$ (here corresponding to $t_{1}=2.2, t_{2}=6.15$ and $\left.t_{3}=9.6\right)$ and the red lines in both plots are the hybrid domain of the solution $x_{c}$. Throughout the rest of the paper we will always use the shorthand notation $\dot{x}$ for $\dot{x}(t, j)$ and $x^{+}$for $x(t, j+1)$.

Following a similar generalization, we realize that we may represent dynamics (1.4) as a hybrid dynamical system, under the assumption that $d$ is a locally integrable signal (even though in most cases a piecewise continuous selection should be enough). Under these mild assumptions on $d$, to guarantee existence of solutions (see Goebel et al. [2009, 2012 ), it is sufficient to assume that $\mathcal{F}$ and $\mathcal{J}$ are closed sets (this is the case, for example, if one selects them as the symmetric cones in (1.5)). Then a solution $x(\cdot, \cdot)$ of the hybrid system (1.4), or its shortcut representation given by

$$
\begin{array}{ll}
\dot{x}=A x+B_{d} d, & x \in \mathcal{F} \\
x^{+}=G x, & x \in \mathcal{J},
\end{array}
$$

is a function defined on the hybrid time domain $\operatorname{dom}(x)$ such that:

(i) for all $j$ and almost all $t$ such that $(t, j) \in \operatorname{dom}(x)$, we have

$$
x(t, j) \in \mathcal{F} \text {, and } \dot{x}(t, j)=A x(t, j)+B_{d} d(t),
$$

(ii) for all $(t, j) \in \operatorname{dom}(x)$ such that $(t, j+1) \in \operatorname{dom}(x)$ we have

$$
x(t, j) \in \mathcal{J} \text { and } x(t, j+1)=G x(t, j) .
$$

Note that, following the well established notation in Goebel et al. 2012 , we dropped the sentence "is allowed when" from equations 1.4 to obtain the compact notation in (2.2).

We may now be more precise about some notation that has been already used in the previous paragraphs. We call equation (2.2a) the "flow" equation and equation (2.2b) the "jump" equation. The two conditions at the right are the "flow" condition and the "jump" condition. The solution to the hybrid system will then flow or jump depending on whether its value at that time belongs to the so-called "jump set" (namely, the set of states and inputs for which the jump condition is true) or it belongs to the "flow set" or even both. Note that if an initial state belongs to both sets then both flows and jumps are allowed, which may lead to non-unique solutions. 
While the example treated in this chapter has linear flow and jump maps, therefore is well described by the homogeneous dynamics 2.2, the solution concept introduced above also applies to a nonlinear generalization of the hybrid dynamics, corresponding to

$$
\begin{cases}\dot{x}=f(x, d), & x \in \mathcal{F} \\ x^{+}=g(x), & x \in \mathcal{J},\end{cases}
$$

for some selection of the closed sets $\mathcal{F}$ and $\mathcal{J}$. This type of generalization will be useful in Chapter 3.

\subsection{A motivating example}

To introduce and motivate the structure of this survey, it is useful to consider the simplest possible example of reset control system, which has been the subject of several studies, including the one in Beker et al. 2001a]: the case of a Clegg integrator controlling a linear plant corresponding to a single integrator, in negative error feedback. This is essentially the control scheme of Figure 1.5 with $\mathcal{P}=\frac{1}{s}$. In statespace form, which is necessary for suitably describing the nonlinear reset phenomenon, the plant dynamics is described by the differential equation $\dot{y}=x_{c}$, where $x_{c}$ is the output of the Clegg integrator in 2.1. Considering the simplest possible case of $R C=1$ in the Clegg dynamics, for a constant reference $r$, the feedback interconnection is well described in error coordinates $x:=\left(x_{p}, x_{c}\right):=\left(y-r, x_{c}\right)$ as

$$
\begin{cases}\dot{x}=\left[\begin{array}{cc}
0 & 1 \\
-1 & 0
\end{array}\right] x, & x \in \mathcal{F}=\left\{\left(x_{p}, x_{c}\right): x_{p} x_{c} \leq 0\right\} \\
x^{+}=\left[\begin{array}{ll}
1 & 0 \\
0 & 0
\end{array}\right] x, & x \in \mathcal{J}=\left\{\left(x_{p}, x_{c}\right): x_{p} x_{c} \geq 0\right\},\end{cases}
$$

where the flow and jump sets $\mathcal{F}$ and $\mathcal{J}$ exactly correspond to the two half planes represented at the left of Figure 2.3 (see also the right of Figure 1.4). The flow equation in (2.4) represents the integrator nature of both plant and controller, and the minus sign in the $(2,1)$ entry of matrix $A$ is motivated by the negative feedback interconnection. The jump equation, instead, captures the fact that $x_{p}$ does not change across jumps, whereas $x_{c}$ is reset to zero. 
The solutions of the closed loop (2.4) exhibit a peculiar behavior. Whenever they evolve in the second and fourth quadrant, they move along circles (because the continuous dynamics is governed by two complex conjugate eigenvalues at $\pm i$ ). It is quite straightforward to conclude that these flowing solutions spin in the clockwise direction in the $\left(x_{p}, x_{c}\right)$ plane as shown at the left of Figure 2.3. When they reach the vertical axis, since they cannot flow outside $\mathcal{F}$, they are forced to jump and this jump instantaneously resets them to zero. From zero, solutions may jump or flow, but regardless of their favorite (discrete or continuous) evolution, they are forced to remain in zero, which is an equilibrium, despite the peculiar non-uniqueness of solutions characterizing it.

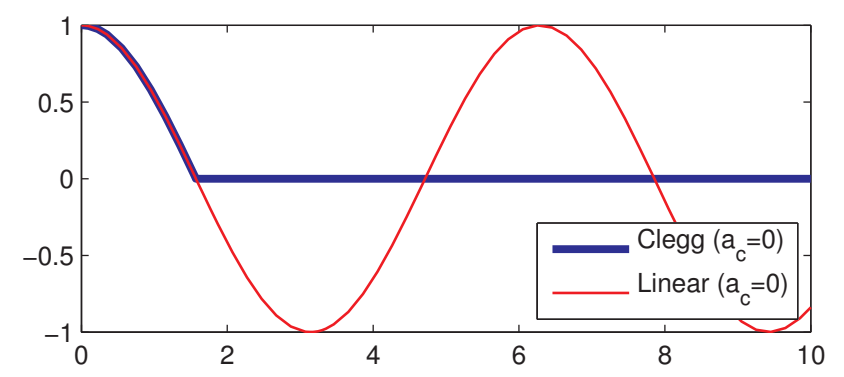

Figure 2.2: Plant output response for the motivating example, starting from $x_{c}(0,0)=0$ and $x_{p}(0,0)=1$.

Figure 2.2 shows (see the bold line) a possible solution of (2.4) starting from $\left(x_{p}(0,0), x_{c}(0,0)\right)=(1,0)$ and compares it to the solution of a purely continuous-time dynamics $\dot{x}=\left[\begin{array}{cc}0 & 1 \\ -1 & 0\end{array}\right] x$ where the controller is never reset to zero. The domain of the hybrid solution is projected on the $t$ direction to simplify the comparison to the continuous solution. The specific blue solution that we show here decides to flow indefinitely so that it can be regarded as an eventually continuous solution (it only performs one jump). While the blue solution is unique in the first portion of its time domain, until the jump time $t_{1}=\pi$ (this is the time when $x_{p}$ reaches zero), its evolution is only one among infinitely many others after this time, because from $x\left(t_{1}, 1\right)=(0,0)$, solutions 
may jump or flow at each one of the following hybrid time.
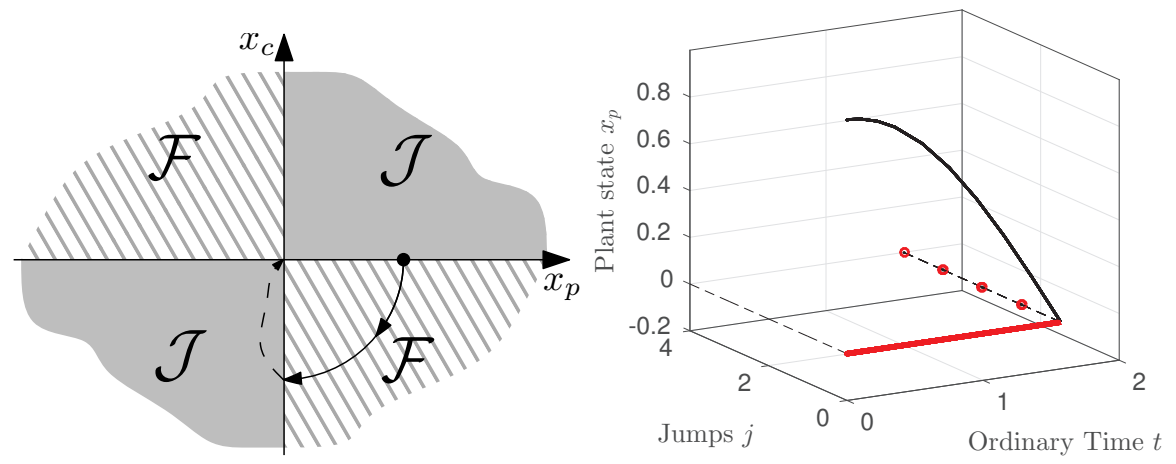

Figure 2.3: Phase-plane and hybrid representation of a solution of 2.4.

The left of Figure 2.3 shows the phase plane representation of the solution in Figure 2.2 and of all the possible other ones, because once they reach the origin they all remain there in the future (continuous or discrete, or both) evolution. The right of Figure 2.3 shows the $x_{p}$ component of one of the possible solutions, together with its time domain. That specific solution is eventually discrete and performs an infinite number of jumps never flowing again after reaching the origin.

Despite the non-uniqueness of solutions at the origin, it is rather intuitive to imagine that the following property of solutions should hold for the closed loop (2.4). The origin is globally exponentially stable for system (2.4), in the sense that there exist constants $m, \ell>0$ such that given any initial condition $x(0,0)=\left(x_{p 0}, x_{c 0}\right) \in \mathbb{R}^{2}$ all solutions $x$ satisfy

$$
|x(t, j)| \leq m e^{-\ell(t+j)}|x(0,0)|, \text { for all }(t, j) \in \operatorname{dom}(x) .
$$

When using hybrid tools to address reset control systems, it should be recognized that these control systems are generally designed for a continuous-time plant. Therefore, the continuous-time evolution of the solution is somewhat more important due to its physical meaning. Because of this reason, in this survey we will soon concentrate on alternative $t$-related definitions of exponential convergence corresponding to 
modifying the bound above as follows:

$$
|x(t, j)| \leq m e^{-\ell t}|x(0,0)|, \text { for all }(t, j) \in \operatorname{dom}(x) .
$$

Clearly this bound is weaker than the previous one, but well represents certain stability and performance specifications that emerge in reset control systems.

A further reason emphasizing the suitability of $t$-related bounds is that we will eventually augment reset control systems with so-called dwell-time, or temporal regularization constraints (see Chapter 4 and the subsequent ones) whose goal is to rule out solutions that stop flowing (such as the one represented at the right of Figure 2.3). With a dwell-time logic in place, it is possible to show that there exists $\rho>0$ such that all hybrid times $(t, j)$ in the domain of a solutions satisfy $t \geq \rho(j-1)$. Due to this fact, the hybrid exponential bound 2.5 and its $t$-restriction $(2.6)$ become equivalent. Following the paradigm behind transforming (2.5) into (2.6) we will discuss in this survey $t$-decay rates and $t$ - $\mathcal{L}_{2}$ gains for reset control systems with temporal regularization.

\subsection{An overview of this survey}

The tools introduced in this paper will illustrate the methods for proving the above exponential stability property for this specific example and also for some suitable generalizations of it. Below we discuss a point of view about the organization of the paper, stemming from certain considerations involving the elementary closed loop described in the previous section.

The survey is divided in three parts, the first one being an introductory part completed by this chapter. The second part deals with nonplanar reset systems and comprises four chapter covering several reset rules that may be used to augment high-order controllers for plants of any order. The second part of the book reports on a nontrivial generalization of the basic mechanisms emerging in Clegg integrators and First Order Reset Elements (FORE). We come back to those simpler ideas addressing a very simple class of SISO control systems with scalar plants and a scalar FORE in the third part of the survey. Despite the simplicity of such planar reset systems, interesting conclusions can be 
drawn and some experimental validations of the advantages arising from the use of reset controllers are also reported. We provide extra comments on the contents of each chapter in the remaining part of this section.

Chapter 3. The reset mechanism well represented in Figure 2.3 can be generalized to nonlinear continuous-time control systems with reset loops, is also the source for the design method later presented in Chapter 6. In particular, notice that a (Lyapunov-like) function $V(x)$ non increasing along flowing solutions of (2.4), corresponds to $V\left(x_{p}, x_{c}\right)=\left|\left[\begin{array}{c}x_{p} \\ x_{c}\end{array}\right]\right|^{2}$. For this function, one can check that the minimizer $\phi\left(x_{p}\right)=\operatorname{argmin}_{x_{c}} V\left(x_{p}, x_{c}\right)=0$, and it is possible to introduce the function $V_{p}\left(x_{p}\right):=V\left(x_{p}, \phi\left(x_{p}\right)\right)=\left|x_{p}\right|^{2}$, corresponding to the future value of $V\left(x^{+}\right)$if one decided to impose a jump of $x_{c}$ to $\phi\left(x_{p}\right)$. Then the selection of $\mathcal{F}$ and $\mathcal{J}$ is well understood in (2.4), as the sets from which (respectively) the function $V_{p}$ is nonincreasing and nondecreasing along solutions. A strict version of this reset mechanism is proposed in Chapter 6 .

Chapter 4 The illustration in Figure 2.3 of the possible solutions to (2.4) reveals that one has to expect solutions possibly performing an infinite number of jumps at the origin, and stopping to flow, thereby having a bounded domain in the $t$ direction (Zeno phenomenon). This fact may be problematic when wanting to prioritize flowing within a description of a continuous-time plant controlled by a reset controller. Moreover, it creates problems in higher order FORE control systems, where the origin of the $\left(e, x_{c}\right)$ plane may correspond to a whole subspace of the state space. Then Zeno solutions may emerge, which don't even converge to the origin. Due to this fact, and motivated by the need of using continuous-time Luenberger observers in the reset laws of the preceding chapter, we introduce and illustrate the concept of temporal regularization, or dwell-time mechanisms, in Chapter 4.

Chapter 5. Once the dwell-time mechanism has been introduced, all solutions will be forced to flow indefinitely (namely they have an unbounded domain in the $t$ direction) and are characterized by isolated jumps. This fact is a motivation for introducing suitable continuoustime gains and signal norms and take into account the effect of distur- 
bances acting on reset closed loops. For that scenario, Chapter 5 discusses ways to construct quadratic and nonquadratic Lyapunov functions that strictly decrease along solutions to (a temporally regularized version of) dynamics (2.4) and its higher order generalizations. In that context, the specific dynamics 2.4 constitutes an interesting example because it can be proven (see the discussion in Section 5.4 .3 for details) that no quadratic Lyapunov function exists that decreases along those solutions to 2.4 , whereas nonquadratic ones can be constructed and lead to interesting tight performance estimates.

Chapter 6. All of the material presented up to this point of the survey corresponds to stability and performance analysis tools. Chapter 6 combines the ideas of Chapters 3 and 5 for the design of a reset $\mathcal{H}_{\infty}$ plant-order controller for a linear plant.

Chapter 7. We conclude this overview paper by commenting on a somewhat parallel research direction coming back to the basic SISO reset mechanism described by (2.4) and its FORE generalizations stemming from (1.2), to derive tight necessary and sufficient stability conditions of planar reset closed loop, under a slightly modified dynamics that avoids the problem associated to the fact that solutions jumping from the horizontal line in the left plot of Figure 2.3 (this one belongs to $\mathcal{J}$ as one can inspect from the right of Figure 1.4) do not cause any change of $x$, and therefore of any possible Lyapunov function. Such a modification simplifies the analysis and leads to interesting conditions. Chapters 8 and 9 also discuss some interesting experimental results obtained using this FORE control system paradigm. 


\section{Part II}

\section{Non-planar reset systems}




\section{3}

\section{Nonlinear extensions of Clegg's integrator}

\subsection{A generalization of the FORE reset rule}

This part is inspired by Prieur et al. 2013] and exploits basic ideas on systems with mixed discrete/continuous dynamics, and Lyapunov theory for such hybrid dynamical systems. The basic idea consists in starting from an existing closed loop and to reset the controller as soon as the Lyapunov function is no more decreasing (or at least is decreasing sufficiently slowly with respect to the nominal decreasing of the Lyapunov function along the closed loop). This paradigm is useful not only for limiting the input updating of a control plant (when e.g. our approach is based on a modification of a given controller), but it may be also fruitful to update control inputs when the original closed loop is unstable. This latter case may even lead to better performance than considering only stabilized closed loop. Let us now proceed with more specific statement, and consider the following dynamical model for the plant:

$$
\dot{x}_{p}=f_{p}\left(x_{p}, u\right),
$$


with $x_{p}$ in $\mathbb{R}^{n_{p}}$, in feedback interconnection with a (not necessarily stabilizing) dynamic controller:

$$
\dot{x}_{c}=f_{c}\left(x_{c}, x_{p}\right), \quad u=h_{c}\left(x_{c}, x_{p}\right),
$$

with $x_{c}$ in $\mathbb{R}^{n_{c}}$. Then defining the closed-loop functions $\bar{f}_{p}\left(x_{p}, x_{c}\right)=$ $f_{p}\left(x_{p}, h_{c}\left(x_{c}, x_{p}\right)\right)$ and $\bar{f}_{c}\left(x_{c}, x_{p}\right)=f_{c}\left(x_{c}, x_{p}\right)$, the interconnection between (3.1) and (3.2) can be described in a compact way as:

$$
\dot{x}=f(x):=\left(\bar{f}_{p}\left(x_{p}, x_{c}\right), \bar{f}_{c}\left(x_{c}, x_{p}\right)\right),
$$

where $x:=\left(x_{p}, x_{c}\right), \bar{f}_{p}: \mathbb{R}^{n_{p}} \times \mathbb{R}^{n_{c}} \rightarrow \mathbb{R}^{n_{p}}$ and $\bar{f}_{c}: \mathbb{R}^{n_{c}} \times \mathbb{R}^{n_{p}} \rightarrow \mathbb{R}^{n_{c}}$. We assume that $f_{p}, f_{c}$ and $h_{c}$ are such that $\bar{f}_{p}$ and $\bar{f}_{c}$ are continuous functions satisfying $\bar{f}_{p}(0,0)=0$ and $\bar{f}_{c}(0,0)=0$.

The general objective of this chapter is to design a suitable reset rule, or jump law, for controller (3.2), and to design a partition of the state space $\mathbb{R}^{n}$ (where $n=n_{p}+n_{c}$ ) in two subsets, called flow and jump sets. The state $x_{c}$ of controller (3.2) endowed with such an additional logic, is then instantaneously reset according to the jump law whenever the state belongs to the jump set. This extended scheme, which is allowed to flow according to $(3.3)$ only if the state belongs to the flow set, defines a hybrid system. More specifically, the proposed hybrid augmentation is designed to guarantee the decrease of one or both of two Lyapunov-like functions: one of them $V: \mathbb{R}^{n_{p}} \times \mathbb{R}^{n_{c}} \rightarrow \mathbb{R}_{\geq 0}$, defined on the whole state space, and the other one $V_{p}: \mathbb{R}^{n_{p}} \rightarrow \mathbb{R}_{\geq 0}$ defined only in the plant state subspace. The functions $V$ and $V_{p}$ can be selected to capture some closed-loop stability and performance property (see the developments in Sections 3.5.1 and 3.5.2 where maximized decay rate and output overshoot reduction are tackled, respectively). Functions $V$ and $V_{p}$ are linked to each other by a function $\phi: \mathbb{R}^{n_{p}} \rightarrow \mathbb{R}^{n_{c}}$ such that for all $\left(x_{p}, x_{c}\right)$ in $\mathbb{R}^{n_{p}} \times \mathbb{R}^{n_{c}}$

$$
V\left(x_{p}, \phi\left(x_{p}\right)\right) \leq V\left(x_{p}, x_{c}\right),
$$

and, in particular, by the relation

$$
V_{p}\left(x_{p}\right)=V\left(x_{p}, \phi\left(x_{p}\right)\right), \forall x_{p} \in \mathbb{R}^{n_{p}} .
$$

Within the above scenario, we will design flow and jump sets and jump rules such that the arising hybrid systems guarantee non-increase of $V$ or $V_{p}$, or both of them in two relevant cases: 
$(V)$ (addressed in Section 3.2.1) where the function $V(\cdot, \cdot)$ is given and satisfies suitable conditions guaranteeing the existence ${ }^{1}$ of $\phi(\cdot)$ from which $V_{p}(\cdot)$ can be derived according to (3.5);

$\left(V_{p}\right)$ (addressed in Section 3.2.2 where $V_{p}(\cdot)$ and $\phi(\cdot)$ are given (their existence resembles an asymptotic controllability assumption), from which $V(\cdot, \cdot)$ satisfying (3.4) and (3.5) will be constructed.

Section 3.4 deals with the special case where system 3.3$)$ is linear. In this case, quadratic versions of $V$ and $V_{p}$ can be constructed under reasonably weak properties required for the closed-loop dynamics. The particularization to the linear case allows to strengthen the nonlinear results by exploiting the homogeneity property of hybrid systems acting on cones and obeying linear flow and jump rules. Finally, as a last contribution of this part, we will show how to design $V_{p}$ in item $\left(V_{p}\right)$ to augment linear continuous-time control systems with hybrid loops that reduce the overshoot of a scalar plant output. In comparison to previous works, the aim is to design hybrid strategies to guarantee some asymptotic stability property by enforcing that suitable Lyapunov-like functions are not increasing along the hybrid solutions.

The arising hybrid closed loop resembles the so-called impulsive systems, considered e.g. in Haddad et al. 2001. However the objectives of Haddad et al. 2001] and of the present part are different. Indeed an inverse optimal control involving a hybrid nonlinear-non-quadratic performance functional is developed in Haddad et al. 2001, whereas here we provide a design method of a hybrid loop (namely the jump map and the jump/flow sets) to ensure asymptotic stability and nonincrease of suitable scalar functions. Our results are also linked to the event-triggered control literature (see Anta and Tabuada [2010]) for stability analysis of networked control systems, where it is necessary to reduce the number of times when the state is measured by the controller and the actuators are updated. The most important difference between the results mentioned above and our contribution is that in those works the resetting value for the state is uniquely associated to

\footnotetext{
${ }^{1}$ Here, to keep the discussion simple, it is assumed that $V$ is continuously differentiable and that there exists $\phi\left(x_{p}\right) \in \underset{\operatorname{argmin}}{ } V\left(x_{p}, x_{c}\right)$, which implies 3.4 .
} 
the transmission of a measurement sample, whereas in our results it depends on the Lyapunov-like functions that should not increase along solutions.

\subsection{Hybrid stabilization with reset loops: nonlinear case}

\subsubsection{Constructing $V_{p}$ from $V$}

In this section we consider the closed-loop nonlinear system (3.3) and a function $V$ of the closed-loop state to address item $(V)$ defined Section 3.1. To this aim, we make the following assumption on the function $V$.

Assumption 3.1. The function $V: \mathbb{R}^{n} \rightarrow \mathbb{R}_{\geq 0}$ is continuously differentiable and such that there exists a continuous differentiable function $\phi: \mathbb{R}^{n_{p}} \rightarrow \mathbb{R}^{n_{c}}$ such that

$$
\phi\left(x_{p}\right) \in \operatorname{argmin}_{x_{c} \in \mathbb{R}^{n_{c}}} V\left(x_{p}, x_{c}\right) .
$$

Moreover, there exists a class $\mathcal{K}$ function $\alpha$ such that, for all $x_{p}$ in $\mathbb{R}^{n_{p}}$, $x_{p} \neq 0$,

$$
\left\langle\nabla_{p} V\left(x_{p}, \phi\left(x_{p}\right)\right), \bar{f}_{p}\left(x_{p}, \phi\left(x_{p}\right)\right)\right\rangle<-\alpha\left(V\left(x_{p}, \phi\left(x_{p}\right)\right)\right.
$$

where $\nabla_{p} V$ denotes the gradient of $V$ with respect to its first argument.

Remark 3.1. In Assumption 3.1 we do not impose that (3.3) is globally asymptotically stable, because (3.7) requires the function $V$ to be decreasing only in the subset of the state space defined by $\left(x_{p}, x_{c}\right)=\left(x_{p}, \phi\left(x_{p}\right)\right)$. Nevertheless, if system (3.3) is globally asymptotically stable, then there exist a function $V: \mathbb{R}^{n} \rightarrow \mathbb{R}_{\geq 0}$ and a class $\mathcal{K}$ function $\alpha$ such that $\langle\nabla V(x), f(x)\rangle<-\alpha(V(x))$ for all $x \neq 0$, $x=\left(x_{p}, x_{c}\right) \in \mathbb{R}^{n}$, which implies (3.7). Moreover note that in Assumption 3.1, it is not required that $\operatorname{argmin}_{x_{c} \in \mathbb{R}^{n_{c}}} V\left(x_{p}, x_{c}\right)$ is a single valued map, but only that a continuous differentiable selection of this map does exist. For example, with $V\left(x_{p}, x_{c}\right)=x_{p}^{4}+x_{c}^{4}-x_{p}^{2} x_{c}^{2}$, we have $\operatorname{argmin}_{x_{c} \in \mathbb{R}^{n_{c}}} V\left(x_{p}, x_{c}\right)=\left\{x_{p},-x_{p}\right\}$ which is not a singleton even though Assumption 3.1 can be satisfied, e.g., with $\phi\left(x_{p}\right)=x_{p}$. 
A natural way to stabilize the closed-loop system (3.3) is to flow when one (or both) of $V$ and $V_{p}$ is strictly decreasing and to reset the $x_{c}$-component of the state to the value $\phi\left(x_{p}\right)$ (where strict decrease is guaranteed by (3.7) ) when the function is not decreasing. This leads to the following hybrid system

$$
\left\{\begin{aligned}
\dot{x} & =f(x), & & x \in \hat{\mathcal{F}}, \\
x^{+} & =\left(x_{p}^{+}, x_{c}^{+}\right)=\left(x_{p}, \phi\left(x_{p}\right)\right), & & x \in \hat{\mathcal{J}},
\end{aligned}\right.
$$

where $\hat{\mathcal{F}} \subset \mathbb{R}^{n}$ and $\hat{\mathcal{J}} \subset \mathbb{R}^{n}$ are suitable closed subsets of the state space such that $\hat{\mathcal{F}} \cup \hat{\mathcal{J}}=\mathbb{R}^{n}$. In particular, $\hat{\mathcal{F}}$ and $\hat{\mathcal{J}}$ are defined by suitably combining the following two pairs of sets arising, respectively, from the knowledge of $V$ and $V_{p}$ :

$$
\begin{array}{r}
\mathcal{F}=\left\{x \in \mathbb{R}^{n},\langle\nabla V(x), f(x)\rangle \leq-\bar{\alpha}(V(x))\right\} \\
\mathcal{J}=\left\{x \in \mathbb{R}^{n},\langle\nabla V(x), f(x)\rangle \geq-\bar{\alpha}(V(x))\right\} \\
\overline{\mathcal{F}}=\left\{x \in \mathbb{R}^{n},\left\langle\nabla V_{p}\left(x_{p}\right), \bar{f}_{p}\left(x_{p}, x_{c}\right)\right\rangle \leq-\bar{\alpha}\left(V_{p}\left(x_{p}\right)\right)\right\} \\
\overline{\mathcal{J}}=\left\{x \in \mathbb{R}^{n},\left\langle\nabla V_{p}\left(x_{p}\right), \bar{f}_{p}\left(x_{p}, x_{c}\right)\right\rangle \geq-\bar{\alpha}\left(V_{p}\left(x_{p}\right)\right)\right\}
\end{array}
$$

where $\bar{\alpha}$ is any class $\mathcal{K}$ function such that $\bar{\alpha}(s) \leq \alpha(s)$ for all $s \geq 0$ (this will be denoted next by the shortcut notation $\bar{\alpha} \leq \alpha$ ).

Theorem 3.1. Consider the closed-loop system (3.3) and a function $V \in C^{1}$. Assume that there exist functions $\phi$ and $\alpha$ satisfying Assumption 3.1. Then for any class $\mathcal{K}$ function $\bar{\alpha}$ satisfying $\bar{\alpha} \leq \alpha$ the following holds.

1. If $V$ is positive definite and radially unbounded, then the hybrid system $3.8,3.9$ with $\hat{\mathcal{F}}=\mathcal{F}$ and $\hat{\mathcal{J}}=\mathcal{J}$ is globally asymptotically stable and $V$ is non-increasing along solutions.

2. If $V_{p}$, defined in (3.5), is positive definite and radially unbounded, then the hybrid system $3.8,3.10$ with $\hat{\mathcal{F}}=\overline{\mathcal{F}}$ and $\hat{\mathcal{J}}=\overline{\mathcal{J}}$ is such that the plant state $x_{p}$ converges to zero, and $V_{p}$ is nonincreasing along solutions.

3. If $V$ is positive definite and radially unbounded, then the hybrid system 3.8, 3.9, 3.10 with $\hat{\mathcal{F}}=\mathcal{F} \cap \overline{\mathcal{F}}$ and $\hat{\mathcal{J}}=\mathcal{J} \cup \overline{\mathcal{J}}$ is globally asymptotically stable and both $V$ and $V_{p}$ are nonincreasing along solutions. 
Remark 3.2. The three items of Theorem 3.1 address, respectively, the three goals of guaranteeing suitable stability properties together with non-increase of $V, V_{p}$ and both of them. In particular, the flow and jump sets of the hybrid system (3.8) are defined in Theorem 3.1 from the knowledge of the Lyapunov-like function $V$. Note also that the hybrid system 3.8$),(3.10)$ with $\hat{\mathcal{F}}=\overline{\mathcal{F}}$ and $\hat{\mathcal{J}}=\overline{\mathcal{J}}$ characterized at item 2 may be unstable. In other words, with this selection of the flow and jump sets, the plant state converges to the origin, although the state of the controller may diverge (which may complicate the implementation of the associate controller). In the linear case, this item will be strengthened under a detectability assumption (see Theorem 3.5 reported later in this chapter).

Remark 3.3. It can be checked that, due to the assumption on the regularity of $\bar{f}_{p}$ and $\bar{f}_{c}$, and on the regularity of $\phi$ as considered in Assumption 3.1. the hybrid system satisfies certain conditions (called hybrid basic conditions in Goebel and Teel [2006] and Prieur et al. [2007]). Then the hybrid system (3.8) inherits several good structural properties (such as existence of solutions and robustness properties). Moreover, instead of the assumption that the function $\alpha$ is of class $\mathcal{K}$ in Theorem 3.1, it may be assumed a LaSalle-type assumption. More precisely, using the LaSalle invariance property for hybrid systems (see e.g. Goebel et al., 2009, Theorem 23, page 64]), this assumption may be replaced by: for any solution $x$ of $(3.3)$, if $\langle\nabla V(x), f(x)\rangle \equiv 0$, then $x \equiv 0$.

Remark 3.4. If the closed-loop system (3.3) is globally asymptotically stable, then selecting $V$ and $\alpha$ satisfying Assumption 3.1. the sets in 3.9) correspond to $\mathcal{F}=\mathbb{R}^{n}$ and $\mathcal{J}=\emptyset$, so that the reset systems characterized in items 2 and 3 of Theorem 3.1 coincide. Therefore selecting the jump and flow sets as in (3.10) a non-increasing property for both $V$ and $V_{p}$, together with GAS is obtained. Note however that as long as Assumption 3.1 is satisfied, the flow set is never empty because (3.7) guarantees that after any jump the state belongs to the interior of the flow set. 


\subsubsection{Constructing $V$ from $V_{p}$}

In this section we consider the closed-loop nonlinear system 3.3 and a function $V_{p}$ of the plant state to address item $\left(V_{p}\right)$ defined in Section 3.1 . To this aim, we make the following assumption on the function $V_{p}$.

Assumption 3.2. The function $V_{p}: \mathbb{R}^{n_{p}} \rightarrow \mathbb{R}_{\geq 0}$ is continuously differentiable and radially unbounded and there exist a continuously differentiable function $\phi: \mathbb{R}^{n_{p}} \rightarrow \mathbb{R}^{n_{c}}$, and a class $\mathcal{K}$ function $\alpha$ such that, for all $x_{p}$ in $\mathbb{R}^{n_{p}}, x_{p} \neq 0$,

$$
\left\langle\nabla V_{p}\left(x_{p}\right), \bar{f}_{p}\left(x_{p}, \phi\left(x_{p}\right)\right)\right\rangle<-\alpha\left(V_{p}\left(x_{p}\right)\right) .
$$

Note that, when the $x_{p}$-equation of the closed-loop system (3.3) is affine with respect to $x_{c}$, this condition is related to the asymptotic controllability to the origin (Artstein 1983]. In this case, a control law $\phi$ can be computed from a Control Lyapunov Function $V_{p}$ and from the so-called universal formulas (see Freeman and Kokotović [1996], Lin and Sontag [1991).

Assumption 3.2 is sufficient to construct a function $V$ satisfying Assumption 3.1 so that the design strategy of the previous section can be employed. In particular, let $M$ be any symmetric positive semidefinite matrix ${ }^{2}$ in $\mathbb{R}^{n_{c} \times n_{c}}$ and define $V: \mathbb{R}^{n_{p}} \times \mathbb{R}^{n_{c}} \rightarrow \mathbb{R}_{\geq 0}$ for all $\left(x_{p}, x_{c}\right)$ in $\mathbb{R}^{n_{p}} \times \mathbb{R}^{n_{c}}$,

$$
V(x)=V_{p}\left(x_{p}\right)+\left(x_{c}-\phi\left(x_{p}\right)\right)^{\top} M\left(x_{c}-\phi\left(x_{p}\right)\right) .
$$

Note that $V$ is continuously differentiable, and radially unbounded. Moreover, if $\phi(0)=0$ and $M>0$, then it is a positive definite function because, for each $x_{p}$, it is the sum of two positive definite terms, the first one strictly positive when $x_{p} \neq 0$ and the second one strictly positive when $x_{p}=0$. The following theorem is a straightforward application of Theorem 3.1 in light of the $V_{p}$ and $\phi$ given in Assumption 3.2 and of the $V$ in 3.12 . A nonlinear example illustrating the potential behind the nonlinear design of Theorem 3.1 is given in Section 3.3 below (see also Prieur et al. [2010]).

\footnotetext{
${ }^{2}$ Note that the matrix $M$ may be a function of $x$. This extra degree of freedom could be used to perform convenient selections of $V$.
} 
Theorem 3.2. Consider the closed-loop system (3.3) and a function $V_{p} \in C^{1}$. Assume that there exist functions $\phi$ and $\alpha$ satisfying Assumption 3.2. Given any symmetric positive semidefinite matrix $M \in$ $\mathbb{R}^{n_{c} \times n_{c}}$, the corresponding function $V$ in $(3.12)$, and any class $\mathcal{K}$ function $\bar{\alpha}$ satisfying $\bar{\alpha} \leq \alpha$, the three items of Theorem 3.1 hold.

\subsection{A nonlinear control system stabilized by adding a hybrid loop}

Let us illustrate the use of Theorem 3.2 on a nonlinear system satisfying Assumption 3.2. To do that, after introducing the nonlinear system under consideration in this section, we will check Assumption 3.2, and by applying Theorem 3.2 , we will see on simulations that the second item of Theorem 3.1 applies. To simulate the hybrid systems we use the simulator presented in Goebel et al., 2009, pages 78-81].

Consider the following plant in $\mathbb{R}^{2}$

$$
\left\{\begin{array}{l}
\dot{x}_{1}=x_{2}+x_{1}^{2} \\
\dot{x}_{2}=u+x_{1}^{2}
\end{array}\right.
$$

where $\left(x_{1}, x_{2}\right)$ is the plant state and $u$ stands for the control variable in $\mathbb{R}$. By using a backstepping method (see e.g. Krstić et al. [1995]), the following controller $u: \mathbb{R}^{2} \rightarrow \mathbb{R}$ defined, for all $\left(x_{1}, x_{2}\right)$ in $\mathbb{R}^{2}$, by

$$
u\left(x_{1}, x_{2}\right)=-2 x_{1}-2 x_{2}-3 x_{1}^{2}-2 x_{1}\left(x_{2}+x_{1}^{2}\right)
$$

and the positive definite function $V_{p}: \mathbb{R}^{2} \rightarrow \mathbb{R}_{\geq 0}$ defined, for all $\left(x_{1}, x_{2}\right)$ in $\mathbb{R}^{2}$, by

$$
V_{p}\left(x_{1}, x_{2}\right)=\frac{1}{2} x_{1}^{2}+\frac{1}{2}\left(x_{2}+x_{1}+x_{1}^{2}\right)^{2}
$$

may be computed. Assumption 3.2 holds with $\alpha(s)=\frac{s}{2}$, for all $s \geq 0$. To check the attractivity property, let us consider the initial condition $\left(x_{1}(0,0), x_{2}(0,0)\right)=(10,10)$ and let us numerically compute the solution to system $(3.13)$ in closed loop with the controller (3.14). The time evolution of the $x_{1}$ and $x_{2}$-variables, and of the controller $u$ are depicted on Figure 3.1 .

Now let us consider the system $(3.13)$ in closed loop with the controller $u=x_{3}$ where $\dot{x}_{3}=x_{3}-x_{1}^{3}$. This nonlinear closed-loop system is 

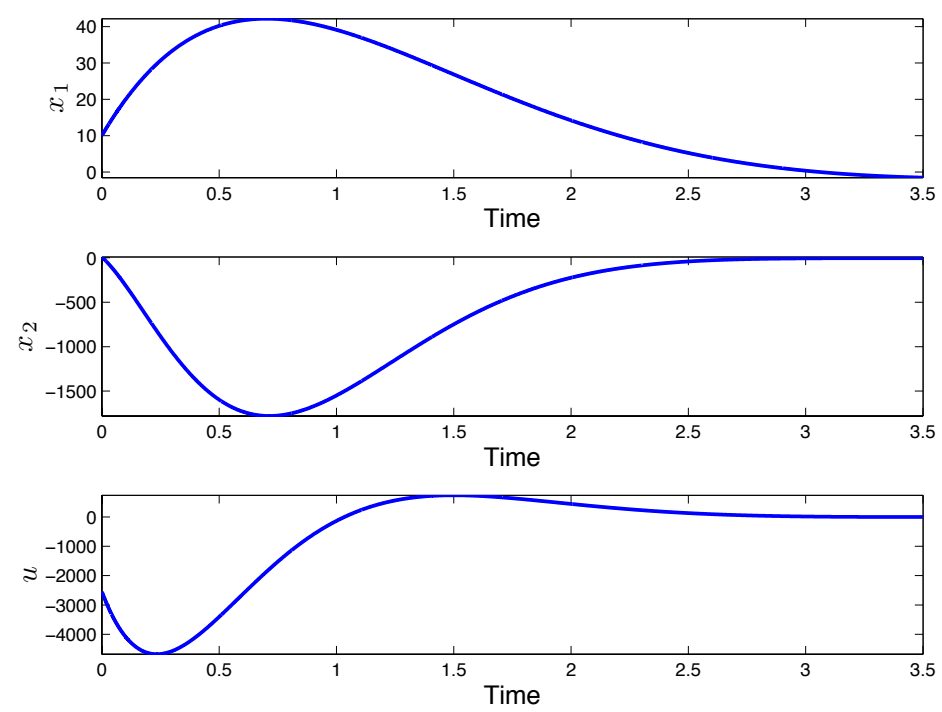

Figure 3.1: Time evolution of $x_{1}$ (top), of $x_{2}$ (middle) for system 3.13 in closedloop with the controller 3.14 (down).

unstable (the initial condition $\left(x_{1}(0,0), x_{2}(0,0), x_{3}(0,0)\right)=(10,10,10)$ gives a diverging solution). Let us apply Theorem 3.2 with $\bar{\alpha}=10^{-3} \alpha$. Consider the hybrid system $3.8,3.10$ with $\hat{\mathcal{F}}=\overline{\mathcal{F}}$ and $\hat{\mathcal{J}}=\overline{\mathcal{J}}$. The shape of the flow set of this hybrid system, intersected with the plane $x_{3}=0$ is given in Figure 3.2 (due to $(3.10)$, the jump set is the closure of the complement). Let us numerically compute the solution starting from the initial condition $\left(x_{1}(0,0), x_{2}(0,0), x_{3}(0,0)\right)=(10,10,10)$. We check on Figure 3.3 that the plant state $x_{p}$-variable is globally asymptotically stable. Moreover we note that the $x_{3}$-variable converges also to 0 and has some jumps (when it is reset to the value of the controller (3.14). The number of jumps depends on the size of $\mathcal{J}$ and thus on the choice of the function $\bar{\alpha}$. By comparing Figures 3.1 and 3.3 we note that the speed of convergence is improved using the hybrid system 3.8, 3.10 with $\hat{\mathcal{F}}=\overline{\mathcal{F}}$ and $\hat{\mathcal{J}}=\overline{\mathcal{J}}$.

The solutions issuing from different initial conditions are drawn on Figure 3.4 in the space $\left(x_{1}, x_{2}\right)$, and are projected in the space $\left(x_{1}, x_{3}\right)$ in Figure 3.5. The flow set is the convex hull of the set of the blue points 


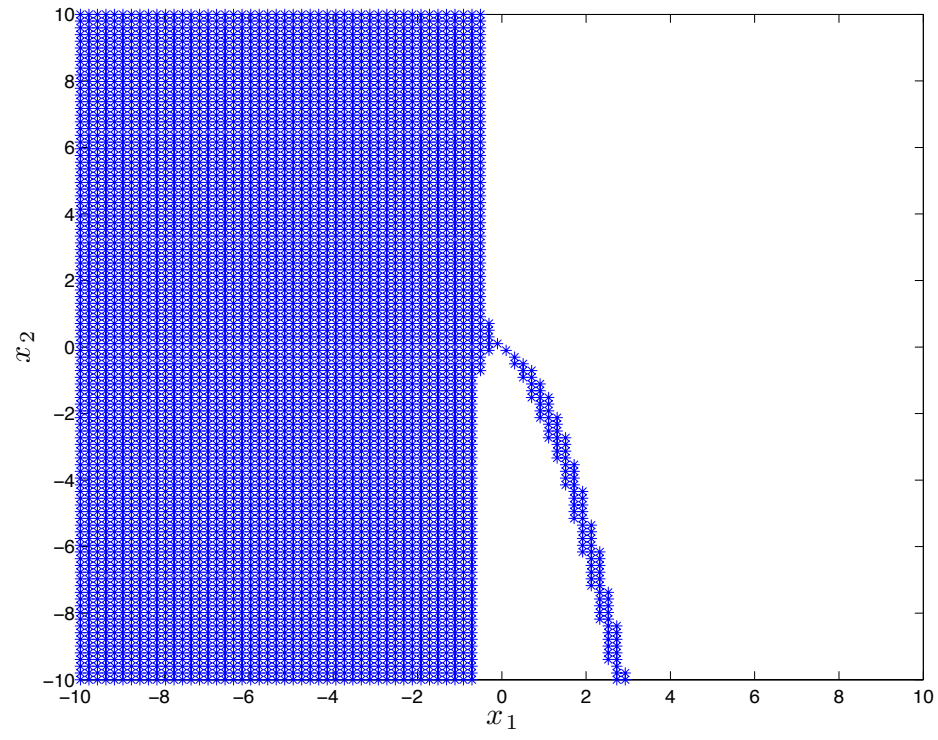

Figure 3.2: Shape of the flow set, intersected with the plane $x_{3}=0$, of the system 3.8, 3.10 with $\hat{\mathcal{F}}=\overline{\mathcal{F}}$ and $\hat{\mathcal{J}}=\overline{\mathcal{J}}$ in the box $[-10,10] \times[-10,10]$.

on Figure 3.4 The vertical lines are the jumps of the $x_{3}$-variable. It can be checked that they converge to the equilibrium (this is the center of the back ball on both figures).

\subsection{Hybrid stabilization with reset loops: linear case}

When focusing on linear dynamics, the two items $(V)$ and $\left(V_{p}\right)$ defined in Section 3.1 are addressed here. Consider the following linear plant:

$$
\dot{x}_{p}=A_{p} x_{p}+B_{p} u
$$

with $x_{p} \in \mathbb{R}^{n_{p}}$, in feedback interconnection with a (not necessarily stabilizing) linear dynamic controller:

$$
\dot{x}_{c}=A_{c} x_{c}+B_{c} x_{p}, \quad u=C_{c} x_{c}+D_{c} x_{p},
$$

with $x_{c} \in \mathbb{R}^{n_{c}}$. The closed loop is described by the following linear system

$$
\dot{x}=A x:=\left[\begin{array}{ll}
\bar{A}_{p} & \bar{B}_{p} \\
\bar{B}_{c} & \bar{A}_{c}
\end{array}\right] x,
$$



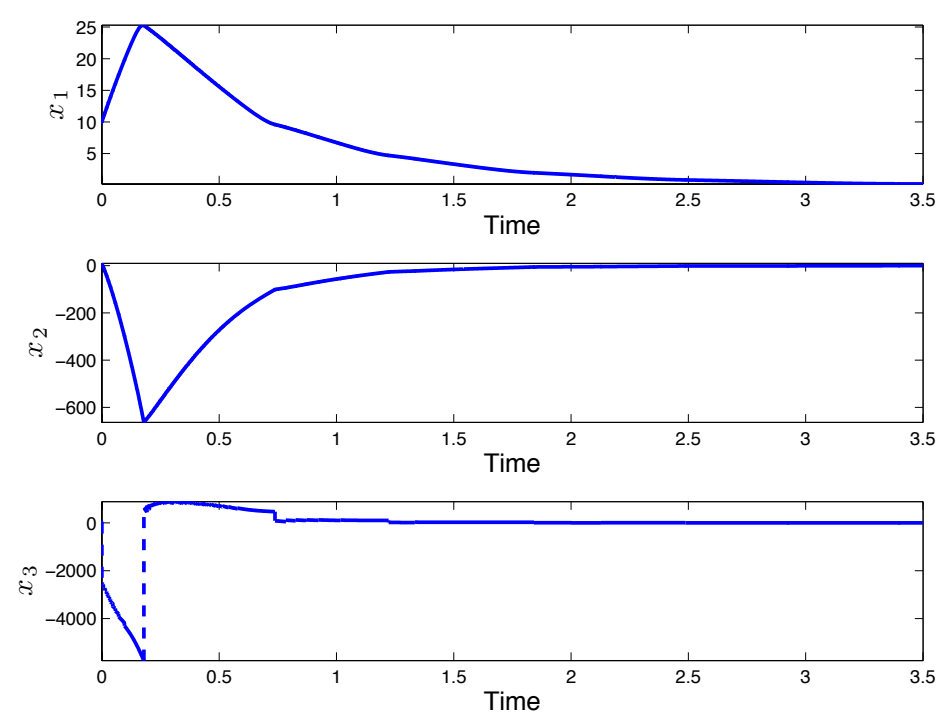

Figure 3.3: Time evolution of $x_{1}$ (top), of $x_{2}$ (middle) and of $x_{3}$ for flow time between 0 and 3.5 for the system $3.8,3.10$ with $\hat{\mathcal{F}}=\overline{\mathcal{F}}$ and $\hat{\mathcal{F}}=\overline{\mathcal{J}}$.

where $x=\left(x_{p}, x_{c}\right)$ and $\bar{A}_{p}, \bar{B}_{p}, \bar{A}_{c}$, and $\bar{B}_{c}$ are matrices of appropriate dimensions uniquely based on the matrices in (3.15) and (3.16). In the linear case, it is reasonable to restrict $V$ and $V_{p}$ to the class of quadratic functions, $\phi$ to the class of linear stabilizers and $\alpha$ and $\bar{\alpha}$ to the class of linear gains. Based on this, the closed-loop function $V$ can be selected as $V(x)=x^{\top} P x$ where $P=\left[\begin{array}{cc}P_{p} & P_{p c} \\ P_{p c}^{\top} & P_{c}\end{array}\right]$ is a symmetric positive definite matrix. Then, since $\nabla_{c} V\left(x_{p}, x_{c}\right)=2\left(P_{p c}^{\top} x_{p}+P_{c} x_{c}\right)$, from the positive definiteness of $P$, the critical points of $V$ are the points where $V$ is minimal. Thus the unique function $\phi: \mathbb{R}^{n_{p}} \rightarrow \mathbb{R}^{n_{c}}$ satisfying (3.4) is given, for all $x_{p} \in \mathbb{R}^{n_{p}}$, by

$$
\phi\left(x_{p}\right)=-P_{c}^{-1} P_{p c}^{\top} x_{p}=K_{p} x_{p} .
$$

Moreover, the function $V_{p}: \mathbb{R}^{n_{p}} \rightarrow \mathbb{R}_{\geq 0}$ defined by (3.5) becomes, for all $x_{p} \in \mathbb{R}^{n_{p}}$

$$
\begin{aligned}
V_{p}\left(x_{p}\right) & =\left[\begin{array}{c}
x_{p} \\
-P_{c}^{-1} P_{p c}^{\top} x_{p}
\end{array}\right]^{\top}\left[\begin{array}{cc}
P_{p} & P_{p c} \\
\star & P_{c}
\end{array}\right]\left[\begin{array}{c}
x_{p} \\
-P_{c}^{-1} P_{p c}^{\top} x_{p}
\end{array}\right] \\
& =x_{p}^{\top}\left(P_{p}-P_{p c} P_{c}^{-1} P_{p c}^{\top}\right) x_{p}=x_{p}^{\top} \bar{P}_{p} x_{p} .
\end{aligned}
$$




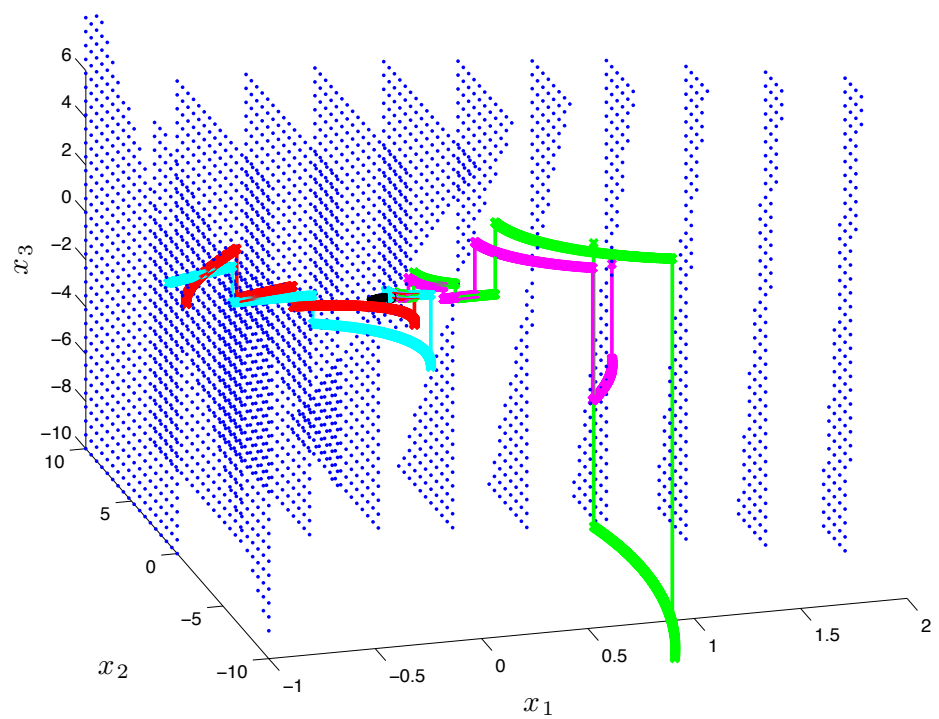

Figure 3.4: Shape of the flow set (in blue) and of some solutions (in green, red,...), for the system $3.8,3.10$ with $\hat{\mathcal{F}}=\overline{\mathcal{F}}$ and $\hat{\mathcal{J}}=\overline{\mathcal{J}}$. The equilibrium is the center of the back ball.

Based on (3.18) and (3.19), we get that (3.7) holds as long as there exists $\tilde{\alpha}>0$ such that

$$
\operatorname{He}\left(\bar{P}_{p}\left(\bar{A}_{p}+\bar{B}_{p} K_{p}\right)\right)<-\tilde{\alpha} \bar{P}_{p} .
$$

Thus, given $V$, if such a $\tilde{\alpha}>0$ exists, then Assumption 3.1 is guaranteed. Consider now the sets in 3.9 and 3.10 . Given any $0<\overline{\tilde{\alpha}} \leq \tilde{\alpha}$ and with the definitions above, after some calculations they become

$$
\begin{array}{r}
\mathcal{F}=\left\{x \in \mathbb{R}^{n}, x^{\top} N x \leq-\overline{\tilde{\alpha}} x^{\top} P x\right\}, \\
\mathcal{J}=\left\{x \in \mathbb{R}^{n}, x^{\top} N x \geq-\overline{\tilde{\alpha}} x^{\top} P x\right\}, \\
\overline{\mathcal{F}}=\left\{x \in \mathbb{R}^{n}, x^{\top} N_{p} x \leq-\overline{\tilde{\alpha}} x_{p}^{\top} \bar{P}_{p} x_{p}\right\}, \\
\overline{\mathcal{J}}=\left\{x \in \mathbb{R}^{n}, x^{\top} N_{p} x \geq-\overline{\tilde{\alpha}} x_{p}^{\top} \bar{P}_{p} x_{p}\right\},
\end{array}
$$

where ${ }^{3} N=\operatorname{He}\left[\begin{array}{ccc}P_{p} \bar{A}_{p}+P_{p c} \bar{B}_{c} & P_{p c} \bar{A}_{c}+P_{p} \bar{B}_{p} \\ P_{p c}^{\top} \bar{A}_{p}+P_{c} \bar{B}_{c} & P_{c} \bar{A}_{c}+P_{p c}^{\top} \bar{B}_{p}\end{array}\right]$ and $N_{p}=\operatorname{He}\left[\begin{array}{cc}\bar{P}_{p} \bar{A}_{p} \bar{P}_{p} \bar{B}_{p} \\ 0 \\ 0\end{array}\right]$.

\footnotetext{
${ }^{3} \mathrm{He}$ denotes the Hermitian matrix $\mathrm{He}(A)=A+A^{\top}$ for any matrix of $\mathbb{R}^{n \times n}$.
} 


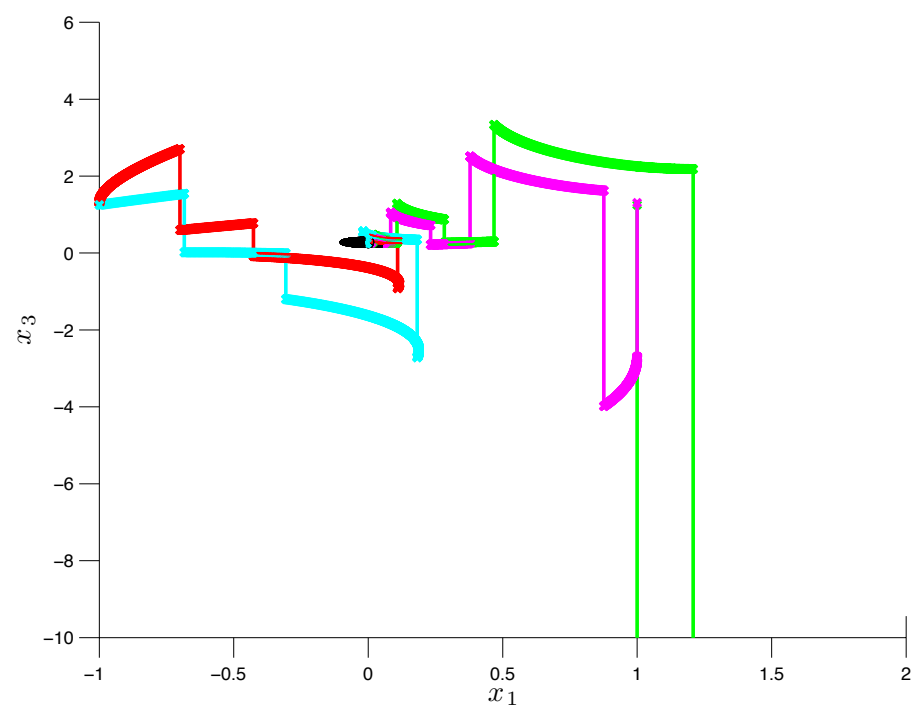

Figure 3.5: Projection to the $\left(x_{1}, x_{3}\right)$ plane of the shape of the flow set (in blue), of some solution (in green, red,...), for the system $3.8,3.10$ with $\hat{\mathcal{F}}=\overline{\mathcal{F}}$ and $\hat{\mathcal{J}}=\overline{\mathcal{J}}$. The equilibrium is the center of the back ball.

With the above definitions, the following corollary particularizes the results of Theorem 3.1 to the linear case.

Corollary 3.3. Consider the closed-loop system (3.17) and a function $V(x)=x^{\top} P x=x^{\top}\left[\begin{array}{cc}P_{p} & P_{p c} \\ P_{p c}^{\top} & P_{c}\end{array}\right] x$ such that $\bar{P}_{p}=P_{p}-P_{p c} P_{c}^{-1} P_{p c}^{\top}$ satisfies 3.20 for some $\tilde{\alpha}>0$ and for $K_{p}=-P_{c}^{-1} P_{p c}^{\top}$. Then the hybrid system

$$
\left\{\begin{aligned}
\dot{x} & =A x, & & x \in \hat{\mathcal{F}}, \\
x^{+} & =\left(x_{p}, x_{c}\right)^{+}=\left(x_{p}, K_{p} x_{p}\right), & & x \in \hat{\mathcal{J}},
\end{aligned}\right.
$$

satisfies all the items of Theorem 3.1 with $V_{p}\left(x_{p}\right)=x_{p}^{\top} \bar{P}_{p} x_{p}$ and using the sets in 3.21 with any $0<\overline{\tilde{\alpha}} \leq \widetilde{\alpha}$.

Remark 3.5. Let us apply Corollary 3.3 to linear plants in closed loop with reset controllers (see e.g. Beker et al. 2004], Nešić et al. [2008b]). Consider the linear plant $(3.15)$ with $A_{p}$ Hurwitz, $C_{c}=I_{n_{c}}$ and $D_{c}=0$. Recalling the notation in (3.17), when closing the loop with (3.16), we 
get $\bar{A}_{p}=A_{p}$ and $\bar{B}_{p}=B_{p}$, and thus $\bar{A}_{p}$ is Hurwitz. To represent reset controllers, let us consider $K_{p}=0$. Then there exist a symmetric positive definite matrix $\bar{P}_{p}$ in $\mathbb{R}^{n_{p} \times n_{p}}$ and $\tilde{\alpha}>0$ such that 3.20 holds. Applying Corollary 3.3 gives that for each symmetric positive definite matrix $P_{c}$ in $\mathbb{R}^{n_{c} \times n_{c}}$, the hybrid system

$$
\left\{\begin{aligned}
\dot{x} & =A x, & & x \in F_{l}, \\
x^{+} & =\left(x_{p}, x_{c}\right)^{+}=\left(x_{p}, 0\right), & & x \in J_{l},
\end{aligned}\right.
$$

where the flow and the jump sets are defined with $N_{l}=\operatorname{He}\left[\begin{array}{cc}P_{p} \bar{A}_{p} & P_{p} \bar{B}_{p} \\ P_{c} \bar{B}_{c} & P_{c} \bar{A}_{c}\end{array}\right]$, and for any $\overline{\tilde{\alpha}} \leq \tilde{\alpha}$, by

$$
\begin{gathered}
F_{l}=\left\{x \in \mathbb{R}^{n}, x^{\top} N_{l} x \leq-\overline{\tilde{\alpha}} x^{\top} P x\right\} \\
J_{l}=\left\{x \in \mathbb{R}^{n}, x^{\top} N_{l} x \geq-\overline{\tilde{\alpha}} x^{\top} P x\right\}
\end{gathered}
$$

is globally asymptotically stable and the function $V_{l}$ defined by $V_{l}(x)=$ $x^{\top} P x$, for any $x$ in $\mathbb{R}^{n}$, and for $P=\operatorname{diag}\left(P_{p}, P_{c}\right)$, is non-increasing. $\lrcorner$

Let us now specialize Theorem 3.2 to the linear case. With (3.17), Assumption 3.2 is satisfied whenever the pair $\left(\bar{A}_{p}, \bar{B}_{p}\right)$ is stabilizable and $V_{p}\left(x_{p}\right)=x_{p}^{\top} \bar{P}_{p} x_{p}$, with $\bar{P}_{p}>0$, is a Control Lyapunov Function for $\left(\bar{A}_{p}, \bar{B}_{p}\right)$. As a matter of fact in that case there exist a static state feedback matrix $K_{p}$ and a constant $\tilde{\alpha}>0$ such that equation 3.20 holds, and then equation (3.11) will hold with $\phi\left(x_{p}\right)=K_{p} x_{p}$. In particular, given $\bar{P}_{p}, K_{p}$ and $\tilde{\alpha}$ can be computed using a Linear Matrix Inequality (LMI) solver. Alternatively, under a stabilizability assumption, one can always solve a Generalized EigenValue Problem (GEVP) and find an optimal pair $\left(\bar{P}_{p}, K_{p}\right)$ maximizing $\tilde{\alpha}$. Based on $V_{p}$ and $K_{p}$, consider any symmetric positive definite matrix $P_{c}$ in $\mathbb{R}^{n_{c} \times n_{c}}$. The function $V: \mathbb{R}^{n_{p}} \times \mathbb{R}^{n_{c}} \rightarrow \mathbb{R}_{\geq 0}$ in $(3.12)$ can be defined as

$$
\begin{aligned}
V(x) & =V_{p}\left(x_{p}\right)+\left(x_{c}-K_{p} x_{p}\right)^{\top} P_{c}\left(x_{c}-K_{p} x_{p}\right) \\
& =x^{\top}\left[\begin{array}{c}
\bar{P}_{p}+K_{p}^{\top} P_{c} K_{p}-K_{p}^{\top} P_{c} \\
-P_{c} K_{p}
\end{array}\right] x=P_{c}^{\top} P x .
\end{aligned}
$$

Since $P_{c}>0$, this function is continuously differentiable, radially unbounded and positive definite. Then the following corollary of Theorem 3.2 can be stated. 
Corollary 3.4. Assume that the pair $\left(\bar{A}_{p}, \bar{B}_{p}\right)$ is stabilizable and that $V_{p}(x)=x_{p}^{\top} \bar{P}_{p} x_{p}$ is a control Lyapunov function for this pair. Then there exist $\tilde{\alpha}>0$ and $K_{p}$ satisfying (3.20). Moreover, given any symmetric positive definite matrix $P_{c}$ in $\mathbb{R}^{n_{c} \times n_{c}}$ and the corresponding function $V$ defined in (3.24), for any selection of $0<\overline{\tilde{\alpha}} \leq \tilde{\alpha}$, the reset system (3.22) with the sets in 3.21 satisfies all the items of Theorem 3.1

Remark 3.6. The global asymptotic stability (GAS) results established at items 1 and 3 of Theorem 3.1 can be strengthened to global exponential stability (GES) in the case of Corollaries 3.3 and 3.4 . Indeed, the reset system (3.21), 3.22 corresponds to a linear dynamics acting on conic flow and jump sets and, [Nešić et al., 2011, Theorem 7] can be applied to conclude that GAS implies GES.

As already pointed out in Remark 3.2 , when selecting $\hat{\mathcal{F}}=\overline{\mathcal{F}}$ and $\hat{\mathcal{J}}=\overline{\mathcal{J}}$, as in item 2 of Theorem 3.1, there is no guarantee that the state of the controller will converge to zero. This property is instead guaranteed when using $\hat{\mathcal{F}}=\overline{\mathcal{F}} \cap \mathcal{F}$ and $\hat{\mathcal{J}}=\overline{\mathcal{J}} \cup \mathcal{J}$, as in item 3 of Theorem 3.1. However, in light of Corollary 3.4, it would be desirable to provide conditions under which using the sets at item 2 of Theorem 3.1 is already sufficient to guarantee that the controller states converge to zero too. The advantage of this is that one would not need to bother with the selection of the matrix $P_{c}$ of Corollary 3.4 . because the sets $\overline{\mathcal{F}}$ and $\overline{\mathcal{J}}$ only depend on $V_{p}$ and $K_{p}$. In the linear case, it turns out to be sufficient that the controller dynamics is detectable from the output matrix $\bar{B}_{p}$. Intuitively, this requirement corresponds to asking that any nonzero controller evolution will be detected by the plant states so that a LaSalle result can be applied to show convergence.

Theorem 3.5. Consider any gain $K_{p}$ such that $\bar{A}_{p}+\bar{B}_{p} K_{p}$ is Hurwitz and a pair $\bar{P}_{p}, \tilde{\alpha}$ satisfying 3.20 . Consider any $0<\overline{\tilde{\alpha}} \leq \tilde{\alpha}$ and the flow and jump sets in $3.21 \mathrm{~b})$. If the pair $\left(\bar{B}_{p}, \bar{A}_{c}\right)$ is detectable, then the hybrid system at item 2 of Theorem 3.1 is globally exponentially stable. 


\subsection{Examples of linear systems involving hybrid loops}

In this section, we rely on the results of Theorem 3.5 to propose a suitable jump rule to be incorporated in a linear continuous-time control system to maximize the decay rate or reduce the plant output overshoot (see Sections 3.5.1 and 3.5.2, respectively).

\subsubsection{Maximizing the decay rate}

Consider equation (3.20) and note that it resembles the classical state-feedback stabilization problem with guaranteed convergence rate, which is well known to be solvable via a generalized eigenvalue problem. Given a plant, a possible way to address the search for $\bar{P}_{p}, K_{p}$ and $\tilde{\alpha}$ satisfying (3.20) is to impose a bound $\kappa_{M}$ on the size of $K_{p}$ and compute the maximum $\tilde{\alpha}$ satisfying (3.20) (or a conservative estimate of it) while guaranteeing $\left|K_{p}\right| \leq \kappa_{M}$. This type of goal is achieved by solving the following generalized eigenvalue problem:

$$
\begin{aligned}
\max _{\bar{Q}_{p}=\bar{Q}_{p}^{\top}, \tilde{\alpha}, X} & \tilde{\alpha}, \text { s.t. } \\
\bar{Q}_{p} & \geq I \\
0 & \leq\left[\begin{array}{cc}
\kappa_{M} I & X \\
X^{\top} & \kappa_{M} I
\end{array}\right] \\
-\tilde{\alpha} \bar{Q}_{p} & \geq \operatorname{He}\left(\bar{A}_{p} \bar{Q}_{p}+\bar{B}_{p} X\right),
\end{aligned}
$$

where $\kappa_{M}>0$ is given. The optimal solution to (3.25) leads to the gain $K_{p}=X \bar{Q}_{p}^{-1}$ and to $\bar{P}_{p}=\bar{Q}_{p}^{-1}$ satisfying 3.20 and such that $\left|K_{p}\right| \leq$ $|X|\left|Q_{p}^{-1}\right| \leq \kappa_{M}$ because the first constraint imposes $\left|Q_{p}^{-1}\right| \leq 1$ and the second constraint imposes $|X| \leq \kappa_{M}$. Using this gain, the following proposition holds (see, for example, Prieur et al. [2011], Fichera [2013] ) where it is provided a $t$-decay rate as introduced later in Definition 5.1 .

Proposition 3.1. Consider a symmetric positive matrix $\bar{Q}_{p}$ in $\mathbb{R}^{n_{p} \times n_{p}}$, a matrix $X$ in $\mathbb{R}^{n_{c} \times n_{p}}$ and a positive value $\tilde{\alpha}$ satisfying 3.25 . Let $K_{p}=X \bar{Q}_{p}^{-1}$ and $\bar{P}_{p}=\bar{Q}_{p}^{-1}$. For any selection of $0<\overline{\tilde{\alpha}} \leq \tilde{\alpha}$ and the flow and jump sets in (3.21b), then

1. for a suitable $k>0$, the plant state response satisfies the following 
exponential bound for all $(t, j) \in \operatorname{dom}(x)$ :

$$
\left|x_{p}(t, j)\right| \leq k \exp \left(-\frac{\overline{\tilde{\alpha}}}{2} t\right)\left|x_{p}(0,0)\right|
$$

2. if the pair $\left(\bar{B}_{p}, \bar{A}_{c}\right)$ is detectable, then the hybrid closed loop is globally exponentially stable.

The optimization problem in 3.25 can be used to build a curve providing the maximum decay $\tilde{\alpha}$ achievable with a certain bound $\kappa_{M}$ on the gain $K_{p}$. Then in many practical cases, it might well be that the optimal $\tilde{\alpha}$ grows unbounded as the bound $\kappa_{M}$ becomes arbitrarily large. This fact is illustrated in the next example.

Example 3.1. Consider a one-dimensional linear plant connected in negative feedback with a one-dimensional linear controller:

$$
\begin{aligned}
\dot{x}_{p} & =a_{p} x_{p}+b_{p} x_{c} \\
\dot{x}_{c} & =a_{c} x_{c}-x_{p},
\end{aligned}
$$

where we assume $b_{p}>0$.

For this example, the solution to (3.25) can be computed explicitly as a function of $\kappa_{M}$ and corresponds to $K_{p}=-\kappa_{M}$, while the optimal performance is $\tilde{\alpha}=-2 a_{p}+2 b_{p} \kappa_{M}$, achieved with $\bar{Q}_{p}=1$ and $X=$ $-\kappa_{M}$. It is instructive to study the shape of the jump and flow sets $\overline{\mathcal{F}}$ and $\overline{\mathcal{J}}$ defined in $3.21 \mathrm{~b}$, which are symmetric cones in the plane $\left(x_{p}, x_{c}\right)$. Using, for example, $\overline{\tilde{\alpha}}=\frac{\tilde{\alpha}}{2}=-a_{p}+b_{p} \kappa_{M}$, the sets in 3.21b become

$$
\mathcal{F}=\left\{\left(x_{p}, x_{c}\right):\left[\begin{array}{l}
x_{p} \\
x_{c}
\end{array}\right]^{\top}\left[\begin{array}{cc}
a_{p}+b_{p} \kappa_{M} & b_{p} \\
b_{p} & 0
\end{array}\right]\left[\begin{array}{l}
x_{p} \\
x_{c}
\end{array}\right] \leq 0\right\},
$$

while $\mathcal{J}$ is the closure of the complement of $\mathcal{F}$.

According to the results in Proposition 3.1 the hybrid closed-loop system given by

$$
\begin{cases}\dot{x}_{p}=a_{p} x_{p}+b_{p} x_{c}, & \\ \dot{x}_{c}=a_{c} x_{c}-x_{p}, & x \in \mathcal{F}, \\ x_{p}^{+}=x_{p}, & x \in \mathcal{J} \\ x_{c}^{+}=-\kappa_{M} x_{p}, & \end{cases}
$$



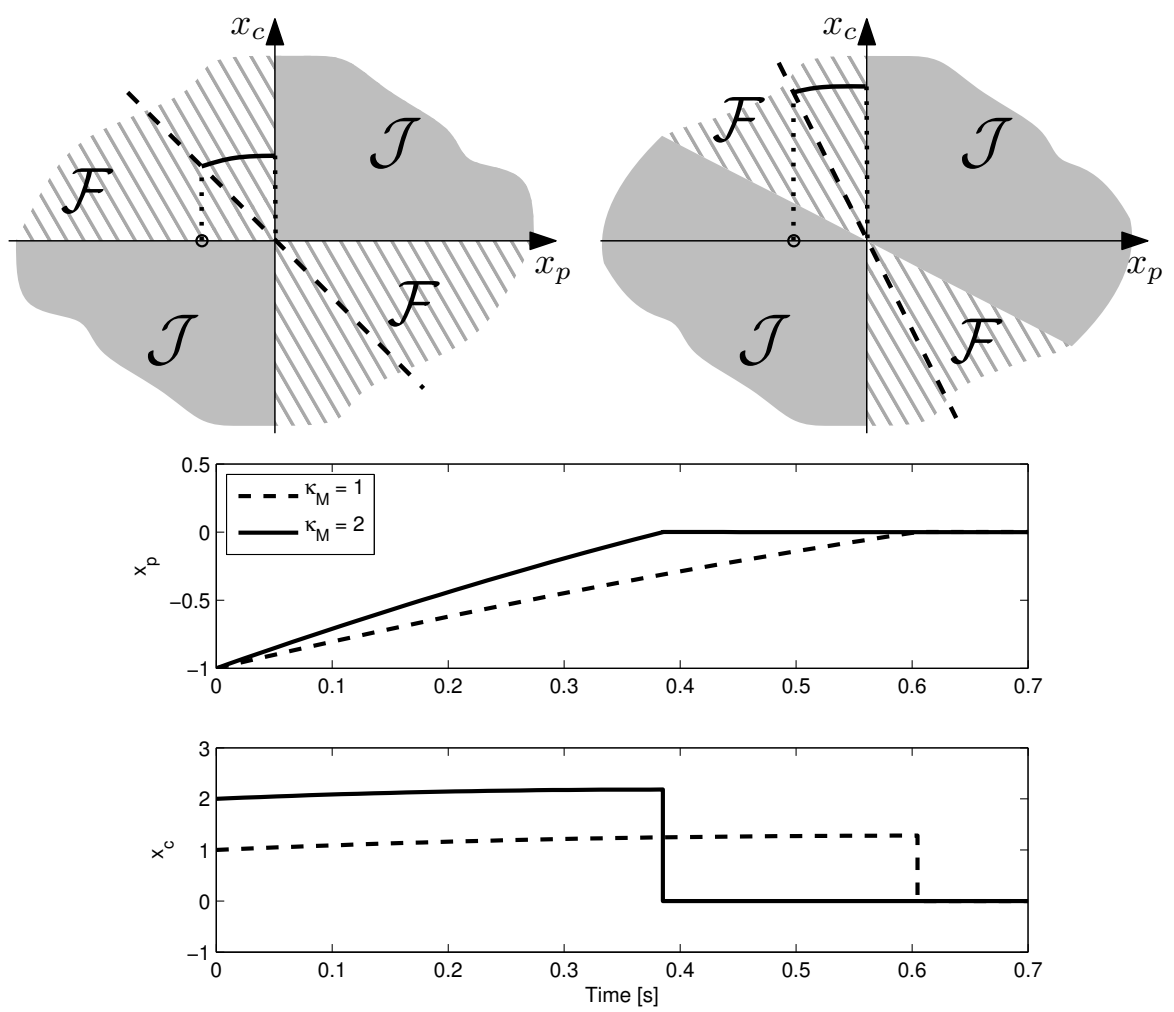

Figure 3.6: Shapes of the flow and jump sets of Example 3.1 using $\kappa_{M}=1$ (upper left) and $\kappa_{M}=2$ (upper right). The lower plots show the time histories of the two trajectories shown on the state space planes.

is globally exponentially stable.

The flow set in (3.26) defines a symmetric cone in the $\left(x_{p}, x_{c}\right)$ plane whose boundaries correspond to the two subspaces $\left\{\left(x_{p}, x_{c}\right): x_{p}=0\right\}$, namely the vertical axis, and $\left\{\left(x_{p}, x_{c}\right): 2 b_{p} x_{c}=-\left(a_{p}+b_{p} \kappa_{M}\right) x_{p}\right\}$, namely (by the fact that $b_{p}>0$ and $\kappa_{M}>0$ ), a line which rotates clockwise as $\kappa_{M}$ (and, consequently, the guaranteed convergence rate $\left.\overline{\tilde{\alpha}}=-a_{p}+b_{p} \kappa_{M}\right)$ increases. An example corresponding to $a_{p}=-1$, $b_{p}=1$ and $\kappa_{M}=1$ is shown in the top left plane of Figure 3.6. In this case, the flow set (striped area) corresponds to the second and fourth quadrants and the set $x_{c}=K_{p} x_{p}=-\kappa_{M} x_{p}=-x_{p}$, where the 
controller state is reset, (depicted as a bold dashed line) corresponds to the bisector of the second and fourth quadrant. The top right plane in the same figure shows how these sets change when increasing the gain to $\kappa_{M}=2$.

Some trajectories are also reported in Figure 3.6, corresponding to the case $a_{c}=0$ which, in the case $\kappa_{M}=1$ corresponds to a generalized version of the behavior of the so-called Clegg integrator (see Clegg [1958, Zaccarian et al. [2005]) in negative feedback interconnection with the plant $\mathcal{P}=\frac{1}{s+1}$. The generalization consists in resetting the Clegg integrator state to the value of the input rather than to zero but essentially coincides with the original resetting rule where the integrator was supposed to be reset only at zero crossings of the integrator input (see Clegg 1958, Zaccarian et al. 2005 for details). Note that in the case $\kappa_{M}=2$ reported to the right of Figure 3.6. the horizontal boundary of the flow set is tilted, reducing the size of the flow set, and the reset manifold $x_{c}=K_{p} x_{p}$ is tilted as well, to speed up the state decay rate. Nevertheless, the vertical boundary corresponding to $\left\{\left(x_{p}, x_{c}\right): x_{p}=0\right\}$ remains unchanged thus preserving the peculiarity that the response exhibits no overshoot. This property arises from the fact that the function $V_{p}\left(x_{p}\right)=x_{p}^{2}$ is non-increasing along the solutions. This fact is further illustrated by a few simulations starting from the initial conditions $\left(x_{p}(0,0), x_{c}(0,0)\right)=(-1,0)$, reported at the bottom plot of Figure 3.6. In both simulations, the controller state is first reset to the reset manifold and then exhibits a flow interval until the state reaches the vertical axis, where the solution is finally reset to zero.

\subsubsection{Overshoot reduction}

In this section, we rely on the results of Theorem 3.5 to propose a suitable jump rule to be incorporated in a linear continuous-time control system to reduce the plant output overshoot.

Perhaps the first example study where hybrid control systems were shown to overcome intrinsic limitations of classical linear control was the one in Beker et al. 2001a], where a reset controller was shown to improve upon linear control in terms of overshoot reduction. If the plant (3.15 is strictly proper (namely, $D_{p}=0$ ), a possible way to math- 
ematically formalize the requirement of overshoot reduction in terms of the Lyapunov functions introduced here is to construct a quadratic function $V_{p}\left(x_{p}\right):=x_{p}^{\top} \bar{P}_{p} x_{p}$ and the stabilizing gain $K_{p}$ in such a way that, to a certain extent, $V_{p}\left(x_{p}\right) \approx|y|^{2}$. Then achieving non-increase of $V_{p}$ via the hybrid loops proposed will induce (almost) no overshoot, namely $|y(t)|$ is (almost) non-increasing along trajectories.

Finding the above discussed pair $\left(V_{p}, K_{p}\right)$ can be done, for example, by writing the dynamics 3.15 in observability canonical form, so that $x_{p}=\left[\begin{array}{c}x_{1} \\ y\end{array}\right]$ and solving the following LMI eigenvalue problem ( $y$ is a single output):

$$
\begin{aligned}
\min _{\bar{Q}_{p}=\bar{Q}_{p}^{\top}, \rho_{x}, \rho_{y}, X} \rho_{y}, \text { s.t. } \bar{Q}_{p}=\left[\begin{array}{cc}
Q_{1} & q_{1 y} \\
q_{1 y}^{\top} & q_{y}
\end{array}\right]>I \\
0<\left[\begin{array}{cc}
\kappa_{M} I & X \\
X^{\top} & \kappa_{M} I
\end{array}\right], \quad 0>\operatorname{He}\left(\bar{A}_{p} \bar{Q}_{p}+\bar{B}_{p} X\right) \\
0 \leq\left[\begin{array}{cc}
\rho_{x} & 1 \\
1 & \rho_{y}
\end{array}\right], \quad \rho_{x} I<Q_{1}, \quad q_{y}<1+\rho_{y},
\end{aligned}
$$

where $\kappa_{M}>0$ is given. The optimal solution to (3.27) will lead to the gain $K_{p}=X \bar{Q}_{p}^{-1}$ and to $\bar{P}_{p}=\bar{Q}_{p}^{-1}$ satisfying (3.20) with a small enough $\tilde{\alpha}$ and such that $\left|K_{p}\right| \leq|X|\left|\bar{Q}_{p}^{-1}\right| \leq \kappa_{M}$. Moreover, the bounds given by the last three constraints should imply that smaller values of $\rho_{y}$ will lead to a function $V_{p}\left(x_{p}\right)=x_{p}^{\top} \bar{Q}_{p}^{-1} x_{p}$ closer to $|y|^{2}$.

Proposition 3.2. Consider a sequence of solutions $\left(\bar{Q}_{p}^{k}, \rho_{y}^{k}, \rho_{x}^{k}, X^{k}\right)_{k \in \mathbb{N}}$ to the optimization (3.27) such that $\rho_{y}^{k} \rightarrow 0$ as $k \rightarrow \infty$. Then, defining $V_{p}^{k}\left(x_{p}\right)=x_{p}^{\top}\left(\bar{Q}_{p}^{k}\right)^{-1} x_{p}$, we have for each $x_{p}, \lim _{k \rightarrow \infty} V_{p}^{k}\left(x_{p}\right)=|y|^{2}$, namely as $\rho_{y}^{k}$ approaches zero, $V_{p}^{k}\left(x_{p}\right)$ approaches $|y|^{2}$.

Moreover, given any pair $\left(\bar{Q}_{p}, X\right)$ satisfying 3.27$)$, let $K_{p}=X \bar{Q}_{p}^{-1}$ and $\bar{P}_{p}=\bar{Q}_{p}^{-1}$. Then for a small enough selection of $\tilde{\alpha}$, equation 3.20 is satisfied. Moreover, given any selection of $0<\overline{\tilde{\alpha}} \leq \tilde{\alpha}$, the reset system 3.22 with the flow and jump sets in $3.21 \mathrm{~b}$, is such that:

1. the plant state $x_{p}$ converges to zero, and the function $V_{p}\left(x_{p}\right)$ is non-increasing along solutions;

2. if the pair $\left(\bar{B}_{p}, \bar{A}_{c}\right)$ is detectable, then the hybrid closed loop is globally exponentially stable. 
From the point of view of the overshoot reduction, the meaning of the first statement of Proposition 3.2 is that smaller values of $\rho_{y}$ (which are expected to be achievable as the bound $\kappa_{M}$ on $\left|K_{p}\right|$ is increased) will guarantee that the function $V_{p}\left(x_{p}\right)$ becomes closer to the squared value $|y|^{2}$ of the output. One way to address overshoot elimination is to guarantee that the function $|y|^{2}$ itself is not increasing along solutions (indeed, as soon as $y(t)=0$ for some $t$, then $y$ will remain zero). Since our hybrid scheme ensures that $V_{p}$ does not increase along solutions, then the design in (3.27) leads to an "almost" non overshooting closed loop as long as $\rho_{y}$ is small enough. On the other hand, note that $\rho_{y}=0$ (namely, total overshoot elimination) cannot be achieved because we require that $V_{p}$ be positive definite in our theory.

Just as in the case discussed in Section 3.5.1, the optimization problem in 3.25 can be used to build a curve providing the overshoot reduction level $\rho_{y}^{-1}$ achievable with a certain bound $\kappa_{M}$ on the gain $K_{p}$. Similar to before, it might well be that the optimal $\rho_{y}^{-1}$ grows unbounded as the bound $\kappa_{M}$ becomes arbitrarily large.

Example 3.2. We consider an example originally discussed in Beker et al. 2001a, involving a FORE, whose flow dynamics follow the continuous-time transfer function $\frac{1}{s+1}$ and whose jump rule is to reset the state to zero whenever the input and the output of the FORE have opposite signs. This FORE is interconnected in negative unit feedback with a SISO plant whose transfer function is $\mathcal{P}=\frac{s+1}{s(s+0.2)}$. For this example, the control system involving the FORE is shown in Beker et al. 2001a to behave more desirably than the linear control system because it has only about $40 \%$ overshoot as compared to the linear closed-loop system, while retaining the rise time of the linear design (this example was also discussed later in Nešić et al. [2005], Zaccarian et al. 2011] where the $\mathcal{L}_{2}$ gain properties of the reset closed-loop were characterized). Here we show that when allowing more general resets than just the ones induced by the FORE resetting law (which essentially imposes a reset of the controller state to zero when the controller input and output have opposite signs), arbitrary small overshoot can be achieved, while retaining the same rise time. To this aim, we use the 
following state-space representation of the linear closed loop:

$$
\left[\begin{array}{c|c}
\bar{A}_{p} & \bar{B}_{p} \\
\hline \bar{B}_{c} & \bar{A}_{c}
\end{array}\right]=\left[\begin{array}{cc|c}
-0.6 & 0.6 & -1 \\
-0.4 & 0.4 & 1 \\
\hline 0 & 1 & -1
\end{array}\right] .
$$
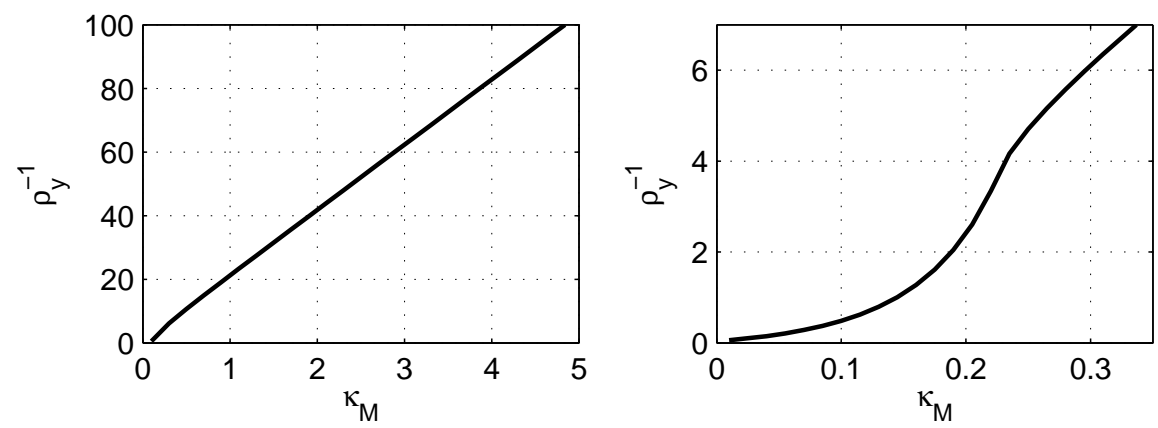

Figure 3.7: Curve of the overshoot reduction parameter $\rho_{y}^{-1}$ as a function of the bound $\kappa_{M}$ imposed on the gain $K_{p}$. The right plot is a zoom of the left one for small values of $\kappa_{M}$.

\begin{tabular}{|c|c|c|c|}
\hline$\kappa_{M}$ & $\bar{P}_{p}$ & $K_{p}^{\top}$ & $\rho_{y}^{-1}$ \\
\hline 0.1 & {$\left[\begin{array}{cc}0.31315 & -0.38064 \\
-0.38064 & 0.78906\end{array}\right]$} & {$\left[\begin{array}{c}0.046750 \\
-0.087302\end{array}\right]$} & 0.48451 \\
0.5 & {$\left[\begin{array}{cc}0.10170 & -0.088509 \\
-0.088509 & 0.09128\end{array}\right]$} & {$\left[\begin{array}{l}0.055896 \\
-0.49023\end{array}\right]$} & 10.662 \\
1 & {$\left[\begin{array}{cc}0.049336 & -0.045986 \\
-0.045986 & 0.99778\end{array}\right]$} & {$\left[\begin{array}{l}0.057423 \\
-0.97424\end{array}\right]$} & 21.179 \\
5 & {$\left[\begin{array}{cc}0.0097785 & -0.0096376 \\
-0.0096376 & 0.99991\end{array}\right]$} & {$\left[\begin{array}{l}0.059499 \\
-4.8307\end{array}\right]$} & 103.25 \\
\hline
\end{tabular}

Table 3.1: Values of the matrices $\bar{P}_{p}, K_{p}$ and $\rho_{y}^{-1}$ for some selections of $\kappa_{M}$.

Figure 3.7 shows the overshoot reduction parameter $\rho_{y}^{-1}$ as a function of the bound $\kappa_{M}$ imposed on the gain $K_{p}$. Note that the curve has a peculiar shape for small values of $\kappa_{M}$ and then essentially grows linearly for large values of $\kappa_{M}$. Table 3.1 reports the values of $\bar{P}_{p}, K_{p}$ and $\rho_{y}^{-1}$ for some selections of $\kappa_{M}$. Using the values in Table 3.1. we run several simulations of the closed-loop starting from the initial conditions $x_{p}(0,0)=\left[\begin{array}{ll}1 & 1\end{array}\right]^{\top}$, resembling the step responses reported in 
Beker et al. 2001a], Nešić et al. 2005], Zaccarian et al. 2011]. In particular, we implement the hybrid loops arising when using the values in the table within the scheme proposed in Proposition 3.2. Note that since in this case the pair $\left(\bar{B}_{p}, \bar{A}_{c}\right)$ is observable, then according to the results of Theorem 3.5 , the trajectories are all convergent to zero. The resulting plant input and output responses are shown in Figure 3.8 . where we also show the linear response (thin solid) and the response obtained with the FORE used in Beker et al. 2001a], Nešić et al. 2005, Zaccarian et al. 2011. (thin dashed).
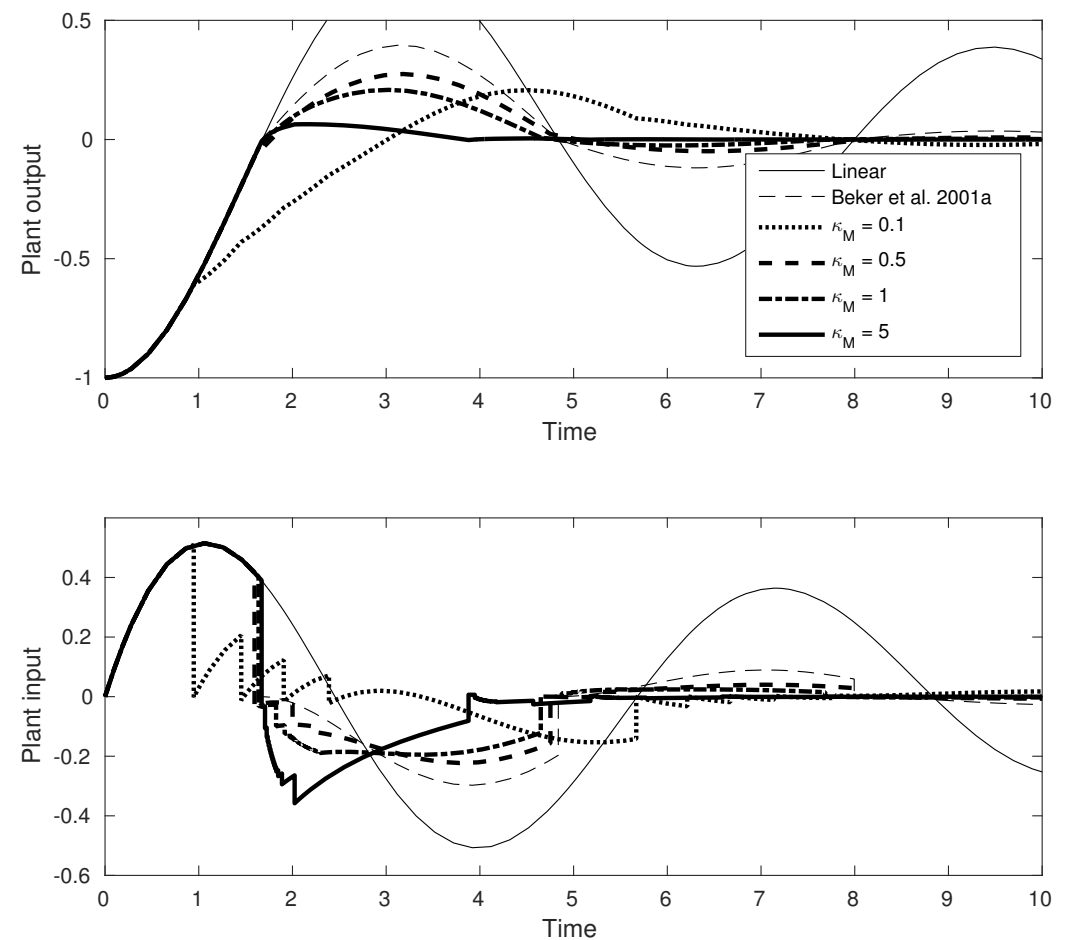

Figure 3.8: Simulation results when using the linear hybrid loops discussed in Example 3.2 Linear response (thin solid) and response of the FORE closed loop of Beker et al. 2001a. (thin dashed) compared to the reset responses obtained with the parameters in Table 3.1 (bold).

The results in Figure 3.8 are indicative of the potential of the proposed approach with the goal of reducing overshoots in linear control 
systems and correspond to trajectories of the reset system $(3.22)$ with the flow and jump sets in $3.21 \mathrm{~b})$, with the parameters in 3.28 and with the gains $K_{p}$ in Table 3.1. It should be pointed out that even though we manage to improve the overshoot reduction as compared to the FORE resetting strategy proposed in Beker et al. 2001a. (see the thin dashed curve in Figure 3.8), this last strategy is more appealing from an implementation viewpoint because it only requires a measurement of the plant output. Conversely, the resetting strategy of the improved bold curves of the figure are obtained using a full measurement of the plant state. 


\section{Dwell-time logic and observer-based hybrid loops}

\subsection{Overcoming two drawbacks of the hybrid loops sug- gested in Chapter 3}

The solution concept introduced in Chapter 2 well represents the fact that every time a solution jumps, there is some forward evolution (in the discrete-time direction) of the solution that is taken into account in its hybrid time domain. See for example the hybrid time domain represented at the left of Figure 2.1, where discrete time $j$ jumps from 0 to 1 at jump time $t_{1}$, then from 1 to 2 at jump time $t_{2}$, and similar for $t_{3}$.

A possible defective behavior arising in systems with jumps in the state (such as reset control systems) corresponds to the so-called "Zeno evolution" experienced by solutions that jump infinitely many times in a bounded time interval. Such a behavior characterizes a peculiar situation where the hybrid time domain is unbounded in the jump direction thereby remaining bounded in the flow direction. While this situation is well modeled mathematically in Goebel et al. 2012. (see also Goebel et al. 2009, page 72]), it is not reasonable to implement a control system that could generate solutions of this kind because unmodeled effects may cause the actual solution to exhibit an unpredictable behav- 


\subsection{Overcoming two drawbacks of the hybrid loops suggested in Chapter 3}

ior. From a practical viewpoint, the unsuitability of Zeno solutions for applications is also confirmed by the fact that simulations of systems that exhibit Zeno solutions cannot be continued after a finite ordinary time $t$.

The results presented in Chapter 3 propose hybrid loops that augment nonlinear continuous-time dynamic controllers for a continuoustime plant with the property of guaranteeing the non-increase of suitable Lyapunov-like functions, together with asymptotic stability of the origin. In particular, these hybrid loops can be understood as peculiar reset rules for the controller state that are triggered whenever the closed-loop state enters a specific region. Up to now, there are two main drawbacks of this approach: 1) the hybrid loops (namely the reset rules) require knowledge of the full state of the closed loop, to establish whether it belongs to the jump or to the flow set; 2) some solutions to the hybrid closed-loop system can experience a Zeno phenomenon, in particular when the solution approaches the equilibrium while being in the intersection of the flow and the jump sets (this is the case in Seuret et al. 2014 when the solution converges to the origin in finite time).

The goal of this chapter is to overcome these two drawbacks for linear plants when a hybrid state feedback law is given. To overcome the first drawback, a natural approach is to use an observer, to estimate the plant state, and to use this estimate when closing the hybrid loop. Such a separation principle only holds locally for nonlinear hybrid control systems (as established in Teel [2010]), however we succeed to propose in this chapter a scheme consisting in a hybrid output feedback controller embedding a Luenberger observer, which recovers asymptotically and globally the original scheme of the previous chapter as the observation error converges to zero.

The introduction of an observer in the scheme generates a number of problems arising from the fact that the Zeno solutions occurring at the origin become non-converging Zeno solutions in the presence of the observer. To avoid this defective phenomenon, paralleling the results in Forni et al. 2010, a control scheme has been proposed in Fichera et al. [2012a] where an arbitrarily small ball centered in the origin is removed from the jump set, so that, close to the origin, the observer 
is allowed to flow and to drive to zero the observation error. As a consequence, using the control scheme, the asymptotic stability results of the previous chapter become practical asymptotic stability results as it is proved that the solutions to the closed-loop system converge to this ball. Moreover, since convergence to zero of the plant state is not guaranteed anymore, Theorem 3.5 could not be recovered, thus providing a partial result for the output feedback case.

In this chapter a different control scheme is used, and we remove the defective Zeno solutions by augmenting the control system with a dwell-time logic which enforces a suitable dwell time between each pair of consecutive jumps (or resets). To do that, and following the strategy adopted in Nešić et al. 2005, Zaccarian et al. 2005 and later works such as Forni et al. [2014], reset control systems can then be augmented with an extra jump rule which imposes that after any jump a pre-assigned time interval $\rho$ has to expire before a subsequent jump is allowed. Each pair of jump times will then be not closer than $\rho$ to each other (namely, $\left|t_{i}-t_{j}\right|>\rho$ for all $i \neq j$ ), so that it will be impossible to have Zeno solutions. This type of rule has been used, e.g., in Johansson et al. [1999 and was therein called "temporal regularization". The same idea was also used in the context of reset systems in [Chen et al. 2001, §2]. The FORE (1.2) augmented with temporal regularization corresponds to the following second order hybrid system:

$$
\begin{aligned}
& \left\{\begin{array}{l}
\dot{\tau}=1-\mathrm{dz}\left(\frac{\tau}{\rho}\right), \\
\dot{x}_{c}=a_{c} x_{c}+b_{c} e,
\end{array} \quad \text { is allowed when } x_{c} e \geq 0 \text { or } \tau \in[0, \rho],\right. \\
& \left\{\begin{array}{l}
\tau^{+}=0, \\
x_{c}^{+}=0,
\end{array} \quad \text { is allowed when } x_{c} e \leq 0 \text { and } \tau \in[\rho, 2 \rho] .\right.
\end{aligned}
$$

where $\rho>0$ is the dwell time and $\operatorname{dz}(\cdot)$ is the standard unit deadzone.

The stability and performance results that we will report next all refer to reset control systems modified as in (4.1) where $\rho$ is a sufficiently small positive number. It should be emphasized that this temporal regularization of reset systems is mandatory even for just running a simulation. Indeed, simulation packages like Simulink will not be able to compute the solution forward in time if the temporal regularization is not implemented in the simulation scheme. As a consequence, temporal regularization is even more important if wanting to implement 


\subsection{Overcoming two drawbacks of the hybrid loops suggested in Chapter 3}

experimentally these reset control systems.

With the modified dynamics (4.1), it is convenient to take a closer look at the corresponding closed loop with a linear plant, generalizing dynamics (1.4). In particular, just as in Chapter 2, we may drop the sentence "is allowed when" from our equations and consider the general closed loop

$$
\begin{aligned}
& \left\{\begin{array}{l}
\dot{\tau}=1-\mathrm{dz}\left(\frac{\tau}{\rho}\right), \\
\dot{x}=A x+B d,
\end{array}\right. \\
& \left\{\begin{array}{l}
\tau^{+}=0, \\
x^{+}=G x,
\end{array} \quad x \in \mathcal{F} \text { or } \tau \in[0, \rho],\right.
\end{aligned}
$$

where $x \in \mathbb{R}^{n}, d \in \mathbb{R}^{m}$ and $\tau \in \mathbb{R}_{\geq 0}$.

If one selects $\mathcal{F}$ and $\mathcal{J}$ as the symmetric cones in 1.5 and $d$ is Lebesgue measurable, a solution $\xi(\cdot, \cdot):=(x(\cdot, \cdot), \tau(\cdot, \cdot))$ of the hybrid system 4.2 is a function defined on the hybrid time domain $\operatorname{dom}(\xi)=$ $\operatorname{dom}(x)=\operatorname{dom}(\tau)$ such that:

(i) for all $j$ and almost all $t$ such that $(t, j) \in \operatorname{dom}(\xi)$, we have

$$
(\xi(t, j) \in \mathcal{F} \text { or } \tau(t, j) \in[0, \rho]), \text { and }\left\{\begin{array}{l}
\dot{x}(t, j)=A x(t, j)+B d(t) \\
\dot{\tau}(t, j)=1-\mathrm{dz}\left(\frac{\tau(t, j)}{\rho}\right)
\end{array}\right.
$$

(ii) for all $(t, j) \in \operatorname{dom}(\xi)$ such that $(t, j+1) \in \operatorname{dom}(\xi)$ we have

$$
(\xi(t, j) \in \mathcal{J} \text { and } \tau(t, j) \in[\rho, 2 \rho]), \text { and }\left\{\begin{array}{l}
x(t, j+1)=G x(t, j) \\
\tau(t, j+1)=0
\end{array}\right.
$$

Due to the special structure of system 4.2 , and due to temporal regularization which enforces for all $(t, j) \in \operatorname{dom}(\xi), j \geq 1$ that $t_{j+1}-t_{j} \geq \rho$, Zeno solutions cannot occur and complete solutions (namely, solutions whose time domain is unbounded, or equivalently solutions that evolve forever) all have an unbounded domain in the $t$ direction (equivalently, given any complete solution $\xi(\cdot, \cdot)$, for any $t \in \mathbb{R}_{\geq 0}$, there exists $j \in \mathbb{Z}_{\geq 0}$ such that $(t, j) \in \operatorname{dom}(\xi))$. 


\subsection{Controller architecture and output feedback stabilization}

\subsubsection{Controller architecture}

According to the problem statement in Chapter 3 restricted to the linear case, we consider a linear time-invariant plant $\mathcal{P}$, represented by 3.15 with an output $y$, namely:

$$
\mathcal{P}\left\{\begin{aligned}
\dot{x}_{p} & =A_{p} x_{p}+B_{p} u \\
y & =C_{p} x_{p}+D_{p} u
\end{aligned}\right.
$$

with $x_{p} \in \mathbb{R}^{n_{p}}, u \in \mathbb{R}^{p}$ and $y \in \mathbb{R}^{q}$.

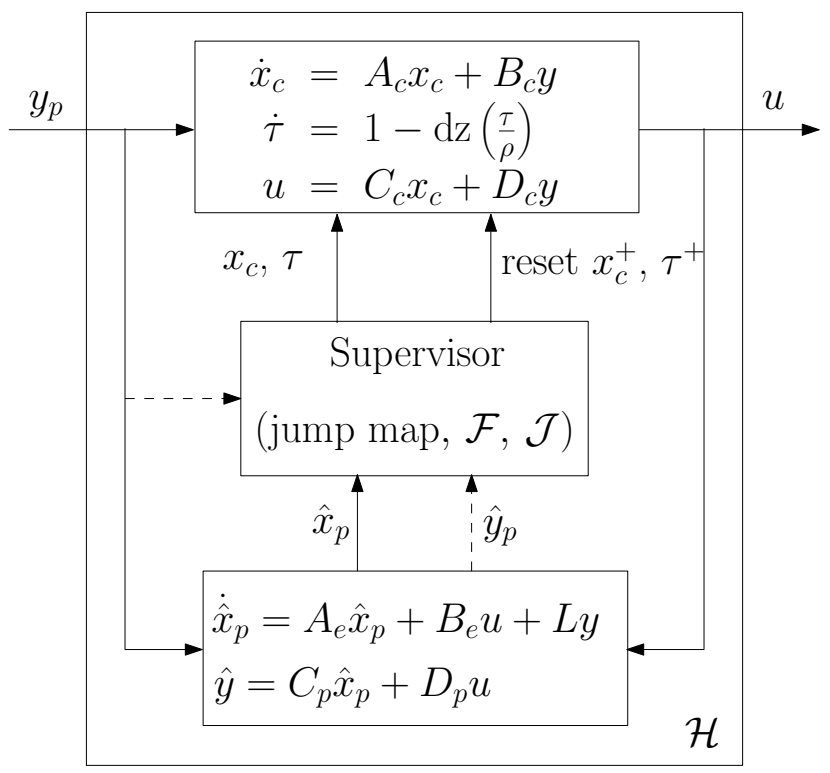

Figure 4.1: The proposed control scheme.

For the plant (4.3) we use the controller architecture shown in Figure 4.1, where the dynamic controller considered in Chapter 3, whose state is denoted by $x_{c}$, is augmented with a Luenberger observer, whose state is denoted by $\hat{x}_{p}$, with a dwell-time logic whose state is denoted by $\tau$ and with a supervisor enforcing the reset rules induced by the hybrid loops. In particular, while the linear closed loop between the dynamic controller and plant 4.3 does not make any use of the observed state, 
the observer state is used by the supervisor to decide when and where to reset the controller state.

In the next sections we will propose two solutions for the output feedback extension of the two architectures of Chapter 3 (thereby resulting in four schemes). All of the proposed schemes share the same flow dynamics which is

$$
\begin{aligned}
{\left[\begin{array}{c}
\dot{\hat{x}}_{p} \\
\dot{x}_{c}
\end{array}\right] } & =\left[\begin{array}{cc}
A_{e} & B_{e} C_{c} \\
0 & A_{c}
\end{array}\right]\left[\begin{array}{l}
\hat{x}_{p} \\
x_{c}
\end{array}\right]+\left[\begin{array}{c}
B_{e} D_{c}+L \\
B_{c}
\end{array}\right] y \\
& :=A_{\mathcal{H}}\left[\begin{array}{c}
\hat{x}_{p} \\
x_{c}
\end{array}\right]+B_{\mathcal{H}} y \\
u & =\left[\begin{array}{ll}
0 & C_{c}
\end{array}\right]\left[\begin{array}{l}
\hat{x}_{p} \\
x_{c}
\end{array}\right]+D_{c} y \\
& :=C_{\mathcal{H}}\left[\begin{array}{c}
\hat{x}_{p} \\
x_{c}
\end{array}\right]+D_{\mathcal{H}} y
\end{aligned}
$$

where $A_{e}=A_{p}-L C_{p}, B_{e}=B_{p}-L D_{p}$ and all the other matrices are design parameters that will be defined later. We assume that the Luenberger observer gain $L$ and the feedback interconnection of the plant-controller pair satisfy the following assumption.

Assumption 4.1. The interconnection (4.3)-(4.4) is well-posed, that is, the matrix $\left(I-D_{p} D_{c}\right)$ is non-singular. Moreover, the observer gain $L$ is such that the matrix $A_{e}=A_{p}-L C_{p}$ is Hurwitz.

Remark 4.1. Note that Assumption 4.1 implies that the pair $\left(C_{p}, A_{p}\right)$ is detectable.

To enforce a desirable dwell time between each pair of consecutive jumps of the hybrid control systems proposed next, we augment the state of the controller 4.4a), 4.4b with a timer (or dwell-time logic) $\tau \in \mathbb{R}$ (see also Cai et al., 2008, Proposition 1.1]). For the results in Teel et al. 2013 to be applicable, it will be important that this timer lies in a compact set. Therefore, instead of simply using $\dot{\tau}=1$ for our timer, we impose the following flow dynamics

$$
\dot{\tau}=1-\mathrm{dz}\left(\frac{\tau}{\rho}\right)
$$


From 4.4c), it is easy to see that the right-hand side is Lipschitz, that $\dot{\tau}=1$ if $\tau \in[0, \rho]$ and that the nonnegative interval $[0,2 \rho]$ is forward invariant. The timer $\tau$ will be restricted to the set $[0,2 \rho]$, by introducing reset to zero at each jump and by terminating any solution with $\tau \notin[0,2 \rho]$. Moreover, the timer will be used to inhibit jumps whenever $\tau \in[0, \rho)$.

For later use, after defining the observation error $e:=x_{p}-\hat{x}_{p}$ and the aggregated state $x:=\left[\hat{x}_{p}^{\top} x_{c}^{\top} e^{\top}\right]^{\top} \in \mathbb{R}^{n}$, with $n=2 n_{p}+n_{c}$, it is useful to rewrite the interconnection 4.3, 4.4 in the following compact form

$$
\begin{aligned}
{\left[\begin{array}{c}
\dot{\hat{x}}_{p} \\
\dot{x}_{c} \\
\dot{e}
\end{array}\right] } & =\left[\begin{array}{ccc}
\bar{A}_{p} & \bar{B}_{p} & B_{o} \\
\bar{B}_{c} & \bar{A}_{c} & \bar{B}_{c} \\
0 & 0 & A_{e}
\end{array}\right]\left[\begin{array}{c}
\hat{x}_{p} \\
x_{c} \\
e
\end{array}\right]:=A x \\
\dot{\tau} & =1-\mathrm{dz}\left(\frac{\tau}{\rho}\right) \\
y & =\left[\begin{array}{lll}
\bar{C}_{p} & \bar{C}_{c} & \bar{C}_{p}
\end{array}\right] x:=C_{y} x,
\end{aligned}
$$

with

$$
\begin{aligned}
{\left[\begin{array}{c|c|c}
\bar{A}_{p} & \bar{B}_{p} & B_{o} \\
\hline \bar{B}_{c} & \bar{A}_{c} & \bar{B}_{c} \\
\hline \bar{C}_{p} & \bar{C}_{c} & \bar{C}_{p}
\end{array}\right]=} & {\left[\begin{array}{c|c|c}
A_{p} & B_{p} C_{c} & L C_{p} \\
\hline 0 & A_{c} & 0 \\
\hline 0 & 0 & 0
\end{array}\right] } \\
& +\left[\begin{array}{c}
B_{p} D_{c} \\
\hline B_{c} \\
\hline I
\end{array}\right] X\left[C_{p}\left|D_{p} C_{c}\right| C_{p}\right],
\end{aligned}
$$

where $X=\left(I-D_{p} D_{c}\right)^{-1}$ is well defined and $A_{e}$ is Hurwitz from Assumption 4.1 .

Remark 4.2. From the cascaded structure of the closed-loop system 4.5a and the fact that $A_{e}$ is Hurwitz, it is evident that $\dot{e}=\dot{x}_{p}-\dot{\hat{x}}_{p}=$ $A_{e} e$, namely the observer state $\hat{x}_{p}$ converges exponentially to the plant state $x_{p}$, during flow.

\subsubsection{Reset rule replacing $x_{p}$ by $\hat{x}_{p}$}

The first state feedback reset rule proposed in Theorem 3.1 (see also Corollary 3.3 for the linear case addressed here) hinges upon the avail- 
ability of a Lyapunov-like function $\left(x_{p}, x_{c}\right) \mapsto V\left(x_{p}, x_{c}\right)$ which admits a sufficiently smooth minimizer $\phi\left(x_{p}\right)=\operatorname{argmin}_{x_{c}} V\left(x_{p}, x_{c}\right)$ satisfying

$$
V\left(x_{p}, \phi\left(x_{p}\right)\right) \leq V\left(x_{p}, x_{c}\right), \quad \forall x_{c} \in \mathbb{R}^{n_{c}} .
$$

In particular, when focusing on the linear case and using a quadratic Lyapunov function $V\left(x_{p}, x_{c}\right):=\left[\begin{array}{l}x_{p} \\ x_{c}\end{array}\right]^{\top} P\left[\begin{array}{l}x_{p} \\ x_{c}\end{array}\right]:=\left[\begin{array}{l}x_{p} \\ x_{c}\end{array}\right]^{\top}\left[\begin{array}{cc}P_{p} & P_{p c} \\ P_{p c}^{\top} & P_{c}\end{array}\right]\left[\begin{array}{l}x_{p} \\ x_{c}\end{array}\right]$, with $P=P^{\top}>0$, since the minimizer can be explicitly computed as $\phi\left(x_{p}\right):=-P_{c}^{-1} P_{p c}^{\top} x_{p}$, property 4.6 reduces to (see also 3.20 )

$$
\operatorname{He}\left(\bar{P}_{p}\left(\bar{A}_{p}+\bar{B}_{p} K_{p}\right)\right)<-\alpha \bar{P}_{p}, \quad \bar{P}_{p}=\bar{P}_{p}^{\top}>0,
$$

where

$$
\bar{P}_{p}:=P_{p}-P_{p c} P_{c}^{-1} P_{p c}^{\top}, \quad K_{p}:=-P_{c}^{-1} P_{p c}^{\top},
$$

and $\alpha \geq 0$ is a scalar design parameter. Note that by standard congruence transformations, constraint 4.7) on the entries of $P$ can be convexified (as done in Section 3.5.2).

To extend the corresponding construction to the output feedback case, we make the same assumption here.

Assumption 4.2. The plant $\mathcal{P}$ in $(4.3)$, and the matrix $P$ are such that equation (4.7), with the definitions in (4.8), is satisfied for some $\alpha>0$.

Using the definitions in 4.4, we select the hybrid controller of Figure 4.1 as

$$
\begin{aligned}
& \left\{\begin{array}{r}
{\left[\begin{array}{c}
\dot{x}_{p} \\
\dot{x}_{c}
\end{array}\right]=A_{\mathcal{H}}\left[\begin{array}{l}
\hat{x}_{p} \\
x_{c}
\end{array}\right]+B_{\mathcal{H}} y, \quad\left(\hat{x}_{p}, x_{c}\right) \in \mathcal{F} \text { or } \tau \in[0, \rho]} \\
\dot{\tau}=1-\mathrm{dz}\left(\frac{\tau}{\rho}\right), \\
\left\{\begin{array}{c}
\hat{x}_{p}^{+} \\
x_{c}^{+}
\end{array}\right]=\left[\begin{array}{cr}
I & 0 \\
\tau^{+} & =0
\end{array}\right]\left[\begin{array}{l}
\hat{x}_{p} \\
x_{c}
\end{array}\right], \quad\left(\hat{x}_{p}, x_{c}\right) \in \mathcal{J} \text { and } \tau \in[\rho, 2 \rho]
\end{array}\right. \\
& u=C_{\mathcal{H}}\left[\begin{array}{c}
\hat{x}_{p} \\
x_{c}
\end{array}\right]+D_{\mathcal{H}} y
\end{aligned}
$$


with the flow and jump sets chosen as:

$$
\begin{aligned}
& \mathcal{F}=\left\{\left[\begin{array}{l}
\hat{x}_{p} \\
x_{c}
\end{array}\right]:\left[\begin{array}{l}
\hat{x}_{p} \\
x_{c}
\end{array}\right]^{\top} N\left[\begin{array}{l}
\hat{x}_{p} \\
x_{c}
\end{array}\right] \leq-\tilde{\alpha}\left[\begin{array}{l}
\hat{x}_{p} \\
x_{c}
\end{array}\right]^{\top} P\left[\begin{array}{l}
\hat{x}_{p} \\
x_{c}
\end{array}\right]\right\}, \\
& \mathcal{J}=\left\{\left[\begin{array}{l}
\hat{x}_{p} \\
x_{c}
\end{array}\right]:\left[\begin{array}{c}
\hat{x}_{p} \\
x_{c}
\end{array}\right]^{\top} N\left[\begin{array}{l}
\hat{x}_{p} \\
x_{c}
\end{array}\right] \geq-\tilde{\alpha}\left[\begin{array}{c}
\hat{x}_{p} \\
x_{c}
\end{array}\right]^{\top} P\left[\begin{array}{l}
\hat{x}_{p} \\
x_{c}
\end{array}\right]\right\},
\end{aligned}
$$

where $0<\tilde{\alpha} \leq \alpha$ and

$$
N:=\operatorname{He}\left(P\left[\begin{array}{cc}
\bar{A}_{p} & \bar{B}_{p} \\
\bar{B}_{c} & \bar{A}_{c}
\end{array}\right]\right) .
$$

Using the compact representation 4.5, the interconnection (4.3) and (4.9) becomes the following hybrid closed-loop system:

$$
\begin{aligned}
& \left\{\begin{array}{l}
\dot{x}=A x, \\
\dot{\tau}=1-\mathrm{dz}\left(\frac{\tau}{\rho}\right),
\end{array}\right. \\
& \left\{\begin{array}{l}
x^{+}=G x, \\
\tau^{+}=0,
\end{array}\right. \\
& y=C_{y} x,
\end{aligned}
$$

where

$$
G:=\left[\begin{array}{ccc}
I & 0 & 0 \\
K_{p} & 0 & 0 \\
0 & 0 & I
\end{array}\right] .
$$

The closed-loop system 4.10, 4.11 generalizes to the output feedback case the state-feedback solution whose properties are established in Corollary 3.3 . The following theorem establishes the global exponential stability of the scheme.

Theorem 4.1. Consider a plant-controller pair (4.3), 4.9) satisfying Assumption 4.1 and two parameters $P, \alpha$ satisfying Assumption 4.2 . Then there exists $\rho^{*}>0$ such that for all $\rho \in\left(0, \rho^{*}\right]$, the set

$$
\mathcal{A}:=\{0\} \times[0,2 \rho] \subset \mathbb{R}^{n} \times \mathbb{R}
$$

is globally exponentially stable for the closed-loop system (4.11) with the sets $\mathcal{F}$ and $\mathcal{J}$ in 4.10. 
We focus now on the second state feedback reset rule proposed in Theorem 3.2 (see also Corollary 3.4 for the linear case addressed here). For this second case, the dynamics of the controller (4.11) unchanges but the jump and flow sets are defined based on a state feedback gain $K_{p} \in \mathbb{R}^{p \times n_{p}}$ for the plant (4.3) and a Lyapunov-like function which is only nonzero in the direction of the plant states, namely $V_{p}\left(x_{p}\right):=x_{p}^{\top} \bar{P}_{p} x_{p}$, where $\bar{P}_{p}$ is symmetric and positive definite. Also for this second case we make the following assumption, corresponding to Assumption 3.2 for the linear case (see also (3.6)).

Assumption 4.3. The plant $\mathcal{P}$ in 4.3$)$ and the matrix pair $\left(\bar{P}_{p}, K_{p}\right)$ are such that equation 4.7 is satisfied for some $\alpha>0$.

Based on the matrix pair $\left(\bar{P}_{p}, K_{p}\right)$ satisfying Assumption 4.3 and a (typically small) scalar $\epsilon>0$, we can now define the jump and flow sets associated with the second solution and to be used in the dynamics 4.11:

$$
\begin{aligned}
& \mathcal{F}=\left\{\left[\begin{array}{l}
\hat{x}_{p} \\
x_{c}
\end{array}\right]:\left[\begin{array}{l}
\hat{x}_{p} \\
x_{c}
\end{array}\right]^{\top} N_{p}\left[\begin{array}{l}
\hat{x}_{p} \\
x_{c}
\end{array}\right] \leq-\tilde{\alpha} \hat{x}_{p}^{\top} \bar{P}_{p} \hat{x}_{p}-\epsilon\left|x_{c}\right|^{2}\right\}, \\
& \mathcal{J}=\left\{\left[\begin{array}{l}
\hat{x}_{p} \\
x_{c}
\end{array}\right]:\left[\begin{array}{l}
\hat{x}_{p} \\
x_{c}
\end{array}\right]^{\top} N_{p}\left[\begin{array}{l}
\hat{x}_{p} \\
x_{c}
\end{array}\right] \geq-\tilde{\alpha} \hat{x}_{p}^{\top} \bar{P}_{p} \hat{x}_{p}-\epsilon\left|x_{c}\right|^{2}\right\},
\end{aligned}
$$

where $0<\tilde{\alpha} \leq \alpha$ and

$$
N_{p}:=\operatorname{He}\left(\left[\begin{array}{cc}
\bar{P}_{p} \bar{A}_{p} & \bar{P}_{p} \bar{B}_{p} \\
0 & 0
\end{array}\right]\right) .
$$

The closed-loop system (4.11), 4.13 generalizes to the output feedback case the state-feedback solution whose properties are established in Theorem 3.1 (see also Corollary 3.3. However, an extra term is added to allow inserting the dwell-time logic in the scheme without compromising the stability properties established in Chapter 3 . Indeed, while the flow and jump sets in 4.10) coincide with those of Corollary 3.3 , it is not true that the flow and jump sets in 4.13 coincide with those of Corollary 3.4 The difference stands in the term $-\epsilon\left|x_{c}\right|^{2}$ introduced here to provide a sufficient level of robustness. Such a robustness 
is required to tolerate the inevitable perturbations introduced by the dwell-time logic, which forces the system to flow even though $x$ belongs to $\mathcal{J}$ when the timer $\tau$ is too small. Note that the addition of this term does not restrict the class of systems that we consider, while it may lead to slightly smaller flow sets.

Although we do not have a formal proof of fragility of the scheme in Corollary 3.4, we should emphasize that the proofs of stability in Corollary 3.4 were based on the invariance principle because Lyapunov arguments only allowed to establish negative semidefiniteness of our candidate Lyapunov functions. It turns out that the term $-\epsilon\left|x_{c}\right|^{2}$ provides the missing decrease and significantly simplifies the proof of exponential stability of the state-feedback case here presented. Moreover the strict decrease arising from this term allows us to introduce the dwelltime logic without destroying the exponential stability of the closed loop. It should also be emphasized that the dwell-time parameter $\rho^{*}$ established in the next theorem shrinks to zero as $\epsilon$ becomes smaller. Perhaps, this suggests that the amount of the dwell-time perturbation, that the hybrid closed loop can tolerate, becomes smaller as $\epsilon$ in 4.13 shrinks to zero. To illustrate the effect of the new term $-\epsilon\left|x_{c}\right|^{2}$ in 4.13 , assume without loss of generality that $e=0$ (that is $x_{p}=\hat{x}_{p}$ ) and consider the following example

$$
A=\left[\begin{array}{cc}
\bar{A}_{p} & \bar{B}_{p} \\
\bar{B}_{c} & \bar{A}_{c}
\end{array}\right]=\left[\begin{array}{cc}
-1 & 0 \\
1 & 0
\end{array}\right], \quad \bar{P}_{p}=1,
$$

which, regardless of $K_{p}$ satisfies 4.7 for any $\alpha<1$ (because $\bar{B}_{p}=0$ ). This example does not satisfy the detectability condition in Theorem 3.5 and indeed one can see that setting $\epsilon=0$ in 4.13 (thus recovering the scheme of Theorem 3.5 the solutions starting from $\left(x_{p}, x_{c}\right)=$ $(0, a)$ for any $a \neq 0$ can flow indefinitely so that $x_{c}(t, j)=a \neq 0$ for all times, implying no convergence (even with dwell time).

Consider now the sets 4.13$)$ with $\epsilon \neq 0$ and $\left(x_{p}, x_{c}\right)=(0, a) \notin \mathcal{F}$ because $-\epsilon\left|x_{c}\right|^{2}=-\epsilon|a|^{2}<0$. Then $x_{c}$ is instantaneously forced to jump to $x_{c}^{+}=K_{p} x_{p}=0$, regardless of $K_{p}$, and this shows convergence. In other words, the extra term $-\epsilon\left|x_{c}\right|^{2}$ appearing in 4.13) (combined with the dwell-time logic) ensures that, upon convergence to zero of $x_{p}$, if $x_{c} \neq 0$, then the controller will eventually be forced to jump (as 
$x_{p}$ gets small enough) and the $x_{c}$ substate will be stabilized by way of the jumps without the need of the detectability of $\left(\bar{B}_{p}, \bar{A}_{c}\right)$ required in Theorem 3.5.

This last result is formalized in the next theorem which establishes global exponential stability of the scheme.

Theorem 4.2. Consider a plant-controller pair (4.3), (4.9) satisfying Assumption 4.1 and three parameters $\bar{P}_{p}, K_{p}$ and $\alpha$ satisfying Assumption 4.3. Then, for any $\epsilon>0$, there exists $\rho^{*}>0$ such that for all $\rho \in\left(0, \rho^{*}\right]$, the set $\mathcal{A}$ in $(4.12)$ is globally exponentially stable for the closed-loop system (4.11) with the sets $\mathcal{F}$ and $\mathcal{J}$ in 4.13.

\subsubsection{Enhanced reset rule exploiting $y-\hat{y}$}

The solution presented in the previous section succeeds in extending the schemes of Chapter 3 to the output feedback case, but it does not directly exploit the instantaneous knowledge of the output error $y-\hat{y}$ for the selection of the flow and jump sets and the reset rule. We explore this additional potential here and propose an enhanced scheme which is expected to behave better during the observer transient. The two enhanced schemes rely once again on the parameters $P, \alpha$ (respectively, the matrices $\bar{P}_{p}, K_{p}, \alpha$ ) satisfying Assumption 4.2 (respectively, Assumption 4.3), plus a set of four extra matrices: $K_{y} \in \mathbb{R}^{n_{c} \times q}$ used in the jump rule and $K_{x} \in \mathbb{R}^{n_{p} \times q}, K_{c} \in \mathbb{R}^{n_{c} \times q}, K_{\eta} \in \mathbb{R}^{q \times q}$ used in the flow and jump sets definition. The four matrices represent a set of additional free tuning parameters.

Let us define $\eta:=y-\hat{y}=C_{p}\left(x_{p}-\hat{x}_{p}\right)=C_{p} e$ and $\zeta:=\left[\hat{x}_{p}^{\top} x_{c}^{\top} \eta^{\top}\right]^{\top}$, the hybrid controller of Figure 4.1 is selected as:

$$
\begin{aligned}
& \left\{\begin{aligned}
{\left[\begin{array}{c}
\dot{\hat{x}}_{p} \\
\dot{x}_{c}
\end{array}\right] } & =A_{\mathcal{H}}\left[\begin{array}{l}
\hat{x}_{p} \\
x_{c}
\end{array}\right]+B_{\mathcal{H}} y \\
\dot{\tau} & =1-\mathrm{dz}\left(\frac{\tau}{\rho}\right)
\end{aligned}\right. \\
& \zeta \in \mathcal{F}_{y} \text { or } \tau \in[0, \rho] \\
& \left\{\begin{aligned}
{\left[\begin{array}{l}
\hat{x}_{p}^{+} \\
x_{c}^{+}
\end{array}\right] } & =\left[\begin{array}{cc}
I & 0 \\
K_{p} & 0
\end{array}\right]\left[\begin{array}{l}
\hat{x}_{p} \\
x_{c}
\end{array}\right]+\left[\begin{array}{c}
0 \\
K_{y}
\end{array}\right] \eta, \quad \zeta \in \mathcal{J}_{y} \text { and } \tau \in[\rho, 2 \rho] \\
\tau^{+} & =0,
\end{aligned}\right.
\end{aligned}
$$




$$
u=C_{\mathcal{H}}\left[\begin{array}{l}
\hat{x}_{p} \\
x_{c}
\end{array}\right]+D_{\mathcal{H}} y
$$

with the flow and jump sets selected as

$$
\begin{aligned}
& \mathcal{F}_{y}=\left\{\zeta:\left[\begin{array}{c}
\hat{x}_{p} \\
x_{c}
\end{array}\right]^{\top}(N+\tilde{\alpha} P)\left[\begin{array}{c}
\hat{x}_{p} \\
x_{c}
\end{array}\right] \leq\left[\begin{array}{c}
\hat{x}_{p} \\
x_{c} \\
\eta
\end{array}\right]^{\top}\left[\begin{array}{c}
K_{x} \\
K_{c} \\
K_{\eta}
\end{array}\right] \eta\right\}, \\
& \mathcal{J}_{y}=\left\{\zeta:\left[\begin{array}{c}
\hat{x}_{p} \\
x_{c}
\end{array}\right]^{\top}(N+\tilde{\alpha} P)\left[\begin{array}{c}
\hat{x}_{p} \\
x_{c}
\end{array}\right] \geq\left[\begin{array}{c}
\hat{x}_{p} \\
x_{c} \\
\eta
\end{array}\right]^{\top}\left[\begin{array}{c}
K_{x} \\
K_{c} \\
K_{\eta}
\end{array}\right] \eta\right\},
\end{aligned}
$$

where $0<\tilde{\alpha} \leq \alpha$ and $N$ and $P$ are selected according to the previous section. In 4.15 we use the notation $\mathcal{F}_{y}$ and $\mathcal{J}_{y}$ to insist on the fact that these sets depend on the output $y$ and controller state $x_{c}$ only, even if these sets are subsets of the full state space.

Using (4.5), the interconnection (4.3), 4.14) becomes the following hybrid closed-loop system:

$$
\begin{aligned}
& \left\{\begin{array}{l}
\dot{x}=A x, \\
\dot{\tau}=1-\mathrm{dz}\left(\frac{\tau}{\rho}\right),
\end{array}\right. \\
& \left\{\begin{array}{l}
x^{+}=G_{y} x, \\
\tau^{+}=0,
\end{array}\right. \\
& y=C_{y} x
\end{aligned}
$$

where

$$
G_{y}:=\left[\begin{array}{ccc}
I & 0 & 0 \\
K_{p} & 0 & K_{y} C_{p} \\
0 & 0 & I
\end{array}\right]
$$

Once again, the closed-loop system 4.15, 4.16 generalizes to the output feedback case the state-feedback solution whose properties are established in Corollary 3.3 . The following theorem establishes the global exponential stability of the scheme. 
Theorem 4.3. Consider a plant-controller pair 4.3), 4.14 satisfying Assumption 4.1. two parameters $P, \alpha$ satisfying Assumption 4.2 and four parameters $K_{y}, K_{x}, K_{c}, K_{\eta}$. Then there exists $\rho^{*}>0$ such that for all $\rho \in\left(0, \rho^{*}\right]$, the set $\mathcal{A}$ in 4.12 is globally exponentially stable for the closed-loop system 4.16 with the sets $\mathcal{F}_{y}$ and $\mathcal{J}_{y}$ in 4.15.

It should be emphasized that Theorem 4.3 only establishes the stability properties of the output feedback solution proposed here, whereas it does not highlight its strong relation with the parallel state-feedback solution of Chapter 3 . Such a relation is established in the next proposition. As in Goebel and Teel 2006, we say that two solutions are $(T, J, \varepsilon)$-close if their graphs are $\varepsilon$-close in the compact hybrid time domain bounded by $(T, J)$ (see Goebel and Teel [2006 for details). Moreover, we say that two solutions are $\varepsilon$-close if they are $(T, J, \varepsilon)$ close for all $(T, J) \in \mathbb{R}_{\geq 0} \times \mathbb{Z}_{\geq 0}$.

Proposition 4.1. Consider the state feedback hybrid closed loop in Corollary 3.3 . There exists $\rho^{*}>0$ such that for all $\rho \in\left(0, \rho^{*}\right]$ the following hold:

1. any solution of the state feedback hybrid closed loop starting from $\left(x_{p}(0,0), x_{c}(0,0)\right)=\left(x_{p 0}, x_{c 0}\right)$, with $x_{p 0} \neq 0$, is also the $\left(x_{p}, x_{c}\right)$ component of a solution of the output feedback hybrid closed-loop system 4.15), 4.16) (resp. 4.10), 4.11) starting from $\xi(0,0)=$ $\left(\hat{x}_{p}(0,0), x_{c}(0,0), e(0,0), \tau(0,0)\right)=\left(x_{p 0}, x_{c 0}, 0, \tau_{0}\right)$ with $\tau_{0} \geq \rho$;

2. for each $\epsilon>0$, there exists $\delta>0$ such that the $\left(x_{p}, x_{c}\right)$ component of any solution to the output feedback hybrid closedloop system (4.15), 4.16) (resp. 4.10, 4.11) starting from $\xi(0,0)=\left(\hat{x}_{p}(0,0), x_{c}(0,0), e(0,0), \tau(0,0)\right)=\left(x_{p 0}, x_{c 0}, e_{0}, \tau_{0}\right)$ with $x_{p 0} \neq 0, \tau_{0} \geq \rho$ and $\left|e_{0}\right| \leq \delta\left|\left(x_{p 0}, x_{c 0}\right)\right|$ is $\epsilon\left|\left(x_{p 0}, x_{c 0}\right)\right|$-close to a solution of the state feedback hybrid closed loop starting from $\left(x_{p}(0,0), x_{c}(0,0)\right)=\left(x_{p 0}, x_{c 0}\right)$.

Paralleling the previous section, we focus now on the second statefeedback reset rule proposed in Theorem 3.1 and we leave once again the dynamics of the controller (4.16) unchanged but we define different 
jump and flow sets, based on the pair of matrices $K_{p} \in \mathbb{R}^{p \times n_{p}}$ and $\bar{P}_{p} \in \mathbb{R}^{n_{p} \times n_{p}}, \bar{P}_{p}=\bar{P}_{p}^{\top}>0$, satisfying Assumption 4.3 . In particular, the jump and flow sets are defined as:

$\mathcal{F}_{y}=\left\{\zeta:\left[\begin{array}{l}\hat{x}_{p} \\ x_{c}\end{array}\right]^{\top}\left(N_{p}+\left[\begin{array}{cc}\tilde{\alpha} \bar{P}_{p} & 0 \\ 0 & \epsilon I\end{array}\right]\right)\left[\begin{array}{c}\hat{x}_{p} \\ x_{c}\end{array}\right] \leq\left[\begin{array}{c}\hat{x}_{p} \\ x_{c} \\ \eta\end{array}\right]^{\top}\left[\begin{array}{c}K_{x} \\ K_{c} \\ K_{\eta}\end{array}\right] \eta\right\}$,
$\mathcal{J}_{y}=\left\{\zeta:\left[\begin{array}{l}\hat{x}_{p} \\ x_{c}\end{array}\right]^{\top}\left(N_{p}+\left[\begin{array}{cc}\tilde{\alpha} \bar{P}_{p} & 0 \\ 0 & \epsilon I\end{array}\right]\right)\left[\begin{array}{c}\hat{x}_{p} \\ x_{c}\end{array}\right] \geq\left[\begin{array}{c}\hat{x}_{p} \\ x_{c} \\ \eta\end{array}\right]^{\top}\left[\begin{array}{c}K_{x} \\ K_{c} \\ K_{\eta}\end{array}\right] \eta\right\}$,

where $\epsilon>0,0<\tilde{\alpha} \leq \alpha$ and $N_{p}$ and $\bar{P}_{p}$ are selected in the same way as in the previous section.

The closed-loop system (4.16), 4.17) generalizes to the output feedback case the state-feedback solution whose properties are established in Theorem 3.1 (see also Corollary 3.3). The following theorem establishes the global exponential stability of the scheme.

Theorem 4.4. Consider a plant-controller pair (4.3), 4.14 satisfying Assumption 4.1. three parameters $\bar{P}_{p}, K_{p}$ and $\alpha$ satisfying Assumption 4.3 and five parameters $\epsilon>0, K_{y}, K_{x}, K_{c}, K_{\eta}$. Then there exists $\rho^{*}>0$ such that for all $\rho \in\left(0, \rho^{*}\right]$, the set $\mathcal{A}$ in $(4.12)$ is globally exponentially stable for the closed-loop system 4.16$)$ with the sets $\mathcal{F}_{y}$ and $\mathcal{J}_{y}$ in 4.17.

We cannot establish an equivalent statement to Proposition 4.1 with reference to the scheme (4.16), 4.17) and the linear state-feedback law of Corollary 3.4. Indeed, as emphasized at the end of Section 4.2.2, the jump and flow sets considered in Chapter 3 correspond to the ones in (4.17) with $e=0$ and $\epsilon=0$. Due to this fact, since we require $\epsilon>0$ here, we cannot say that the solutions to 4.16, 4.17) graphically converge to those of the corresponding state-feedback loops of Chapter 3. Nevertheless, since the system with a small $\epsilon>0$ corresponds to a perturbation of the system with $\epsilon=0$, we can state by relying on the 
results of Goebel and Teel 2006 that the arising trajectories can be made arbitrarily close to those of the state-feedback law of Corollary 3.4 by choosing $\epsilon$ arbitrarily small. Note that the same consideration may be done on Theorem 4.2 and solutions of 4.11, 4.13).

\subsection{Simulations}

In this section, two simulation examples are presented to show the effectiveness of the proposed methods. For the techniques in Theorems 4.1 and 4.3 , we use an example which appeared in several reset control papers. For the techniques in Theorems 4.2 and 4.4 , we present an example inspired by a positioning system comprising an electrical DC motor.

\subsubsection{Illustration of Theorems 4.1 and 4.3}

Consider the plant $P(s)=\frac{s+1}{s(s+0.2)}$ introduced in Beker et al. 2004 and discussed in Example 3.2 . According with (4.3), a possible realization is

$$
\left[\begin{array}{c|c}
A_{p} & B_{p} \\
\hline C_{p} & D_{p}
\end{array}\right]=\left[\begin{array}{cc|c}
-0.6 & 0.6 & -1 \\
-0.4 & 0.4 & 1 \\
\hline 0 & 1 & 0
\end{array}\right] .
$$

Notice that the pair $\left(C_{p}, A_{p}\right)$ is observable. To design the continuoustime part 4.4a of the controllers, we select the matrices $A_{c}, B_{c}, C_{c}$ and $D_{c}$ to define the same closed-loop system used in Beker et al. 2004 and in Example 3.2 , obtaining

$$
\left[\begin{array}{c|c}
A_{c} & B_{c} \\
\hline C_{c} & D_{c}
\end{array}\right]=\left[\begin{array}{c|c}
-1 & -1 \\
\hline 1 & 0
\end{array}\right] .
$$

For the hybrid part of our controller, we exploit the optimal configuration presented for the static state feedback in Example 3.2 for the overshoot reduction, see Table 4.1. As the basic idea of this optimization is to approximate the Lyapunov-like function, used to define the flow and jump sets, to the norm of the plant output (i.e. $\left.V\left(x_{p}, x_{c}\right) \approx|y|^{2}\right)$, we select $P_{c}=10^{-10}$ (namely the smallest $P_{c}$ that satisfies all the conditions in Assumption 4.2 in such a way that the influence of the 
state $x_{c}$ be reduced. Moreover we set $\tilde{\alpha}=10^{-8}$ to enlarge as much as possible the flow set and $\rho=2 \cdot 10^{-3}$. The observer gain in 4.4a is chosen as $L=\left[\begin{array}{ll}0.26 & 1.37\end{array}\right]^{\top}$ and the controller 4.16 is implemented with $K_{x}=0, K_{c}=0$, and $K_{\eta}=0$. As for $K_{y}$, the inspection of the transient response revealed that $K_{y}=-5$ leads to an improved transient, partially recovering the performance of the solid line obtained in Example 3.2 in which the knowledge of the state was assumed.

Figure 4.2 depicts the input and output behavior of the hybrid systems 4.11) (dashed line) and 4.16) (dot-dashed line) compared to the

Table 4.1: Hybrid controller setting proposed.

\begin{tabular}{|cc|c|c|}
\hline \multicolumn{2}{|c|}{$\bar{P}_{p}$} & $K_{p}^{\top}$ & $\alpha$ \\
\hline$\left[\begin{array}{cc}0.0097785 & -0.0096375 \\
-0.0096375 & 0.99990\end{array}\right]$ & {$\left[\begin{array}{c}0.0594992 \\
-4.83065\end{array}\right]$} & $7.784 \cdot 10^{-6}$ \\
\hline
\end{tabular}
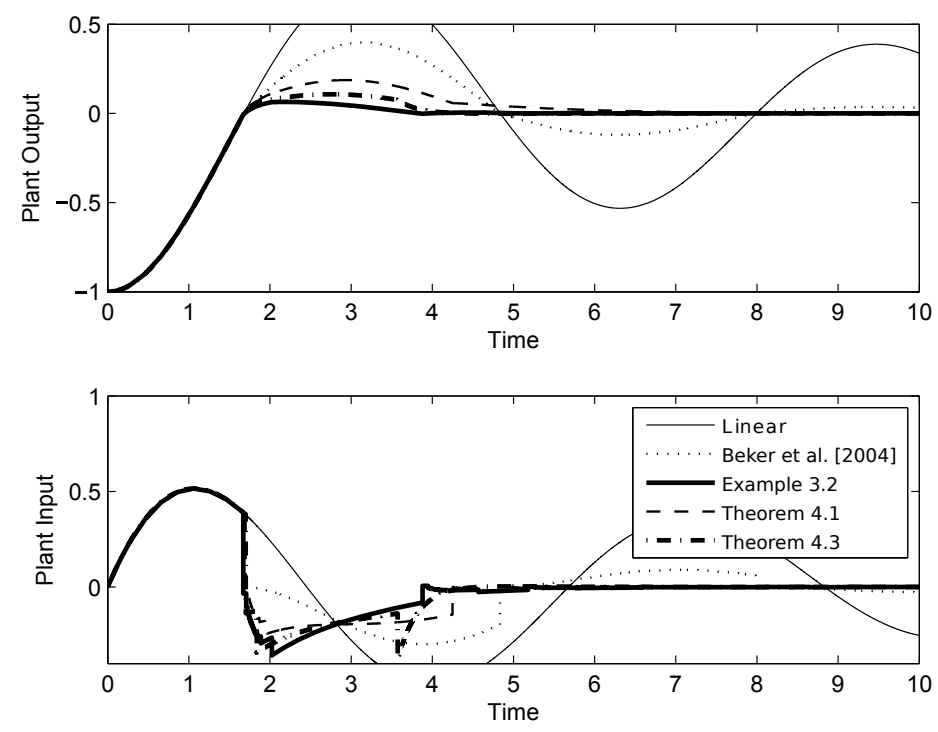

Figure 4.2: Hybrid controllers 4.9 and 4.14, compared to the linear case, to the FORE in Beker et al. 2004 and to the hybrid controller with optimal static state feedback given in Example 3.2 . 
linear case, to the technique in Beker et al. 2004 and to the technique in Example 3.2. All the controllers have zero initial conditions whereas the plant state starts from $x_{p}(0,0)=-\left[\begin{array}{ll}1 & 1\end{array}\right]^{\top}$. As expected, the undesired effects of the dynamics of the observer affect the controller 4.9 exhibiting a larger overshoot than the static state feedback of Example 3.2. This overshoot is caused by the observer transient. The second control technique is capable to partially compensate for this gap, recovering some performance for the output feedback case. We remark that, although the FORE in Beker et al. 2004 does not introduce further dynamics for the hybrid closed-loop system, it exhibits a larger overshoot than 4.11) and 4.16).

\subsubsection{Illustration of Theorems 4.2 and 4.4}

Let us consider the use of a DC motor already used in Fichera et al. 2012c to place a load in a desired position (namely, the origin). Figure 4.3 represents the series of the DC motor and the load that we are considering, where $K_{e}=10$ and $\tau_{e}=0.05$ are the electrical gain and time constant of the motor, $F=1.2$ and $J=0.5$ are the friction and inertia of the load. Note that $J=0.5$ corresponds, for example, to the inertia of a cylindric load with radius $R=0.25 \mathrm{~m}$ and mass $m=16 \mathrm{Kg}$.

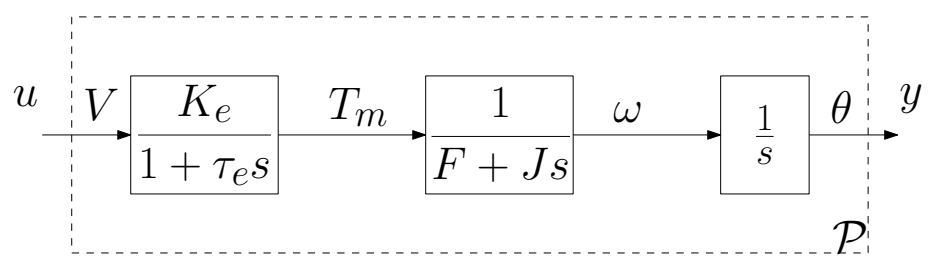

Figure 4.3: Plant block scheme.

With these parameters, the plant in observer canonical form is

$$
\left[\begin{array}{c|c}
A_{p} & B_{p} \\
\hline C_{p} & D_{p}
\end{array}\right]=\left[\begin{array}{ccc|c}
-22.4 & -6 & 0 & 50 \\
8 & 0 & 0 & 0 \\
0 & 1 & 0 & 0 \\
\hline 0 & 0 & 1 & 0
\end{array}\right],
$$


which can be commonly controlled by the following PI controller whose parameters have been tuned following a typical PI design procedure (see, e.g., Franklin et al., 2009, Chapter 4.3.4])

$$
\left[\begin{array}{c|c}
A_{c} & B_{c} \\
\hline C_{c} & D_{c}
\end{array}\right]=\left[\begin{array}{c|c}
0 & 0.1250 \\
\hline-0.08 & -0.05
\end{array}\right] .
$$

We want to augment this controller with a hybrid loop tuned in such a way to reduce the overshoot induced by the integral action. Two different hybrid loops are presented: one with a full order observer (namely, an observer with the same order as the plant) and one with a second order observer (designed based on a second order approximate model of the plant). In both cases, the optimization technique in Proposition 3.2 for the overshoot reduction is used. In all the plots, the linear PI controller will be compared to the results obtained with different settings of the same linear controller augmented by a hybrid loop.

Another aspect that will be taken into account is the robustness to parameters uncertainty. To this aim, we will consider the case in which the load has a mass $100 \%$ higher than expected, therefore the perturbed inertia value is $J=1$ (see Figure 4.3) and the perturbed plant matrices become

$$
\left[\begin{array}{c|c}
A_{p} & B_{p} \\
\hline C_{p} & D_{p}
\end{array}\right]=\left[\begin{array}{ccc|c}
-21.2 & -6 & 0 & 50 \\
4 & 0 & 0 & 0 \\
0 & 1 & 0 & 0 \\
\hline 0 & 0 & 1 & 0
\end{array}\right] .
$$

\section{Hybrid loop based on a full order observer}

Let us consider the technique in Proposition 3.2 , for $\kappa_{M}=0.1$ (with the same notation) and $L=\left[\begin{array}{llll}-0.0241 & 0.0841 & 1.0808\end{array}\right]^{\top}$, we get (according to (4.7) and (4.8)):

$$
\begin{aligned}
K_{p} & =\left[\begin{array}{lll}
0.00803345 & 0.02347159 & 0.09657843
\end{array}\right], \\
\rho_{y} & =0.2809 \\
\bar{P}_{p} & =\left[\begin{array}{lll}
0.02290376 & 0.06418020 & 0.06227308 \\
0.06418020 & 0.19173178 & 0.18092975 \\
0.06227308 & 0.18092975 & 0.95345625
\end{array}\right],
\end{aligned}
$$


where $\rho_{y}$ is the quantity to be minimized (according to the discussion after Proposition 3.2). Then, by selecting $\tilde{\alpha}=10^{-12}, \rho=0.004, \epsilon=$ 0.01 and $N_{p}$ as in $4.13 \mathrm{c}$, it is possible to define system 4.11 with the flow and jump sets in (4.13).

In all of the next simulations, the plant and controller initial conditions will be $x_{p}(0,0)=[0.70-4]^{\top}, x_{c}(0,0)=0$ and $\tau(0,0)=\rho$, whereas the observer initial condition is modified to explore the behavior of the hybrid controller. Choosing $\hat{x}_{p}(0,0)=x_{p}(0,0)$ (namely, the estimate error is zero), the output of the hybrid closed-loop system and the output of the linear closed loop with the simple PI are plotted in Figure 4.4(a) for the nominal case. Note that with a full order observer if $e(T)=0$ then $e(t)=0$ for all $t \geq T$ (in the absence of disturbances).

Figure 4.4(b) compares the behavior of the PI and the hybrid controllers with the plant subject to uncertainties of the parameters. The hybrid controller behavior is illustrated in several scenarios to better show how it is possible to compensate for the undesired effects caused by a non zero observation error. The overshoot reduction for the hybrid system is maintained in the case with parameters uncertainties when the estimate error of the observer is zero (dash-dotted line), but it may be lost if $e$ is large (dashed line). We remark that the faster rise time in the dashed line is not necessarily more desirable since it requires a larger control input (see lower plot). To compensate for this overshoot, we resort to the hybrid controller in 4.16 (same settings as for 4.11, plus a further term $K_{y}=0.06$ in the reset law) maintaining the flow and jump sets unchanged (namely, we select the sets in (4.17) with $K_{x}$, $K_{c}, K_{\eta}$ all zero). In this way we reduce the overshoot but the system still shows a faster rise time (dotted line). Finally, we introduce the gain $K_{\eta}=0.2$, which inhibits jumps when the output estimate error is large. The resulting response, corresponding to the thin solid line, appears to be closer to the state-feedback response.

\section{Hybrid loop based on an approximate reduced order observer}

Let us consider the plant of Figure 4.3 neglecting the first block, related to the electrical dynamics of the DC motor. Then the remaining part 

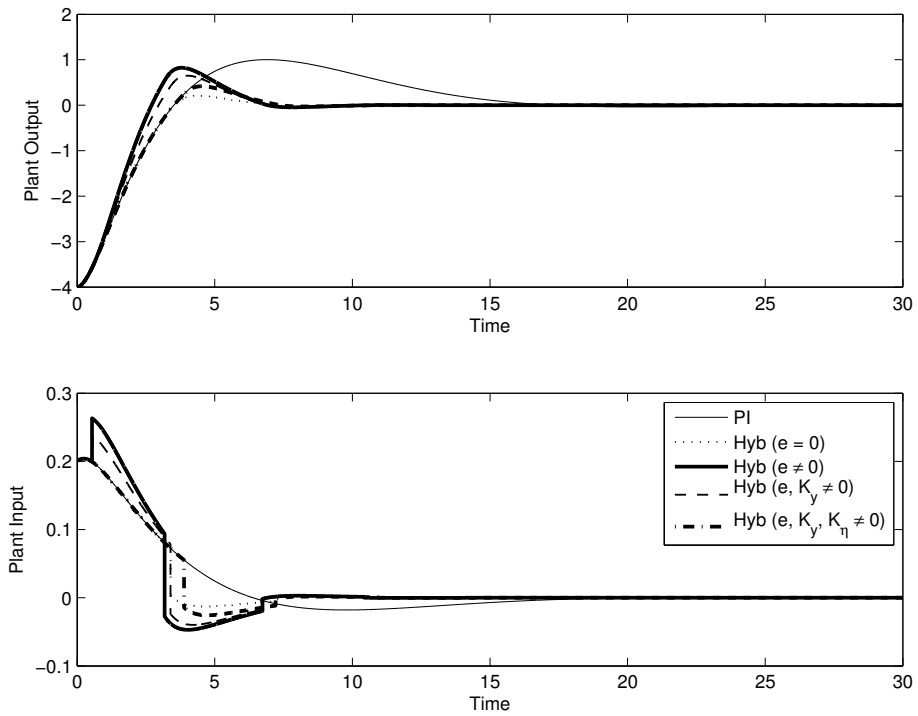

(a) Nominal case.
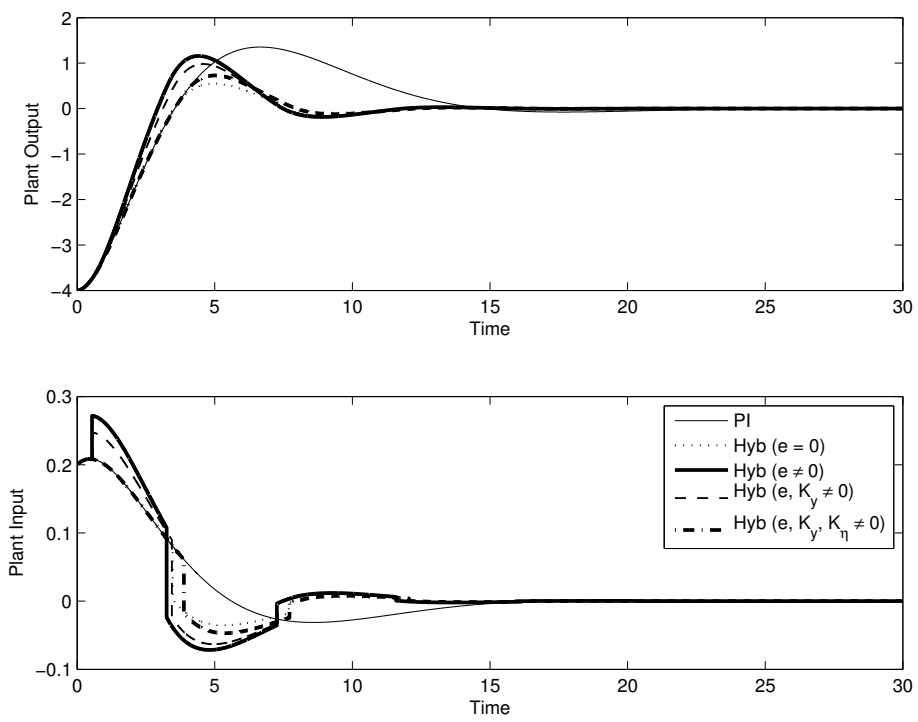

(b) Perturbed case.

Figure 4.4: Full order observer case (Example of Section 4.3.2). 
is

$$
\left[\begin{array}{c|c}
A_{p r} & B_{p r} \\
\hline C_{p r} & D_{p r}
\end{array}\right]=\left[\begin{array}{cc|c}
-2.4 & 0 & 2 \\
1 & 0 & 0 \\
\hline 0 & 1 & 0
\end{array}\right],
$$

and it can be exploited to define a reduced order observer (the subscript " $r$ " stands for "reduced"). Since the goal is the overshoot reduction, we use the technique in Proposition 3.2, with $\kappa_{M}=0.1$ and $L=$ $\left[\begin{array}{ll}0.0063 & 0.9015\end{array}\right]^{\top}$, to obtain (according to 4.7 and 4.8 )

$$
\begin{aligned}
K_{p} & =\left[\begin{array}{ll}
0.03680221 & 0.09245721
\end{array}\right], \\
\rho_{y} & =0.3435 \\
\bar{P}_{p} & =\left[\begin{array}{ll}
0.41279516 & 0.24891952 \\
0.24891952 & 0.89441164
\end{array}\right],
\end{aligned}
$$

where $\rho_{y}$ is the quantity to be minimized (according to the discussion after Proposition 3.2 . Then, by selecting $\tilde{\alpha}=10^{-12}, \rho=0.004$, $\epsilon=0.01, K_{y}=0.06, K_{x}=0, K_{c}=0, K_{\eta}=0.2$ and $N_{p}$ as in 4.13c, it is possible to define system (4.11) with the flow and jump sets in (4.13).

Figure 4.5 shows that the behavior of the hybrid closed-loop system does not change much compared to the full order case. The overshoot reduction is achieved and no faster rise time or other considerable effects coming from the observer can be observed, even for very large initial estimate errors.

\subsection{Fundamental properties of temporally regularized sys- tems}

When adding temporal regularization to a reset logic, the resulting dynamics generates solutions that behave more desirably in terms of avoiding Zeno behavior by way of enforcing a dwell-time condition between consecutive jumps (or resets). However, this positive feature goes with an undesirable possibility that solutions may flow even though the $x$ component is outside the set $\mathcal{F}$, because perhaps the timer $\tau$ is smaller than $\rho$. Due to this reason, it is harder to prove stability results for a temporally regularized system. In this section we establish a few fundamental conditions showing that, under certain reasonable assumptions, 

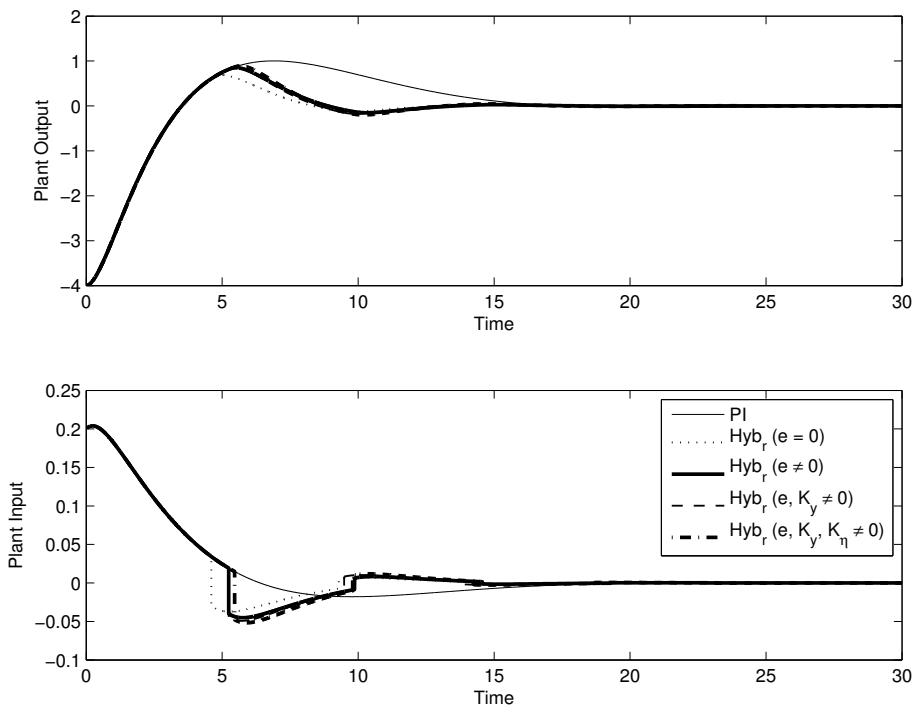

(a) Nominal case.
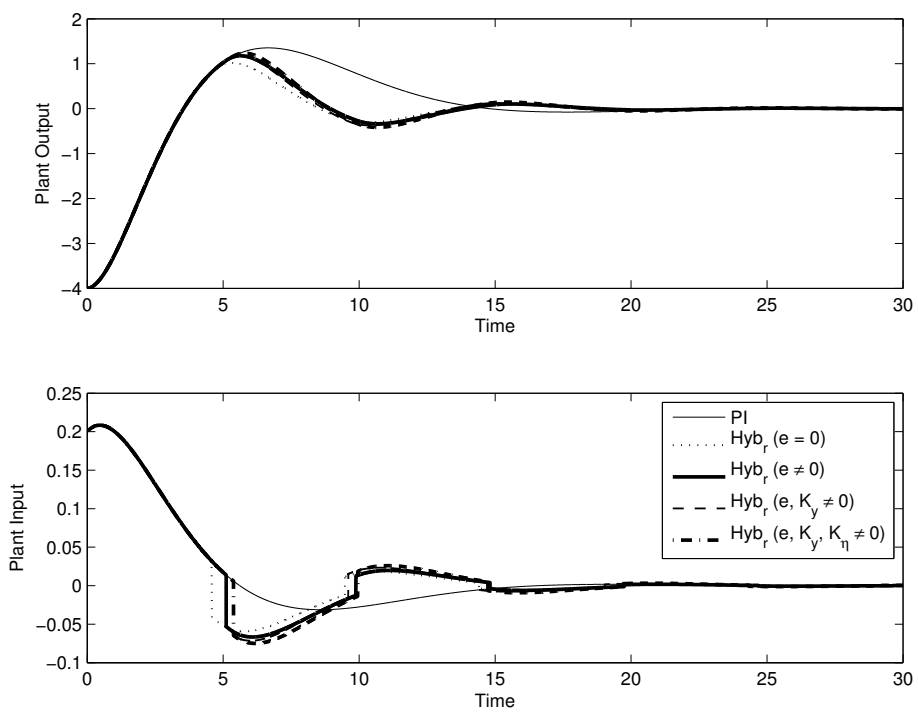

(b) Perturbed case.

Figure 4.5: Approximated reduced order observer case (Example of Section 4.3.2. 
one can reduce sufficiently the value of $\rho$, so that certain stability conditions holding before temporal regularization are preserved also after temporal regularization.

The results reported in this section appeared in Nešić et al. 2011 and represent the key tools for proving most of the results covered in Part III of this survey, and in particular those of Chapter 7 . They represent some useful alternatives to the proof techniques that appeared earlier in Nešić et al. [2008b], Zaccarian et al. [2005, 2007, 2011] to analyze reset systems with temporal regularization. These alternative techniques are instead surveyed in our Chapter 5. In particular, as compared to the results of Chapter 5, the theorems below exploit a strict Lyapunov decrease at jumps (which was not required in Nešić et al. [2008b] to compensate for a possible growth of $V$ outside the flow set $\mathcal{F}$. This was not possible in Nešić et al. 2008b because the Lyapunov functions were only required not to increase at jumps, thereby extra flow conditions had to be imposed on a slightly inflated version of the flow set. This new technique leads to a simpler proof than that of Nešić et al. 2008b. See Section 7.1 for a qualitative discussion of this fact.

\subsubsection{Lyapunov conditions for exponential and $\mathcal{L}_{2}$ stability}

Inspired by the nonlinear reset dynamics introduced in the previous chapter, let us consider the following peculiar structure for a nonlinear reset systems subject to an exogenous disturbance:

$$
\begin{aligned}
& \left\{\begin{array}{l}
\dot{\tau}=1-\mathrm{dz}\left(\frac{\tau}{\rho}\right), \quad x \in \mathcal{F} \text { or } \tau \in[0, \rho], \\
\dot{x}=f(x, d),
\end{array}\right. \\
& \left\{\begin{array}{l}
\tau^{+}=0, \\
x^{+}=g(x),
\end{array}\right.
\end{aligned}
$$

Then we formalize the requirement that there is a Lyapunov function for the $x$ dynamics of system (4.18), which decreases both during flows and along jumps.

Assumption 4.4. Given system (4.18), a suitable output $y$ satisfying $|y|^{2} \leq \lambda_{0}|x|^{2}$ and an integer $p \in[1,+\infty)$, the locally Lipschitz Lyapunov function $V: \mathbb{R}^{n} \rightarrow \mathbb{R}_{\geq 0}$ is such that there exist positive real 
numbers $\lambda_{i}, i=1, \ldots, 7$ and $\eta \in(0,1)$ such that for all $d$ :

$$
\begin{aligned}
\lambda_{1}|x|^{p} & \leq V(x) \leq \lambda_{2}|x|^{p} \\
\frac{\partial V(x)}{\partial x} f(x, d) & \leq \lambda_{3} V(x)+\lambda_{4}|x|^{p-1}|d|, \text { for a.a. } x \in \mathbb{R}^{n}, \\
\max _{v \in \partial V(x)} v^{\top} f(x, d) & \leq-\lambda_{5} V(x)-\lambda_{6}|y|^{p}+\lambda_{7}|d|^{p}, \quad \forall x \in \mathcal{F}, \\
V(g(x)) & \leq \eta V(x), \quad \forall x \in \mathcal{J} .
\end{aligned}
$$

where $\partial V(x)$ is the Clarke generalized gradient of $V(\cdot)$ at $x$ (see Clarke 1990 for its definition).

Remark 4.3. Note that global asymptotic stability of the origin of

$$
\left\{\begin{aligned}
\dot{x} & =f(x, d), & & x \in \mathcal{F}, \\
x^{+} & =g(x), & & x \in \mathcal{J},
\end{aligned}\right.
$$

(which has no temporal regularization) with $d=0$ is equivalent to 4.19c and 4.19d with $d=0$ and $V(\cdot)$ positive definite and radially unbounded. These conditions, a subset of the conditions in Assumption 4.4, are sufficient to establish semiglobal practical asymptotic stability for the system (4.20) augmented with temporal regularization, i.e., the system 4.18]. For details, see Goebel and Teel [2006] or Cai et al. 2008]. Here we also impose the stronger conditions (4.19a) and $4.19 \mathrm{~b}$ because we are interested in establishing global asymptotic stability results, in addition to properties of the system with disturbance inputs.

It should be noted that the way dynamics 4.18 is affected by the disturbance $d$ is quite peculiar, because its action only concerns the flow map and is completely absent in the jump map and in the jump/flow sets. This setting is motivated by the typical scenario in reset control where somehow the flowing nature of hybrid solutions is more relevant than the jumping one. In this setting, we measure the energy of the signal by only concentrating on its continuous-time nature. In particular, we use pseudo-norms of hybrid signals that only involve integrals and do not involve sums (for more general hybrid norms of signals, see Nešić et al. [2013]). These norms become even 
more relevant when considering temporally regularized systems whose complete solutions are always characterized by unbounded domains in the $t$ directions.

While we will define more carefully $t$ - $\mathcal{L}_{2}$ norms of hybrid signals later in Definition 5.1, let us provide here some relevant results that were reported in Nešić et al. 2011] and regarding some interesting connections between the Lyapunov conditions of Assumption 4.4 (which do not involve temporal regularization) and exponential and $t$ - $\mathcal{L}_{p}$ stability properties of the reset system with temporal regularization 4.18. When $p=2$, the definition of $t-\mathcal{L}_{p}$ stability is defined in Definition 5.1. For different values of $p \in[1, \infty]$, it is a straightforward generalization of that definition.

Theorem 4.5. Given an integer $p \in[1,+\infty)$, assume that there exists a function $V(\cdot)$ satisfying Assumption 4.4 . Then if $f(\cdot, \cdot)$ is continuous in its first argument, the reset system with temporal regularization 4.18 satisfies the following:

1. there exists $\bar{\rho}>0$ such that for any $\rho \in(0, \bar{\rho}]$ the origin of the $x$ dynamics with $d=0$ is exponentially stable;

2. the system is finite gain $t-\mathcal{L}_{p}$ stable from $d$ to $y$ and for any $\epsilon>0$, there exists $\bar{\rho}$ such that for all $\rho \leq \bar{\rho}$ the $t$ - $\mathcal{L}_{p}$ gain from $d$ to $y$ is upper bounded by $\left(\frac{\lambda_{7}}{\lambda_{6}}\right)^{1 / p}+\epsilon$.

Remark 4.4. The use of generalized gradients in Theorem 4.5 is motivated by the fact that the result is used in Theorems 7.177 .3 with locally Lipschitz Lyapunov functions. Note that it is not sufficient to impose the flow conditions 4.19c stated for the gradient of $V$ for almost all $x$. More specifically, for 4.19b it is sufficient to restrict the attention to almost everywhere because by continuity of $f$, for each disregarded point there is a full measure set of points where the condition holds. However, this reasoning does not hold for 4.19c where the condition is restricted to the set $\mathcal{F}$ for which no extra assumptions hold. In particular, one can construct defective cases with thin selections of $\mathcal{F}$, namely sets of measure zero, so that imposing a flow condition almost everywhere in $\mathcal{F}$ corresponds to not imposing it at all. 


\subsubsection{Stability of linear reset systems acting on cones}

An additional contribution from Nešić et al. 2011] addresses linear hybrid dynamics acting on jump and flow sets that are cones. Under this homogeneity property, based on Tuna and Teel [2006], parallel properties to those of standard linear systems can be established, establishing several equivalence between different notions of stability involving asymptotic stability, finite-gain $t-\mathcal{L}_{p}$ and $t-\mathcal{L}_{\infty}$ stability and also $t$-Input-to-State Stability (or $t$-ISS in short) defined as the existence of a class $\mathcal{K} \mathcal{L}$ function $\beta$ and a continuous nondecreasing nonlinear gain

$\gamma$ that is zero at zero, such that all solutions $x$ satisfy

$$
|x(t, j)| \leq \beta(|x(0,0)|, t)+\gamma\left(\|d\|_{\infty}\right), \forall(t, j) \in \operatorname{dom} x .
$$

More specifically, the following equivalences are proved:

- (local) asymptotic stability of the origin $\Leftrightarrow$ global exponential stability,

- global exponential stability $\Leftrightarrow$ finite gain $t-\mathcal{L}_{p}$ and finite gain $t-\mathcal{L}_{p}$ to $t$ - $\mathcal{L}_{\infty}$ stability from $d$ to $x$ with $p \in[1,+\infty)$

- global exponential stability $\Leftrightarrow$ finite gain exponential $t$-ISS from $d$ to $x$.

More specifically, we focus on the following class of temporally regularized linear reset systems:

$$
\begin{aligned}
& \begin{cases}\dot{\tau}=1-\mathrm{dz}\left(\frac{\tau}{\rho}\right), & x \in \mathcal{F} \text { or } \tau \in[0, \rho], \\
\dot{x}=A x+B d, & \end{cases} \\
& \left\{\begin{array}{l}
\tau^{+}=0, \\
x^{+}=G x,
\end{array}\right.
\end{aligned}
$$

where $x \in \mathbb{R}^{n}$ and $\tau \in \mathbb{R}$. We assume that 4.21 satisfies the following assumption.

Assumption 4.5. The sets $\mathcal{F}$ and $\mathcal{J}$ are closed nonempty subsets of $\mathbb{R}^{n}$. Moreover, they both are cones, namely, for each $\lambda>0$ and each $x \in \mathbb{R}^{n}, x \in \mathcal{F} \Rightarrow \lambda x \in \mathcal{F}$ and $x \in \mathcal{J} \Rightarrow \lambda x \in \mathcal{J}$. 
Let us conclude this chapter with the following robustness result for system 4.21) under Assumption 4.5.

Theorem 4.6. If Assumption 4.5 holds, then the following statements are equivalent:

1. the origin of the $x$ dynamics of 4.21 with $d=0$ is (locally) asymptotically stable;

2. the origin of the $x$ dynamics of 4.21 with $d=0$ is globally exponentially stable;

3. given $p \in[1,+\infty)$, system 4.21 is finite gain $t-\mathcal{L}_{p}$ stable and $t-\mathcal{L}_{p}$ to $t-\mathcal{L}_{\infty}$ stable from $d$ to $x$

4. system (4.21) is finite gain exponentially $t$-ISS from $d$ to $x$. 


\section{LMI-based stability and performance analysis}

\subsection{Introducing continuous-time $\mathcal{L}_{2}$ gains}

The material covered in the previous chapters often resulted in dynamics of reset closed loops that corresponded to linear flow and jump maps and flow/jump sets consisting in symmetric cones. Chapter 4 also discussed the importance of embedding temporal regularization in those dynamics. We focus in this chapter on a rather general class of temporally regularized systems that have been addressed, e.g., in Teel et al. 2013, Fichera et al. 2013, Nešić et al. 2008b, Zaccarian et al. 2011, Beker et al. 2001a, and that corresponds to

$$
\begin{aligned}
& \left\{\begin{array}{l}
\dot{x}=A x+B w \\
\dot{\tau}=1-\mathrm{dz}\left(\frac{\tau}{\rho}\right), \quad(x, \tau) \in \mathcal{C}
\end{array}\right. \\
& \left\{\begin{array}{l}
x^{+}=G x \\
\tau^{+}=0 \\
z=C_{z} x+D_{z w} w \\
y=C_{y} x+D_{y w} w
\end{array} \quad(x, \tau) \in \mathcal{D}\right.
\end{aligned}
$$

where $x \in \mathbb{R}^{n}$ is the ordinary state, $\tau \in \mathbb{R}$ is a dwell-time logic timer (with $\rho>0$ ), $w \in \mathbb{R}^{n_{w}}$ is an exogenous signal, $z \in \mathbb{R}^{n_{z}}$ is the performance output, $y \in \mathbb{R}^{n_{y}}$ is the measured output and $\mathcal{C}, \mathcal{D}$ are the flow 
and jump sets defined, respectively, as

$$
\begin{aligned}
\mathcal{C} & :=\{(x, \tau): x \in \mathcal{F} \text { or } \tau \in[0, \rho]\} \\
& =\{(x, \tau): x \in \mathcal{F}\} \cup\{(x, \tau): \tau \in[0, \rho]\}, \\
\mathcal{D} & :=\{(x, \tau): x \in \mathcal{J} \text { and } \tau \in[\rho, 2 \rho]\} \\
& =\{(x, \tau): x \in \mathcal{J}\} \cap\{(x, \tau): \tau \in[\rho, 2 \rho]\},
\end{aligned}
$$

with $\mathcal{F}$ and $\mathcal{J}$ symmetric cones defined by a matrix $M=M^{\top} \in \mathbb{R}^{n \times n}$ as

$$
\begin{aligned}
& \mathcal{F}:=\left\{x \in \mathbb{R}^{n}: x^{\top} M x \leq 0\right\}, \\
& \mathcal{J}:=\left\{x \in \mathbb{R}^{n}: x^{\top} M x \geq 0\right\} .
\end{aligned}
$$

Note that (5.1) and $\mathcal{C} \cup \mathcal{D}=\mathbb{R}^{n} \times[0,2 \rho]$ satisfy the Basic Assumptions of Goebel et al. 2009 and then it is easy to show that solutions exist for all initial conditions of $x \in \mathbb{R}^{n}$ and for all initial values in $[0,2 \rho]$ for the dwell time $\tau$. Since $\mathcal{C} \cup \mathcal{D}$ is forward invariant and no finite escape times are possible due to the linear flow map, then it follows that all maximal solutions are complete and we will refer in the following to asymptotic stability rather than pre-asymptotic stability (see Goebel et al. [2009], Goebel et al. 2012 for more details).

Similar to previous works, in this chapter we are concerned about the asymptotic behavior of $x$ and not of timer $\tau$. Therefore, we study stability properties of the compact

$$
\mathcal{A}=\{0\} \times[0,2 \rho] \subset \mathbb{R}^{n} \times[0,2 \rho] .
$$

Note that the set $[0,2 \rho]$ is forward invariant for $\tau$. Moreover, due to the dwell time, each maximal solution $\xi=(x, \tau)$ to (5.1)-(5.2) has a hybrid domain $E=\operatorname{dom}(\xi)$, which is unbounded in the ordinary time $t$ direction. More specifically, any two elements $(t, j),(s, k)$ of $E$ with $t>s$ satisfy the dwell-time condition (see Goebel et al. 2012, Cai et al. 2008 for details on dwell-time logic):

$$
\rho+t-s \geq \rho(j-k) .
$$

The hybrid system (5.1)-(5.2) is quite general and has been used in several works in the literature like Fichera et al. [2012b c, 2013, 
Zaccarian et al. 2005, 2011, Nešić et al. 2011, which justifies the interest of the results presented here.

The next remark states some important features of solutions to hybrid system (5.1)- 5.2 .

Remark 5.1. Consider any solution $\xi$ to system (5.1)-(5.2) and its jump times $t_{i}, i \in \operatorname{dom}(\xi) \subset \mathbb{N}$. Then:

i. $t_{i+1}-t_{i} \geq \rho$, for all $i \in \mathbb{N}, i \geq 1$. In particular, if $t_{i+1}-t_{i}>\rho$, $i \in \mathbb{N}, i \geq 1$, then $x(t, i) \in \mathcal{F}$ for all $t \in\left[t_{i}+\rho, t_{i+1}\right]$;

ii. in the first interval $\left[t_{0}, t_{1}\right]=\left[0, t_{1}\right]$, we have $t_{1}-t_{0} \geq \rho-\tau(0,0)$ and so it might happen that $t_{1}-t_{0}<\rho$ (note that this might also imply that $t_{1}=t_{0}=0$ if $\left.\tau(0,0) \geq \rho\right)$. Nevertheless, $x(t, 0) \in \mathcal{F}$ for all $t \in\left[\max \{0, \rho-\tau(0,0)\}, t_{1}\right]$;

iii. flow may occur in $\mathcal{J}$ due to the dwell-time logic;

iv. whenever $x \in \mathcal{F} \cap \mathcal{J}$ and $\tau \in[\rho, 2 \rho]$, the solution may either jump or flow.

Let us define now the $t$-decay rate property and the $t$ - $\mathcal{L}_{2}$ norm of a hybrid signal.

Definition 5.1. i. ( $t$-decay rate) Given a hybrid system, a compact set $\mathcal{A} \subset \mathbb{R}^{n}$ is uniformly globally exponentially stable with $t$-decay rate $\lambda>0$ if there exists a strictly positive real number $k$ such that each solution $x$ satisfies

$$
|x(t, j)|_{\mathcal{A}} \leq k \exp (-\lambda t)|x(0,0)|_{\mathcal{A}}, \quad \forall(t, j) \in \operatorname{dom}(x),
$$

where $\operatorname{dom}(x)$ denotes the hybrid time domain of the solution $x$.

ii. ( $t$ - $\mathcal{L}_{2}$ norm of a hybrid signal) For a hybrid signal $w$, with domain $\operatorname{dom}(w) \subset \mathbb{R}_{\geq 0} \times \mathbb{N}$, the $t-\mathcal{L}_{2}$ norm of $w$ is given by

$$
\|w\|_{2 t}=\left(\sum_{j \in \operatorname{dom}_{j}(w)} \int_{t_{j}}^{t_{j+1}}|w(t, j)|^{2} d t\right)^{\frac{1}{2}},
$$


where $\operatorname{dom}_{j}(w):=\{j \in \mathbb{N}:(t, j) \in \operatorname{dom}(w)$ for some $t \geq 0\}$ and with $t_{j+1}$ possibly being $\infty$ if $j \in \operatorname{dom}_{j}(w)$ and $(j+1) \notin \operatorname{dom}_{j}(w)$.

iii. $\left(w \in t-\mathcal{L}_{2}\right)$ For a hybrid signal $w$, with domain $\operatorname{dom}(w) \subset \mathbb{R}_{\geq 0} \times$ $\mathbb{N}_{\geq 0}$, we say $w \in t-\mathcal{L}_{2}$ whenever $\|w\|_{2 t}<\infty$. Moreover, for any pair $t_{1} \geq t_{2}$ such that $t_{1}, t_{2} \in \operatorname{dom}_{t}(w)$, we use $\left\|w\left[t_{1}, t_{2}\right]\right\|_{2}$ to denote the restriction of (5.6) to the corresponding subdomain.

Due to the dwell time in (5.1)- 5.2 (which guarantees condition (5.4), the $t$-decay rate property (5.5) implies uniform global exponential stability of the $x$ component of (5.1)-(5.2) in the hybrid sense (see Teel et al. [2013]). Furthermore, the dwell time is also a fundamental property that justifies the use of ordinary-time $\mathcal{L}_{2}$ norms defined in (5.6) (just as in Fichera et al. 2012c], Forni et al. [2011], Nešić et al. [2008b]). In particular, whenever the dwell-time condition (5.4) is satisfied, the definition in (5.6) essentially corresponds to the continuous-time $\mathcal{L}_{2}$ norm of the continuous-time signal $t \mapsto \xi_{t}(t)$ obtained by projecting on the ordinary time the hybrid $\operatorname{arc}(t, j) \mapsto \xi(t, j)$. Note that if the hybrid arc $\xi$ only flows, that is $\operatorname{dom}(\xi)=[0,+\infty) \times\{0\}$, then $(5.6)$ corresponds to the standard continuous-time $\mathcal{L}_{2}$ norm. Note also that $(5.6)$ is not a norm because, for example, a solution $\xi$ starting at a nonzero value at $(t, j)=(0,0)$ and jumping to zero at $(t, j+1)=(0,1)$ would satisfy $\|\xi\|_{2 t}=0$ (this is not the case for the hybrid norms introduced in Cai and Teel [2009], Nešić et al. [2013]). Nevertheless we call it norm throughout the paper due to the intuition that it generalizes the continuous-time norm.

A common performance index for dynamical systems consists in the worst case $t-\mathcal{L}_{2}$ norm amplification from an input $w$ and a performance output signal $z$ of interest. More precisely, we want to estimate the finite $t-\mathcal{L}_{2}$ gain of system (5.1)- 5.2 as defined next.

Definition 5.2. Consider the compact set $\mathcal{A}$ in (5.3). System (5.1)-(5.2) has finite $t-\mathcal{L}_{2}$ gain from $w$ to $z$ with gain (upper bounded by) $\gamma>0$, if any solution to (5.1)-(5.2) starting from $\mathcal{A}$ satisfies

$$
\|z\|_{2 t} \leq \gamma\|w\|_{2 t},
$$

for all $w \in t-\mathcal{L}_{2}$. 


\subsection{Lyapunov-based $t-\mathcal{L}_{2}$ stability conditions}

In this section, we provide sufficient conditions to establish $t$ - $\mathcal{L}_{2}$ gain performance bounds for system (5.1)-(5.2), relying on a Lyapunov function defined only in the $x$-state space direction. Our first theorem below, whose proof is reported in Fichera et al. 2015], corresponds to an enhancement of the Lyapunov conditions initially proposed in Nešić et al. [2008b]. In spite of their generality, the conditions given below may be too convoluted to digest, therefore we present next the simplified conditions that previously appeared in Nešić et al. [2008b that, despite some limitations as compared to those of Fichera et al. [2015], are general enough to allow deriving a number of useful Lyapunov constructions discussed at the end of this chapter. Suitable comparisons between the two versions of the stability theorem are reported next.

The intuitive goal of the results reported in the rest of this section is to establish if there exists a non-empty set of possible selections of the dwell-time parameter $\rho>0$ that guarantee global asymptotic stability of set $\mathcal{A}$ in $(5.3)$ for system $(5.1)-(5.2)$ with $w=0$ and we would like to build an estimate of the $t-\mathcal{L}_{2}$ gain from $w$ to $z$. The results are stated first considering a generic Lyapunov function and afterwards considering a quadratic Lyapunov function, which leads to a convenient convex linear matrix inequalities-based (LMI-based) formulation. The motivation to move in this direction is due to the example reported at the beginning of Section 5.4.3 discussing an exponentially stable reset system for which there does not exist a quadratic Lyapunov function.

Let us state the following statement which comprises a fairly general non-necessarily quadratic function $V$. The proof comprises several steps and is reported in Fichera 2013, Fichera et al. 2015.

Theorem 5.1. Consider system (5.1)-(5.2) and the following definitions

$$
\begin{aligned}
& \tilde{\mathcal{F}}=\left\{x \in \mathbb{R}^{n}: x^{\top} \tilde{M} x \leq 0\right\}, \\
& \tilde{\mathcal{F}}_{\epsilon}=\left\{x \in \mathbb{R}^{n}: x^{\top} \tilde{M} x-\epsilon x^{\top} x \leq 0\right\},
\end{aligned}
$$

with $\tilde{M}=\tilde{M}^{\top} \in \mathbb{R}^{n \times n}$ and $\epsilon>0$. Suppose there exist a continuously differentiable function $V: \mathbb{R}^{n} \rightarrow \mathbb{R}_{\geq 0}$ such that set $\tilde{\mathcal{F}}_{\epsilon}$ in $(5.9)$ satisfies 
$\mathcal{F} \subset \tilde{\mathcal{F}}_{\epsilon}$, positive real scalars $a_{1}, a_{2}, a_{3}, a_{4}, a_{5}, \bar{\gamma}$ and a nonnegative scalar $\underline{\rho}$ satisfying

$$
\begin{aligned}
& a_{1}|x|^{2} \leq V(x) \leq a_{2}|x|^{2}, \quad \forall x \in \mathbb{R}^{n}, \\
& \langle\nabla V(x), A x+B w\rangle+a_{3} V(x)+\frac{1}{\bar{\gamma}} z^{\top} z-\bar{\gamma} w^{\top} w<0, \\
& \forall x \in \tilde{\mathcal{F}}_{\epsilon} \backslash\{0\}, \forall w \in \mathbb{R}^{n_{w}}, \\
& V(G x) \leq \exp \left(a_{3} \underline{\rho}\right) V(x), \quad \forall x \in \mathcal{J}, \\
& G x \in \tilde{\mathcal{F}}, \quad \forall x \in \mathcal{J}, \\
& \langle\nabla V(x), A x+B w\rangle \leq a_{4} V(x)+a_{5}|x||w|, \\
& \forall x \in \mathbb{R}^{n}, \forall w \in \mathbb{R}^{n_{w}} .
\end{aligned}
$$

Then for any $\gamma$ satisfying

$$
\gamma \geq \bar{\gamma} \exp \left(\frac{a_{3} \underline{\rho}}{2}\right), \quad \gamma>\sqrt{2}\left|D_{z w}\right|
$$

there exists $\bar{\rho}>0$ such that for any $\rho \in(\underline{\rho}, \bar{\rho})$ :

1) when $w=0$, the set $\mathcal{A}$ in $(5.3)$ is globally asymptotically stable for the hybrid closed-loop system (5.1)- $(5.2)$;

2) when $w \neq 0$, with $w \in t$ - $\mathcal{L}_{2}$, the finite $t$ - $\mathcal{L}_{2}$ gain from $w$ to $z$ is less than or equal to $\gamma$, namely (5.7) holds for any solution to (5.1)-(5.2) from any initial condition $\xi(0,0)=(x(0,0), \tau(0,0)) \in \mathcal{A}$.

Theorem 5.1 establishes the existence of $\bar{\rho}$. A way to characterize an estimate of the value of $\bar{\rho}$ is based on suitable bounds reported next, that were first derived in [Nešić et al., 2008b. Theorem 1], and then refined in Fichera et al. 2015. Selecting $\rho_{1}^{\star}, \rho_{2}^{\star}$ and $\rho_{3}^{\star}$ as follows

$$
\begin{aligned}
\rho_{1}^{\star} & :=\varphi_{e}^{-1}\left(\frac{\epsilon}{|2(\tilde{M}-\epsilon I) A|}\right) \\
\rho_{2}^{\star} & :=\varphi_{1}^{-1}\left(\gamma^{2}-2\left|D_{z w}\right|^{2}\right), \quad \rho_{3}^{\star}:=\varphi_{2}^{-1}\left(\frac{\epsilon}{a_{2} \exp \left(a_{3} \underline{\rho}\right)}\right) \\
\varphi_{e}(s) & :=\frac{1}{2|A|}(\exp (2|A| s)-1) \\
\varphi_{1}(s) & :=\kappa_{1}(s)+\kappa_{2}(s)+\frac{2\left|C_{z}\right|^{2} s}{a_{1}}\left(1+\kappa_{1}(s)+\kappa_{2}(s)\right)
\end{aligned}
$$




$$
\begin{aligned}
& \varphi_{2}(s):=L_{1} \frac{s}{a_{1}}\left(1+\kappa_{1}(s)+\kappa_{2}(s)\right) \\
&+L_{2} \sqrt{\frac{s}{a_{1}}\left(1+\kappa_{1}(s)+\kappa_{2}(s)\right)} \\
& \kappa_{1}(s):=\exp \left(\frac{\bar{a}_{4}}{2} s\right) \kappa(s)+\frac{4 a_{1} \bar{a}_{4}}{\bar{a}_{5}^{2}} \kappa^{2}(s) \\
& \kappa_{2}(s):=\exp \left(\frac{\bar{a}_{4}}{2} s\right) \kappa(s)+\kappa^{2}(s) \\
& \kappa(s):=\frac{\bar{a}_{5}}{2} \sqrt{\frac{\exp \left(\bar{a}_{4} s\right)-1}{a_{1} \bar{a}_{4}}} \\
& L_{1}:=2|(\tilde{M}-\epsilon I) A|, \quad L_{2}:=2|(\tilde{M}-\epsilon I) B| \\
& \bar{a}_{4}:=a_{4}+a_{3}, \quad \bar{a}_{5}:=a_{5} \exp \left(a_{3} \underline{\rho}\right)
\end{aligned}
$$

where $\tilde{M}$ comes from $\tilde{\mathcal{F}}$ in $(5.8)$, then

- $\bar{\rho}=\rho_{1}^{\star}$ guarantees item 1 of Theorem 5.1

- $\bar{\rho}:=\min \left\{\rho_{2}^{\star}, \rho_{3}^{\star}\right\}$, satisfies item 2 of Theorem 5.1 .

Therefore the choice

$$
\bar{\rho}:=\min \left\{\rho_{1}^{\star}, \rho_{2}^{\star}, \rho_{3}^{\star}\right\}
$$

guarantees both items 1 and 2 of Theorem 5.1 .

Under certain restrictive conditions, we may present a simplified version of Theorem 5.1. which initially appeared in Nešić et al. 2008b and which can be seen then as a corollary of Theorem 5.1, as discussed next. We will make use of the corollary below for some of the statements reported in Section 5.4 .

Corollary 5.2. Consider the reset control system (5.1)-(5.2) and assume $D_{z w}=0$. Assume that there exist a locally Lipschitz function $V(x):=$ $x^{\top} P(x) x$, and strictly positive constants $a_{1}, a_{2}, \gamma, \varepsilon_{M}$ and $\varepsilon_{S}$, such that

1. $a_{1}|x|^{2} \leq V(x) \leq a_{2}|x|^{2}$ for all $x \in \mathbb{R}^{n}$,

2. $P(\lambda x)=P(x)=P^{\top}(x)>0$ for all $x \in \mathbb{R}^{n}$ and for all $\lambda \in \mathbb{R}$,

3. $\frac{\partial V(x)}{\partial x}(A x+B w)+\varepsilon_{S}|x|^{2}+\frac{1}{\gamma}|y|^{2}-\gamma|w|^{2}<0$, for almost all $x$ such that $x^{\top}\left(M-\varepsilon_{M} I\right) x \leq 0$, 


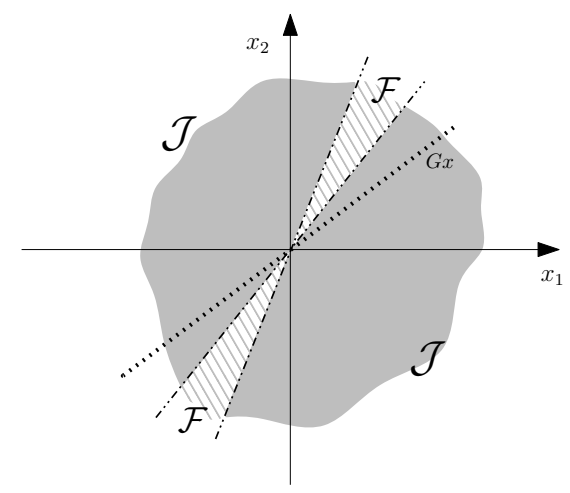

(a) sets $\mathcal{F}$ and $\mathcal{J}$ and image of jump map- (b) $G x \in \tilde{\mathcal{F}}$ and $\mathcal{F} \subset \tilde{\mathcal{F}}_{\epsilon}$ (notice also that ping $G x$.

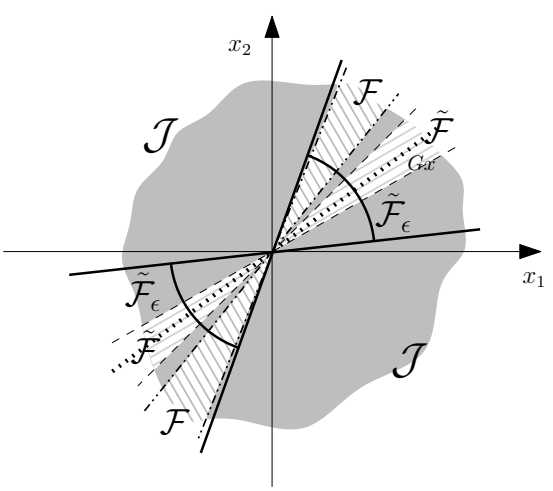

$\left.\tilde{\mathcal{F}} \subset \tilde{\mathcal{F}}_{\epsilon}\right)$.

Figure 5.1: Example where item 5 Corollary 5.2 is not satisfied (so that the corollary cannot be applied) and a possible selection of sets $\tilde{F}$ and $\tilde{\mathcal{F}}_{\epsilon}$ to apply Theorem $5.1\left(\tilde{\mathcal{F}}\right.$ is the conic region delimited by dashed lines and $\tilde{\mathcal{F}}_{\epsilon}$ is the conic region delimited by bold lines).

4. $V(G x)-V(x) \leq 0$ for all $x$ such that $x^{\top} M x \geq 0$.

5. $G x \in \mathcal{F}$ for all $x$ such that $x^{\top} M x \geq 0$.

Then there exists a small enough $\bar{\rho}>0$ such that for any fixed $\rho \in$ $(0, \bar{\rho})$, the reset control system (5.1)-(5.2) is exponentially stable and has a finite $t-\mathcal{L}_{2}$ gain from $w$ to $y$, which is smaller than $\gamma$.

Remark 5.2. The condition at item 2 corresponds to requiring that the Lyapunov function is homogeneous of degree two. The condition at item 3 corresponds to requiring that in a set that is slightly larger than the flow set the Lyapunov function is a disturbance attenuation Lyapunov function for the input $w$ and the output $y$. The condition at item 4 corresponds to requiring that the Lyapunov function does not increase along resets.

Theorem 5.1 is stronger than Corollary 5.2 in the following ways:

- The gain $D_{z w}$ is assumed to be zero in Corollary 5.2. Instead in Theorem 5.1 it should be noted that the second condition in 
(5.11) guarantees that the argument of $\varphi_{1}(\cdot)$ in (5.12a) is strictly positive and thus it guarantees also that $\bar{\rho}$ is strictly positive. Allowing $D_{z w} \neq 0$ is crucial in many relevant $\mathcal{H}_{\infty}$ type of performance goals arising in set-point regulation.

- Increase at jumps of the Lyapunov function $x \mapsto V(x)$ is allowed in Theorem 5.1, while it is not allowed in Corollary 5.2. By selecting a non-zero $\rho$ in (5.10c), growth at jumps is allowed and is balanced by a strengthened decrease condition during flow, imposed by the term $a_{3} P$ in $5.10 \mathrm{~b}$ (see Goebel et al., 2012, Proposition 3.29]). Corollary 5.2 requires non-increase at jumps thereby resulting often into numerical problems when specializing the stability conditions to numerical tests based on LMIs. Indeed, the non-increase requirement is often coupled with a partial reset action on the states, thereby resulting in thin feasibility sets that are prone to numerical issues.

- The requirement in item 5 of Corollary 5.2 is removed and replaced in Theorem 5.1 by the introduction of set $\tilde{\mathcal{F}}$ and its $\epsilon$ inflation, $\tilde{\mathcal{F}}_{\epsilon}$, in $(5.8)$ and $(5.9)$ respectively, which allow for more flexibility. Indeed the dwell-time perturbation upon the trajectories (see Remark 5.1) is addressed through these inflated sets. Moreover in Corollary 5.2, the jumps have to be mapped in the flow set, which is a requirement relaxed in Theorem 5.1. In particular, Figure 5.1(a) shows a case for which Corollary 5.2 cannot be applied. Figure 5.1(b) instead, shows a possible selection of sets $\tilde{\mathcal{F}}$ and $\tilde{\mathcal{F}}_{\epsilon}$ so that Theorem 5.1 can still be applied. Note that the situation of Figure 5.1(a) is quite common in certain reset control systems where $G$ maps to the boundary of $\mathcal{F}$. Clearly, item 5 of Corollary 5.2 is a particular case of Theorem 5.1 and it can be retrieved by selecting $\tilde{\mathcal{F}}=\mathcal{F}$ (namely, $\tilde{M}=M$ ). Finally, we notice that $\tilde{\mathcal{F}} \subset \tilde{\mathcal{F}}_{\epsilon}$ always holds.

Remark 5.3. A generic choice of the parameters $\epsilon$ and $\gamma$ in Theorem 5.1 does not always guarantee that the set of suitable $\rho$ (namely, $(\underline{\rho}, \bar{\rho})$ ) is non empty. In particular, whenever $\underline{\rho}$ is non-zero (namely, a growth at jumps is admitted) there is no guarantee a priori that $\underline{\rho}<\bar{\rho}$ (for 
example, when using the construction for $\bar{\rho}$ in $(5.12)$ ). Therefore, whenever $\underline{\rho}>\bar{\rho}$, the set $(\underline{\rho}, \bar{\rho})$ is empty. However, whenever $\underline{\rho}=0$ (namely no increase at jumps is allowed), since $\rho_{1}^{\star}, \rho_{2}^{\star}$ and $\rho_{3}^{\star}$ are strictly positive (see the definitions in (5.12), then $\bar{\rho}>0$ and the set of suitable $\rho$ is $(\underline{\rho}, \bar{\rho})=(0, \bar{\rho})$ and is non empty. Moreover, we emphasize that $\varphi_{e}(\cdot), \varphi_{1}(\cdot)$ and $\varphi_{2}(\cdot)$ defined in 5.12 are class $\mathcal{K}_{\infty}$ functions and so also their inverses, which in particular, depend either on $\gamma$ or on $\epsilon$. Therefore, since $\bar{\rho}$ is the minimum of these last class $\mathcal{K}_{\infty}$ functions, by enlarging $\epsilon$ and/or $\gamma$, we may always obtain $\underline{\rho}<\bar{\rho}$.

Remark 5.4. There are some special cases for which Theorem 5.1 can be strengthened:

1. Global flow condition: suppose that (5.10b holds globally for all $x \in \mathbb{R}^{n} \backslash\{0\}$ (consider, for instance, the case where $\epsilon \geq \lambda_{\max }(\tilde{M})$, namely, $\tilde{\mathcal{F}}_{\epsilon}=\mathbb{R}^{n}$ ). Therefore even when $D_{z w} \neq 0$, the second condition of (5.11) is not needed and $\gamma=\bar{\gamma} \exp \left(\frac{a_{3} \underline{\rho}}{2}\right)$. In this case, the set $(\underline{\rho}, \bar{\rho})$ can always be selected as non empty, by arbitrarily enlarging $\bar{\rho}$.

2. Exponential stability: item 1 of Theorem 5.1 establishes global asymptotic stability of set $\{0\} \times[0,2 \rho]$. To prove global exponential stability, we should require a further decrease term in 5.10b). In particular, the term $a_{3} V(x)$ in $5.10 \mathrm{~b}$ is needed to compensate for the eventual growth at jumps due to $\rho$. Nevertheless, replacing $a_{3} V(x)$ in $\left.5.10 \mathrm{~b}\right)$ by $\left(a_{3}+\eta\right) V(x)$ with $\eta>0$, then global exponential stability of the attractor $\mathcal{A}$ can be ensured even when $\underline{\rho} \neq 0$. On the other hand, whenever $\underline{\rho}=0$, item 1 of Theorem 5.1 provides global exponential stability of set $\{0\} \times[0,2 \rho]$, because $a_{3} V(x)$ does not have to compensate for any growth at jumps.

Notice that whenever $D_{z w}=0$ and $\underline{\rho}=0$, then from (5.11) the result in Corollary 5.2 with $\gamma=\bar{\gamma}$ is recovered.

\subsection{LMI-based $\mathcal{L}_{2}$ stability conditions}

A relevant consequence of Theorem 5.1 is the following proposition that requires the existence of a single quadratic Lyapunov function $V(x)=$ 
$x^{\top} P x, P=P^{\top}>0$ satisfying the conditions of Theorem 5.1, namely the same quadratic function satisfies the flow condition in the flow set and the jump condition in the jump set. While being conservative, this special case is of interest because it leads to convenient convex formulations, especially relevant when performing reset control designs, such as those reported in the next chapter.

Proposition 5.1. Suppose there exist matrices $P=P^{\top}>0, \tilde{M}=\tilde{M}^{\top}$, non-negative scalars $\underline{\rho}, \tau_{S}, \tau_{F}, \tau_{C}, \tau_{R}$ and positive scalars $\epsilon, \bar{\gamma}, a_{3}$ such that

$$
\begin{array}{lcc}
\left(\begin{array}{ccc}
A^{\top} P+P A+a_{3} P-\tau_{S}(\tilde{M}-\epsilon I) & P B & C_{z}^{\top} \\
B^{\top} P & -\bar{\gamma} I & D_{z w}^{\top} \\
C_{z} & D_{z w} & -\bar{\gamma} I
\end{array}\right) & <0, \\
G^{\top} P G-\exp \left(a_{3} \underline{\rho}\right) P+\tau_{R} M & & \\
\tilde{M}-\tau_{F} M & & \leq 0, \\
G^{\top} \tilde{M} G+\tau_{C} M & & \leq \epsilon I, \\
& & \leq 0 .
\end{array}
$$

Then for any $\gamma$ satisfying (5.11), there exists $\bar{\rho}>0$ such that for any $\rho \in(\underline{\rho}, \bar{\rho})$ :

1 ) the set $\mathcal{A}$ in $(5.3)$ is globally exponentially stable for the hybrid closed-loop system (5.1)-(5.2), with $w=0$;

2) the $t-\mathcal{L}_{2}$ gain from $w$ to $z$ is less than or equal to $\gamma$, for all $w \in t-\mathcal{L}_{2}$.

For this quadratic case, the generalization in Proposition 5.1 (which comes from Fichera et al. 2015) appears relevant, as compared to the conditions previously published in Nešić et al. 2008b, derived from the above-mentioned Corollary 5.2 , which are reported below and can be well seen as a corollary of the above statement. A slight novelty as compared to Nešić et al. 2008b is the third condition, ensuring item 5 of Corollary 5.2 .

Corollary 5.3. Nešić et al. 2008b Consider the reset control system (5.1)- 5.2 with $D_{z w}=0$. Assume the following linear matrix inequali- 
ties in the variables $P=P^{\top}>0, \tau_{S}, \tau_{R}, \tau_{C} \geq 0, \gamma>0$ are feasible:

$$
\begin{aligned}
& {\left[\begin{array}{ccc}
A^{\top} P+P A-\tau_{S} M & P B & C_{z}^{\top} \\
\star & -\gamma I & 0 \\
\star & \star & -\gamma I
\end{array}\right]<0,} \\
& G^{\top} P G-P+\tau_{R} M \leq 0, \\
& G^{\top} M G+\tau_{C} M \leq 0 .
\end{aligned}
$$

Then there exists a small enough $\bar{\rho}>0$ such that for any fixed $\rho \in$ $(0, \bar{\rho})$, the reset control system (5.1)-(5.2) is exponentially stable and has a finite $t-\mathcal{L}_{2}$ gain from $w$ to $y$ which is smaller than $\gamma$.

Proposition 5.1 provides a simple tool to solve conditions 5.10 thanks to the use of the LMI-based framework (Boyd et al. 1994]). Indeed conditions (5.13) are linear except for $a_{3}, \tau_{S}$ in (5.13a) and the exponential term in $(5.13 \mathrm{~b})$. Therefore to perform the $t-\mathcal{L}_{2}$ analysis, $a_{3}$ and $\rho$ have to be imposed a priori and a line search upon $\tau_{S} \geq 0$ may need to be done. Although this may seem restrictive as compared to the convex formulation in Corollary 5.3, the reason why we insist on the more general formulation of Proposition 5.1 is to gain important degrees of freedom in the LMI optimization. Indeed practical experience reveals that the conditions in Corollary 5.3 are prone to numerical problems when looking at situations (such as those of Chapter 6) where some part of the state remains unchanged across jumps. In those cases, allowing for a slight increase of $V$ across jumps results in numerically more tractable conditions. This can be accomplished by fixing small values of $\underline{\rho}$ and $a_{3}$ and then solving the arising LMI conditions with a line search on $\tau_{S}$.

Remark 5.5. Let us point out the two cases below. Conditions 5.13 can be simplified by considering $a_{3}=0$ and/or $\epsilon=0$.

i. Case with $a_{3}=0$ : if $\underline{\rho}=0$, then $a_{3}$ does not need to compensate for any growth at jumps and therefore it can be selected arbitrarily small because of the strict inequality in (5.13a).

ii. Case with $\epsilon=0$ : if $\tau_{S}=1$ and $\tilde{M} \leq \tau_{F} M$ then (5.13c) holds for any $\epsilon>0$ and $\epsilon$ can be selected arbitrarily small because of the strict inequality in 5.13a. 
Moreover, whenever both cases apply, then Proposition 5.1 recovers the LMI conditions of Corollary 5.3 and one gets also that set $(\underline{\rho}, \bar{\rho})=(0, \bar{\rho})$ is always non-empty (see also a similar comment in Remark 5.3).

\subsection{Application to SISO control loops with FORE}

\subsubsection{Expression of the closed-loop matrices}

Consider a strictly proper SISO linear plant whose dynamics is described by

$$
\mathcal{P}\left\{\begin{aligned}
\dot{x}_{p} & =A_{p} x_{p}+B_{p u} u+B_{p d} d, \\
y & =C_{p} x_{p},
\end{aligned}\right.
$$

where $u$ is the control input, $d$ is a disturbance input, $y$ is the measured plant output and $A_{p}, B_{p u}, B_{p d}$ and $C_{p}$ are matrices of appropriate dimensions.

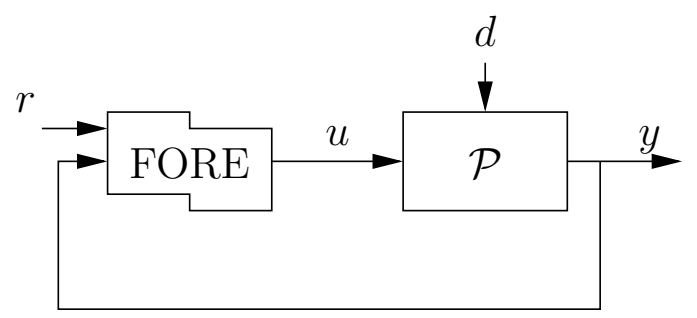

Figure 5.2: A linear plant controlled by a FORE.

For the plant (5.15), assume that a FORE control system is designed, according to Figure 5.2, where the FORE element is described by the following dynamics:

$$
\begin{aligned}
\text { FORE } \begin{cases}\dot{x}_{c}=a_{c} x_{c}+e, & e x_{c} \geq 0 \\
x_{c}^{+}=0, & e x_{c} \leq 0,\end{cases} \\
\text { Interconnection }\left\{\begin{array}{l}
u=k x_{c}, \\
e=r-y,
\end{array}\right.
\end{aligned}
$$

where $r \in \mathbb{R}$ is a reference signal. Moreover, $k$ denotes the loop gain and $a_{c} \in \mathbb{R}$ denotes the pole of the FORE. Note that $a_{c}$ can be any 
number (including positive ones) while $k$ should be positive. For example, choosing $k=1$ and $a_{c}=0$ corresponds to implementing in the FORE the well known Clegg integrator introduced in Clegg [1958] and discussed in Chapters 1 and 2 .

The overall closed-loop system augmented with temporal regularization can be written in the form (5.1), where $x=\left[x_{p}^{\top} x_{c}\right]^{\top}$ and $z=y$, while the matrices in (5.1) correspond to

$$
\begin{aligned}
& {\left[\begin{array}{c|c|c}
A & B_{d} & B_{r} \\
\hline C_{z} & D_{z d} & D_{z r}
\end{array}\right]=\left[\begin{array}{cc|c|c}
A_{p} & B_{p u} k & B_{p d} & 0 \\
-C_{p} & a_{c} & 0 & 1 \\
\hline C_{p} & 0 & 0 & 0
\end{array}\right]} \\
& {[G \mid M]=\left[\begin{array}{cc|cc}
I & 0 & 0 & C_{p}^{\top} \\
0 & 0 & C_{p} & 0
\end{array}\right]}
\end{aligned}
$$

where we may then either select $w=d$ or $w=r$ (and $B=B_{d}$ or $B=$ $B_{r}$, respectively) to alternatively study the effect of the disturbance or the effect of the reference input on the performance output.

\subsubsection{Explicit Lyapunov functions for FORE controlling an integra- tor}

When focusing on the specific interconnection of Figure 5.2, the Lyapunov-based results of Theorem 5.1 can be exploited to analytically construct a Lyapunov function by first focusing on the flow set and designing a suitable shape to guarantee decrease along flows, and then patching the Lyapunov level sets with an extra piece which satisfies the jump condition in the rest of the state-space. We address here the simple, yet very relevant case of a FORE connected to an integrator, which has been thoroughly discussed in Chapter 2 for the special case of $a_{c}=0$. This planar system has been widely studied in the literature and its improved $\mathcal{L}_{2}$ performance properties are here characterized by way of a pair of analytic Lyapunov functions. The bounds corresponding to equation $(5.20)$ are graphically represented in Figure 5.8 in Section 5.5, where they are compared to the bounds obtained by using the numerical optimization tools of Section 5.4.3.

Theorem 5.4. Given any $a_{c} \in \mathbb{R}$, c onsider the closed-loop between

$$
\dot{y}=u+d,
$$


and the FORE (5.16), 5.17) with $k=1$ and with temporal regularization. Then there exists a Lyapunov function $V(\cdot)$ which satisfies the conditions in Corollary 5.2 and, as $\rho \rightarrow 0$, gives a bound (depending on the FORE's pole $a_{c}$ ) for the $t-\mathcal{L}_{2}$ gain estimate from $d$ to $y$ arbitrarily close to the following value:

$$
\gamma\left(a_{c}\right) \leq \begin{cases}\frac{2}{\left|a_{c}\right|}+\left|a_{c}\right|, & \text { if } a_{c}<0, \\ \max \left\{\frac{\pi}{2}, \frac{2 \pi}{4+\pi a_{c}}\right\}, & \text { if } a_{c}>-\frac{4}{\pi}\end{cases}
$$
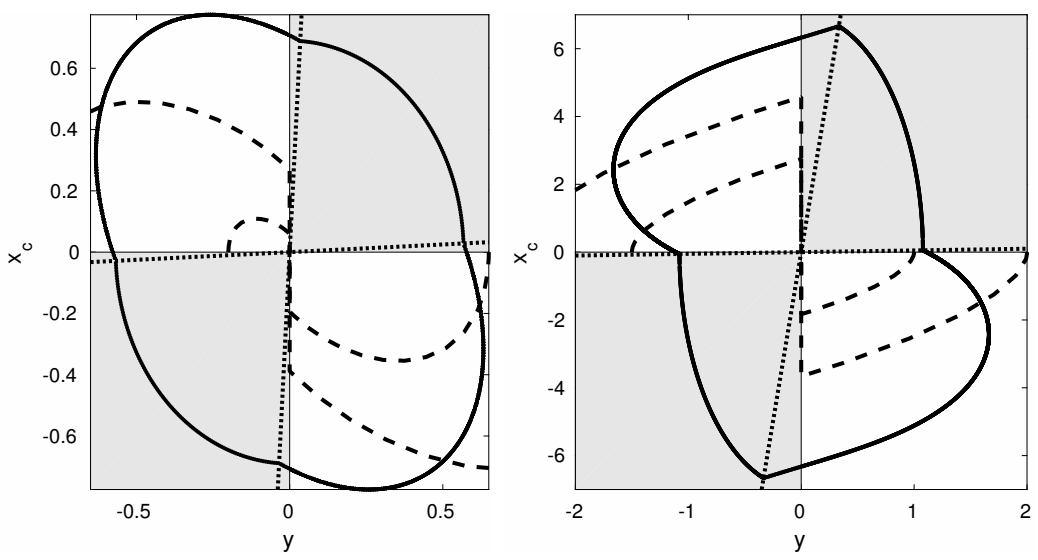

Figure 5.3: Level sets of the two Lyapunov functions proposed in Theorem 5.4 (solid) and trajectories of the closed-loop selecting $\theta_{\epsilon}=0.05$ (dashed). Left: $a_{c}=-1$. Right: $a_{c}=1$.

The proof relies on Corollary 5.2 and is only sketched here (see $\mathrm{Za}$ ccarian et al. 2006 for details). It consists in proposing two candidate Lyapunov functions (one for each one of the two bounds in (5.20) ). In particular, as illustrated in the level sets reported in Figure 5.3 , both functions are defined as follows:

$$
V(x):= \begin{cases}V_{f}(x), & \text { if } x^{\top} M_{\theta_{\varepsilon}} x \geq 0, \\ x^{\top} \hat{P} x, & \text { if } x^{\top} M_{\theta_{\varepsilon}} x \leq 0,\end{cases}
$$

where $x:=\left(y, x_{c}\right), \theta_{\epsilon}$ is a small enough angle and $M_{\theta_{\epsilon}}:=$ $\left[\begin{array}{cc}\sin \left(2 \theta_{\epsilon}\right) & -1 \\ -1 & \sin \left(2 \theta_{\epsilon}\right)\end{array}\right]$ is associated with the white regions in Figure 5.3 , corresponding to the second and fourth quadrant inflated by an angle $\theta_{\epsilon}$. 
Given the selection (5.21), each bound in (5.20) arises from selecting a smooth $V_{f}(\cdot)$ which is positive definite in $\left\{x: x^{\top} M_{\theta_{\varepsilon}} x \geq 0\right\}$. Moreover, the matrix $\hat{P}$ is selected so that continuity of $V(\cdot)$ is ensured and the jump condition at item 4 of Corollary 5.2 is satisfied (this is always possible for any smooth $V_{f}(\cdot)$ which is positive definite in $\left\{x: x^{\top} M_{\theta_{\varepsilon}} x \geq 0\right\}$ and for a small enough $\theta_{\varepsilon}$ ). In particular, we pick $\hat{P}$ diagonal with the following diagonal entries:

$$
\left[\begin{array}{l}
\hat{p}_{1} \\
\hat{p}_{2}
\end{array}\right]=\left[\begin{array}{cc}
\cos ^{2} \theta_{\varepsilon} & \sin ^{2} \theta_{\varepsilon} \\
\sin ^{2} \theta_{\varepsilon} & \cos ^{2} \theta_{\varepsilon}
\end{array}\right]^{-1}\left[\begin{array}{l}
v_{1} \\
v_{2}
\end{array}\right]
$$

where $v_{1}:=V_{f}^{2}\left(\cos \theta_{\varepsilon}, \sin \theta_{\varepsilon}\right)$ and $v_{2}:=V_{f}^{2}\left(\sin \theta_{\varepsilon}, \cos \theta_{\varepsilon}\right)$ are the values of $V_{f}(\cdot)$ on the patching hyperplanes.

Based on (5.21), to prove the first bound in (5.20), the following selection is made:

$$
V_{f}(x):=x^{\top} P x:=x^{\top}\left[\begin{array}{cc}
-\frac{2+a_{c}^{2}}{a_{c}} & 1 \\
1 & -\frac{2}{a_{c}}
\end{array}\right] x,
$$

while for the second bound in $(5.20)$, the function $V_{f}$ is selected in terms of a polar coordinate system $(r, \theta)$ satisfying $\left(y, x_{c}\right)=(r \cos \theta, r \sin \theta)$ :

$$
V_{f}:= \begin{cases}\frac{1}{2} r^{2}\left(\theta-\frac{\pi}{2}+\frac{1}{2} \sin 2 \theta+\varphi_{\epsilon}(\theta)\right), & \text { if } \theta \in\left[\frac{\pi}{2}-\theta_{\varepsilon}, \pi+\theta_{\varepsilon}\right], \\ \frac{1}{2} r^{2}\left(\theta-\frac{3 \pi}{2}-\frac{1}{2} \sin 2 \theta+\varphi_{\epsilon}\left(\theta-\frac{\pi}{2}\right)\right), \text { if } \theta \in\left[\frac{3 \pi}{2}-\theta_{\varepsilon}, 2 \pi+\theta_{\varepsilon}\right],\end{cases}
$$

where $\varphi_{\epsilon}(\theta)=\epsilon\left(\frac{1}{2 \max \left\{\left|a_{c}\right|, 1\right\}}-\sin \theta \cos \theta\right)$ and $\epsilon$ is a small enough positive constant.

Remark 5.6. (Comparing analytic and numerical bounds) In Figure 5.8 reported later in Section 5.5, the $t-\mathcal{L}_{2}$ bounds obtained in Theorem 5.4 for different values of $a_{c}$ are compared to the numerical bounds obtained by applying the numerical results proposed in Section 5.4.3. Note that the numerical bounds are always tighter than the analytic ones, however, the relevance of the analytic results stands in the fact that exponential stability and a bound on the $t-\mathcal{L}_{2}$ gain is proved for all values of $a_{c}$, whereas numerical tools inevitably lead to infeasibility, due to numerical problems, for positive values of $a_{c}$ that become too large. 
Remark 5.7. (A lower bound on the $t-\mathcal{L}_{2}$ gain for $a_{c}=0$ ) An interesting question corresponds to asking how tight the analytic and numerical bounds in Figure 5.8 are. A partial answer to this question is given by the following result which establishes that for the case $a_{c}=0$ (namely, the Clegg integrator discussed in Chapter 1), the gain is not smaller than $\sqrt{\pi / 8} \approx 0.626$, which coincides with the star reported in Figure 5.8

To show this property for the gain, consider the closed loop without temporal regularization (the extension is trivial) between the Clegg integrator introduced in (1.1) and a purely integrating plant (see Chapter 2 for ample discussions about this closed loop). Its equations can be written as

$$
\left\{\begin{aligned}
\dot{y} & =x_{c}+d \\
\dot{x}_{c} & =-y
\end{aligned}\right.
$$

and select the following initial conditions $y(0) \in\left(\frac{\sqrt{2}}{2}, 0\right), x_{c}(0)=$ $-y(0)$. Then select the following disturbance:

$$
d(t)=\left\{\begin{array}{cc}
2 \exp (t) y(0) & t \in\left[0, t^{*}\right], \\
0 & t>t^{*},
\end{array}\right.
$$

where $t^{*}:=\ln \left(\frac{\sqrt{2}}{-2 y(0)}\right)$. Then it is immediate to check that

$$
\begin{aligned}
\|d\|_{2} & =\sqrt{4 \cdot \frac{1}{2}\left[\exp \left(2 t^{*}\right)-1\right] y^{2}(0)} \\
& \leq \sqrt{2}\left|\exp \left(t^{*}\right) y(0)\right|=1 .
\end{aligned}
$$

and that in the limit as $y(0) \rightarrow 0,\|d\|_{2} \rightarrow 1$. Then, by substituting in (5.24) and considering that $x_{c}(t)=-\exp (t) y(0)$, the following holds:

$$
y(t)=\left\{\begin{array}{cc}
\exp (t) y(0) & t \in\left[0, t^{*}\right], \\
-\cos \left(t-t^{*}+\pi / 4\right) & t \in\left[t^{*}, t^{*}+\pi / 4\right] .
\end{array}\right.
$$

It follows that in the first time interval $\left[0, t^{*}\right]$, since $y(t)=0.5 d(t)$, we have

$$
\left\|y_{\left[0, t^{*}\right]}\right\|_{2}^{2}=\frac{1}{2}\left[\exp \left(2 t^{*}\right)-1\right] y^{2}(0)
$$


and that in the limit as $y(0) \rightarrow 0,\left\|y_{\left[0, t^{*}\right]}\right\|_{2}^{2} \rightarrow 1 / 4$. For the remaining time interval, we have

$$
\begin{aligned}
\left\|y_{\left[t^{*}, t^{*}+\pi / 4\right]}\right\|_{2}^{2} & =\int_{\pi / 4}^{\pi / 2} \cos ^{2}(\tau) d \tau \\
& =\left|\frac{1}{2} t+\frac{1}{4} \sin (2 t)\right| \\
& =\frac{\pi}{4}-\frac{\pi}{8}-\frac{1}{4} .
\end{aligned}
$$

Since the state is reset to zero and remains there after $t^{*}+\pi / 4$, then, in the limit as $y(0) \rightarrow 0$, we have

$$
\|y\|_{2} \rightarrow \sqrt{\frac{\pi}{8}}=\sqrt{\frac{\pi}{8}}\|d\|_{2}
$$

which proves the claim.
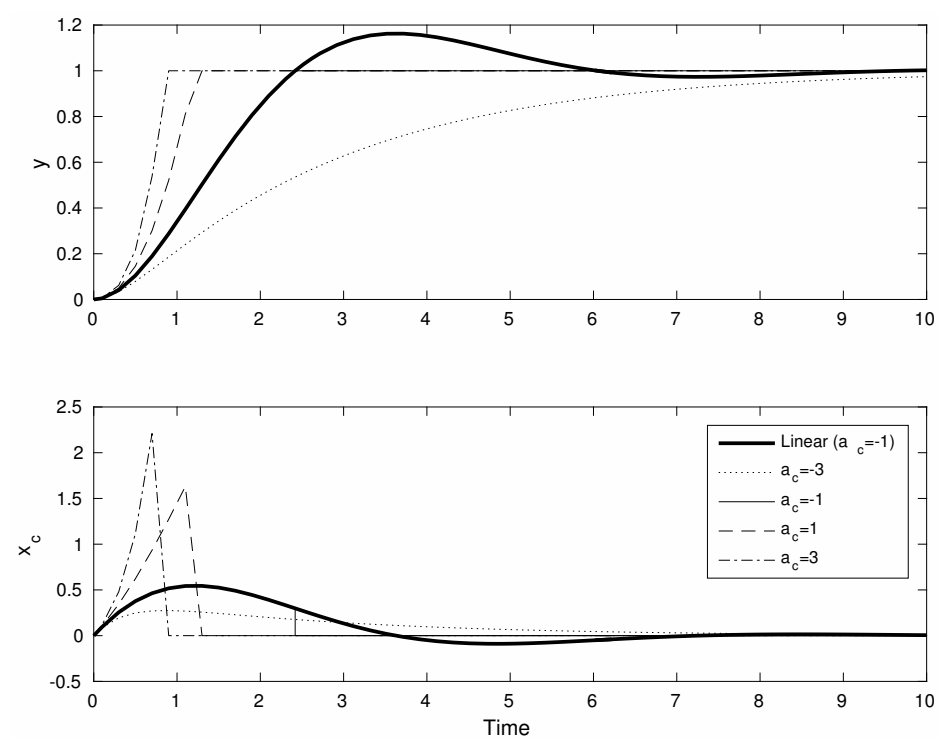

Figure 5.4: Simulations of step responses of closed loops between FOREs and an integrator for different values of $a_{c}$.

Remark 5.8. (From intuition to formalization) The results of Theorem 5.4 correspond to the mathematical formalization of the following 
intuitive reasoning about the closed-loop behavior of the control system of Figure 5.2. Since the plant is an integrator, then the linear part of the control system will always correspond to trajectories that spiral around the origin of the phase plane. Having $a_{c}=0$ will correspond to closed trajectories (circles in the phase plane), $a_{c}<0$ will lead to exponentially stable trajectories spiraling inward toward the origin and $a_{c}>0$ will lead to exponentially unstable trajectories spiraling outward toward infinity. When resets come to place, any of these stable and unstable trajectories will be blocked when they approach the second and fourth quadrant, and will be reset to zero, no matter what the value of $a_{c}$ is. Therefore, the conclusion about exponential stability established in Theorem 5.4 holds. Let us consider now the bounds on the $t$ - $\mathcal{L}_{2}$ gain from $d$ to $y$. Large negative values of $a_{c}$ will correspond to exponentially stable branches of trajectories that move very slowly toward the resetting quadrants, therefore the $t-\mathcal{L}_{2}$ gain of the corresponding closed loops will be larger and larger as $a_{c}$ becomes more and more negative (see also the very left of Figure 5.8). The decreasing trend of the gain as $a_{c}$ approaches zero only occurs up to a certain point in the linear case because the linear trajectories approach the unstable cases (occurring with $a_{c}>0$ ). Conversely, in the reset case, the branches approaching the reset quadrants become increasingly fast and steep, even for positive values of $a_{c}$. The corresponding gain becomes then smaller and smaller. This trend is easily understood by inspecting the simulations of Figure 5.4, where several step responses (corresponding to increasing values of $a_{c}$ ) are reported. From these simulations it becomes evident that as $a_{c}$ approaches $+\infty$, the step responses approach a step output (so that the gain is expected to approach zero) because they correspond to an increasingly fast exponentially unstable branch up to the desired set-point, followed by a constant branch. The decreasing trend of the gain as $a_{c}$ approaches $+\infty$ is confirmed by the numerical results reported in Figure 5.8 of Section 5.5 with reference to Example 5.2, whereas the bound provided by our Lyapunov approach is non decreasing. An analytical proof of the fact that the gain approaches zero as $a_{c}$ approaches $+\infty$ has been given in Nešić et al. 2008a, 2011] when using a slightly modified closed loop which ensures strict decrease 
along jumps thereby relaxing the requirement to inflate the set where the flow condition holds.

\subsubsection{Piecewise quadratic construction for SISO control loops with FORE}

Although the LMI results in Proposition 5.1 can be a useful tool for establishing the stability and performance of a FORE control system, there are severe limitations to what can be shown using that convex relaxation of the condition in Theorem 5.1. mainly arising from the conservativeness associated with the use of quadratic Lyapunov functions 1 . Indeed, it is possible to show that those quadratic Lyapunov conditions are never feasible if the FORE element is not exponentially stable. As an example of this, let us focus on the simpler conditions of Corollary 5.3 and corresponding to (5.14) and consider the case of a FORE controlling an integrator, which has been subject of great attention over the years (see the discussion in Chapter 2). For the sake of generality let us consider any positive loop gain $k>0$. With all the external signals at zero, we have during flow:

$$
\begin{aligned}
\dot{y} & =k x_{c}, \\
\dot{x}_{c} & =a_{c} x_{c}-y .
\end{aligned}
$$

Consider a quadratic Lyapunov function $V(x)=\left[\begin{array}{c}y \\ x_{c}\end{array}\right]^{\top}\left[\begin{array}{cc}p_{11} & \frac{p_{12}}{2} \\ \frac{p_{12}}{2} & p_{22}\end{array}\right]\left[\begin{array}{c}y \\ x_{c}\end{array}\right]$ and its derivative along the system flow:

$$
\dot{V}(x)=-p_{12} y^{2}+\left(p_{12} k+2 p_{22} a_{c}\right) x_{c}^{2}+\left(2 p_{11} k+p_{12} a_{c}-2 p_{22}\right) x_{c} y,
$$

Due to the shape of the flow set, we need $\dot{V}(x)<0$ both when $x_{c}=0$, which requires $p_{12}>0$ and when $y=0$, which requires $p_{12}<0$ because $k, p_{22}>0$ and $a_{c} \geq 0$ by assumption. The result follows from the contradiction that $p_{12}$ needs to be both positive and negative.

Based on the above reasoning, the convex LMI-based constructions in Proposition 5.1 and Corollary 5.3 do not appear to be good to prove useful stability and performance properties induced by resets on an otherwise unstable linear closed-loop. Indeed, with an integrating plant the

\footnotetext{
${ }^{1}$ The conservativeness of quadratic Lyapunov results is actually a well known fact also in other nonlinear control research areas.
} 
corresponding LMIs are infeasible exactly when the linear closed-loop system stops being exponentially stable (despite the fact that, based on Theorem 5.4 we know that the reset closed-loop is exponentially stable for any positive or negative $a_{c}$ ).

The most natural relaxation of the quadratic conditions of Proposition 5.1 and Corollary 5.3 consists in piecewise quadratic conditions where several quadratic functions are selected in different cones of the state space and patched together to form a unique piecewise quadratic function. The arising patched function will be continuous as long as the quadratic functions coincide at the patching surfaces and will be homogeneous of degree two because the patching surfaces are cone boundaries. In general, piecewise quadratic relaxations of convex quadratic conditions lead to non convex formulations that do not correspond to LMIs. However, in our case, it is possible to write convex conditions by exploiting the special structure of the flow and jump sets. The corresponding result is reported next.

Theorem 5.5. (Piecewise quadratic Lyapunov conditions) Consider the reset control system (5.16), 5.17) with the matrix selection (5.18). Assume (without loss of generality) that the plant (5.15) is in observability canonical form (so that $C_{p}=\left[\begin{array}{llll}0 & \cdots & 0 & 1\end{array}\right]$ ). Select either $w=r$ and $B=B_{r}$ or $w=d$ and $B=B_{d}$. Choose any $N \geq 2$ and any $\theta_{\epsilon}>0$. Define $\theta_{i}, i=0, \ldots, N$ such that $-\theta_{\epsilon}=\theta_{0}<0<\theta_{1}<\cdots<\frac{\pi}{2}<$ $\theta_{N}=\frac{\pi}{2}+\theta_{\epsilon}$ (for example, in our case studies we select $\theta_{i}=\frac{i}{N} \frac{\pi}{2}$, for all $i \in\{1, \ldots, N-1\})$. Define the angle vectors $\Theta_{i} \in \mathbb{R}^{n}$ as

$$
\Theta_{i}=\left[\begin{array}{lll}
01 \times n-2 & \sin \left(\theta_{i}\right) & \cos \left(\theta_{i}\right)
\end{array}\right]^{\top}, \quad i=0, \ldots, N,
$$

and their orthogonal complements $\Theta_{i \perp}$ (so that $\Theta_{i \perp}^{\top} \Theta_{i}=0$ ) as

$$
\Theta_{i \perp}:=\left[\begin{array}{ccc}
I & 0 & 0 \\
0 & \cos \left(\theta_{i}\right) & -\sin \left(\theta_{i}\right)
\end{array}\right]^{\top}, \quad i=0, \ldots, N .
$$

Define also the sector matrices $S_{i}=S_{i}^{\top} \in \mathbb{R}^{n \times n}$ as

$$
\begin{aligned}
S_{0} & :=\Theta_{0} \Theta_{N}^{\top}+\Theta_{N} \Theta_{0}^{\top} \\
S_{i} & :=-\left(\Theta_{i} \Theta_{i-1}^{\top}+\Theta_{i-1} \Theta_{i}^{\top}\right), \quad i=1, \ldots, N,
\end{aligned}
$$




$$
\begin{aligned}
S_{\epsilon 1}:= & {\left[\begin{array}{ccc}
0_{(n-2) \times(n-2)} & 0 & 0 \\
0 & 0 & \sin \left(\theta_{\epsilon}\right) \\
0 & \sin \left(\theta_{\epsilon}\right) & -2 \cos \left(\theta_{\epsilon}\right)
\end{array}\right], } \\
S_{\epsilon 2}:= & {\left[\begin{array}{ccc}
0_{(n-2) \times(n-2)} & 0 & 0 \\
0 & -2 \cos \left(\theta_{\epsilon}\right) & \sin \left(\theta_{\epsilon}\right) \\
0 & \sin \left(\theta_{\epsilon}\right) & 0
\end{array}\right] . }
\end{aligned}
$$

Define $Z=\left[\begin{array}{ll}I_{n-2} & 0_{(n-2) \times 2}\end{array}\right]$. If the following linear matrix inequalities in the variables $P_{i}=P_{i}^{\top}>0, \tau_{F i} \geq 0, i=1, \ldots, N, P_{0}=P_{0}^{\top}>0$, $\tau_{J}, \tau_{\epsilon 1}, \tau_{\epsilon 2} \geq 0, \gamma>0$ are feasible:

$$
\begin{aligned}
& {\left[\begin{array}{ccc}
A^{\top} P_{i}+P_{i} A+\tau_{F i} S_{i} & P_{i} B & C_{z}^{\top} \\
\star & -\gamma I & 0 \\
\star & \star & -\gamma I
\end{array}\right]<0, i=1, \ldots, N,} \\
& {\left[\begin{array}{ccc}
Z\left(A^{\top} P_{0}+P_{0} A\right) Z^{\top} & Z P_{0} B & Z C_{z}^{\top} \\
\star & -\gamma I & 0 \\
\star & \star & -\gamma I
\end{array}\right]<0, i=1, \ldots, N \text {, }} \\
& G^{\top} P_{1} G-P_{0}+\tau_{J} S_{0} \leq 0 \\
& G^{\top} P_{1} G-P_{1}+\tau_{\epsilon 1} S_{\epsilon 1} \leq 0 \\
& G^{\top} P_{1} G-P_{N}+\tau_{\epsilon 2} S_{\epsilon 2} \leq 0 \\
& \Theta_{i \perp}^{\top}\left(P_{i}-P_{i+1}\right) \Theta_{i \perp}=0, i=0, \ldots, N-1 \\
& \Theta_{N \perp}^{\top}\left(P_{N}-P_{0}\right) \Theta_{N \perp}=0
\end{aligned}
$$

then there exists a small enough $\bar{\rho}>0$ such that for any fixed $\rho \in(0, \bar{\rho})$, the FORE control system (5.16), (5.17) is exponentially stable and has a finite $t-\mathcal{L}_{2}$ gain from $w$ to $y$ which is smaller than $\gamma$.

Remark 5.9. (Interpretation of the LMIs (5.25)). The piecewise quadratic Lyapunov function arising from Theorem 5.5 is obtained by patching together $N$ quadratic functions (characterized by the matrices $\left.P_{1}, \ldots, P_{N}\right)$ defined in the (inflated) flow set and one quadratic function (characterized by the matrix $P_{0}$ ) in the jump set, as represented in Figure 5.5. Note that in most of the state space either a flow or a jump condition needs to be satisfied, except for the dashed sectors in the figure, where both the jump and flow conditions are enforced by 


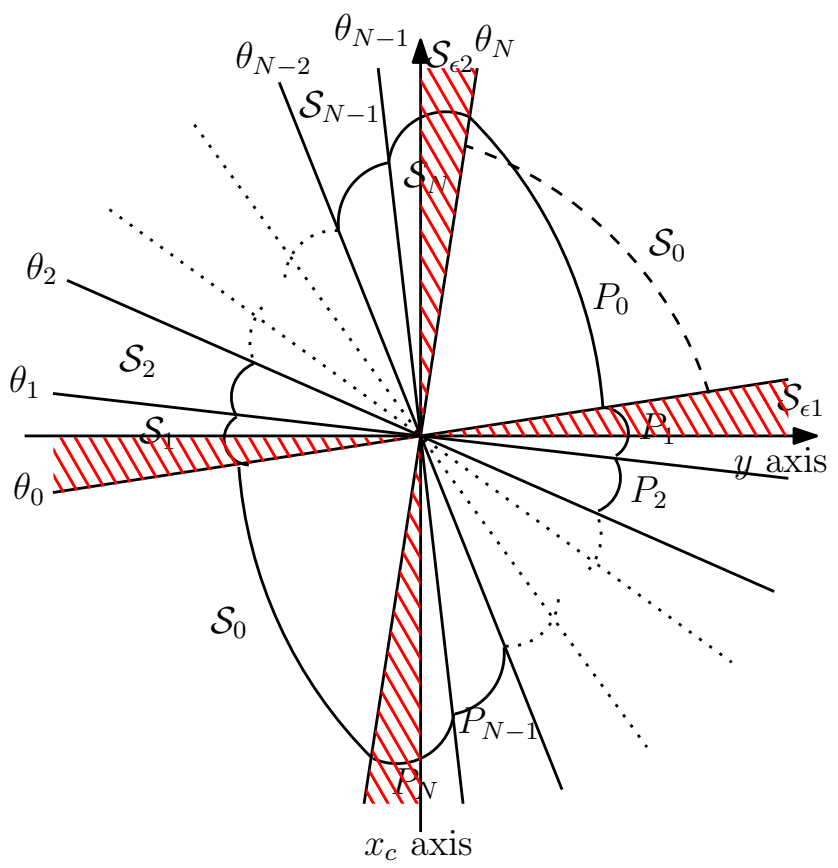

Figure 5.5: The type of piecewise quadratic Lyapunov functions constructed in Theorem 5.5

(5.25). The level set sketched in Figure 5.5 represents a possible solution arising from the LMI constraints. In particular, conditions (5.25f), $5.25 \mathrm{~g}$ ensure that the Lyapunov function is continuous on the patching surfaces. Moreover, condition 5.25c enforces the jump condition from the set $\mathcal{S}_{0}$, condition $(5.25 \mathrm{~d})$ enforces it from the set $\mathcal{S}_{\epsilon 1}$ corresponding to the portion of the set $\mathcal{S}_{1}$ overlapping with the jump set, and similarly for condition 5.25e and $\mathcal{S}_{N}$. Finally, conditions 5.25a ensure that the proposed Lyapunov function is a disturbance attenuation Lyapunov function everywhere except for $\mathcal{S}_{0}$.

Remark 5.10. Note that given any solution to (5.25), all the variables can be scaled by an arbitrary constant while preserving its feasibility. It is therefore useful to also impose $P_{i}>I, i=0, \ldots, N$ (without loss of generality) to obtain reasonable values in the entries of the matrices $P_{i}$. Moreover, these constraints are useful for implementation purposes, 
where the LMI constraints (5.25) can be solved by solving an auxiliary LMI problem consisting of only strict linear matrix inequalities.

In particular, once a very small tolerance $\varepsilon$ has been fixed, the non strict LMIs (5.25c), 5.25e can be replaced by the strict LMIs

$$
\begin{aligned}
G^{\top} P_{1} G-P_{0}+\tau_{J} S_{0} & <0 \\
G^{\top} P_{1} G-P_{N}+\tau_{\epsilon 2} S_{\epsilon 2} & <0
\end{aligned}
$$

while the nonstrict LMI (5.25d) should be broken in the following two conditions

$$
\begin{gathered}
-\left[\begin{array}{cc}
0_{1 \times(n-1)} & 1
\end{array}\right] P_{1}\left[\begin{array}{c}
0_{(n-2) \times 1} \\
1 \\
0
\end{array}\right]+\tau_{\epsilon 1} \sin \left(\theta_{\epsilon}\right)<0 \\
{\left[\begin{array}{ccc}
-\varepsilon I & \left.\left[\begin{array}{cc}
I_{n-2} & 0_{(n-2) \times 2}
\end{array}\right] P_{1}\left[\begin{array}{c}
0_{(n-1) \times 1} \\
1
\end{array}\right]\right]<0 . \\
\star & -\varepsilon I
\end{array}\right]}
\end{gathered}
$$

Finally, the equality constraints $(5.25 f)$ can be replaced by the LMIs

$$
\left[\begin{array}{cc}
-\varepsilon I & \Theta_{i \perp}^{\top}\left(P_{i}-P_{i+1}\right) \Theta_{i \perp} \\
\star & -\varepsilon I
\end{array}\right]<0, \quad i=0, \ldots, N-1,
$$

(and similarly for $(5.25 \mathrm{~g})$ ). The arising solutions will satisfy the LMIs (5.25) up to a very small tolerance (proportional to the size of $\varepsilon$ ). $\lrcorner$

\subsection{Numerical examples}

In this section we show how the LMI formulations given in Section 5.4 can be used to establish useful stability and performance properties of reset control systems involving FOREs. We first address the most classical example of a Clegg integrator (namely a FORE with $a_{c}=$ 0 ) connected to an integrator, then we address the case of a FORE connected to an integrator, which has been studied in Section 5.4 .2 by way of analytic Lyapunov constructions and finally we discuss a higher order example.

Example 5.1. (A Clegg integrator controlling an integrator plant) One of the simplest reset systems considered in the literature corresponds 
to a Clegg integrator connected in feedback with an integrating plant. Studying the stability of this simple closed-loop by Lyapunov tools is already a challenging task to accomplish which was addressed and solved in Hollot et al. [1997, Hu et al. [1997]. The equations of the closed-loop system before temporal regularization can be written as

$$
\left\{\begin{array}{cc}
\dot{y}=x_{c}+d, & \text { if } x_{c}(r-y) \geq 0, \\
\dot{x}_{c}=r-y, & \text { if } x_{c}(r-y) \leq 0 . \\
x_{c}^{+}=0, &
\end{array}\right.
$$

Exponential and $t-\mathcal{L}_{2}$ stability of this closed-loop is established in Theorem 5.4 in Section 5.4.2. Here, by employing the LMI-based techniques of Section 5.4.3, we also give a tight estimate on the $t-\mathcal{L}_{2}$ gain of the system from the input $d$ to the output $y$. In light of the stability properties established in Theorem 5.4 and e.g., in Hollot et al. [1997, $\mathrm{Hu}$ et al. [1997, we may expect to get an estimate of the $t-\mathcal{L}_{2}$ gain from $d$ to $y$ using the quadratic Lyapunov functions proposed in Proposition 5.1. However, the LMI constraints therein proposed turn out to be non feasible for this particular problem. Indeed, as discussed in Section 5.4.3, even for such a simple closed-loop system, a piecewise quadratic Lyapunov function is necessary to obtain an estimate of the $t-\mathcal{L}_{2}$ gain. When using the LMIs of Theorem 5.5 it is necessary to use at least $N=2$ to prove the closed-loop exponential stability ${ }^{2}$. Moreover, as $N$ increases, tighter and tighter bounds are obtained for the $t-\mathcal{L}_{2}$ gain of the system. Table 5.1 reports some of the values obtained by increasing the number of regions.

\begin{tabular}{|c||c|c|c|c|c|c|}
\hline$N$ & 2 & 3 & 4 & 8 & 15 & 50 \\
\hline \hline gain & 2.8338 & 1.8188 & 1.3766 & 0.9145 & 0.8839 & 0.8701 \\
\hline
\end{tabular}

Table 5.1: Example 5.1 estimates of the $t-\mathcal{L}_{2}$ gain of 5.29 determined by piecewise quadratic Lyapunov functions.

It is instructive to study the level sets of the piecewise quadratic Lyapunov functions arising from the LMIs of Theorem 5.5. For the

\footnotetext{
${ }^{2}$ All the numbers in Table 5.1 have been determined following the strategy commented in Remark 5.10 with $\varepsilon=1 e-10$.
} 

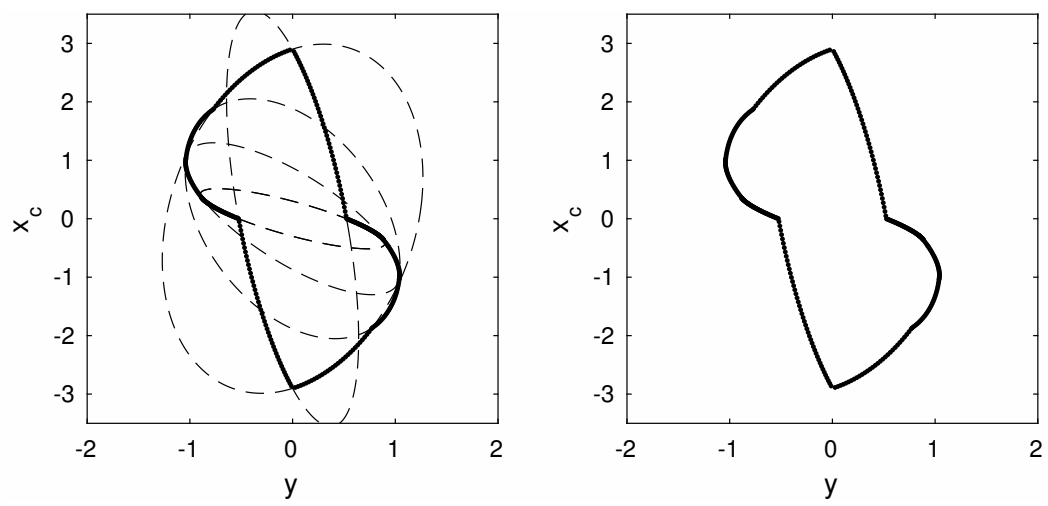

Figure 5.6: Example 5.1 level sets of the five quadratic Lyapunov functions used for the case $N=4$ (left). Level set of the arising piecewise quadratic Lyapunov function (right).

case of $N=4$ (corresponding to five quadratic functions), Figure 5.6 shows on the left the level sets of the quadratic functions involved in the piecewise quadratic construction, and on the right, a level set of the patched piecewise quadratic Lyapunov function. It is interesting to notice that the Lyapunov function is non-convex. Qualitatively, the nonconvexity should allow for significant degrees of freedom in Lyapunov functions constructions. Indeed, it has been recently shown in Blanchini and Savorgnan 2006 that there are situations where convex Lyapunov functions are insufficient to prove stability.

Example 5.2. (A FORE controlling an integrator) As discussed in Chapter 2 , reset control systems can overcome certain limitations of linear control systems. In particular, for an integrating plant, it is shown in Hollot et al. 2001] that the reset controller can achieve arbitrarily large rising time of the closed loop while guaranteeing zero overshoot. This is proved to be non achievable for linear control systems (see Hollot et al. 2001] for details). In [Hollot et al. 2001, §9.2.1], a FORE with time constant $a_{c}=1$ connected in unit negative feedback to an integrator is used to illustrate this fact. The closed-loop responses of the system without reset (solid) and of the system with resets (bold) is shown in Figure 5.4 reported in Section 5.4 .2 when illustrating the re- 
sults of Theorem 5.4. According to our notation, the closed-loop system before temporal regularization is described by the following dynamics

$$
\begin{cases}\dot{y}=x_{c}+d & x_{c}(r-y) \geq 0, \\ \dot{x}_{c}=a_{c} x_{c}-(r-y), & x_{c}(r-y) \leq 0, \\ x_{c}^{+}=0, & \end{cases}
$$

and corresponds to the block diagram of Figure 5.2 with $\mathcal{P}=\frac{1}{s}$ and $a_{c}=1$ in the FORE.

As for the previous example, the stability of this closed-loop system is established by Theorem 5.4 in Section 5.4.2. However, we can use the tools introduced in Sections 5.3 and 5.4 .3 for the construction of quadratic or piecewise quadratic Lyapunov functions to obtain a (tight) estimate of the input/output gain from the reference signal $r$ to the output $y$. For this example, it is of interest to compare the $t-\mathcal{L}_{2}$ gain of the reset control system to the $t-\mathcal{L}_{2}$ gain characterizing the closed loop without resets. In particular, the $t-\mathcal{L}_{2}$ gain of the linear closed loop is 1.468 and the estimate arising from the quadratic Lyapunov construction of Corollary 5.3 , corresponding to 1.84 , is not good enough to show that the $t-\mathcal{L}_{2}$ gain of the reset control system is improved as compared to the linear case. However, determining a bound using the piecewise quadratic construction of Theorem 5.5 leads to a less conservative estimate of 1.18 (determined using 51 quadratic Lyapunov functions), which is able to predict the improved performance of the reset control system.

Figure 5.7 shows on the left the level sets of the quadratic functions involved in the piecewise quadratic construction, and on the right, a level set of the patched piecewise quadratic Lyapunov function (bold) compared to the level set of the quadratic Lyapunov function establishing the 1.84 gain estimate. Note that, for this example, the optimal piecewise quadratic Lyapunov function is nonconvex again.

It is useful to emphasize that it not necessary for the FORE element in (5.30) to be exponentially stable. Indeed, as already proved in Theorem 5.4, any real selection of $a_{c}$ enforces closed-loop stability and finite $t-\mathcal{L}_{2}$ gain from $d$ to $y$. Figure 5.8 represents the different bounds obtained by the piecewise quadratic construction of Theorem 5.5 for a 

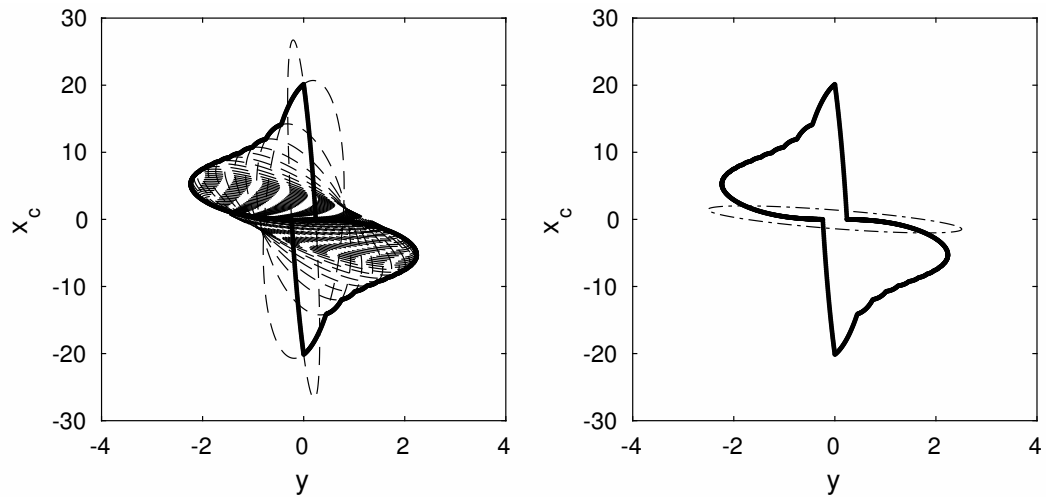

Figure 5.7: Example 5.2 Left: level sets of the 51 quadratic Lyapunov functions used for the case $N=50$. Right: level set of the arising piecewise quadratic Lyapunov function (bold) and of the quadratic Lyapunov function from Corollary 5.3 .

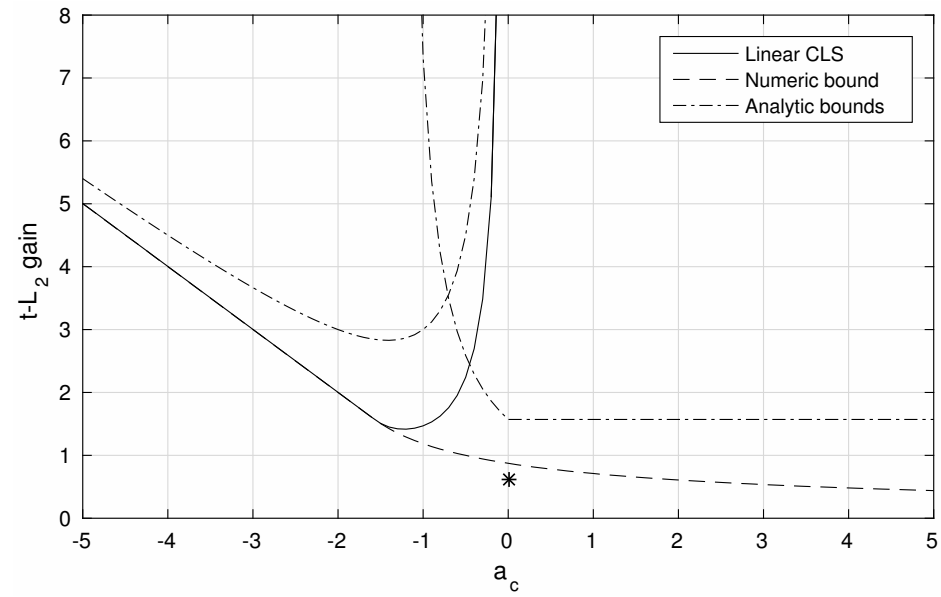

Figure 5.8: Example 5.2. The $t-\mathcal{L}_{2}$ gain estimates obtained by using PWQ Lyapunov functions (dashed) for different values of the FORE's pole $a_{c}$, compared to the corresponding linear performance (solid) and to the analytic bounds established in Theorem 5.4 (dash-dotted). The "*" at $a_{c}=0$ corresponds to the lower bound established in Remark 5.7

wide range of values of $a_{c}$. This gain curve (solid) is compared to the corresponding linear closed-loop gain (dashed), namely the gain that 
one obtains from the linear closed-loop in the absence of resets. Inevitably, as $a_{c}$ approaches zero from the left, this curve goes to infinity because the linear system is exponentially unstable for positive $a_{c}$ 's. The bounds on the $t-\mathcal{L}_{2}$ gain established in Theorem 5.4 by way of analytic Lyapunov constructions are also reported in Figure 5.8 (dashdotted curves). Note that the numerical estimates arising from Theorem 5.5 improve upon the analytic bounds.

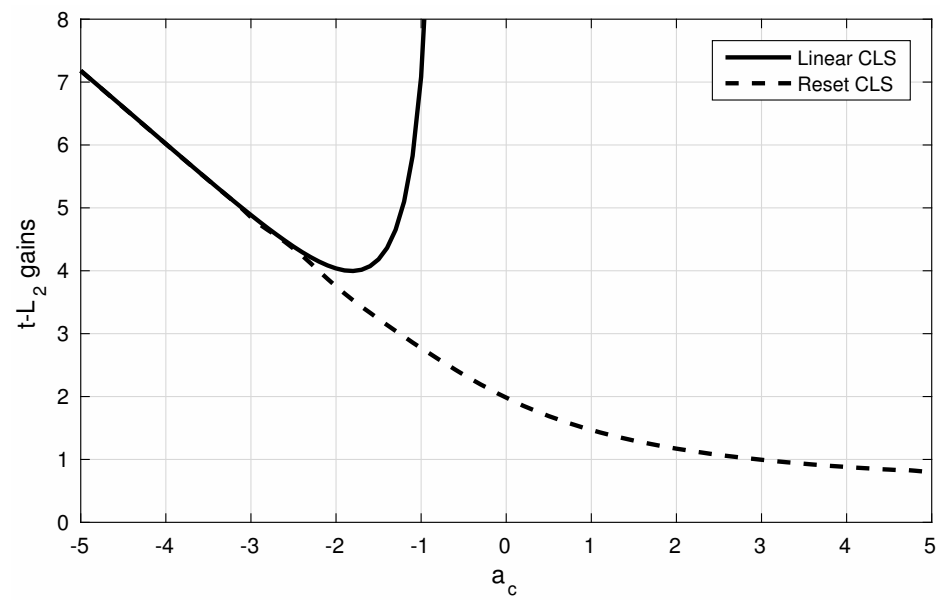

Figure 5.9: $t-\mathcal{L}_{2}$ gains of linear and reset closed loops for Example 5.3 , as a function of the pole of the FORE.

Example 5.3. As a last example we consider a two dimensional linear plant controlled by a FORE discussed in Nešić et al. [2005] and previously introduced in Hollot et al. 2001. In this example, a FORE element whose linear part is characterized by the transfer function $\frac{1}{s+1}$ (namely with $a_{c}=-1$ ) controls via a negative unitary feedback a SISO plant whose transfer function is $\mathcal{P}=\frac{s+1}{s(s+0.2)}$. For this example, the control system involving the FORE is shown in Hollot et al. 2001] to behave more desirably than the linear control system. It was shown in Hollot et al. 2001] that the reset system had only about $40 \%$ overshoot of the linear closed-loop system while retaining the rise time of the linear design. This example can be further interpreted using our 
results. Indeed, when computing the $t-\mathcal{L}_{2}$ gain from the plant input to the plant output, the linear closed-loop system has an $\mathcal{H}_{\infty}$ norm around 5 , while using the piecewise quadratic construction of Theorem 5.5 we obtain that the $t-\mathcal{L}_{2}$ gain of the reset system is smaller than 2.77 .
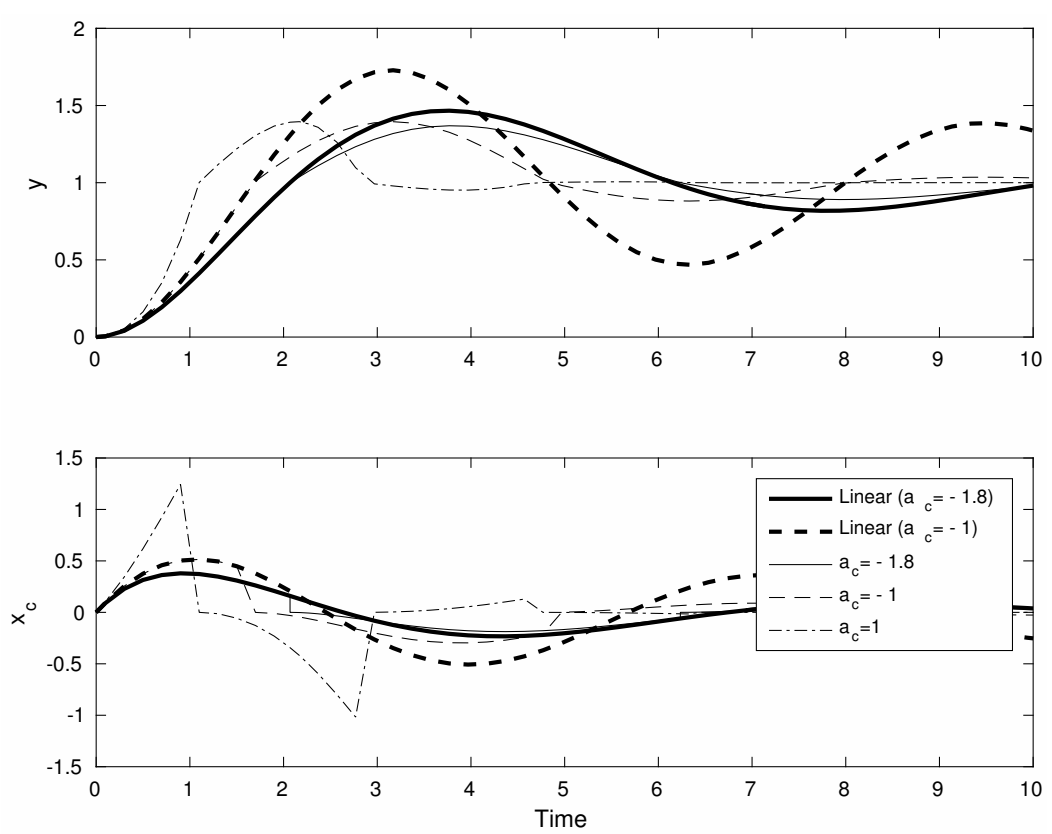

Figure 5.10: Time responses for different values of the FORE pole, with and without resets for Example 5.3

Figure 5.9 reports the $t-\mathcal{L}_{2}$ gains for the linear closed-loop and the reset closed-loop as a function of the pole of the FORE $(N=10$ is chosen in Theorem 5.5). Similar to the previous example, for positive values of $a_{c}$ (unstable FOREs) the linear closed-loop is unstable, while the reset closed loop induces improved gains. The case studied in Hollot et al. 2001] corresponds to the horizontal coordinate $a_{c}=-1$ in Figure 5.9 .

For this example it seems appropriate to show the different responses obtained when using the linear controller guaranteeing the minimum $\mathcal{H}_{\infty}$ norm in Figure 5.9 (namely $a_{c} \approx-1.8$ ) and when using 
the FORE for different values of $a_{c}$. These responses are reported in Figure 5.10 . 
6

Towards reset $\mathcal{H}_{\infty}$ control design

\subsection{Overview}

In this chapter, we extend, in a first stage, the results of Chapter 5 (see also Nešić et al. 2008b]), providing relaxed Lyapunov-based conditions to estimate an $\mathcal{L}_{2}$ gain bound for a class of hybrid control systems. That includes several works in the literature (notably Zaccarian et al. 2005, 2011], Nešić et al. 2011, Fichera et al. 2012b, 2013]). Convex conditions for simultaneous design of an optimal multi-objective $\mathcal{H}_{\infty}$ reset controller minimizing the decay rate and minimizing the $\mathcal{L}_{2}$ gain for a linear continuous-time plant are proposed. The reset controller architecture in Fichera et al. 2012b] and the analysis results are combined by means of a suitable change of coordinates, in order to obtain convex synthesis conditions. A preliminary version of the construction proposed here had been reported in Fichera et al. 2012c and is not discussed here. As compared to Fichera et al. [2012c] the hybrid controller architecture that we use here allows obtaining an $\mathcal{H}_{\infty}$ reset controller whose flow map is not necessarily stabilizing in the whole state space (see also Zaccarian et al. 2011) thereby possibly leading to more aggressive actions during flowing solutions. Satoh [2011] and Yuan and $\mathrm{Wu} 2014$ also provide conditions to design a complete $\mathcal{H}_{\infty}$ reset con- 
troller. The proposed techniques are based on different structures in the Lyapunov matrix $P$ certifying the quadratic stability of the hybrid closed loop system.

\subsection{Full state availability}

In this first part, we consider a controller architecture $\mathcal{H}_{c}$ in which the reset law realized (namely, the right hand side of the $x_{c}^{+}$equation) requires the availability of the full state $x_{p}$. This is a strong assumption that will be removed in the subsequent section by the addition of a Luenberger observer.

\subsubsection{Problem statement}

Let us consider Figure 6.1, which describes the system under consideration. Hence, according to Figure 6.1, consider a linear continuous-time

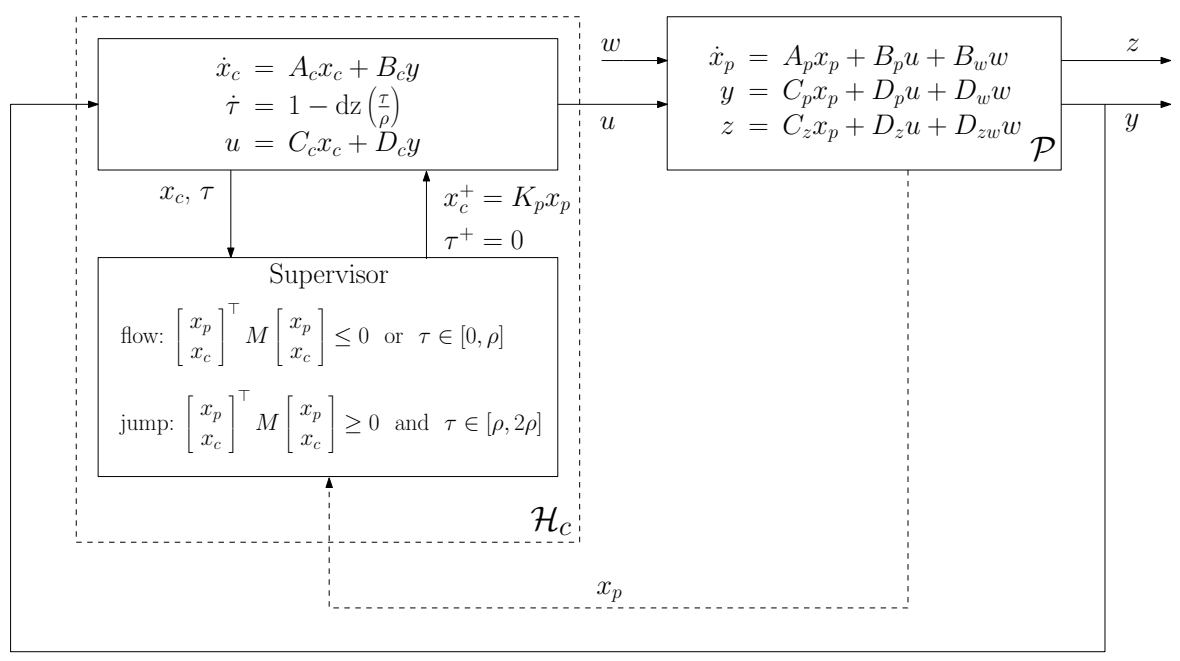

Figure 6.1: Block diagram of the proposed reset controller.

plant $\mathcal{P}$, represented by

$$
\left\{\begin{aligned}
\dot{x}_{p} & =A_{p} x_{p}+B_{p u} u+B_{p w} w \\
z & =C_{z} x_{p}+D_{z u} u+D_{z w} w \\
y & =C_{p} x_{p}+D_{p u} u+D_{p w} w
\end{aligned}\right.
$$


where $x_{p} \in \mathbb{R}^{n_{p}}$ is the state of the plant, $u \in \mathbb{R}^{n_{u}}$ is the control input, $y \in \mathbb{R}^{n_{y}}$ is the measured output (used for the feedback), $w \in \mathbb{R}^{n_{w}}$ is an exogenous input (comprising disturbances and references) and $z \in \mathbb{R}^{n_{z}}$ is the performance output. It is the same model as in Chapter 5 by considering the presence of an exogenous output (compare (5.1) with (6.1).

To keep the presentation simple, algebraic loops are avoided by making the following typical assumption.

Assumption 6.1. Plant (6.1) is strictly proper from $u$ to $y$, namely $D_{p u}=0$.

Note that the previous assumption is not very restrictive. Indeed, if in plant $\mathcal{P} D_{p u} \neq 0$, then one can always define $\bar{y}:=y-D_{p u} u$ and use $\bar{y}$ as a new plant measurement output.

In the following, the objective is to propose an optimization-based synthesis method for simultaneous design of flow map, jump map, flow set and jump set of a plant-order reset controller. The results of the previous chapter are used and, via a change of coordinates similar to Fichera et al. 2012c], a convex LMI formulation with a line search are obtained. The proposed architecture can be well interpreted as a reset version of continuous-time $\mathcal{H}_{\infty}$ controller synthesis.

The first reset controller architecture $\mathcal{H}_{c}$ that we propose is given by

$$
\begin{aligned}
& \left\{\begin{array}{rl}
\dot{x}_{c} & =A_{c} x_{c}+B_{c} y \\
\dot{\tau} & =1-\mathrm{dz}\left(\frac{\tau}{\rho}\right)
\end{array}, \quad(x, \tau) \in \mathcal{C},\right. \\
& \left\{\begin{array}{l}
x_{c}^{+}=K_{p} x_{p} \\
\tau^{+}=0
\end{array}, \quad(x, \tau) \in \mathcal{D},\right. \\
& u=C_{c} x_{c}+D_{c} y,
\end{aligned}
$$

where $x=\left(x_{p}, x_{c}\right), x_{c} \in \mathbb{R}^{n_{c}}$ and $\tau \in[0,2 \rho]$ is the dwell-time logic timer. Furthermore, the flow and jump sets $\mathcal{C}$ and $\mathcal{D}$ are defined similarly to $5.2 \mathrm{a})-(5.2 \mathrm{~d})$ as follows:

$$
\mathcal{C}:=\{(x, \tau): x \in \mathcal{F} \text { or } \tau \in[0, \rho]\}
$$




$$
\begin{aligned}
& =\{(x, \tau): x \in \mathcal{F}\} \cup\{(x, \tau): \tau \in[0, \rho]\}, \\
\mathcal{D} & :=\{(x, \tau): x \in \mathcal{J} \text { and } \tau \in[\rho, 2 \rho]\} \\
& =\{(x, \tau): x \in \mathcal{J}\} \cap\{(x, \tau): \tau \in[\rho, 2 \rho]\},
\end{aligned}
$$

with $\mathcal{F}$ and $\mathcal{J}$ symmetric cones defined by a matrix $M=M^{\top} \in \mathbb{R}^{n \times n}$, with $n=n_{p}+n_{c}$, as

$$
\begin{aligned}
\mathcal{F} & :=\left\{x \in \mathbb{R}^{n}: x^{\top} M x \leq 0\right\} \\
\mathcal{J} & :=\left\{x \in \mathbb{R}^{n}: x^{\top} M x \geq 0\right\}
\end{aligned}
$$

In the previous equations, $M$ is a design parameter defined as

$$
M:=\operatorname{He}\left(P A+\frac{\tilde{\alpha}}{2} P\right)
$$

with $A$ representing the flow map of the closed-loop system (see 6.1) and also (6.3) below) and $\tilde{\alpha}, P$ being controller parameters to be defined. Hybrid controller 6.2 is the same as the one in [Fichera et al. 2012b, Theorem 1], with differences clarified below in Remark 6.1.

Similar to Fichera et al. 2012c], Satoh [2011], Prieur et al. 2010, 2011, 2013, we consider that state feedback reset laws, namely the jump map and sets $\mathcal{C}$ and $\mathcal{D}$ in $(6.2)$, depend on the knowledge of the plant state $x_{p}$ at jump times, which is a strong assumption. Nevertheless, applying the results in Fichera et al. 2013, whenever the plant state is detectable from $y$, we may implement the proposed controller in output feedback from $y$ and without a direct measurement of $x_{p}$, preserving the closed-loop exponential stability properties established by this design.

The feedback interconnection between $\mathcal{H}_{c}$ and $\mathcal{P}$ is always possible since Assumption 6.1 implies well-posedness in the linear sense. Thus, we obtain the hybrid closed-loop system $(6.1)-(6.2)$ with $x=$ 
$\left[x_{p}^{\top} x_{c}^{\top}\right]^{\top} \in \mathbb{R}^{n}$, with the selection:

$$
\begin{aligned}
\left(\begin{array}{c|c}
A & B \\
\hline G & - \\
\hline- & M \\
\hline \bar{C}_{z} & \bar{D}_{z w} \\
\hline \bar{C}_{p} & \bar{D}_{p w}
\end{array}\right)=\left(\begin{array}{cc|c}
\bar{A}_{p} & \bar{B}_{p} & \bar{B}_{p w} \\
\bar{B}_{c} & \bar{A}_{c} & \bar{B}_{c w} \\
\hline G & - \\
\hline- & M \\
\hline \bar{C}_{z} & \bar{D}_{z w} \\
\hline \bar{C}_{p} & \bar{D}_{p w}
\end{array}\right) \\
=\left(\begin{array}{cc|c}
A_{p}+B_{p u} D_{c} C_{p} & B_{p u} C_{c} & B_{p w}+B_{p u} D_{c} D_{p w} \\
B_{c} C_{p} & A_{c} & B_{c} D_{p w} \\
\hline I & 0 & - \\
K_{p} & 0 & - \\
\hline- & - & \operatorname{He}\left(P A+\frac{\alpha}{2} P\right) \\
\hline C_{z}+D_{z u} D_{c} C_{p} & D_{z u} C_{c} & D_{z w}+D_{z u} D_{c} D_{p w} \\
\hline C_{p} & 0 & D_{p w}
\end{array}\right) .
\end{aligned}
$$

In the sequel, we refer to the interconnection between $\mathcal{H}_{c}$ and $\mathcal{P}$ as 6.1)- 6.2), 6.3).

Remark 6.1. As compared to Fichera et al. 2012b], here we want to use the same reset controller architecture to propose a multi-objective simultaneous synthesis optimizing the $t$-decay rate and the $t$ - $\mathcal{L}_{2}$ performance introduced in Definition 5.1. Note that in Fichera et al. 2012b], the proposed optimization-based synthesis for overshoot reduction only concerned the design of the reset loop. In other words for any given flow map (namely, matrix $A$ is given), a solution was proposed to design flow and jump sets and the jump map (namely, $M$ and $K_{p}$ in 6.2) to achieve global exponential stability of the origin, guaranteeing overshoot reduction. However, controller matrices $\left(A_{c}, B_{c}, C_{c}, D_{c}\right)$ were not part of the design. Here instead, similar to Fichera et al. [2012c, Satoh 2011, we propose an optimization-based synthesis to completely design the $\mathcal{H}_{\infty}$ reset controller, that is, flow and jump maps and flow and jump sets altogether. 


\subsection{2 $\mathcal{H}_{\infty}$ reset controller design}

The following theorem states sufficient conditions for an optimizationbased design of the $\mathcal{H}_{\infty}$ reset controller $(6.2)$ with respect to the $t$-decay rate $\tilde{\alpha}$ and the $t-\mathcal{L}_{2}$ gain $\gamma$, introduced in Definition 5.1. In particular, the theorem provides an almost convex procedure to design a plantorder $\mathcal{H}_{\infty}$ reset controller. The result is proved by merging the exponential stability results in Fichera et al. [2012b], from which a $t$-decay rate can be inferred, and the $t-\mathcal{L}_{2}$ analysis in Proposition 5.1. In particular, the synthesis is performed without requiring growth at jumps of the Lyapunov function. Thus if $\rho=0$, the set of allowable values of $\rho$ is always non empty and (5.11) is equivalent to 6.6 in this particular case. The details of the proof are reported in Fichera et al. [2016].

Theorem 6.1. Given plant 6.1 satisfying Assumption 6.1 and any set of positive definite matrices $Y=Y^{\top} \in \mathbb{R}^{n_{p} \times n_{p}}, W=W^{\top} \in \mathbb{R}^{n_{p} \times n_{p}}$, matrices $\hat{A} \in \mathbb{R}^{n_{p} \times n_{p}}, \hat{B} \in \mathbb{R}^{n_{p} \times n_{y}}, \hat{C} \in \mathbb{R}^{n_{u} \times n_{p}}, \hat{D} \in \mathbb{R}^{n_{u} \times n_{y}}$, positive scalars $\bar{\gamma}, \alpha$ and a nonnegative scalar $\tau_{S} \geq 0$ satisfying

$$
\begin{aligned}
& {\left[\begin{array}{cc}
Y & I \\
I & W
\end{array}\right]>0,} \\
& \operatorname{He}\left(A_{p} Y+B_{p u} \hat{C}\right)+\alpha Y<0, \\
& \operatorname{He}\left(\left[\begin{array}{cc}
M_{1} & M_{2} \\
M_{3} & M_{4}
\end{array}\right]\right)<0 .
\end{aligned}
$$

with

$$
\begin{array}{rl}
M_{1} & =\left(1-\tau_{S}\right)\left[\begin{array}{cc}
A_{p} Y+B_{p u} \hat{C} & A_{p}+B_{p u} \hat{D} C_{p} \\
\hat{A} & W A_{p}+\hat{B} C_{p}
\end{array}\right]-\frac{\tau_{S} \tilde{\alpha}}{2}\left[\begin{array}{cc}
Y & I \\
I & W
\end{array}\right] \\
M_{2} & =\left[\begin{array}{cc}
B_{p w}+B_{p u} \hat{D} D_{p w} & 0 \\
W B_{p w}+\hat{B} D_{p w} & 0
\end{array}\right] \\
0 & 0 \\
M_{3} & =\left[\begin{array}{cc}
C_{z} Y+D_{z u} \hat{C} & C_{z}+D_{z u} \hat{D} C_{p}
\end{array}\right] \\
-\frac{\bar{\gamma}}{2} I & 0 \\
M_{4} & =\left[\begin{array}{cc}
D_{z w}+D_{z u} \hat{D} D_{p w} & -\frac{\bar{\gamma}}{2} I
\end{array}\right]
\end{array}
$$


for some $\tilde{\alpha} \in(0, \alpha]$, select the controller parameters as:

$$
\begin{aligned}
& P=\left[\begin{array}{cc}
W & -W \\
-W & W+\left(Y-W^{-1}\right)^{-1}
\end{array}\right], \\
& K_{p}=\left(Y-W^{-1}\right) Y^{-1}, \\
& D_{c}=\hat{D}, \\
& C_{c}=\left(\hat{C}-D_{c} C_{p} Y\right)\left(Y-W^{-1}\right)^{-1}, \\
& B_{c}=-W^{-1} \hat{B}+B_{p u} D_{c}, \\
& A_{c}=-W^{-1}\left(\hat{A}+W B_{c} C_{p} Y-W B_{p u} C_{c}\left(Y-W^{-1}\right)\right. \\
&\left.\quad \quad-W\left(A_{p}+B_{p u} D_{c} C_{p}\right) Y\right)\left(Y-W^{-1}\right)^{-1} .
\end{aligned}
$$

Then, there exists $\bar{\rho}>0$ such that for any $\rho \in(0, \bar{\rho})$ :

- $t$-decay rate: the set $\mathcal{A}$ in $(5.3)$ is globally exponentially stable for the hybrid closed-loop system (6.1)- 6.2), (6.3), with $w=0$, and the $t$-decay rate is $\tilde{\alpha} / 2$;

- $\mathcal{H}_{\infty}$ specification: for any $w \in t-\mathcal{L}_{2}$, the $t$ - $\mathcal{L}_{2}$ gain from $w$ to $z$ is smaller than or equal to

$$
\gamma=\min \left\{\bar{\gamma}, \sqrt{2}\left|D_{z w}\right|\right\} .
$$

Remark 6.2. Optimization issues. Theorem 6.1 gives an LMI-based convex procedure with a line-search on $\tau_{S} \geq 0$ to design an $\mathcal{H}_{\infty}$ reset controller. Note that the line search must be carried out to get convexity of the optimization. Indeed, (6.4) becomes an LMI after fixing $\tau_{S}$. The $(\alpha, \bar{\gamma})$ trade-off in our design can be addressed by fixing $\tilde{\alpha}=\alpha>0$ and solving an eigenvalue problem minimizing $\bar{\gamma}$. Then the $t$-decay rate is fixed to be $\tilde{\alpha} / 2$ and the $t-\mathcal{L}_{2}$ gain can be minimized. It may sometimes be desirable to pick $\tilde{\alpha}$ smaller than $\alpha$ to induce longer times between pairs of consecutive resets. Before computing $\tilde{\alpha}$ smaller than $\alpha$, it is simpler to first apply Theorem 6.1 with $\tilde{\alpha}=\alpha$, and then recompute unknown variables with $\tilde{\alpha}$ a suitable fraction $\alpha$, and so on, to find the "best" couple of variables $(\tilde{\alpha}, \alpha)$.

Remark 6.3. Line-search effects. Note that (6.4c) corresponds to (5.13a) in Proposition 5.1, and then it is clear that $\tau_{S}$ is the multiplier used in the S-procedure that allows relaxing the flow condition 
but only enforcing it in $\tilde{\mathcal{F}}_{\epsilon}$ (see also $(5.10 \mathrm{~b})$ ). In particular whenever $\tau_{S}=0$, the flow set does not appear in $(6.4 \mathrm{c})$ so that, according to Remark 5.4, item 1, condition (5.11) (and so the second term in (6.6p) is not needed, and the $t-\mathcal{L}_{2}$ gain is $\gamma=\bar{\gamma}$. This is the approach that was followed in the preliminary work of Fichera et al. 2012c. The choice of $\tau_{S}=0$ is, however, conservative because in this case $(6.4 \mathrm{c})$ holds for all $x \in \mathbb{R}^{n} \backslash\{0\}$ and therefore the Lyapunov flow condition holds in all the state space. Whenever $\tau_{S}>0,6$, 6.4c holds for all $x \in \tilde{\mathcal{F}}_{\epsilon}$, so that the fact that trajectories are forced to only flow in $\tilde{\mathcal{F}}_{\epsilon}$ is taken into account. In this latter case, condition (5.11) (and so (6.6) needs to be satisfied. Furthermore several different scenario can be characterized, according to the value of $\tau_{S}$ in $\left.6.4 \mathrm{c}\right)$ :

- $0 \leq \tau_{S}<1$ implies that $A$ in 6.3 is Hurwitz, namely the linear dynamics before resets is exponentially stable (that is, the flow map of the $\mathcal{H}_{\infty}$ reset controller stabilizes the continuous-time loop);

- $\tau_{S}=1$ implies that $A$ is not necessarily Hurwitz;

- $\tau_{S}>1$ implies that $A$ is non Hurwitz and interesting closed-loop responses exhibiting exponentially diverging branches might be observed (see Zaccarian et al. 2011]), because the linear dynamics before resets is exponentially unstable.

According to Proposition 5.1, inequalities (6.4a and $6.4 \mathrm{c}$ imply the existence of a matrix $P=\left[\begin{array}{ll}Y & Z \\ Z\end{array}\right]^{-1}=P^{\top}>0$ satisfying (5.13) and then the $t-\mathcal{L}_{2}$ result follows from Proposition 5.1. In the meantime, $6.4 \mathrm{a}$ and $6.4 \mathrm{~b}$ guarantee that the $t$-decay rate is assessed. Note that we are still using the same Lyapunov function for each objective, and the conservativeness discussed in [Scherer et al., 1997, §IV.A] still holds. However, since the controller state can be reset (this is an extra degree of freedom), better compromises can be obtained in the multi-objective context. 


\subsection{Partial state availability}

It is important to observe that an important weakness of the proposed reset $\mathcal{H}_{\infty}$ architecture 6.2 is that, despite the typical output feedback structure of the flow dynamics, both the jump/flow sets and the jump rule depend on the availability of the complete plant state $x_{p}$. Such a drawback has been considered in Chapter 4 , where two solutions for the output feedback extension, allowing to study an output feedback implementation of (6.2) under a less general structure than that of closed loop (5.1), are considered.

In the case with additive perturbation, a parallel result to that of Theorems 4.1 and 4.2 is given by the following proposition, whose proof uses a different approach from Fichera et al. [2013], more similar in nature to the results in Teel 2010.

Proposition 6.1. Consider plant (6.1) in feedback with a hybrid controller of the form (6.2), where the state $x=\left(x_{p}, \hat{x}_{p}, x_{c}\right)$ and the plant state estimate $\hat{x}_{p}$ arises from the continuous-time Luenberger observer having flow equation:

$$
\dot{\hat{x}}_{p}=\left(A_{p}-L C_{p}\right) \hat{x}_{p}+\left(B_{p u}-L D_{p u}\right) u+L y,
$$

and jump equation $\hat{x}_{p}^{+}=\hat{x}_{p}$. If matrix $A_{p}-L C_{p}$ is Hurwitz, then the corresponding output feedback closed loop guarantees global exponential stability of the attractor $\mathcal{A}_{e}:=\left\{\left(x_{p}, x_{c}, \hat{x}_{p}, \tau\right):\left(\hat{x}_{p}, x_{c}, \tau\right) \in\right.$ $\mathcal{A}$ and $\left.x_{p}-\hat{x}_{p}=0\right\}$.

At this stage, it is important to note that the result of Proposition 6.1 does not allow to carry forward the performance properties established in Theorem 6.1 from the state-feedback case to the output feedback case. Nevertheless, one can expect that similar results to those in Proposition 4.1 (see also [Fichera et al. 2013, Prop. 1 \& 2]) could be proven for our case too, thus establishing some tight relation between the solutions to the state feedback and output feedback cases. Extensions to a convex observer-free reset $\mathcal{H}_{\infty}$ full output feedback design is regarded as future work.

Remark 6.4. Another route, which is an alternative to the previous one based on the use of an observer, is to use directly the available 
signals, that is in particular the measured output. Some results have been published in order to build the flow and jump sets, together with the reset rule using only the available signals.

For example, in the disturbance-free case, in Seuret et al. 2016, the way to use reset control in event-triggering strategy purpose from an emulation point of view is studied. Furthermore, in the $\mathcal{H}_{\infty}$ performance context of this chapter, Yuan and $\mathrm{Wu}$ 2014 defines the flow and jump sets from the use of the state of the controller and the output of the plant.

\subsection{Illustrative examples}

\subsubsection{Example: DC motor}

Let us illustrate the drawbacks and the advantages of the results by dealing with a DC motor already used in Section 4.3.2. A comparison with the linear classical multi-objective case Scherer et al. [1997] is also proposed.

In order to avoid fast exponential branches that may damage the actuator or require excessive bandwidth in the control system, we exploit the advantages of the LMI formulation by adding the following constraints to the conditions of Theorem 6.1

$$
\begin{aligned}
& -2 \beta_{1} \otimes X-\operatorname{He}(A X)<0, \\
& -2 \beta_{2} \sin (\theta)\left[\begin{array}{ll}
I & 0 \\
0 & I
\end{array}\right]+\left[\begin{array}{cc}
\sin (\theta) I & \cos (\theta) I \\
-\cos (\theta) I & \sin (\theta) I
\end{array}\right] \otimes A X \\
& +\left[\begin{array}{cc}
\sin (\theta) I & -\cos (\theta) I \\
\cos (\theta) I & \sin (\theta) I
\end{array}\right] \otimes(A X)^{\top}<0,
\end{aligned}
$$

where $X$ and $A X$ are given by (see Chilali and Gahinet [1996, Scherer et al. [1997):

$$
X:=\left[\begin{array}{cc}
Y & I \\
I & W
\end{array}\right], A X:=\left[\begin{array}{cc}
A_{p} Y+B_{p u} \hat{C} & A_{p}+B_{p u} \hat{D} C_{p} \\
\hat{A} & W A_{p}+\hat{B} C_{p}
\end{array}\right],
$$

and correspond to the Lyapunov matrix and the closed-loop dynamical matrix, respectively. $\beta_{1}, \beta_{2}$ and $\theta$ are design parameters. Enforcing the 


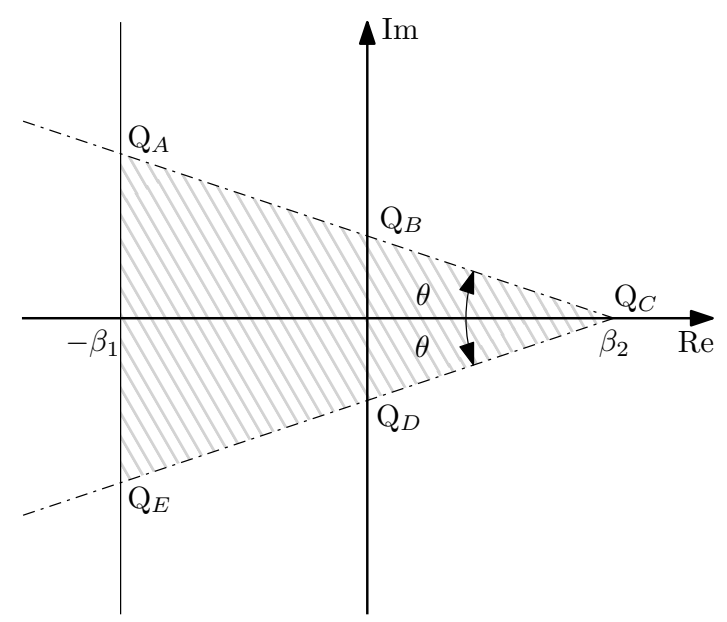

Figure 6.2: Pole placement region.

constraints in (6.8) guarantees that the poles of the closed-loop feedback (or the continuous-part of the feedback for the reset case) lay in the region pictorially shown in Figure 6.2. In particular, notice that after the linear synthesis, the poles of the closed-loop feedback will lay in the polygon $\overline{\mathrm{Q}_{A} \mathrm{Q}_{B} \mathrm{Q}_{C} \mathrm{Q}_{D} \mathrm{Q}_{E}}$. Even if exponential stability is equivalent to having the closed-loop poles in the left-side of the complex plane, the hybrid case is instead nonlinear, thus the synthesis allows for the design of an $\mathcal{H}_{\infty}$ reset controller whose continuous-time dynamics is not stabilizing. Therefore the poles of the continuous-time part of the reset closed-loop system could be placed anywhere in the complex plane, possibly generating fast positive exponential branches (while stability is induced by resets). In the sequel, we consider $\beta_{1}=50$, $\beta_{2}=25$ and $\theta=\pi / 30$ as reasonable values for the systems in exam. All the design is performed by means of YALMIP (Löfberg 2004]).

According to Fichera et al. [2013], we augment the $\mathcal{H}_{\infty}$ reset controller with an observer in order to have a complete output feedback (where also the resets depend on an estimate of the plant state provided by the observer) and we will use the $t-\mathcal{L}_{2}$ analysis in the previous chapter to estimate the new $t-\mathcal{L}_{2}$ gain for the arising hybrid closed loop comprising the $\mathcal{H}_{\infty}$ reset controller and the observer. 
According to 6.1), let us first introduce the plant:

$$
\left[\begin{array}{c|c|c}
A_{p} & B_{p u} & B_{p w} \\
\hline C_{z} & D_{z u} & D_{z w} \\
\hline C_{p} & D_{p u} & D_{p w}
\end{array}\right]=\left[\begin{array}{cc|c|c}
-2.4 & 0 & 2 & 1 \\
1 & 1 & 0 & 1 \\
\hline 0 & 1 & 10 & 0 \\
\hline 0 & 1 & 0 & 5
\end{array}\right] .
$$

The top of Figure 6.3 shows the $t-\mathcal{L}_{2}$ gain obtained for the reset and linear case for a given decay-rate $\alpha$. Similar to Fichera et al. 2012c, the reset controller guarantees lower $t-\mathcal{L}_{2}$ gains than the linear case, as the decay-rate increases. Unlike Fichera et al. 2012c, the design strategy in Section 6.2.2 allows us to design an $\mathcal{H}_{\infty}$ reset controller through a line-search on $\tau_{S} \geq 0$ (see (6.4)). The bottom of Figure 6.3 shows the $t-\mathcal{L}_{2}$ gains obtained with the hybrid synthesis for $\alpha=3$ and for $\tau_{S} \in[0,5]$. Although the $t$ - $\mathcal{L}_{2}$ gain increases with $\tau_{S}$, for $\tau_{S} \geq 1$ we have $\mathcal{H}_{\infty}$ reset controllers with nonstabilizing continuous-time part (see Remark 6.3). Indeed, Figure 6.4(a) shows the behavior of the closedloop system with the $\mathcal{H}_{\infty}$ reset controller obtained with $\alpha=\tilde{\alpha}=3$, $\tau_{S}=5$ and $\rho=5 \cdot 10^{-2}$. Since both the linear and the reset synthesis are designed imposing $\alpha=3$, we do not have any guarantee that the $\mathcal{H}_{\infty}$
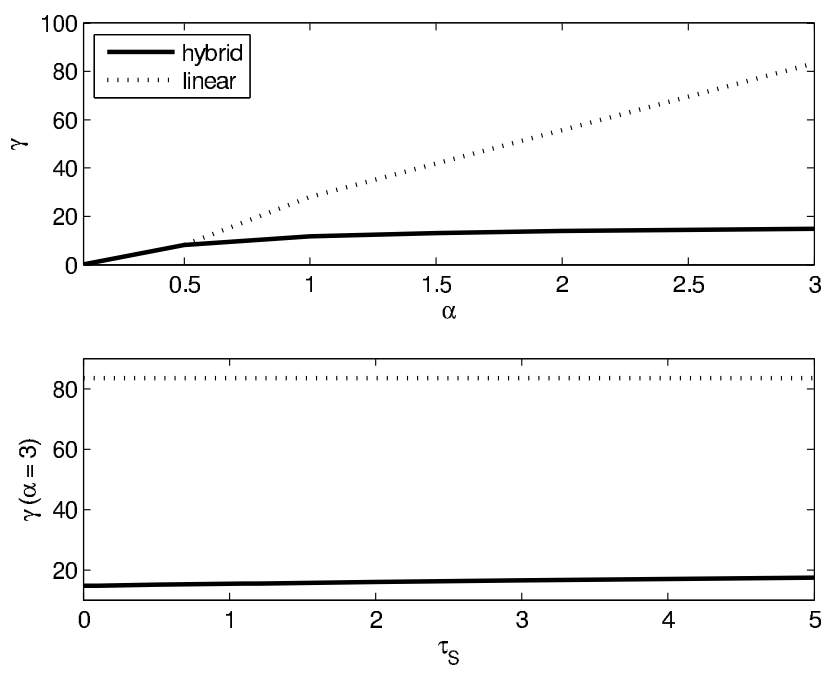

Figure 6.3: Comparison reset $\mathcal{H}_{\infty}$ (solid line) and linear $\mathcal{H}_{\infty}$ (dotted line) control feedback for the DC motor. 

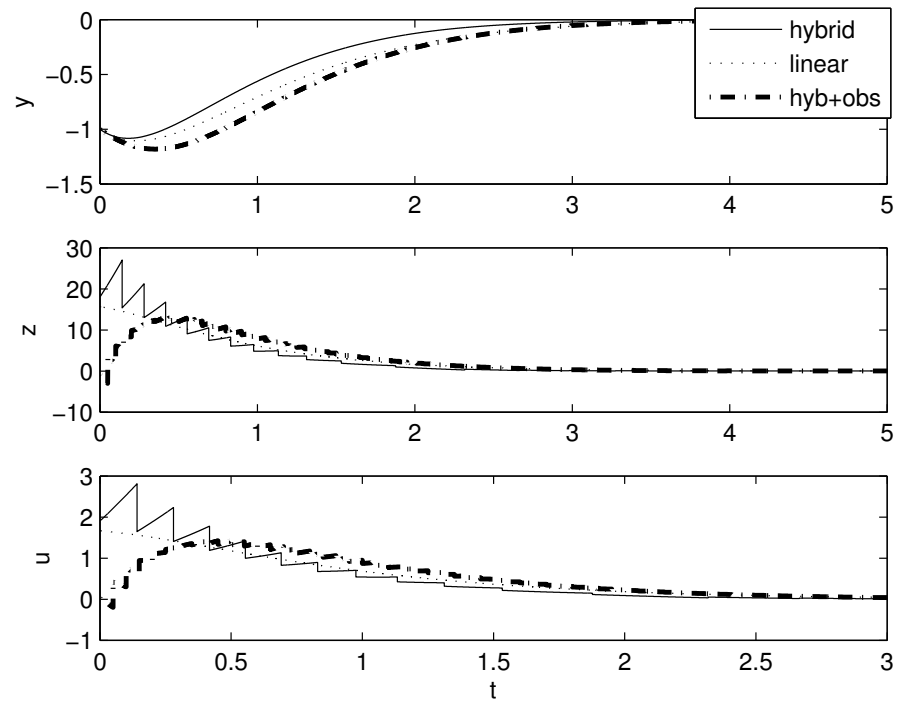

(a) Free response $x_{p}(0,0)=(-1,-1)($ i.e.w $=0)$

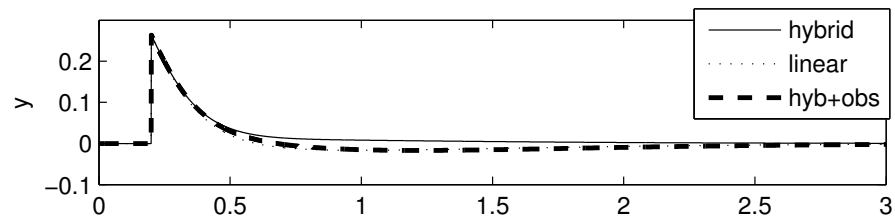

N
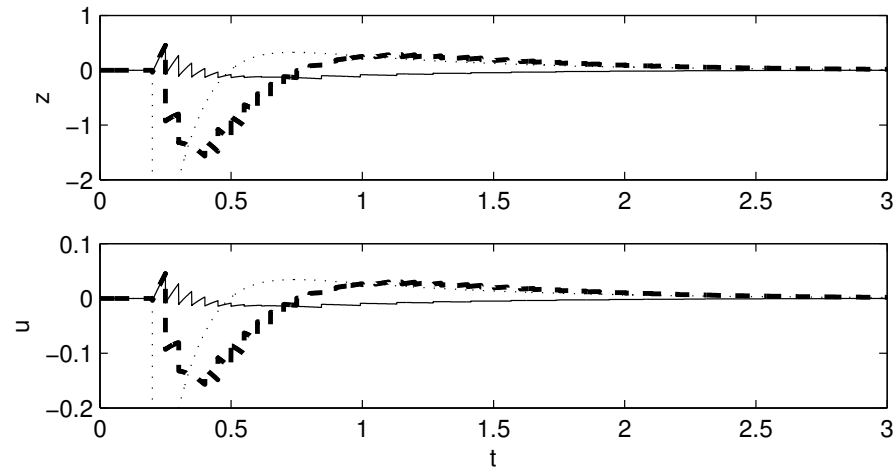

(b) Response with noise $w \in t-\mathcal{L}_{2}$.

Figure 6.4: Simulations for the DC motor, example of Section 6.4 
reset controller leads to faster responses. Nevertheless, the fact that the flow map is unstable requires the action of the reset part to mitigate the unstable modes and to induce exponential stability, with the interesting effects of showing a faster decay rate than the linear case even though the same speed of convergence was imposed by design. In particular according to 6.2 , the synthesis returns the following controller (where $M$ has been divided by its determinant)

$$
\begin{gathered}
{\left[\begin{array}{c|c}
A_{c} & B_{c} \\
\hline K_{p} & - \\
\hline C_{c} & D_{c}
\end{array}\right]=\left[\begin{array}{cc|c}
1.51871 & -1.82471 & 2.17031 \\
0.89613 & 0.67999 & -0.75037 \\
\hline-0.27132 & -0.87136 & - \\
0.60395 & 1.41394 & - \\
\hline 1.85009 & 0.11383 & -0.01509
\end{array}\right],} \\
M=\left[\begin{array}{cccc}
0.00579 & 0.01221 & -0.00579 & -0.01221 \\
0.01221 & 0.02569 & -0.01221 & -0.02569 \\
-0.00579 & -0.01221 & 0.00579 & 0.01221 \\
-0.01221 & -0.02569 & 0.01221 & 0.02569
\end{array}\right] .
\end{gathered}
$$

Figure 6.4 contains also the hybrid output feedback case obtained by applying [Fichera et al. 2013, Theorem 1]. The idea is simply to replace $x_{p}$ by $\hat{x}_{p}$ in (6.2) (flow and jump sets included), where $\hat{x}_{p}$ is the estimated state coming from a classical Luenberger observer Luenberger [1966]:

$$
\dot{\hat{x}}_{p}=\left(A_{p}-L C_{p}\right) \hat{x}_{p}+\left(B_{p u}-L D_{p u}\right) u+L y,
$$

where the observer gain $L=\left[\begin{array}{ll}1.5 & 5.7\end{array}\right]^{\top}$ has been selected by trial and error.

Notice that the multi-objective nature of the synthesis is lost once the observer is introduced because the $t$-decay rate is no longer guaranteed. Nevertheless we can use the analysis developed in the previous chapter to estimate the $t-\mathcal{L}_{2}$ gain of the new hybrid system. By applying Proposition 5.1. we use $(5.13)$ by fixing $a_{3}=1 \cdot 10^{-4}$ and $\rho=1 \cdot 10^{-2}$ and making a line search on $\tau_{S}$. We obtain that the new $t-\overline{\mathcal{L}}_{2}$ gain for the hybrid output feedback is $\gamma=102.86$ (obtained for $\tau_{S}=3$ ). Clearly, an increase of the $t-\mathcal{L}_{2}$ gain is to be expected, as compared to the state feedback case, nevertheless through Proposition 5.1 we are able to still 
establish an upper bound. Figure 6.4 shows a desirable behavior of the $\mathcal{H}_{\infty}$ reset controller, although the case with the observer (bold dashed dot line) is closer to the linear response. The external disturbance $w$ is chosen as $w(t)=\exp (-10 t) \sin (2 t)$, for all $t \geq 0.2$ and zero otherwise.

\subsubsection{Longitudinal dynamics of an F-8 aircraft}
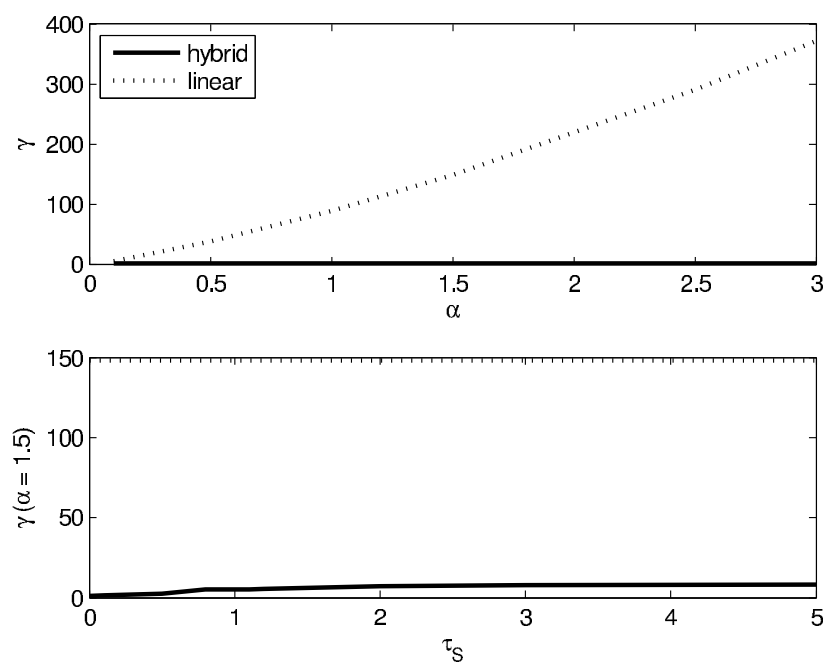

Figure 6.5: Comparison hybrid and linear control feedback for the F-8.

Consider now the following MIMO example, used also in Kapasouris et al. [1988], representing the longitudinal dynamics of the F-8 aircraft.

The system data is

$$
\left[\begin{array}{c}
A_{p} \\
\hline C_{z} \\
C_{p}
\end{array}\right]=\left[\begin{array}{cccc}
-0.8 & -0.0006 & -12 & 0 \\
0 & -0.014 & -16.64 & -32.2 \\
1 & -0.0001 & -1.5 & 0 \\
1 & 0 & 0 & 0 \\
\hline 0 & 0 & 0 & 1 \\
0 & 0 & -1 & 1 \\
\hline 0 & 0 & 0 & 1 \\
0 & 0 & -1 & 1
\end{array}\right],
$$




$$
\left[\begin{array}{c|c}
B_{p u} & B_{p w} \\
\hline D_{z u} & D_{z w} \\
\hline D_{p u} & D_{p w}
\end{array}\right]=\left[\begin{array}{cc|cc}
-19 & -3 & -19 & -3 \\
-0.66 & -0.5 & -0.66 & -0.5 \\
-0.16 & -0.5 & -0.16 & -0.5 \\
0 & 0 & 0 & 0 \\
\hline 1 & 0 & 0 & 0 \\
0 & 1 & 0 & 0 \\
\hline 0 & 0 & 0 & 0 \\
0 & 0 & 0 & 0
\end{array}\right] .
$$

For the purpose of the simulation, we selected a performance output that penalizes both the control input $u$ and the plant output $y$. The four states of this plant represent the pitch rate ( $\mathrm{rad} / \mathrm{sec})$, the forward velocity (ft/sec), the angle of attack ( $\mathrm{rad}$ ) and the pitch angle ( $\mathrm{rad}$ ), respectively. The two measured outputs are then the pitch angle and the flight path angle. The two control inputs (supposed unconstrained) are the aileron angle (deg) and the flaperon angle (deg), respectively.

The top of Figure 6.5 shows the values of $\gamma$ obtained with the linear $\mathcal{H}_{\infty}$ and the reset $\mathcal{H}_{\infty}$ syntheses as a function of the $t$-decay rate, and shows that the $\mathcal{H}_{\infty}$ reset controller induces a certain convergence rate without giving up on the achievable $t$ - $\mathcal{L}_{2}$ gain, which shows a mild increase. The bottom of Figure 6.5 shows that for $\alpha=1.5$, the hybrid synthesis returns $\gamma \simeq 10$ for almost all $\tau_{S} \geq 0$.

Figures 6.6 and 6.7 show the behavior of the $\mathcal{H}_{\infty}$ reset controller obtained for $\alpha=\tilde{\alpha}=1.5$ and $\tau_{S}=5$. The perturbed case is obtained by using the exogenous signal $w=\left[w_{1} w_{2}\right]^{\top}$ defined as

$$
\begin{aligned}
& w_{1}(t)=\left\{\begin{array}{ll}
\exp (-10 t) \sin (2 t) & \text { if } t \geq 2 \\
0 & \text { if } t<2
\end{array},\right. \\
& w_{2}(t)=\left\{\begin{array}{ll}
\exp (-5(t-0.1)) & \text { if } t \geq 0.1 \\
0 & \text { if } t<0.1
\end{array} .\right.
\end{aligned}
$$

We do not report the values of the controller for reason of space. Nevertheless it is easy to see that the controller behaves quite well using much less control than the linear case. In particular, it is possible to see the discontinuous control signal that keeps the trajectories in the flow set guaranteeing a good $t$ - $\mathcal{L}_{2}$ gain and good $t$-decay rate due to the unstable nature of the flow map. 

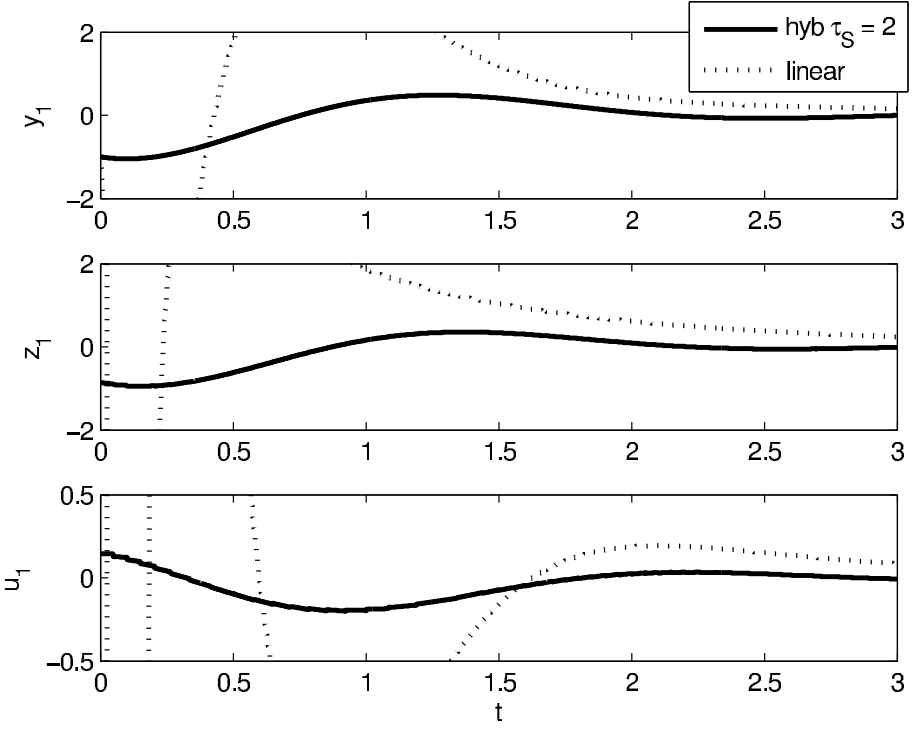

(a) $y_{1}, z_{1}, u_{1}$
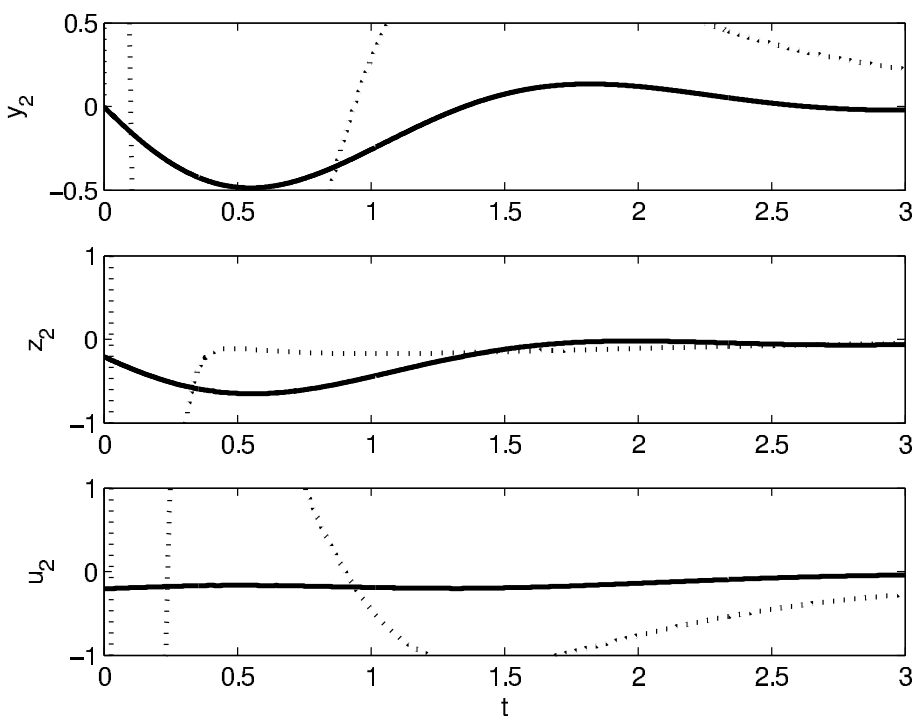

(b) $y_{2}, z_{2}, u_{2}$

Figure 6.6: Free response $x_{p}(0,0)=(-1,-1,-1,-1)($ i.e.w $=0)$. 

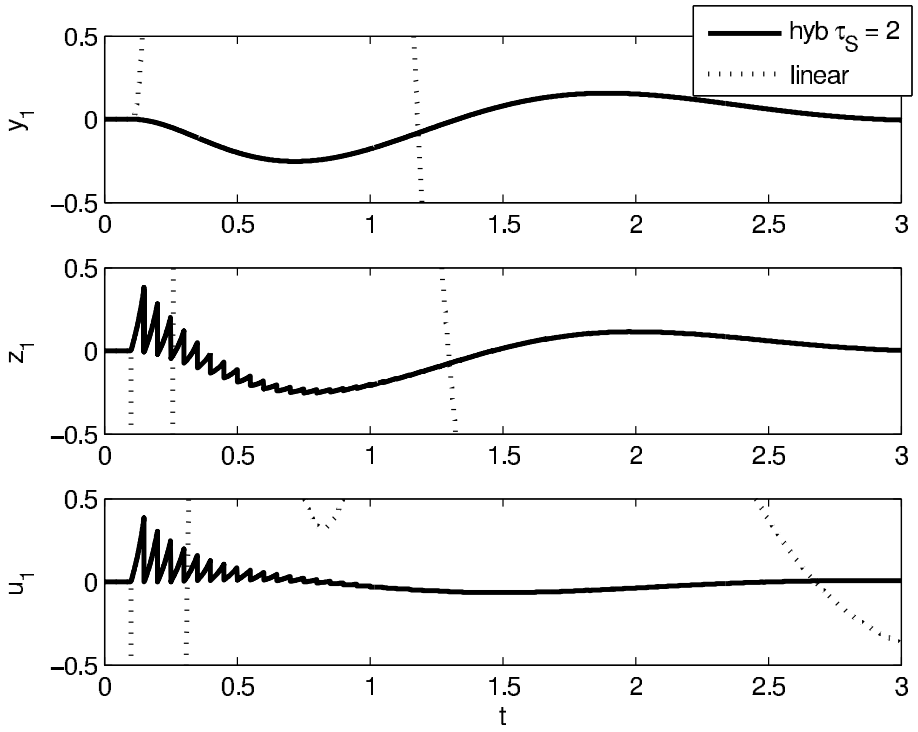

(a) $y_{1}, z_{1}, u_{1}$
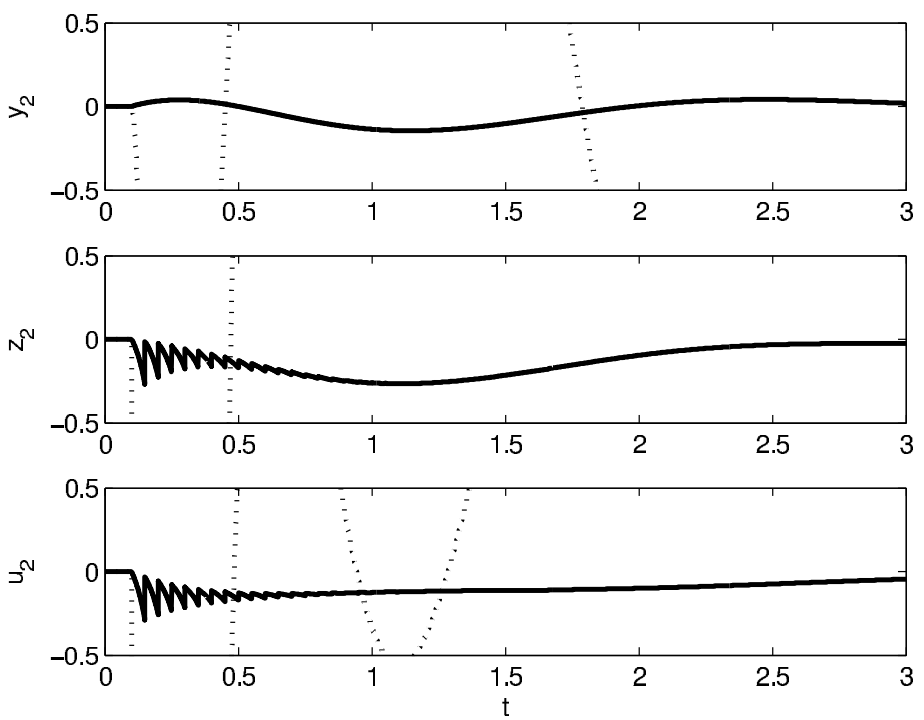

(b) $y_{2}, z_{2}, u_{2}$

Figure 6.7: Response with noise $w \in t-\mathcal{L}_{2}$. 


\section{Part III}

\section{Planar reset systems}




\section{Planar SISO systems with First Order Reset Elements}

\subsection{A modified model of FORE and its Lyapunov implications}

In this chapter we come back to the material initially introduced in Part I of the survey (namely Chapters 1 and 2 ) and address, first, the issue of stability assessment and regulation for the simple case where a First Order Reset Element (FORE) controls a first order plant. The arising closed loop is planar and this fact simplifies the stability conditions to the point that necessary and sufficient conditions can be established.

The results presented in this chapter actually pertain a revised model of FORE, that was introduced in Nešić et al. [2011], which corresponds to replacing the jump and flow conditions in (1.2) as follows:

$$
\begin{cases}\dot{x}_{c}=a_{c} x_{c}+b_{c} e, & \text { is allowed when } \varepsilon v^{2}+2 e x_{c} \geq 0 \\ x_{c}^{+}=0, & \text { is allowed when } \varepsilon v^{2}+2 e x_{c} \leq 0,\end{cases}
$$

where $\varepsilon>0$ is a small number associated with the tilting of the flow set boundary (see Figures 7.1 and 7.2. . One way to understand the different FORE models $(1.2)$ and 7.1 is by comparing the different sets $\mathcal{F}$ and $\mathcal{J}$ enabling the possibility of flowing or jumping for the corresponding solutions.

Then, at any time $(t, j) \in \operatorname{dom}\left(x_{c}\right)$, a solution of the hybrid system 


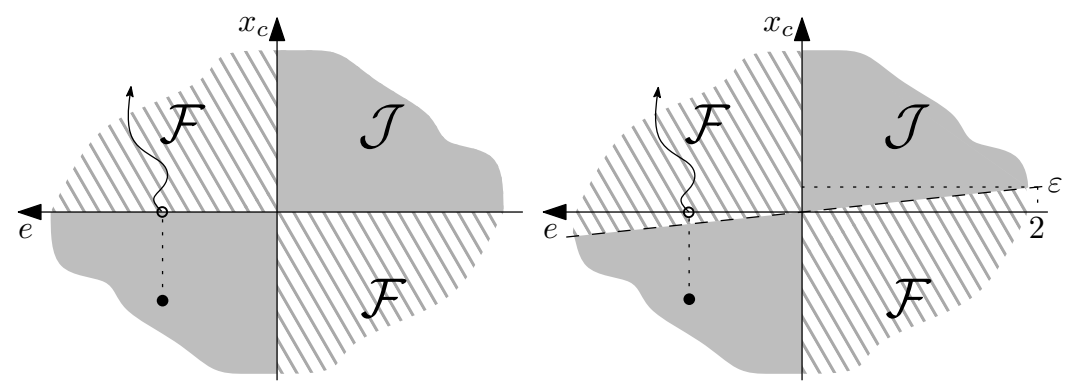

Figure 7.1: The jump (grey) and flow (striped) sets for the model 1.2 (left) and the modified model (7.1) (right).

will flow or jump depending on whether its value at that time belongs to the jump set $\mathcal{J}$ or it belongs to the flow set $\mathcal{F}$ or even both.

With reference to Figure 1.4. Figure 7.1 shows the differences between the flow sets $\mathcal{F}$ and jump sets $\mathcal{J}$ for the two FORE models 1 (1.2) and (7.1). For instance, in the FORE model (1.2) the jump set $\mathcal{J}$ consists of the first and third quadrants including the axes and the flow set is the second and fourth quadrant including the axes (left subfigure). Note that in this case $\mathcal{J} \cap \mathcal{F}=\left\{\left(x_{c}, e\right): x_{c}=0\right.$ or $\left.e=0\right\}$. The right sub-figure corresponds to the modified FORE model and the only difference is that one of the boundaries of the jump and flow sets is slightly tilted. A sample trajectory starting from the same initial condition and with the same input is given in all two sub-figures (see also the one already discussed in Figure 1.4 to illustrate the difference in dynamic behavior that comes from the differences among the jump and flow sets. In particular, note that the modified model has the advantage that solutions jumping from the interior of $\mathcal{J}$ are mapped to the interior of $\mathcal{F}$. This fact leads to several advantages when wanting to prove suitable Lyapunov conditions for closed loops involving FOREs.

Paralleling the discussions in Chapter 4 and in the following chapters, we note that Zeno solutions exist also for the modified model, oc-

\footnotetext{
${ }^{1}$ In Figure 7.1, the $e$ axis direction is reversed so that in the case of negative feedback with one dimensional plants $\left(v=-x_{p}\right)$, this is exactly the closed-loop phase plane, which has been commented in Nešić et al. 2008b, 2005, Zaccarian et al. 2005, 2006, Nešić et al. 2008a], Zaccarian et al. [2011].
} 
curring whenever the state jumps from the origin of the $\left(x_{c}, e\right)$ plane. Then, in order to avoid Zeno solutions, we augment (7.1) with a clock variable in a similar manner to (4.1), resulting in:

$$
\begin{aligned}
& \left\{\begin{array}{l}
\dot{\tau}=1-\mathrm{dz}\left(\frac{\tau}{\rho}\right), \\
\dot{x}_{c}=a_{c} x_{c}+b_{c} e,
\end{array}\right. \\
& \left\{\begin{array}{l}
\tau^{+}=0, \\
x_{c}^{+}=0,
\end{array}\left(e, x_{c}\right) \in \mathcal{F} \text { or } \tau \in[0, \rho],\right.
\end{aligned}
$$

with

$$
\begin{aligned}
& \mathcal{F}:=\left\{\left(e, x_{c}\right): \varepsilon e^{2}+2 e x_{c} \geq 0\right\}, \\
& \mathcal{J}:=\left\{\left(e, x_{c}\right): \varepsilon e^{2}+2 e x_{c} \leq 0\right\},
\end{aligned}
$$

The motivation for using (7.1) as opposed to 1.2 stems from a Lyapunov analysis that is discussed next. When using the temporally regularized model (4.1) the temporal regularization causes the solutions to possibly slightly overflow into the jump set $\mathcal{J}$ before the reset occurs and this makes the Lyapunov construction harder. In particular, all the first Lyapunov-based results reported in Nešić et al. 2008a, 2005, 2008b, Zaccarian et al. 2005, 2006, 2007, 2011 (which are established for model (4.1) and well summarized in Chapter 5) are based on the existence of a Lyapunov function that satisfies standard regularity and growth conditions, in addition to the following flow and jump conditions:

1. it is a disturbance attenuation Lyapunov function in a slightly inflated version of the flow set $\mathcal{F}$;

2. it does not increase when jumping from the jump set $\mathcal{J}$.

The two requirements above are graphically represented in the left sub-figure of Figure 7.2. The striped region represents the set on which the Lyapunov function is required satisfy item 1 (the flow conditions) and the shaded region is where item 2 above (jump conditions) should hold. Note that there is an overlap of these two sets where both items 1 and 2 should hold. This stringent requirement makes the construction of appropriate Lyapunov functions hard in this case. 

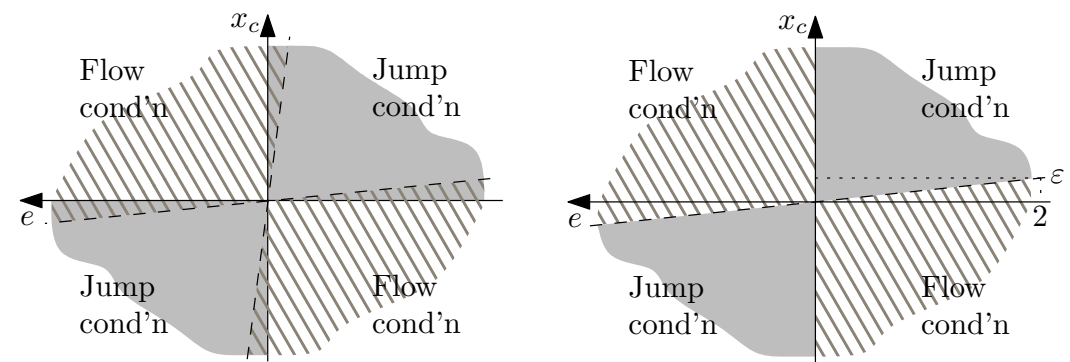

Figure 7.2: The sectors of the state space where the Lyapunov flow and jump conditions must hold for the temporally regularized FORE 1.2 without strict decrease at jumps (left) and the FORE $\sqrt{7.2}$ with strict decrease at jumps (right).

Conversely, the stability results for reset systems that use the model (7.1) and its temporally regularized version 7.2 require a Lyapunov function that:

1a. is a disturbance attenuation Lyapunov function in the flow set $\mathcal{F}$;

2a. strictly decreases when jumping from the jump set $\mathcal{J}$.

The right sub-figure of Figure 7.2 illustrates the two new conditions. The striped and shaded regions represent the sets on which the Lyapunov function needs to satisfy items $1 \mathrm{a}$ and $2 \mathrm{a}$ respectively. It turns out that it is much easier to construct Lyapunov functions satisfying conditions $1 \mathrm{a}$ and $2 \mathrm{a}$ rather than 1 and 2 . Indeed, note that the model (1.2) as it stands will never satisfy item 2a because there are cases when a state jumps onto itself (this is whenever $x_{c}=0$ ). Therefore it is necessary to tilt the boundary between the flow and the jump set as shown in the right sub-figure of Figure 7.1. More specifically, the "tilting" corresponds to transforming the horizontal sector boundary from $x_{c}=0$ (i.e., the $e$ axis) into $x_{c}=-\varepsilon e$. Then any state in the jump set will be mapped into the interior of the flow set (except for the origin) and it will be possible to construct Lyapunov functions guaranteeing item $2 \mathrm{a}$.

From an intuitive viewpoint, having a strict decrease at jumps allows to compensate for a possible growth of $V(x(t))$ that might have happened while $x(t)$ was overflowing in the jump set (for $\tau \leq \rho$ ), thus 
making it possible to require a less stringent flow condition and to only rely on the regularity assumptions on $V$.

\subsection{Necessary and sufficient conditions for exponential sta- bility}

In this section we characterize the exponential and $t$ - $\mathcal{L}_{2}$ stability properties of the planar reset systems that consist of the FORE (7.2), interconnected via

$$
u=x_{c}, \quad e=-y
$$

to the following scalar linear plant

$$
\left\{\begin{aligned}
\dot{x}_{p} & =a_{p} x_{p}+b_{p} u+d \\
y & =x_{p}
\end{aligned}\right.
$$

where $u \in \mathbb{R}$ is the control input, $d \in \mathbb{R}$ is a disturbance input and $x_{p} \in \mathbb{R}$ is the plant state.

In particular, in Theorem 7.1 we characterize asymptotic and exponential stability of the closed-loop giving necessary and sufficient conditions in terms of the system parameters. Then in Theorems 7.2 and 7.3 we provide results about the $t-\mathcal{L}_{2}$ gain of the planar reset system from the input $d$ to the output $y$.

The closed loop (7.4), (7.2), (7.3) can be conveniently written in the following form

$$
\begin{aligned}
& \begin{cases}\dot{\tau}=1-\mathrm{dz}\left(\frac{\tau}{\rho}\right), & x^{\top} M x \leq 0 \text { or } \tau \in[0, \rho], \\
\dot{x}=A x+B d, & \end{cases} \\
& \left\{\begin{array}{l}
\tau^{+}=0, \\
x^{+}=G x,
\end{array} \quad x^{\top} M x \geq 0 \text { and } \tau \in[\rho, 2 \rho],\right.
\end{aligned}
$$

where $x:=\left(x_{p}, x_{c}\right) \in \mathbb{R}^{2}$ and

$$
\left[\begin{array}{c|c}
A & B \\
\hline G & M
\end{array}\right]=\left[\begin{array}{cc|cc}
a_{p} & b_{p} & 1 & \\
-b_{c} & a_{c} & 0 & \\
\hline 1 & 0 & -\varepsilon & 1 \\
0 & 0 & 1 & 0
\end{array}\right] .
$$


where the sign in the selection of $M$ comes from the negative feedback interconnection in (7.3) (see also the right sub-figure of Figure 7.1). To state a stability result concerning the FORE control system 7.5 , (7.6) we introduce the following definition.

Definition 7.1. Consider the FORE control system with temporal regularization (7.5), (7.6). The system is exponentially stable (or finite gain $t-\mathcal{L}_{2}$ stable) conditionally to hierarchically small $(\varepsilon, \rho)$ if there exists $\varepsilon^{*}$ such that for each $\varepsilon \in\left(0, \varepsilon^{*}\right]$ there exists $\rho^{*}$ such that for all $\rho \in\left(0, \rho^{*}\right]$ we have that system $(7.5),(7.6)$ is exponentially stable (finite gain $t-\mathcal{L}_{2}$ stable).

The next theorem establishes necessary and sufficient conditions for the exponential stability and finite $t$ - $\mathcal{L}_{2}$ gain (from $d$ to $x$ ) of the planar FORE control system (7.5), (7.6). The result was presented in Nešić et al. 2011.

Theorem 7.1. (Stability conditions) Consider the planar FORE control system (7.5), (7.6) (i.e., the closed-loop system (7.4), (7.2), (7.3p) and suppose that the loop gain $b_{p} b_{c}$ is positive. Then the following statements are equivalent.

1. The origin is (locally) asymptotically stable conditionally to hierarchically small $(\varepsilon, \rho)$.

2. The origin is globally exponentially stable conditionally to hierarchically small $(\varepsilon, \rho)$.

3. The system is finite gain $t-\mathcal{L}_{2}$ and $t-\mathcal{L}_{2}$ to $t-\mathcal{L}_{\infty}$ stable from $d$ to $\left(x_{p}, x_{c}\right)$ conditionally to hierarchically small $(\varepsilon, \rho)$.

4. At least one of the following two conditions holds:

(a) the matrix $A$ in 7.6 is Hurwitz;

(b) the following condition is satisfied:

$$
2 \sqrt{b_{p} b_{c}}+a_{c}-a_{p}>0
$$


Theorem 7.1 establishes necessary and sufficient conditions for exponential stability and $t-\mathcal{L}_{2}$ stability of planar FORE systems. In particular, from item 4a it follows that for a planar FORE control system, as long as the loop gain is positive (thereby leading to a negative feedback because $v=-y$ ), FORE resets can never destabilize an underlying exponentially stable linear dynamics. An interesting aspect to study is to understand how the $t$ - $\mathcal{L}_{2}$ gain compares to the $t$ - $\mathcal{L}_{2}$ gain of the closed-loop without resets (whenever it exists) and also the trend of the gain as certain parameters get large. In particular, it was commonly acknowledged by practical experience of the early 2000 that introducing resets improves the performance of a linear planar control system, even though a formal proof of this fact was not available. A possible motivation for this fact can be found in the next Theorem 7.2, which was presented in Nešić et al. 2011.

Moreover, it has been already noticed by studying certain gain estimates in the numerical examples reported in Chapter 5 that the $t$ - $\mathcal{L}_{2}$ gain seems to become smaller as the loop gain and/or the pole of the FORE (namely $a_{c}$ ) becomes larger. This intuition arises from the fact that the step response generated by the closed loop is faster, by corresponding to the patching of an exponentially diverging branch (having larger growth rate) followed by a flat-top at the desired steady state (see also the blue response in Figure 2.2 or the response of Figure 4.4 when using an observer). A formal proof of these $t$ - $\mathcal{L}_{2}$ gain trends has been given in Nešić et al. 2011] through the theorems reported below. For the correct statement of these theorems we need to clarify a suitable concept of gain estimate and of gain convergence, introduced in the next definition.

Definition 7.2. Consider the FORE control system with temporal regularization (7.5), (7.6). Assume that $\gamma$ is an input/output gain. Then we say that $\bar{\gamma}$ is an asymptotic estimate of the gain $\gamma$ conditionally to hierarchically small $(\varepsilon, \rho)$, denoted as follows:

$$
\gamma \stackrel{\varepsilon, \rho}{\leq} \bar{\gamma}
$$

if for each (arbitrarily small) $\delta>0$ there exists $\varepsilon^{*}$ such that for each $\varepsilon \in\left(0, \varepsilon^{*}\right]$ there exists $\rho^{*}$ such that for all $\rho \in\left(0, \rho^{*}\right], \gamma \leq \bar{\gamma}+\delta$. 
Assume that $p$ is a suitable parameter of the closed-loop system and that $\gamma(p)$ is an input/output gain depending on $p$. Then we say that $\gamma(p)$ converges to zero conditionally to hierarchically small $(\varepsilon, \rho)$ as $p$ tends to $+\infty$, denoted as follows:

$$
p \rightarrow \infty \Rightarrow \gamma(p) \stackrel{\varepsilon, \rho}{\longrightarrow} 0,
$$

if for each (arbitrarily small) $\bar{\gamma}>0$ there exists ${ }^{2} p^{*}>0$ such that for each $p \geq p^{*}$ there exists $\varepsilon^{*}$ such that for each $\varepsilon \in\left(0, \varepsilon^{*}\right]$ there exists $\rho^{*}$ such that for all $\rho \in\left(0, \rho^{*}\right], \gamma(p) \leq \bar{\gamma}$.

Remark 7.1. The goal of Definition 7.2 is to clarify what we mean by gain estimate and convergence to a value in terms of the small parameters of the system. In particular, the gain estimates and trends established in the next theorem require that first the parameter $\varepsilon$ characterizing the FORE resetting rule in $(7.2)$ is sufficiently small and then that the temporal regularization constant $\rho$ is once again sufficiently small. This hierarchical selection is necessary because larger (possibly unstable) FORE poles will cause larger state evolution and smaller selections of $\rho$ will be necessary. However, for fixed parameters, there always exists a small enough $\rho$ for which the theorem statements hold. Similarly, with reference to the second part of Definition 7.2 , we note that in Theorem 7.3 we consider various situations when $p=a_{c}$ or $p=k:=b_{c} b_{p}$ or $p=\left(a_{c}, k\right)$. In a design context, one should first fix the desired gain $\bar{\gamma}$, then choose $p$ sufficiently large and then impose first $\varepsilon$ sufficiently small and subsequently $\rho$ sufficiently small.

Theorem 7.2. $\left(t-\mathcal{L}_{2}\right.$ gain estimates $)$ Consider the planar FORE control system (7.5), (7.6) (i.e., the closed-loop system (7.4), 7.2), 7.3) with temporal regularization) where the loop gain $k:=b_{p} b_{c}$ is positive. Whenever the closed loop is exponentially stable (so that, by Theorem 7.1 at least one of the two conditions at item 4 of Theorem 7.1 holds), the following asymptotic estimates hold conditionally to hierarchically small $(\varepsilon, \rho)$ (in the sense of Definition 7.2 for the $t-\mathcal{L}_{2}$ gain $\gamma$ of the closed loop from $d$ to $y$ :

\footnotetext{
${ }^{2}$ The parameter $p$ is allowed to be a vector and in this case $p>0$ means that each entry of $p$ is strictly larger than zero.
} 
1. if item 4a of Theorem 7.1 holds, then

$$
\gamma \stackrel{\varepsilon, \rho}{\leq} \gamma_{L}
$$

where $\gamma_{L}$ is the (finite, because $A$ is Hurwitz) gain from $d$ to $y$ of the linear closed-loop without resets.

2. if item $4 \mathrm{~b}$ of Theorem 7.1 holds, then

$$
\gamma \stackrel{\varepsilon, \rho}{\leq} \frac{2(2+\kappa) \exp \left(\kappa \frac{\pi}{2}\right)}{\kappa\left(2 \sqrt{b_{c} b_{p}}-\max \left\{a_{p}-a_{c}, 0\right\}\right)-4 \max \left\{\left|a_{c}\right|,\left|a_{p}\right|\right\}},
$$

where $\kappa$ is any constant satisfying $\kappa>\bar{\kappa}:=\frac{4 \max \left\{\left|a_{c}\right|,\left|a_{p}\right|\right\}}{2 \sqrt{b_{c} b_{p}}-\max \left\{a_{p}-a_{c}, 0\right\}}$.

Remark 7.2. It is of interest to investigate whether for fixed values of the parameters there is an optimal selection of $\kappa$ within $(7.9)$ which gives the tightest estimate for the $t$ - $\mathcal{L}_{2}$ gain. Indeed, by taking the derivative of the right-hand side of $(7.9)$ with respect to $\kappa$ and imposing that the derivative is zero, one gets two solutions (of a second order equation), one of them always being smaller than $\bar{\kappa}$ (thus not being usable) and one of them always being larger than $\bar{\kappa}$. In particular, the optimal $\kappa$ is determined as $\kappa^{*}:=\frac{\bar{\kappa}}{2}-1+\sqrt{\left(\frac{\bar{\kappa}}{2}+1\right)\left(\frac{\bar{\kappa}}{2}+1+\frac{4}{\pi}\right)}$, which is a global minimizer because (7.9) is a smooth function of $\kappa$ growing unbounded both when $\kappa \rightarrow \bar{\kappa}$ from the right and when $\kappa \rightarrow+\infty$. When substituted into the gain bound equation $(7.9)$, this value $\kappa^{*}$ gives the following bound, which only depends on the system parameters:

$$
\gamma^{*}=\frac{1+\kappa_{0}+\sqrt{\kappa_{0}\left(\kappa_{0}+2\right)} \exp \left(\kappa_{1}+\sqrt{\kappa_{0}\left(\kappa_{0}+2\right)}\right)}{2 \sqrt{b_{c} b_{p}}-\max \left\{a_{p}-a_{c}, 0\right\}}
$$

where $\kappa_{0}=\frac{\pi}{4}(\bar{\kappa}+2)$ and $\kappa_{1}=\frac{\pi}{4}(\bar{\kappa}-2)$.

An example of the gain curve given by the function 7.10 is shown in Figure 7.3. when selecting $a_{p}=0$ and $b_{p} b_{c}=1$ and having $a_{c}$ take values in $[-0.4,0.4]$. This curve is compared to the gain estimates obtained when using the analytic and numerical tools given in Theorem 5.4 and Theorem 5.5, respectively (this comparison had appeared in Zaccarian et al. 2011]). The latter estimates turn out to be tighter for this special case, but the advantage of this construction is that it provides an 


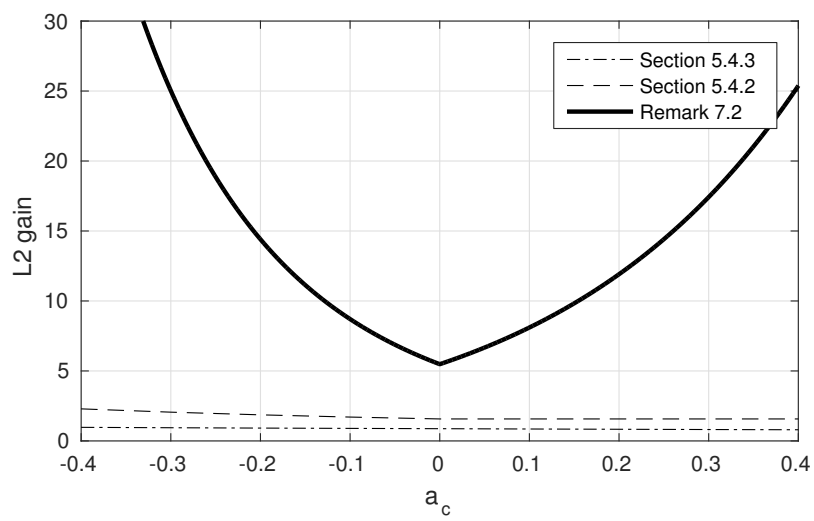

Figure 7.3: Comparison of the gain estimates obtained by using equation 7.10 from Remark 7.2 (bold), using the analytic tools from Section 5.4 .2 (dashed) and the numerical tools from Section 5.4.3 (dash-dotted).

estimate of the gain for a larger class of systems (the construction in Theorem 5.4 is limited to the case $a_{p}=0$ and $b_{p} b_{c}=1$ ).

Theorem 7.3. $\left(t-\mathcal{L}_{2}\right.$ gain trends) Consider the planar FORE control system (7.5), (7.6) (i.e., the closed-loop system (7.4), (7.2), (7.3)) where the loop gain $k:=b_{p} b_{c}$ is positive. Let $a_{p}$ be fixed. Denote by $\gamma\left(a_{c}, k\right)$ the $t-\mathcal{L}_{2}$ gain of the closed-loop from $d$ to $y$ as a function of the FORE pole $a_{c}$ and of the loop gain $k:=b_{p} b_{c}$. Then the following trends hierarchically conditioned by $(\varepsilon, \rho)$ in the sense of Definition 7.2 hold for the closed-loop system:

1. $k \rightarrow+\infty \Rightarrow \gamma\left(a_{c}, k\right) \stackrel{\varepsilon, \rho}{\longrightarrow} 0$,

2. $a_{c} \rightarrow+\infty \Rightarrow \gamma\left(a_{c}, k\right) \stackrel{\varepsilon, \rho}{\longrightarrow} 0$,

3. $k \rightarrow+\infty$ and $a_{c} \rightarrow+\infty \Rightarrow \gamma\left(a_{c}, k\right) \stackrel{\varepsilon, \rho}{\longrightarrow} 0$,

namely, the $t-\mathcal{L}_{2}$ gain of the closed loop decreases to zero (conditionally to hierarchical selections of $(\varepsilon, \rho))$ as the loop gain and/or the FORE pole are increased. 


\subsection{Extension to minimum phase relative degree one linear SISO plants}

The stability results of the previous section can be exploited for showing stability of a larger class of SISO linear systems that can be represented as the feedback interconnection between a planar FORE closed loop and an auxiliary exponentially stable linear dynamics. The approach is similar in spirit to the well-known high gain stabilization results for linear systems, but it is enhanced by the extra property that not only high gain stabilizes the planar FORE closed loop but, as an alternative, highly unstable FOREs stabilize the closed loop as well.

The underlying idea in the $t$ - $\mathcal{L}_{2}$ stability proof is to view the control system as a feedback interconnection of a planar reset control system and an asymptotically stable linear subsystem as depicted in Figure 7.4 and then use a small gain theorem, exploiting the $t$ - $\mathcal{L}_{2}$ gain results for planar reset systems reported in Section 7.2. Then using the fundamental stability results already discussed in Section 4.4 , which pertain to general linear reset systems, we get exponential stability in the absence of disturbances.
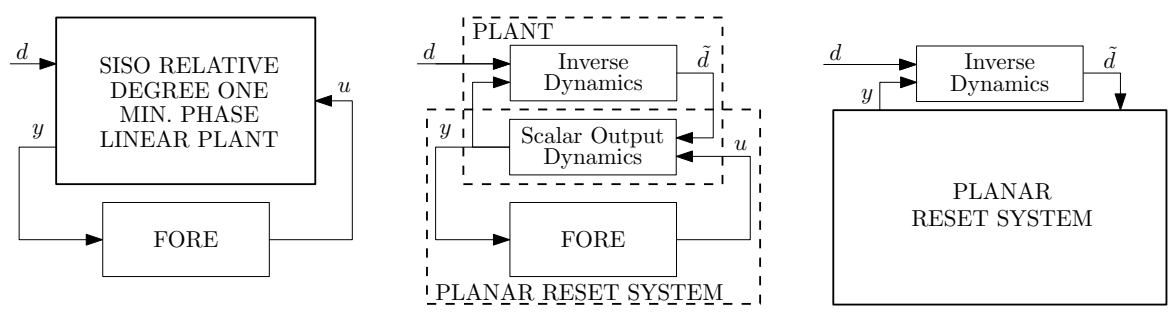

Figure 7.4: Equivalent feedback interconnections for stability analysis purposes.

More specifically, let us consider a higher order linear plant generalizing the scalar dynamics (7.4) as follows:

$$
\mathcal{P}\left\{\begin{aligned}
\dot{x}_{p} & =A_{p} x_{p}+B_{p u} u+B_{p d} d \\
y & =C_{p} x_{p}
\end{aligned}\right.
$$

where $x_{p} \in \mathbb{R}^{n_{p}}$ is the plant state, $u \in \mathbb{R}$ is the control input, $d \in \mathbb{R}^{n_{d}}$ is a disturbance input and $y \in \mathbb{R}$ is the measured plant output $\left(A_{p}\right.$, $B_{p u}, B_{p d}$ and $C_{p}$ are matrices of appropriate dimensions). 


\subsection{Extension to minimum phase relative degree one linear SISO plab4I}

Under a minimum phase and relative degree one assumption, there exists a nonsingular change of coordinates so that we can write dynamics 7.11 as Isidori, 1995, Remark 4.3.1]:

$$
\begin{aligned}
& \dot{z}=A_{z} z+B_{z y} y+B_{z d} d \\
& \dot{y}=a_{p} y+b_{p} u+\underbrace{C_{z} z+E_{d} d}_{\tilde{d}},
\end{aligned}
$$

where $y \in \mathbb{R}$ corresponds to the scalar output dynamics, $z \in \mathbb{R}^{n_{p}-1}$ corresponds to the inverse dynamics and $u \in \mathbb{R}$ is the plant input in the middle diagram of Figure 7.4. Since plant (7.11) is minimum phase, $A_{z}$ is Hurwitz and we assume without loss of generality that $b_{p}>0$. We may now generalize the hierarchical concepts introduced in Definition 7.1 for our characterization of stability, with the extra feature of studying trends of certain closed-loop parameters (such as the loop gain or the pole of the FORE).

Definition 7.3. Consider the FORE control system with temporal regularization (7.11), (7.2), (7.3). Assume that $\alpha$ is a suitable parameter of the closed-loop system. Then we say that the system is exponentially stable (or finite gain $t-\mathcal{L}_{2}$ stable) conditionally to large $\alpha$ and hierarchically small $(\varepsilon, \rho)$ if there exists $\alpha^{*}>0$ such that for each $\alpha \geq \alpha^{*}$ there exists $\varepsilon^{*}$ such that for each $\varepsilon \in\left(0, \varepsilon^{*}\right]$ there exists $\rho^{*}$ such that for all $\rho \in\left(0, \rho^{*}\right]$ we have that system $(7.11),(7.2),(7.3)$ is exponentially stable (finite gain $t$ - $\mathcal{L}_{2}$ stable).

It is understood in the above definition that the only parameters that we can change are $\alpha, \varepsilon, \rho$, whereas all other constants in the model are fixed. Then we can state the following result, which has been proven in Nešić et al. 2011].

Theorem 7.4. Consider the closed loop between the plant $\sqrt{7.12})$ and the FORE (7.2) via the interconnection $(7.3)$, where the FORE (7.2) is parametrized by $\left(a_{c}, b_{c}, \varepsilon, \rho\right)$. Let $A_{z}$ be Hurwitz and $b_{p}>0$ in (7.12). Then, the following statements are true:

1. (unstable FORE feedback) the system is finite gain $t-\mathcal{L}_{2}$ and $t-\mathcal{L}_{2}$ to $t$ - $\mathcal{L}_{\infty}$ stable from $d$ to $x_{p}$ conditionally to large $a_{c}$ and hierarchically small $(\varepsilon, \rho)$. Moreover, when $d(t) \equiv 0$ the system is also 
exponentially stable conditionally to large $a_{c}$ and hierarchically small $(\varepsilon, \rho)$;

2. (high gain feedback) the system is finite gain $t$ - $\mathcal{L}_{2}$ and $t-\mathcal{L}_{2}$ to $t-\mathcal{L}_{\infty}$ stable from $d$ to $x_{p}$ conditionally to large $b_{c}$ and hierarchically small $(\varepsilon, \rho)$. Moreover, when $d(t) \equiv 0$ the system is also exponentially stable conditionally to large $b_{c}$ and hierarchically small $(\varepsilon, \rho)$;

3. (high gain+unstable FORE feedback) the system is finite gain $t-\mathcal{L}_{2}$ and $t$ - $\mathcal{L}_{2}$ to $t-\mathcal{L}_{\infty}$ stable from $d$ to $x_{p}$ conditionally to large $\left(a_{c}, b_{c}\right)$ and hierarchically small $(\varepsilon, \rho)$. Moreover, when $d(t) \equiv$ 0 the system is also exponentially stable conditionally to large $\left(a_{c}, b_{c}\right)$ and hierarchically small $(\varepsilon, \rho)$.

Remark 7.3. The three results in Theorem 7.4 can be interpreted in the context of the well-known high-gain feedback stabilization of linear systems. In particular, item 2 states that the same result holds for reset control systems. The novelty established here is in item 1 which states that high instability in the FORE is capable of stabilizing the reset control system. This result is new and significantly less trivial because the underlying linear dynamics become exponentially unstable for large values of $a_{c}$. Despite this fact, our novel proof technique allows to establish the exponential stability of the reset control system. Finally, item 3 simply states that if both the loop gain and the FORE pole go to infinity, the same stabilization result still holds.

Remark 7.4. A result similar to Theorem 7.4 can be proved under appropriate conditions for a class of nonlinear SISO systems that are minimum phase (in an appropriate sense) and relative degree one. For instance, consider a nonlinear control affine system without disturbances:

$$
\left\{\begin{array}{l}
\dot{x}_{p}=f\left(x_{p}\right)+g\left(x_{p}\right) u_{p} \\
y=h\left(x_{p}\right)
\end{array}\right.
$$

and suppose that there exists a (global or local) nonsingular change of coordinates $(z, y)=T\left(x_{p}\right)$ and an input transformation $u_{p}=K\left(x_{p}\right)+$ $L\left(x_{p}\right) u$ such that the system in new coordinates and with the new input 


\subsection{Extension to minimum phase relative degree one linear SISO plab43}

$u$ becomes 3

$$
\left\{\begin{array}{l}
\dot{z}=F(z, y) \\
\dot{y}=u+G(z, y) .
\end{array}\right.
$$

In other words, the nonlinear system is input-output linearized. Then, if we assume that the zero dynamics, which correspond to the $z$ state are finite gain $\mathcal{L}_{2}$ stable from $y$ to $G(z, y)$, we can apply FORE design to the linearized $y$ state and use the same steps to conclude that increasing the gain or the pole (or both) of the FORE would stabilize the overall nonlinear system.

Remark 7.5. Our results can be generalized to a class of nonlinear MIMO plants that have the same number $\chi$ of inputs and outputs if there exists a coordinate and input transformation that yields the system in the following form:

$$
\left\{\begin{aligned}
\dot{z} & =F(z, y) \\
\dot{y}_{i} & =u_{i}+G_{i}(z, y), i=1, \ldots, \chi
\end{aligned}\right.
$$

For precise conditions under which such transformations are possible for control affine systems, see [Isidori, 1995, Chapter 5]; typically, one would require vector relative degree $(1,1, \ldots, 1)$ and zero dynamics that are stable in an appropriate sense. In this case, applying a FORE to each SISO pair $\left(y_{i}, u_{i}\right)$ we will obtain the planar system considered in the next section and the overall system consists of $\chi$ such decentralized systems interconnected in feedback with the zero dynamics. With appropriate stability properties on the zero dynamics and by adjusting the parameters of all FOREs we can show that stability of the closed loop holds.

While the results commented above are useful for stability assessment when using FORE stabilizers, one important aspect that should be recognized is that unlike PI-type control laws, FOREs are not capable of rejecting constant disturbances such as sensor biases and/or actuator offsets, which are pervading in control systems technology. Due to this fact, the discussion of the next sections is geared towards

\footnotetext{
${ }^{3}$ Conditions under which this transformation is possible are given for instance in Isidori, 1995, Chapter 4].
} 
first establishing some results about constant set-point regulation, and then also non-constant reference tracking.

\subsection{Set-point regulation}

\subsubsection{Constant feedforward injection}

The goal of this section is to show how the set-point regulation of plant (7.11) using a FORE can be reduced to a stabilization problem in suitably transformed coordinates. A block diagram of the arising control scheme is represented in Figure 7.5.

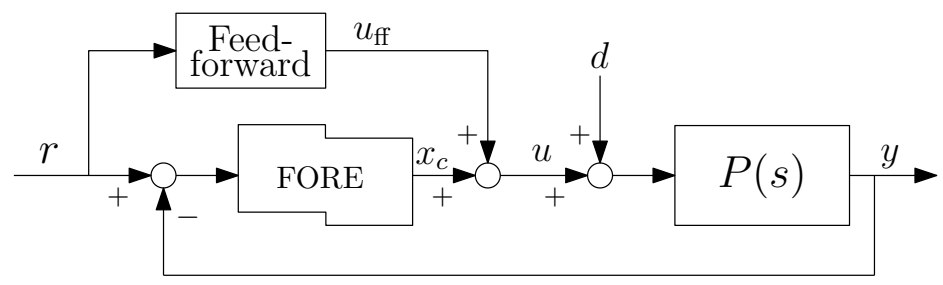

Figure 7.5: Set-point regulation of linear SISO plants using a FORE.

Suppose that the transfer function of the plant (7.11) from $u$ to $y$ does not have zeros at the origin. Then set point regulation of the output $y$ can be achieved from $u$. To this aim, define the following scalar:

$$
F= \begin{cases}-\frac{1}{C_{p} A_{p}^{-1} B_{p u}}, & \text { if } A_{p} \text { is invertible } \\ 0, & \text { otherwise }\end{cases}
$$

corresponding to the inverse of the DC gain of the plant, and define $\hat{F}$ as a nominal value for $F$. Then, if the closed loop between the FORE 7.2 and the plant 7.11 , with $d=0, u=x_{c}$ and $e=-y$ is asymptotically stable, set-point regulation can be achieved by using (7.2) with the following feedback interconnection:

$$
\left\{\begin{array}{l}
u=x_{c}+u_{\mathrm{ff}} \\
e=r-y
\end{array}\right.
$$


which involves a constant feedforward signal $u_{\mathrm{ff}}=\hat{F} r$, where $\hat{F}$ is the best available estimate of $F$. The following statement, whose proof is reported in Nešić et al. 2011] establishes useful set-point regulation properties of the closed loop under the assumption that the closed loop with $r=0$ is asymptotically stable.

Theorem 7.5. (FORE set point stabilizer) Suppose that the transfer function of the plant (7.11) from $u$ to $y$ does not have zeros at the origin and that the origin of the reset control system (7.11), 7.2, 7.14 with $r=0$ and $d=0$ is locally asymptotically stable. Then the interconnection of 77.11$),(7.2)$ with state $\left(x_{p},\left(x_{c}, \tau\right)\right)$ via equation 7.14 is such that:

1. if $F=\hat{F}$, then for any constant reference $r^{*} \in \mathbb{R}$ :

(a) if $d=0$, the unique equilibrium point $x^{*}=\left(x_{p}^{*}, 0\right)$, satisfying $y^{*}=C_{p} x_{p}^{*}=r^{*}$ is globally exponentially stable;

(b) for each $p \in[1,+\infty)$ the system is finite gain $t-\mathcal{L}_{p}$ and $t-\mathcal{L}_{p}$ to $t$ - $\mathcal{L}_{\infty}$ stable from $d$ to $\left(x_{p}-x_{p}^{*}, x_{c}\right)$;

2. if $F \neq \hat{F}$, denoting $\Delta F=\hat{F}-F$, there exist positive constants $k, \ell$ and $\gamma$ such that for any constant reference $r^{*} \in \mathbb{R}$ :

$$
\left|y(t, j)-r^{*}\right| \leq \max \left\{k e^{-\ell t}|\tilde{x}(0,0)|, \gamma\|d\|_{\infty}, \gamma \Delta F r^{*}\right\},
$$

where $\tilde{x}(0,0)=\left(x_{p}(0,0)-x_{p}^{*}, x_{c}(0,0)\right)$.

The construction proposed in Theorem 7.5 and shown in Figure 7.5 generalizes the FORE control system construction to the set-point regulation problem. This generalization is quite intuitive when the plant 7.11 has in it an integrator, or more generally an internal model of the reference, and is actually the case for all situations where FOREs have been used in the literature (see for example the discussion in Beker et al. 2004, Section 4.1]). As a matter of fact in that case $F=0$, the feedforward path in Figure 7.5 disappears and the scheme resembles the typical control scheme in feedback from the set-point tracking error. However, when the plant does not satisfy $\operatorname{det}\left(A_{p}\right)=0$, using $\hat{F}=0$ in Figure 7.5 is no longer effective and can lead to undesirable closed-loop behavior, as shown in the next example. 


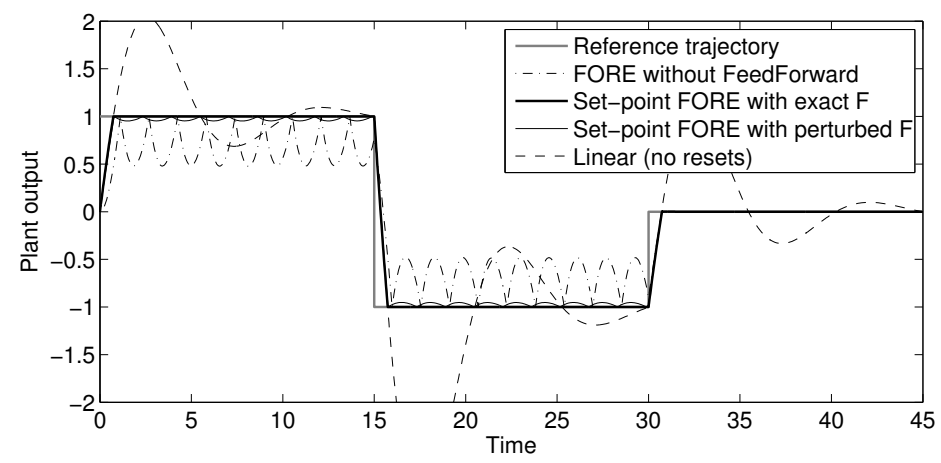

Figure 7.6: Example 7.1 linear response (dashed), FORE without feedforward (dash-dotted), FORE with feedforward and nominal $F$ (bold) and FORE with feedforward and perturbed $F$ (thin solid).

Remark 7.6. Note that applying Theorem 4.6 to system (7.11), (7.2), 7.14 with $r=0$ allows to conclude to $t$-ISS and $t$ - $\mathcal{L}_{p}$ stability from $d$ to $\left(x_{p}, x_{c}\right)$ for any $p \in[1, \infty)$. This property is less relevant here because we are dealing with set-point regulation. Nevertheless, by virtue of Theorem 4.6, it follows directly from the asymptotic stability assumption.

Example 7.1. Consider the plant (7.11) in feedback interconnection (7.14) with the FORE (7.2). Use the following parameters

$$
A_{p}=-1.5, \quad B_{p u}=1, \quad C_{p}=1, \quad b_{c}=2, \quad a_{c}=1,
$$

so that $F=0.75$. In Figure 7.6 , the grey curve represents the reference signal $r$ and the dashed line represents the response of the system without resets and with the feedforward path (using $F=\hat{F}$ ), which is exponentially stable for these parameters. Note that this response is only illustrative of how the reset mechanism changes the underlying linear dynamics but cannot be used to establish any superiority of reset control versus linear solutions. Indeed, many alternative linear control schemes could be considered for this set-point regulation problem, depending on what the performance goal is. The solid line reports the response of the FORE control system implemented without the feedforward path (or, equivalently, by selecting $\hat{F}=0$ ) and the bold line 
reports the response of the FORE control system implemented according to Figure 7.6 with $F=\hat{F}$. Finally, the thin solid curve shows the response when $F$ is increased by $10 \%$ with respect to the correct value. The resulting response is a slight deterioration of the desirable bold response as anticipated by the result at item 2 of Theorem 7.5 .

\subsubsection{Adaptive feedforward injection}

When the plant to be controlled is scalar, namely its dynamics is given by a first order linear filter, it is possible to exploit the fact that after each reset the closed-loop error dynamics starts from zero initial conditions, because the plant output error is crossing zero and the controller state is reset to zero. Moreover, for scalar plants the disturbance $d$ acting on the state equation can be considered without loss of generality as an input matched disturbance acting as follows on the plant equation:

$$
\dot{y}=a_{p} y+b_{p}(u+\bar{d}(r))
$$

where $a_{p}$ is the plant pole and $b_{p}$ is the plant input gain, just as in 7.4. Focusing on plant (7.16), an online adaptation law for the feedforward signal $u_{\mathrm{ff}}$ is proposed, which is constant during flows and is adapted by suitable jumps at resets. Due to this special nature of the adaptation law, the following assumption is required.

Assumption 7.1. The origin of the reset closed-loop (7.16), (7.2) with $e=-y, u=x_{c}$ and $\bar{d}(r)=0$ is exponentially stable. Moreover, the reset closed loop is such that from any nonzero initial condition, there exists a finite time when the response will present a reset.

Remark 7.7. Note that necessary and sufficient conditions for exponential (and asymptotic) stability of the planar reset closed loop in Assumption 7.1 have been given in Theorem 7.1. As for the other requirement in Assumption 7.1, it is automatically satisfied whenever the underlying linear dynamics of the closed loop before resets generates diverging trajectories. Indeed, in that case if no resets occur, trajectories would diverge, thereby contradicting the exponential stability assumption. This is a typical situation in practical cases where a reset controller is used. Indeed, to induce fast transients, the underlying 
linear dynamics is destabilized, and then resets are exploited for stabilization purposes. An example of such a design scheme is given next in Section 8.4 .

For constant values of $r, \bar{d}(r)$ and $u_{\mathrm{ff}}$, define $u_{\mathrm{ff}}^{*}(r)=F r-\bar{d}(r)$ as the unknown steady-state control input required by the set-point regulation scheme. To design an adaptive version of the feedforward signal $u_{\mathrm{ff}}$ in 7.14, define $\tilde{u}_{\mathrm{ff}}:=u_{\mathrm{ff}}-u_{\mathrm{ff}}^{*}(r)$ and, without loss of generality let us address the case $\tilde{u}_{\mathrm{ff}}>0$. Consider also the change of coordinates $\left(x_{c}, \tilde{y}\right)=\left(x_{c}, y-r\right)$ and notice that the closed-loop system can be written in the new coordinates as

$$
\begin{aligned}
{\left[\begin{array}{c}
\dot{\tilde{y}} \\
\dot{x}_{c}
\end{array}\right] } & =\left[\begin{array}{cc}
a_{p} & b_{p} \\
-b_{c} & a_{c}
\end{array}\right]\left[\begin{array}{c}
\tilde{y} \\
x_{c}
\end{array}\right]+\left[\begin{array}{c}
b_{p} \\
0
\end{array}\right] \tilde{u}_{\mathrm{ff}} \\
& =: A_{c l}\left[\begin{array}{c}
\tilde{y} \\
x_{c}
\end{array}\right]+B_{c l} \tilde{u}_{\mathrm{ff}} .
\end{aligned}
$$

It can be proven (see [Panni et al. 2014, Lemma 1]) that, as long as $u_{\mathrm{ff}}$ is constant inbetween resets, the FORE state $x_{c}$ just before a resetting action is proportional to the feedforward mismatch:

$$
x_{c}(T, 0)=-M_{x}\left(u_{\mathrm{ff}}-u_{\mathrm{ff}}^{*}(r)\right) .
$$

Remark 7.8. Assumption 7.1 and the strong requirement that the plant is scalar are two key properties to be able to prove the monotonicity in (7.18). Indeed, if Assumption 7.1 does not hold, then there can be defective cases where the error dynamics in 7.17$)$ converges to a nonzero value of $\tilde{y}$ without any reset (even though an $t$ - $\mathcal{L}_{\infty}$ bound on the response will hold because of exponential stability and the $t$-ISS properties established in Theorem 7.5. An example showing the relevance of Assumption 7.1 is obtained with the parameters $\left[\begin{array}{ll}a_{p} & b_{p} \\ b_{c} & a_{c}\end{array}\right]=\left[\begin{array}{cc}-3 & 1 \\ 1 & -1\end{array}\right]$, which lead to an exponentially converging response that never resets (it can be checked, for example, running a simulation with $\bar{d}=0$, $\left(y(0,0), x_{c}(0,0)\right)=0$ and some nonzero constant reference $\left.r\right)$.

Also the requirement that the plant is a scalar filter is necessary, in general, to prove the monotonic relation 7.18 . For example, using $a_{c}=$ $3, b_{c}=1$ and an exponentially stable plant with two poles in $\{-1,-2\}$ and no zeros generates a response where the sign of $y$ (therefore, also 
that of $x_{c}$ ) switches between positive and negative and vice-versa for each consecutive pairs of resets. Therefore, the state $x_{c}(t, j)$ before a reset does not anymore carry the information on the sign of the feedforward mismatch $\tilde{u}_{\mathrm{ff}}>0$, and more sophisticated proof techniques, should be adopted or more sophisticated adaptation laws should be used. A similar undesirable result is also obtained for a relative degree one stable minimum phase plant having a zero in -80 and poles in $\{-3,-2\}$, loop gain $b_{c}=1$ and FORE pole $a_{c}=1$.

Practical experience suggests that even for these higher order plants the adaptation law proposed here successfully converges to the correct feedforward term, however the convergence is characterized by a peculiar oscillatory behavior of the feedforward mismatch $\tilde{u}_{\mathrm{ff}}>0$, which suggests that a Lyapunov proof of its convergence cannot be obtained using the convenient path involving the monotonicity property in (7.18).

To obtain an adaptive version of the feedforward term in Figure 7.5. we augment the FORE dynamics 7.2 with an extra state $u_{\mathrm{ff}}$ governed by the following hybrid dynamics:

$$
\left\{\begin{array}{rlrl}
\dot{u}_{\mathrm{ff}}=0, & & \left(e, x_{c}\right) \in \mathcal{F} \text { or } \tau \in[0, \rho], \\
u_{\mathrm{ff}}^{+}=u_{\mathrm{ff}}+\lambda x_{c}, & \left(e, x_{c}\right) \in \mathcal{J} \text { and } \tau \in[\rho, 2 \rho],
\end{array}\right.
$$

where we have left unchanged the flow and jump sets of $(7.2)$ and the scalar $\lambda>0$ should be selected sufficiently small, according to the next result whose proof is given in Panni et al. 2014.

Proposition 7.1. Consider a scalar plant 7.16 in feedback interconnection 7.14 with the adaptive reset controller (7.2), 7.19). Under Assumption 7.1, there exists a small enough $\rho^{*}$ and a small enough $\lambda^{*}$ such that for all $\rho \in\left(0, \rho^{*}\right)$, all $\lambda \in\left(0, \lambda^{*}\right)$, and any constant pair $(r, \bar{d}(r))$, the state $u_{\mathrm{ff}}$ exponentially converges to $u_{\mathrm{ff}}^{*}(r)$ and the plant output $y$ exponentially converges to $r$.

Remark 7.9. Useful insight can be given for tuning the parameter $\lambda$ in the adaptation law of the controller (7.19). In particular, a useful experimental strategy is that of increasing $\lambda$ until the stability limit (typically, oscillatory behavior will be experienced for values of $\lambda$ around $\lambda^{*}$ ). 
Then, once the critical value $\lambda^{*}$ has been determined, a recommended strategy is to select $\lambda$ as half of that value. Indeed, some nontrivial calculations exploiting relation 7.18 show that choosing $\lambda=\frac{\lambda^{*}}{2}$ results in an equivalent dead beat discrete dynamics for $\tilde{u}_{\mathrm{ff}}$. The expected outcome is that, regardless of the value of $r$, the steady-state value of $u_{\mathrm{ff}}$ is quickly determined after the first reset.

\subsubsection{Parametrization of a position-dependent input bias}

The rationale behind the adaptive reset controller 7.19 ) is that in a setpoint regulation scheme with a piecewise constant reference $r$, whenever a step reference change is experienced, the feedback action provided by the FORE state $x_{c}$ will stabilize the error dynamics, while the adaptive feedforward action given by the FORE state $u_{\mathrm{ff}}$ will converge to the steady-state input required for asymptotic regulation.

For improved performance, it is desirable to implement the adaptive reset controller in such a way that, whenever the same reference is repeated, the adaptation carried out at the previous instance of the same reference value is exploited to speed up the convergence of $u_{\mathrm{ff}}$ to the correct value $u_{\mathrm{ff}}^{*}(r)=F r-\bar{d}(r)$. This intuitive goal can be accomplished by suitably parametrizing such a correct value by $u_{\mathrm{ff}}^{*}(r)=$ $\sum_{i=1}^{N} \alpha_{i}^{*} \psi_{i}(r)$, which is the optimal value of the following parametric equation for $u_{\mathrm{ff}}$ :

$$
u_{\mathrm{ff}}=\sum_{i=1}^{N} \alpha_{i} \psi_{i}(r)=\Psi^{\top}(r) \alpha .
$$

In 7.20 , the vector $\Psi(r) \in \mathbb{R}^{N}$ gathers together $N$ constant independent basis functions $\psi_{i}(r)$ and $\alpha=\left[\begin{array}{ccc}\alpha_{1} & \cdots & \alpha_{N}\end{array}\right]^{\top}$ contains the parameters to be adapted. With the parametrization $\sqrt{7.20}$, it is then convenient to replace the state $u_{\mathrm{ff}}$ in the adaptive FORE (7.2), (7.19) by a new state $\alpha$ whose hybrid dynamics is given by:

$$
\left\{\begin{aligned}
\dot{\alpha} & =0, & \left(v, x_{c}\right) & \in \mathcal{F} \text { or } \tau \in[0, \rho], \\
\alpha^{+} & =\alpha+\lambda \frac{\Psi(r)}{\Psi^{\top}(r) \Psi(r)} x_{c}, & & \left(v, x_{c}\right) \in \mathcal{J} \text { and } \tau \in[\rho, 2 \rho],
\end{aligned}\right.
$$

Then, for each fixed value of $r$, the parametrized adaptive FORE (7.2), (7.21) preserves the convenient features of the original 
scheme (7.2), 7.19) established in Proposition 7.1. Indeed, using the parametrization (7.20), the jump rule in (7.21) corresponds to the following update rule for the feedforward term:

$$
u_{\mathrm{ff}}^{+}=\Psi^{\top}(r) \alpha^{+}=\Psi^{\top}(r) \alpha+\Psi^{\top}(r) \lambda \frac{\Psi(r)}{\Psi^{\top}(r) \Psi(r)} x_{c}=u_{\mathrm{ff}}+\lambda x_{c},
$$

which coincides with the one in 7.19 .

The advantage of the implementation (7.21) as compared to the previous one, is that under the assumption that there exists $\alpha^{*}$ such that $u_{\mathrm{ff}}^{*}(r)=\Psi^{\top}(r) \alpha^{*}$, as long as the adaptive system is persistently excited, the state $\alpha$ will converge to $\alpha^{*}$ and the closed-loop responses will be increasingly faster because upon the occurrence of a new step reference $r$, the adaptation will already start from a value $u_{\mathrm{ff}}$ coinciding with (or close to) the asymptotic value $u_{\mathrm{ff}}^{*}(r)$. We do not pursue a formal analysis of these properties which would require addressing a number of technicalities, but similar reasonings to those of the proof of Proposition 7.1 can be carried out by replacing the Lyapunov term $\left|\tilde{u}_{\mathrm{ff}}\right|^{2}$ by $|\tilde{\alpha}|^{2}$, where $\tilde{\alpha}:=\alpha-\alpha^{*}$. In particular, using the update rule in (7.21), relation (7.18) and a small enough $\lambda$ satisfying $\lambda M_{x}<2$, the change of this function across jumps corresponds to

$$
\begin{aligned}
\left|\tilde{\alpha}^{+}\right|^{2}-|\tilde{\alpha}|^{2}= & \left|\alpha+\lambda \frac{\Psi(r)}{\Psi^{\top}(r) \Psi(r)} x_{c}-\alpha^{*}\right|^{2}-\left|\alpha-\alpha^{*}\right|^{2} \\
= & \left|\tilde{\alpha}-\lambda M_{x} \frac{\Psi(r) \Psi^{\top}(r)}{\Psi^{\top}(r) \Psi(r)} \tilde{\alpha}\right|^{2}-|\tilde{\alpha}|^{2} \\
= & \tilde{\alpha}^{\top}\left(I-2 \lambda M_{x} \frac{\Psi(r) \Psi^{\top}(r)}{\Psi^{\top}(r) \Psi(r)}\right. \\
& \left.\quad+\lambda^{2} M_{x}^{2} \frac{\Psi(r) \Psi^{\top}(r) \Psi(r) \Psi^{\top}(r)}{\left(\Psi^{\top}(r) \Psi(r)\right)^{2}}\right) \tilde{\alpha}-\tilde{\alpha}^{\top} \tilde{\alpha} \\
= & -\lambda M_{x}\left(2-\lambda M_{x}\right) \tilde{\alpha}^{\top} \frac{\Psi(r) \Psi^{\top}(r)}{\Psi^{\top}(r) \Psi(r)} \tilde{\alpha} \leq 0,
\end{aligned}
$$

which guarantees that the norm of the parameters estimation error $\tilde{\alpha}$ is nonincreasing.

Establishing a strict decrease of $|\tilde{\alpha}|$ from the above Lyapunov inequalities can be done by requiring some kind of persistence of ex- 
citation from the piecewise constant reference signal $r$. In particular, following discrete-time derivations parallel to the continuous-time results in [Khalil, 2002, Theorem 8.5], we need to require the existence of two positive numbers $\epsilon \in \mathbb{R}_{\geq 0}$ and $M \in \mathbb{Z}_{\geq 0}$ such that, denoting by $\left\{t_{i}\right\}_{i \geq 0}$ the ordinary times when the jumps occur,

$$
\sum_{i=k}^{k+M} \frac{\Psi\left(r\left(t_{i}\right)\right) \Psi^{\top}\left(r\left(t_{i}\right)\right)}{\Psi^{\top}\left(r\left(t_{i}\right)\right)^{\top} \Psi\left(r\left(t_{i}\right)\right)} \geq \epsilon I, \quad \forall k \in \mathbb{Z}_{\geq 0} .
$$

A proof of the convergence of $\tilde{\alpha}$ to zero should also involve addressing the problem of defective isolated update laws happening after a step change of the reference. Indeed, a step change in $r$ causes a different change of coordinates of the error dynamics, resulting in a different initial condition, so that condition $(7.18$ fails to hold at the first reset. Due to this reason, the adaptation of $\alpha$ should be inhibited at the first reset after any step reference change of $r$. As customary in the adaptive control context, a possible robust implementation of this mechanism can be carried out by generating a high-pass filtered version $r_{f}$ of the reference $r$ :

$$
r_{f}(s)=\frac{s}{s+\lambda_{h}} r(s)
$$

and then pushing to zero the adaptation gain $\lambda$ if the norm of $r_{f}$ is larger than a threshold $\bar{r}_{f}$, replacing $(7.21)$ by:

$$
\begin{aligned}
\dot{\alpha}= & 0, \quad\left(v, x_{c}\right) \in \mathcal{F} \text { or } \tau \in[0, \rho], \\
\alpha^{+}= & \alpha+\lambda \max \left\{0, \min \left\{1, \quad\left(v, x_{c}\right) \in \mathcal{J} \text { and } \tau \in[\rho, 2 \rho],\right.\right. \\
& \left.\left.\left|\bar{r}_{f}-r_{f}\right|\right\}\right\} \frac{\Psi(r)}{\Psi^{\top}(r) \Psi(r)} x_{c},
\end{aligned}
$$

Clearly, with the mechanism above, the adaptation is only enabled as long as the piecewise constant reference dwells on each constant value for a long enough time. Moreover, this mechanism works also whenever the reference signal $r$ is affected by noise (possibly because it comes from a higher level control system) as long as the threshold $\bar{r}_{f}$ is not too small.

In Chapter 8 this control strategy is illustrated on the experimental control of an EGR valve of a Diesel engine. 


\subsection{Reference tracking}

The set-point regulation scheme presented in the previous section is not suitable for tracking a generic time-varying reference. This fact can be appreciated, for example, in the undesirable sinusoidal tracking experiments reported later in Section 8.6. Based on the results in Cordioli et al. [2015], we generalize here the scheme with the goal in mind of tracking a generic time-varying but continuously differentiable reference $r$ under the strong assumption that both $r$ and its time derivative $\dot{r}$ are available for building the adaptive feedforward input $u_{\mathrm{ff}}$. Then the scheme of Figure 7.5 naturally extends to the generalization represented in Figure 7.7 .

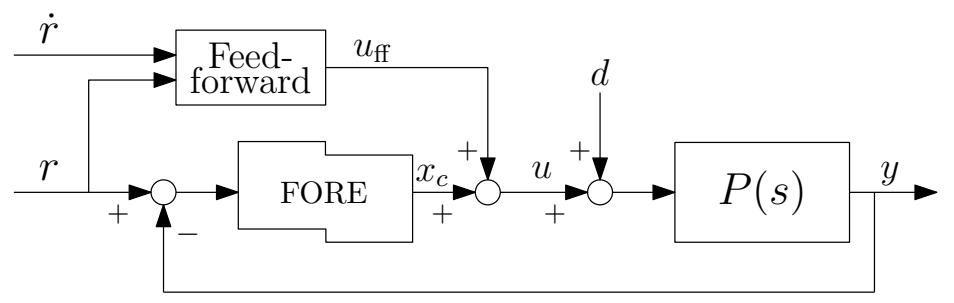

Figure 7.7: FORE-based reference tracking scheme with adaptive feedforward.

Following classical result on linear and nonlinear regulation theory, while $u_{\mathrm{ff}}$ will take care of generating the steady-state input, the regulation error will be driven to zero by the action of the FORE feedback stabilizer.

In particular, inspired by the adaptation law presented in Section 7.4 we propose the following parametrization of the feedforward signal $u_{\mathrm{ff}}$ in 7.14 :

$$
u_{\mathrm{ff}}=\Psi^{\top}(r, \dot{r}) \alpha:=\left[\begin{array}{l}
1 \\
r \\
\dot{r}
\end{array}\right]^{\top}\left[\begin{array}{l}
\alpha_{1} \\
\alpha_{2} \\
\alpha_{3}
\end{array}\right],
$$

where the parameters $\alpha$ can be adapted online and the vector $\Psi$ is fixed

a priori and changes according to the variation of the smooth reference $r$.

With the parametrization in 7.23 and the dynamics 7.16$), 7.2$, 
7.14, there exists $\alpha^{*}$ such that from suitable initial conditions, the open-loop control signal:

$$
u_{\mathrm{ff}}^{*}=\Psi^{\top}(r, \dot{r}) \alpha^{*}
$$

guarantees perfect tracking, that is $y(t)=r(t)$ for all $t \geq 0$. To see this, select

$$
\alpha^{*}=\left[\alpha_{1}^{*} \alpha_{2}^{*} \alpha_{3}^{*}\right]^{\top}=\frac{1}{b_{p}}\left[\bar{d}-a_{p} 1\right]^{\top}
$$

and notice that, using (7.16), one gets:

$$
\begin{aligned}
\dot{e} & =\dot{r}-\dot{y}=\dot{r}-a_{p} y-b_{p} u_{\mathrm{ff}}^{*}-\bar{d} \\
& =\dot{r}-a_{p}(r-e)-\left(\bar{d}-a_{p} r+\dot{r}\right)+\bar{d}, \\
& =a_{p} e,
\end{aligned}
$$

which implies that $e(t)=0$ for all $t$ as long as $e(0)=0$. Note also that the error dynamics is not necessarily exponentially stable (this is why the action of a feedback stabilizer is necessary in the hybrid control scheme).

With this parametrization, we can follow similar steps to those in Section 7.4 and maintain the parameters $\alpha$ constant during flow, while adapting them at jumps (resets). However, the adaptation must be more sophisticated than that in 7.21 because now the reference is time-varying. Following the derivations in Cordioli et al. 2015 an effective solution exploting the information stored in the FORE state $x_{c}$ just before a jump corresponds to the following dynamics:

$$
\begin{array}{ll}
\dot{\alpha}=0, & \left(e, x_{c}\right) \in \mathcal{F} \text { or } \tau \in[0, \rho], \\
\alpha^{+}=\alpha+\lambda \frac{\varphi(\tau)}{\max \left\{\eta,|\varphi(\tau)|^{2}\right\}} \Psi(r, \dot{r}) x_{c}, & \left(e, x_{c}\right) \in \mathcal{J} \text { and } \tau \in[\rho, 2 \rho],
\end{array}
$$

with

$$
\varphi(\tau):=\int_{0}^{\tau} C e^{A_{f}(\tau-s)} B_{f} d s
$$

where matrices $A_{f}$ and $B_{f}$ arise from the continuous dynamics and correspond to

$$
\left[A_{f} \mid B_{f}\right]=\left[\begin{array}{cc|c}
a_{p} & -b_{p} & -b_{p} \\
b_{c} & a_{c} & 0
\end{array}\right]
$$


Moreover, $\eta>0$ is a (typically small) parameter ensuring that the adaptation does not blow up when little information is provided by $x_{c}$, and where, just as before, the scalar gain $\lambda>0$ should be selected sufficiently small.

In Chapter 9, this control strategy will be illustrated on the experimental control of an electromechanical valve adopted in a power-split transmission system. 
8

\section{FORE control of an EGR valve}

\subsection{Overview}

Exhaust gas recirculation (EGR) is one of the most effective technique to reduce emissions in diesel engines. It works by recirculating a small amount of exhaust gas in the intake manifold, which contains the fresh air that is collected to perform the combustion. This is done by placing a valve between the intake manifold and the exhaust manifold. There are two control stages for the EGR valve: a higher level control system commands a reference position for the valve in order to regulate the amount of the exhaust gas to be recirculated, and a lower level one regulates the actuator position to the desired reference value. In this section we illustrate an application of adaptive FORE control to the lower level stage of an EGR valve. Part of these results are reported in Panni et al. 2014.

The main control goal is to provide a fast set-point regulation of the EGR valve position and rejection of a constant disturbance acting as a load force on the valve actuator. In the real engine application, this disturbance comes from the force generated by the pressure difference between the intake and the exhaust manifold. In a laboratory experiment, described below, the spring which is embedded into the 
valve and an elastic band are used to represent the load disturbance.

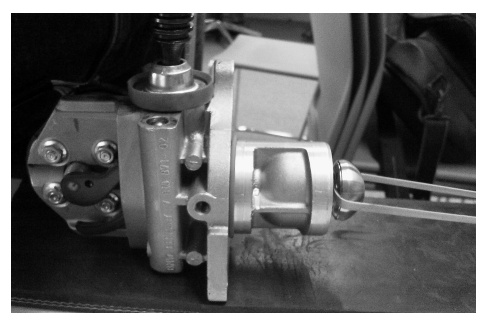

Figure 8.1: A snapshot of the laboratory experiment with the EGR valve.

\subsection{Model parameters identification}

Figure 8.1 is a snapshot of the EGR valve used for a laboratory experiment, which is clamped to a table using a vice. It is possible to distinguish between the mechanical assembly which contains the valve itself and the electric brushless DC motor that drives the valve. The DC motor is a torque motor characterized by a maximum rotation of 75 degrees, a maximum torque of $540 \mathrm{mNm}$ and a response time smaller than $50 \mathrm{~ms}$. On the side of the motor, a spring keeps the valve closed when the motor is unpowered. A cam mechanism converts the angular rotation of the motor into the linear displacement of the valve, within a limited segment. An embedded sensor provides a voltage output proportional to the linear position of the valve. An input voltage is used to provide the power signal to the DC motor. The feedback control system is designed with MATLAB/Simulink and implemented on a dSpace real-time rapid prototyping system. In particular, on the measurement side, the output voltage providing information about the valve position is converted into the percentage of the position range $[0,100]$, where 0 means that the valve is fully closed and 100 means that the valve is fully open. On the actuation side, an analog output of the MATLAB/dSpace system is connected to a MOSFET-based commercial PWM power amplifier, which is based on an $\mathrm{H}$ bridge structure, supports a 5.5 to $30 \mathrm{~V}$ voltage range and can deliver a maximum continuous current of $15 \mathrm{~A}$. The PWM amplifier provides a $35 \mathrm{kHz} \mathrm{PWM}$ voltage signal switching between $0 \mathrm{~V}$ and $14 \mathrm{~V}$ or between $-14 \mathrm{~V}$ and 
$0 V$ depending on the state of the H-bridge. The duty cycle can be conveniently adjusted using a reference voltage ranging between $0 \mathrm{~V}$ and $5 \mathrm{~V}$ and the state of the H-bridge is controlled using a $5 \mathrm{~V}$ digital signal. Those reference voltages are provided by suitable outputs of the MATLAB/dSpace system, whose values are commanded on the software side by a suitable Simulink block ranging from -1 (negative signal with maximum duty cycle, namely maximum negative average voltage) to +1 (positive signal with maximum duty cycle, namely maximum positive average voltage).
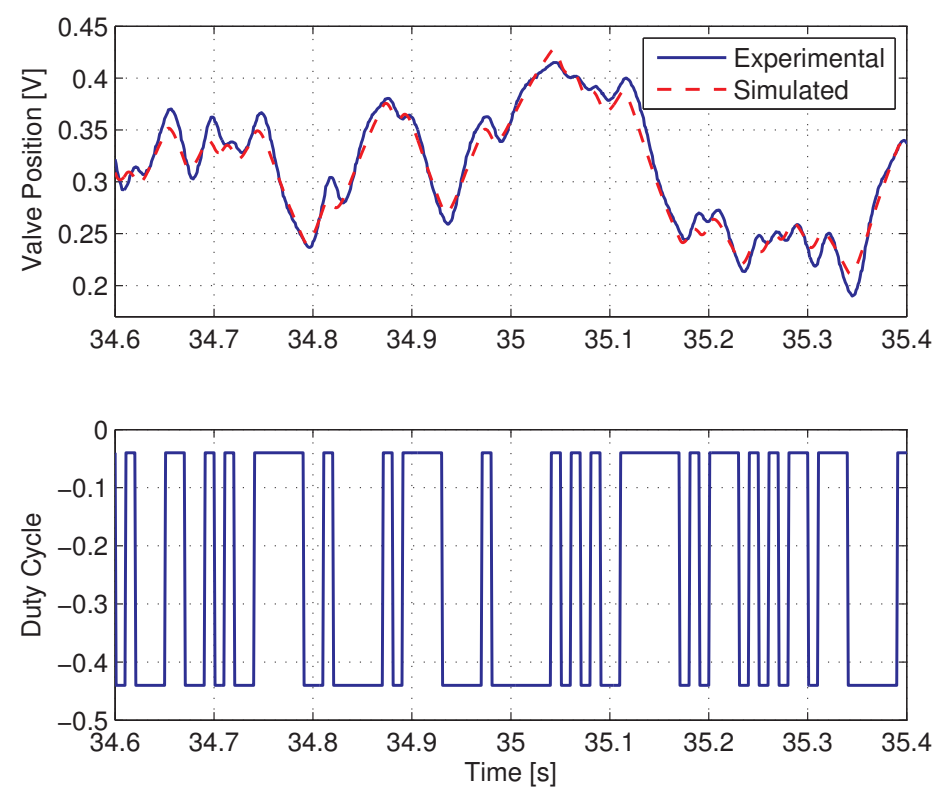

Figure 8.2: Simulated and experimental responses using the model 8.1.

The identification of the valve transfer function from the duty cycle input to the voltage (position) output was carried out using a PseudoRandom Binary Sequence (PBRS) input signal. Figure 8.2 shows a portion of the data used for the identification process (solid lines in the upper and lower plots) together with the simulated output arising from the identified transfer function 8.1 reported below. The mean value of the PRBS was chosen to compensate for the influence of the spring. In this way, if the maximum frequency of the PRBS is sufficiently high, the 
valve roughly oscillates around the middle position without impacting the limiters.

A model has been estimated with the MATLAB System Identification Toolbox (Ljung [2007]). Good results were obtained estimating continuous-time process models. The raw input/output data of a 80 seconds experiment was used for the identification phase, while another set of 30 seconds of data was used for validation, giving comparable results to the ones of the upper trace in Figure 8.2. A two poles transfer function gave a $78 \%$ fit to the measured data, using

$$
P(s)=\frac{2200}{(s+164.4)(s+10.69)}
$$

Adding extra dynamics does not improve noticeably the fitting of the estimated model. Hence, the transfer function (8.1) was chosen as a model for the EGR valve. This model is also reasonable from a physical point of view. Indeed, neglecting the electrical time constant of the valve, the system is well represented by a second order mass-springdamper system. Note that the electrical time constant is neglected in this model, nevertheless, a more accurate description of the valve would indeed require third order dynamics, as confirmed by the open-loop time-optimal input signal discussed in Section 8.7 (see also Figure 8.9), which switches three times.

Experiments show that the influence of the stiction is relevant. In particular, imposing low but considerable values of the control input when the valve is still, does not move the valve. Of course, the linear transfer function cannot capture this effect. Nevertheless, the proposed control scheme is aggressive enough to remain essentially unaffected by the undesired stiction effect. The effectiveness of the design technique proposed in the next section does not rely on a precise model, as it only relies on certain properties of the transfer function. However, the identified model is accurate enough to allow for the closed-loop performance assessment in simulation, before the experimental tests. 


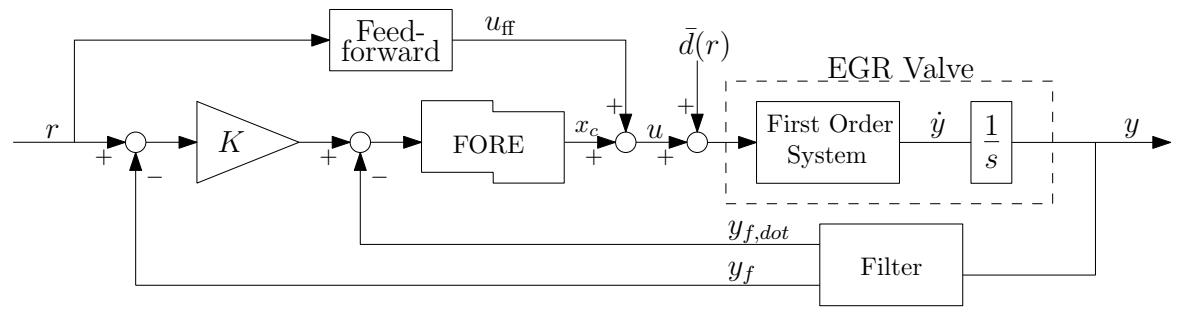

Figure 8.3: Block diagram of the double-loop control scheme.

\subsection{Proposed regulation scheme}

The EGR valve transfer function (8.1) identified in Section 8.2 has two poles so that the control strategy presented in Section 7.4 cannot be directly applied. To overcome this issue, we employ the double loop scheme shown in Figure 8.3 where a linear dynamical filter is connected to the EGR position output to provide an estimate $\dot{y}_{f}$ of the EGR valve speed according to the results in Nicosia et al. [1990] (alternative observer designs for plants with unknown inputs could also be employed). Then, as shown in the block diagram, an inner loop in feedback from the estimate $\dot{y}_{f}$ of $\dot{y}$ can be implemented according to the technique in Section 7.4. The scheme in feedback from $\dot{y}_{f}$ is a sufficiently accurate approximation of the scheme in feedback from $\dot{y}$ as long as the filter parameters are fast enough. Finally, an outer loop regulates the position by way of the static gain $K$. Note that by writing the EGR valve model as in Figure 8.3 we ignore the effect of the spring and friction acting on the valve and assume that the slow pole in (8.1) is at zero. This is reasonable if one considers that the disturbance $\bar{d}(r)$ can capture the steady-state effects of these neglected terms. The advantage of the arising scheme of Figure 8.3 is twofold: 1) the scheme guarantees zero steady-state error because the integrator serves as an internal model of the reference which would otherwise be missing given the static nature of the outer loop; 2) the scheme is independent of the specific model and it appears to be more suited for the industrial application under consideration where the valve parameters may be subject to variation due to construction issues and aging.

According to the results in Nicosia et al. [1990, the filter in Fig- 
ure 8.3 corresponds to the following high-gain observer dynamics:

$$
\begin{aligned}
\dot{y}_{f} & =y_{f, d o t}+\frac{1}{\epsilon} h_{p}\left(y-y_{f}\right) \\
\dot{y}_{f, d o t} & =\frac{1}{\epsilon^{2}} h_{v}\left(y-y_{f}\right)
\end{aligned}
$$

which provides an output $y_{f}$ corresponding to a filtered version of the valve position $y$ and an output $y_{f, d o t}$ corresponding to a filtered version of its time derivative $\dot{y}$ (see Nicosia et al. [1990 for details). The filter parameters are chosen as $\left(h_{p}, h_{v}\right)=(5.0,6.0)$ so that the roots of the characteristic polynomial $p(\lambda)=\lambda^{2}+\lambda h_{p}+h_{v}$ have negative real part. The parameter $\epsilon$ is a tuning knob: large values of $\epsilon$ correspond to a very slow filter and small values of $\epsilon$ correspond to a fast filter. The main advantage of this filter is that the tuning is easily carried out by adjusting the scalar $\epsilon$.

Regarding the tuning of $\epsilon$, the filter has to be much faster than the speed of the reset control loop, so that its dynamics is negligible from the point of view of the scheme of Section 7.4. Nevertheless, the position signal is affected by a certain amount of measurement noise. For this reason the filter cannot be too fast and this limits the performance of the system. The tuning of $\epsilon$ then comprises a trade-off between the two aspects highlighted above and the value selected for our experiments is $\epsilon=0.001$.

\subsection{Results with adaptive feedforward}

A first set of experiments was carried out on the laboratory equipment described in Section 8.2 by employing the FORE control system with feedforward term 7.2, (7.14), 7.19) described in Section 7.4.2 within the scheme of Figure 8.3 . Once the filter parameters discussed in the previous section were fixed, the FORE parameters have been selected according to the results in Theorem 7.1, stating that a large enough loop gain $b_{c}$ and/or a large enough FORE pole $a_{c}$ suffice to induce closed-loop exponential stability. In particular, a large value of $a_{c}$ has been preferred because it leads to very fast transients comprising exponentially unstable branches stabilized by resets (in this way Assumption 7.1 is satisfied). Note that even though Theorem 7.1 states that 
arbitrarily large $a_{c}$ and $b_{c}$ can be employed, the effect of the sampleand-hold device, delays in the control loop and the presence of the filter (8.2) impose a limit on the parameters. In light of the above considerations, the parameters have been chosen as $a_{c}=60, b_{c}=0.013$, while the outer loop gain of Figure 8.3 has been selected as $K=140$ to induce a fast stabilization with little overshoot.
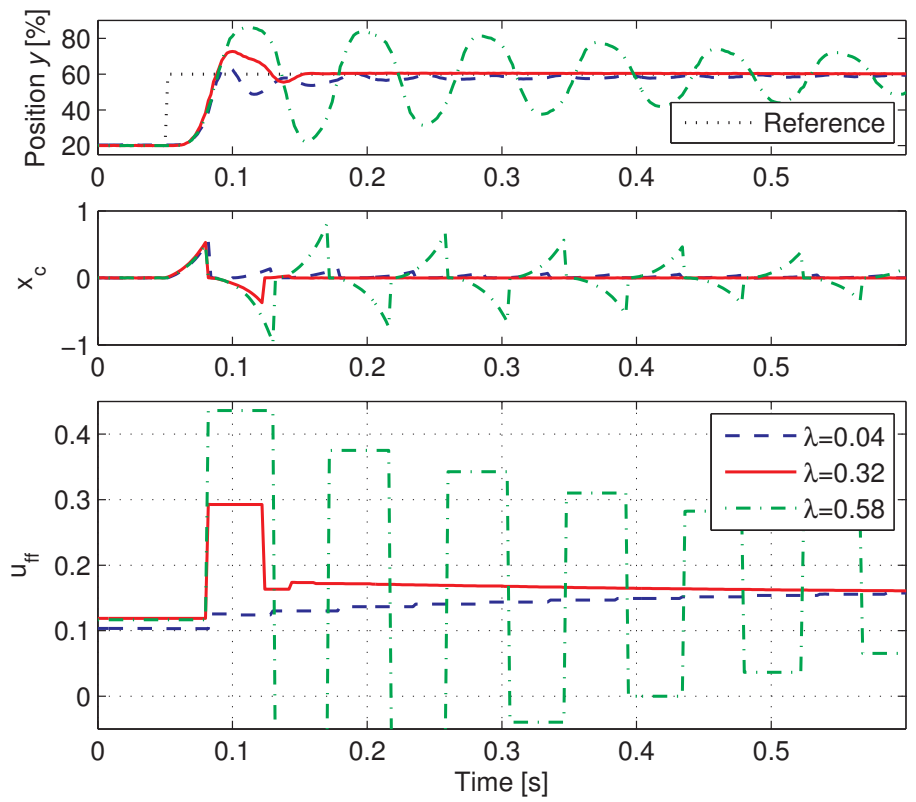

Figure 8.4: Experimental step responses with several values of $\lambda$.

Regarding the selection of $\lambda$, according to the tuning technique proposed in Remark 7.9, $\lambda$ was increased up to the value where oscillations of $u_{\mathrm{ff}}$ are experienced (this corresponds to $\lambda^{*}=0.64$ ). Then the deadbeat value was selected as half of $\lambda^{*}$, that is $\lambda=0.32$. The relevance of this tuning strategy is illustrated in Figure 8.4 where several experimental step responses from $20 \%$ to $60 \%$ of the valve position are reported:

1. with the optimal value of $\lambda=0.32$ leading almost to a dead-beat response of $u_{\mathrm{ff}}$ (red solid); 
2. with the value $\lambda=0.04$, slightly above zero, leading to a very lightly damped convergence (blue dashed) corresponding to a value of $1-\lambda M_{x}$ slightly smaller than 1 ;

3. with the value $\lambda=0.58$, slightly below the limit $\lambda^{*}$, leading to a very oscillatory response (green dashed-dotted) corresponding to a value of $1-\lambda M_{x}$ slightly greater than -1 .
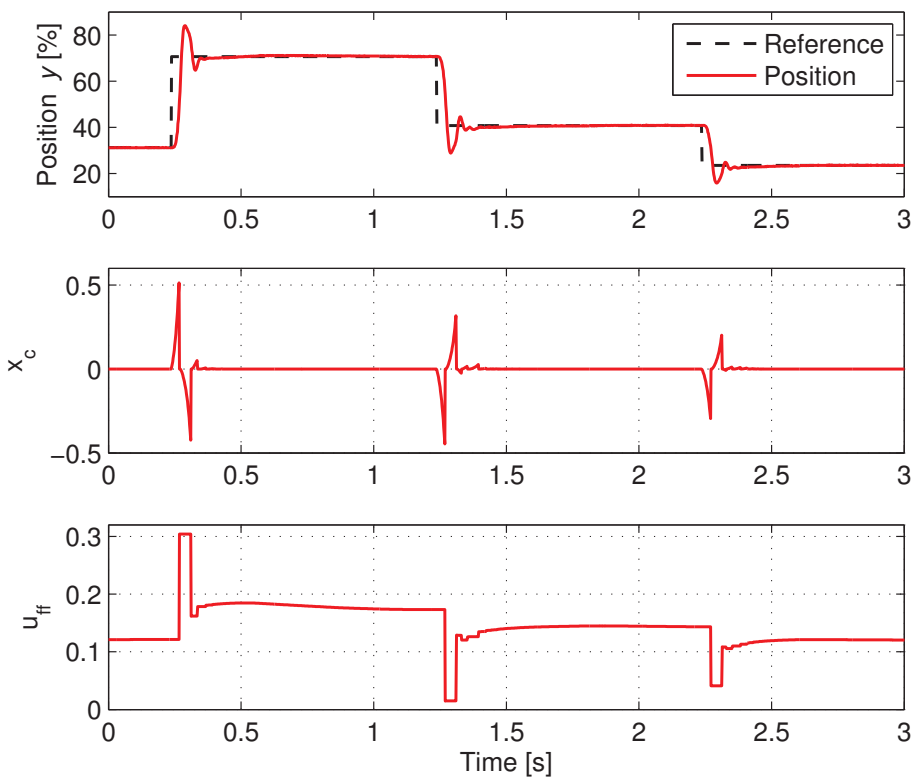

Figure 8.5: A sequence of three step responses when using $\lambda=0.35$.

Figure 8.5 shows that the asymptotic value of the adaptation changes upon any reference change. When focusing on the red response of item 1 above, even though $\lambda$ is tuned to have an almost dead-beat response of $u_{\mathrm{ff}}$, the estimation affects the transient of the step response and reduces the performance essentially causing a significant overshoot (which can be appreciated in the red solid curve in the upper plot of Figure 8.4 and in the red solid curve in the upper plot of Figure 8.5. Moreover, from both Figure 8.4 and 8.5 it appears that the update action for $u_{\mathrm{ff}}$ immediately after the step occurrence induces an undesired 
transient on the trace of $u_{\mathrm{ff}}$ which is only dead-beat after the second reset. This essentially comes from the fact that equation (7.18) only holds if the plant state starts from zero in the transformed coordinates $\left(\tilde{y}, x_{c}\right)$, so while the convergence result of Proposition 7.1 holds, the nonzero initial condition of $\tilde{y}$ before the first reset induces an additional undesired transient. This peculiar behavior motivates the inhibition mechanism introduced in 7.22 and adopted in the next section.

\subsection{Results with parametrized feedforward}

To reduce the undesired overshoot of the step response discussed in the previous section (see, for example, the red solid curve in the the upper plot of Figure 8.4, the FORE control scheme with parametrized feedforward presented in Section 7.4.3 has been experimentally implemented. With this scheme in place, a recurring value of the reference is expected to lead to an increasingly improved performance because the adaptation system takes advantage of the knowledge coming from the past adaptations of $u_{\mathrm{ff}}$ to jump (very close) to the correct value of the feedforward term.

In our laboratory experiment we implemented the FORE control system (7.14), 7.2, 7.21) with a suitable parametrization of $u_{\mathrm{ff}}(r)$ according to 7.20 . In particular, to select the basis functions $\psi_{i}(r)$ leading to a good parametrization of $u_{\mathrm{ff}}(r)$ in 7.20 we collected several steady-state values of $u_{\mathrm{ff}}$ and $r$ using the controller of the previous section. The values of $u_{\mathrm{ff}}$ were collected 5 seconds after each step reference change while the values of the reference was updated randomly in the range $5 \% \div 85 \%$ at each step change. The blue "*" dots in Figure 8.6 represent all the collected steady-state $\left(r, u_{\mathrm{ff}}\right)$ pairs. Their peculiar shape suggests that the correct feedforward term $u_{\mathrm{ff}}^{*}(r)$ is well approximated by a quadratic function of the reference. Note that the spread of the samples in Figure 8.6 is essentially caused by the significant stiction affecting the commercial EGR valve. Based on Figure 8.6.

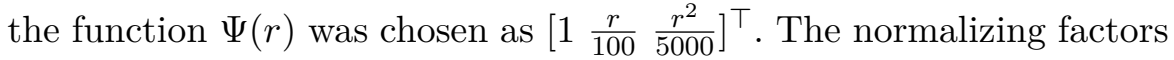
100 and 5000 where introduced to overcome numerical problems of the estimation algorithm. In particular, they where chosen in such a way 
that the elements of $\Psi(r)$ have comparable orders of magnitude in the range $r \in[0,100]$. The red curve in Figure 8.6 represents the esti-

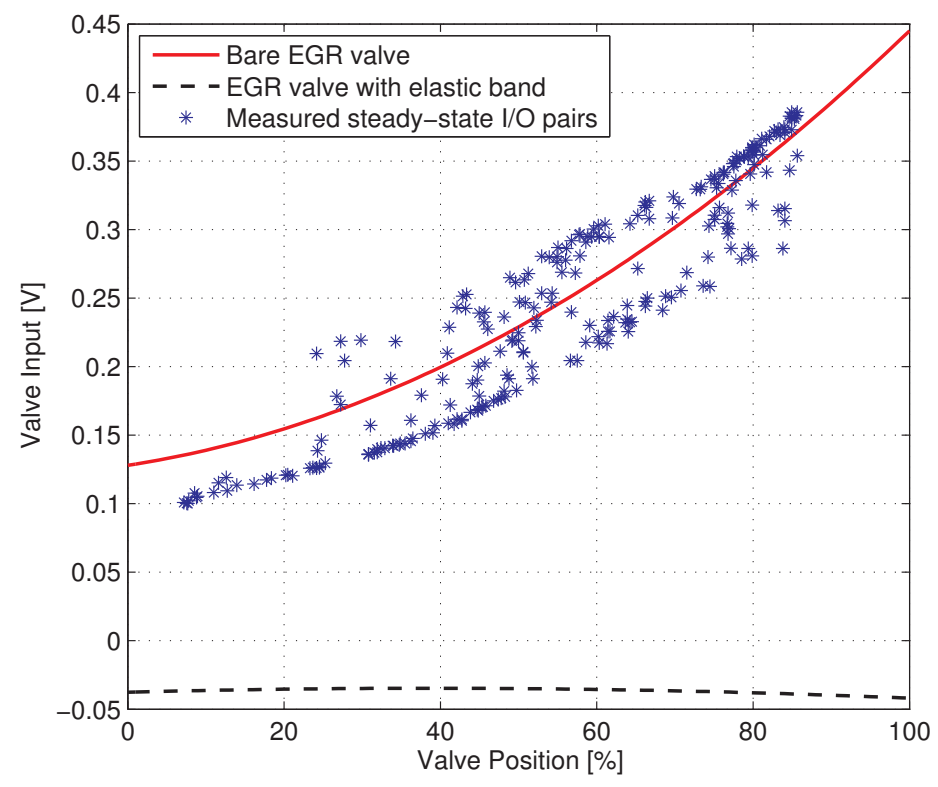

Figure 8.6: Steady-state input-output pairs and the identified function.

mated function, given by $u_{\mathrm{ff}}(r)=\left(\alpha^{*}\right)^{\top}\left[\begin{array}{lll}1 & \frac{r}{100} & \frac{r^{2}}{5000}\end{array}\right]$ using the asymptotic value $\alpha^{*}=(0.128,0.087,0.115)$ of $\alpha$ reached after the sequence of step changes of $r$ reported in Figure 8.7. In particular, Figure 8.7 shows the experiment performed by initializing $\alpha$ to zero and running the FORE control scheme (7.14), (7.2), (7.22) under a random sequence of steps for the position reference. For the experiment in Figure 8.7 . we have intentionally selected the non-convenient value $\lambda=0.04$ (corresponding to the slow blue dashed-dotted response of the lower plot of Figure 8.4 so that it is possible to appreciate the convergence of the parameters $\alpha$ to the optimal values $\alpha^{*}$ discussed in Section 7.4.3. The parameters of the adaptation inhibition mechanism in 7.22 have been tuned to $\lambda_{h}=100$ and $\bar{r}_{f}=50$ in such a way to avoid undesired transients in the trace of $u_{\mathrm{ff}}$ upon sudden changes of the reference $r$ (this was experienced in the responses of Figure 8.4 and discussed at the end of Section 8.4). Note also that as the parameters $\alpha$ get closer 

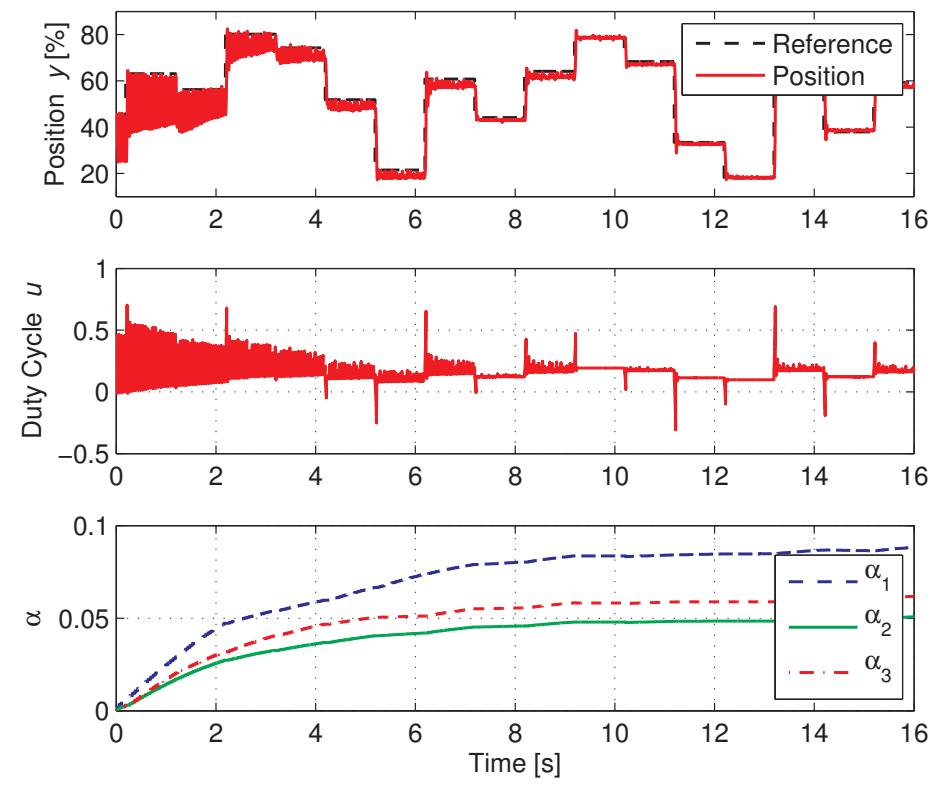

Figure 8.7: Convergence of the parameters $\alpha$ across a sequence of steps of $r$.

to their asymptotic values $\alpha^{*}$ (lower plot of Figure 8.7), the step responses in the upper plot of the figure become increasingly desirable. In particular, the typical step response experienced after the parameters have converged corresponds to the black solid response in Figure 8.9 which shows a significantly decreased overshoot as compared to the red solid step response in Figure 8.4 of the previous section (that response is also reported in Figure 8.9 using a red dashed-dotted curve). A last comment should be made about a similar experiment to that of Figure 8.7. carried out after placing an elastic band that pulled the valve open, as shown in Figure 8.1. In this case the parameters converged to the values $\alpha^{*}=(-0.0375,0.0148,-0.0096)$ and, after convergence, the step responses were very similar to the previous case. The black dashed curve in Figure 8.6 shows the estimated function $u_{\mathrm{ff}}^{*}(r)$ with this configuration using the elastic band. 


\subsection{Tracking a time-varying position reference}

Our FORE-based reset controller design is carried out addressing a set-point regulation problem, namely a piecewise constant reference position. However, in a typical application on the engine it is important to also consider the behavior of the FORE when facing a time-varying reference. In this case, when the reference variation is fast enough, the adaptation of the feedforward action is stopped by the inhibition logic 7.22 and the feedforward action, based on the previous adaptation, will be combined with the stabilizing feedback performed by the FORE. Conversely, if the reference varies slow enough, the adaptation will be active and will exploit the corresponding information also in practical cases where the reference is close to being constant but perhaps affected by noise.
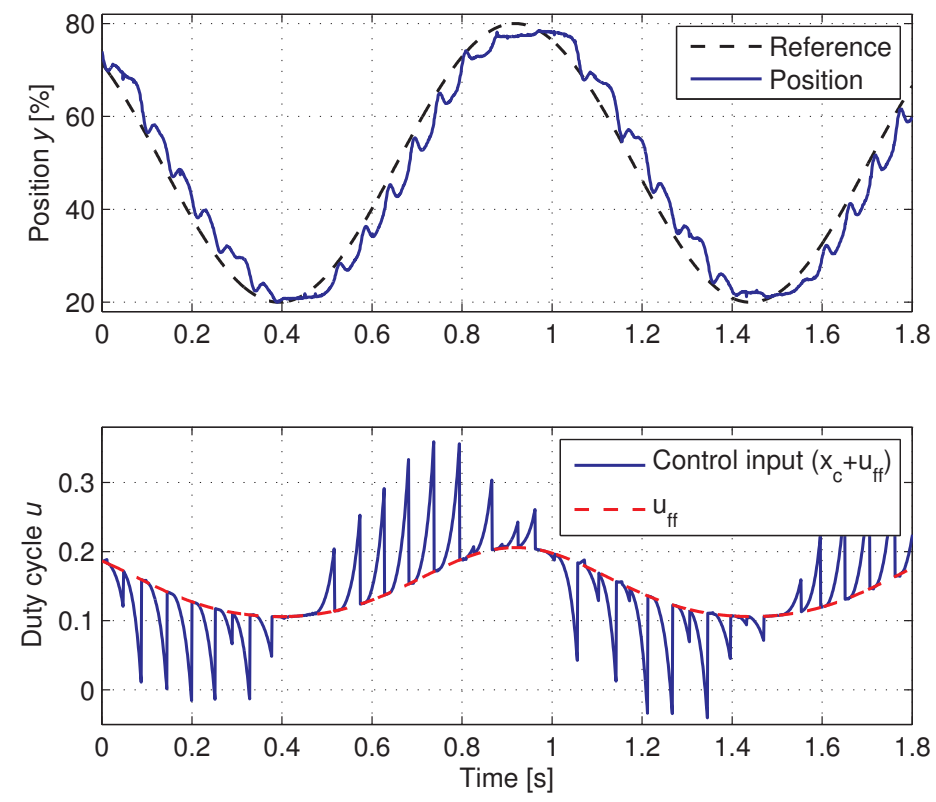

Figure 8.8: Response of the FORE control system to a time-varying reference.

An example of this behavior is shown in Figure 8.8 where the reference is a $1 \mathrm{~Hz}$ sine wave. The distorted sine wave visible as a dashed 
curve in the lower plot corresponds to $u_{\mathrm{ff}}$ and is the image of the reference through the quadratic curve of Figure 8.6. Figure 8.8 also reveals high frequency oscillations whose period depends on the parameters of the FORE. More precisely, if the FORE is fast enough, the frequency of the oscillations will be higher and the amplitude will be smaller. These oscillations represent the typical behavior of the FORE in this scenario. They are caused by the fact that the controller acts with exponential branches of the control input, that could be seen as pulses occurring when the error is large enough (if the FORE is aggressive enough). Figure 8.8 shows that once the feedforward term is adapted, the FORE control scheme achieves a reasonable tracking of the reference but an undesired ripple is to be expected due to the very nature of FORE stabilization. The size of the ripple is expected to decrease as the reference becomes slower, nevertheless for a full elimination of the ripple and perfect reference tracking, more sophisticated solutions are required, such as the one presented in Section 7.5.

\subsection{Comparison to time-optimal and PI solutions}

To provide a more complete assessment of the developed reset control scheme for the EGR valve, we develop in this section two alternative solutions for a step reference change from $20 \%$ to $60 \%$ of the valve position. In particular, we compare the step responses obtained using the FORE control schemes of Sections 8.4 and 8.5 to the responses obtained using

1. a time-optimal open-loop control strategy, leading to the requested position change in minimum time; the arising response (blue dashed curve of Figure 8.9 can be thought of as the best possible achievable performance using a robust feedback controller;

2. a standard PI controller; the arising response (green solid curve in Figure 8.9 can be understood as the typical industrially adopted solution. 

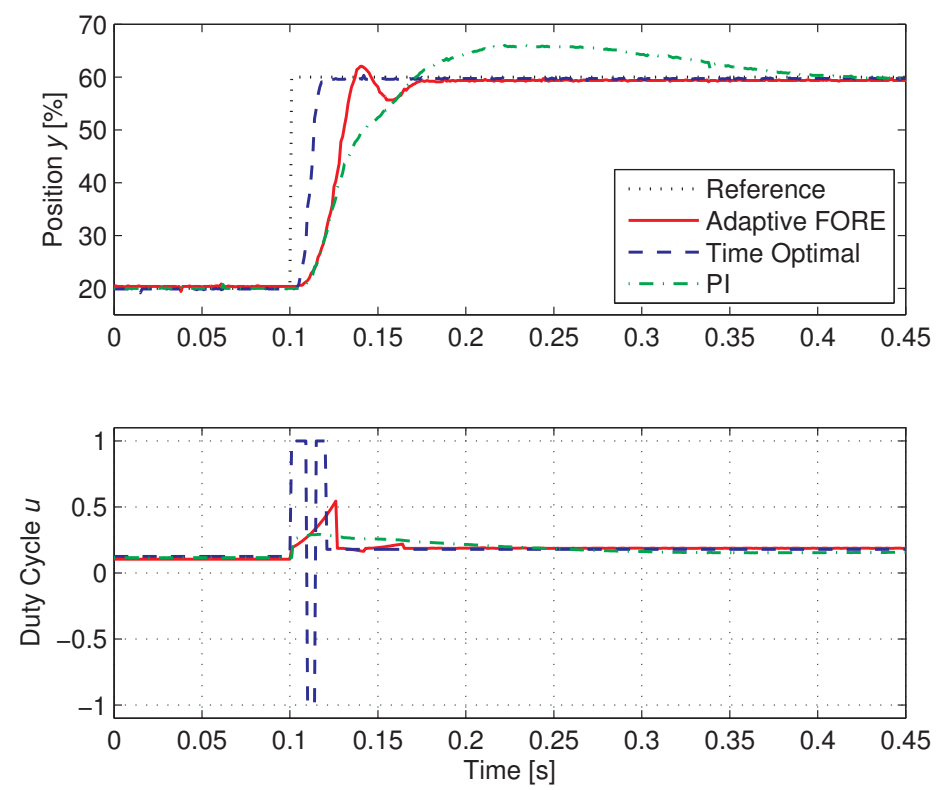

Figure 8.9: Comparison among the FORE, PI and time-optimal step responses.

More specifically, the time-optimal open-loop control sequence (blue dashed curve in the lower plot of Figure 8.9 has been determined from the knowledge that the optimal input is a bang-bang signal which remains at the maximum allowable input values until the new steadystate is reached. In particular, by classical time-optimal control results (see, e.g., Pontryagin et al., 1962, Thm 10, page 120]) for a third order plant, three time intervals where the input switches between the upper and lower saturation levels are required 1 . The three time intervals characterizing the time-optimal input sequence were empirically found to be $T_{1}=8.95 \mathrm{~ms}, T_{2}=5.58 \mathrm{~ms}, T_{3}=5.2 \mathrm{~ms}$, while the steady-state inputs before and after the bang-bang burst were given by the black dashed curve of Figure 8.6 evaluated at $20 \%$ and $60 \%$. Note that the time-optimal solution is only useful for comparison purposes and cannot be used on the real plant because it is a feed-forward signal extremely

\footnotetext{
${ }^{1}$ It should be emphasized that a third order model, also including the electrical time constant of the valve's electrical motor, is necessary to suitably characterize the response in the time scale characterized by the time-optimal response.
} 
sensitive to parameter variations. Indeed, the dashed curve of Figure 8.9 was the best one among a series of experiments where often the output exhibited over or undershoots due to the uncertainties affecting the valve response. Moreover, in a situation with strong disturbances, such as the Diesel engine implementation of the next section, it is essentially impossible to compute the time-optimal sequence of inputs for each requested valve positions.

Regarding the tuning of the PI gains, the following approach was taken. A first set of gains was determined by means of the Simulink Control Design PID Tuner, a tool available within the Matlab environment. Among other things, this tool allows to specify the desired closed-loop response time and, based on a given plant model, it computes the PI parameters inducing that response time with an adequate robustness level. Using the identified model (8.1), the tool was used with decreasing values of the requested response time, until the simulation response was sufficiently representative of the experimental one. Finally, the corresponding PI gain selection was slightly adjusted for improving the performance on the step reference change from $20 \%$ to $60 \%$. This led to the final selection $\left(K_{p}, K_{i}\right)=(0.004,0.1)$. Note that industrial PI solutions often use nonlinear gains to increase performance and robustness so this comparison is not comprehensively illustrative. However, the comparison carried out on the Diesel engine, reported in Section 8.9, reveals that the qualitative results anticipated by the curves of Figure 8.9 are confirmed by the tremendous performance increase (the mean squared PI position error is more than four times larger than the mean squared FORE position error) shown by the experiments.

Inspecting the four responses of Figure 8.9 reveals that the performance of the adaptive FORE with parametrized feedforward (black solid) is quite close to the time optimal response. Indeed, the rise time is roughly doubled and the settling time is approximately $70 \mathrm{~ms}$ as compared to the $20 \mathrm{~ms}$ achieved by the time-optimal response. The performance obtained using the PI controller (green solid) is not even comparable to the extremely aggressive performance of the proposed FORE scheme. Finally, as illustrated at the end of the previous sec- 
tion, the comparison between the red dashed-dotted and the black solid curves illustrates the advantage of the scheme illustrated in Section 8.5 as compared to the one of Section 8.4. especially in terms of overshoot reduction.

\subsection{Implementation on the Diesel engine testbench}

We discuss here the FORE controller implementation on a 2 liter 4 cylinder passenger car turbocharged Diesel engine, which is designed to meet the EU5 emission legislation. The engine features a common rail injection system, a variable geometry turbine turbocharger with charge air cooling and cooled high pressure exhaust gas recirculation. The engine is equipped with production standard sensors and it was operated on a highly dynamical engine test bed at the Johannes Kepler University (JKU) in Linz. The development engine control unit (ECU) was connected to a real-time hardware-in-the-loop system for data acquisition and control. All the experiments were carried out by the use of a dSpace rapid prototyping system to control the EGR valve positioning, whereas the remaining functions of the engine control unit were kept at production standard.

For this engine, we implemented the hybrid EGR valve position controller described in Section 8.5 with the optimized value $\lambda=0.32$, as discussed in Section 8.4 and using the filter in Section 8.3 with $\epsilon=0.005$. Note that the selected value of $\epsilon$ is larger than the one used for the laboratory experiments $(\epsilon=0.001)$, because the noise level is higher in the Diesel engine test bed signals.

To suitably implement our controller, the bypass feature available for certain ECU signals has been used. This allowed us to leave unchanged the higher level controller, while the production standard EGR valve position controller coded in the ECU was replaced by the FORE controller, which was running in the dSpace hardware-software setup connected to the testbench. Moreover, the testbench allowed for the monitoring of several engine outputs whose behavior is reported in this section.

Notice that since the valve recycles exhaust gasses, it is subject to 


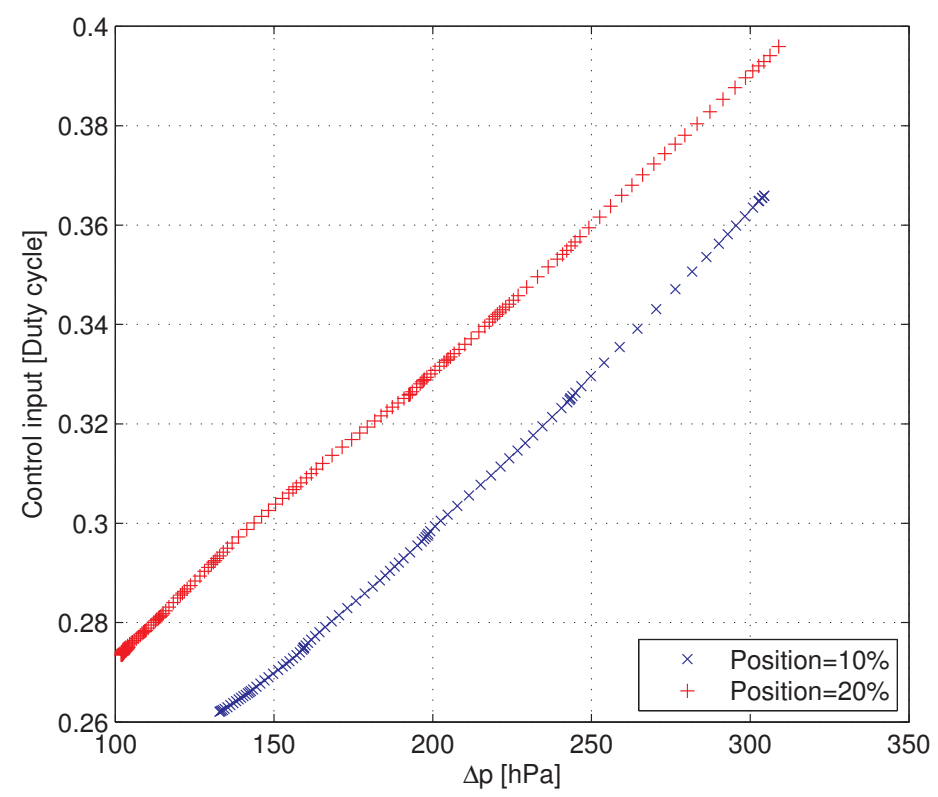

Figure 8.10: Plot of the $\left(u_{\mathrm{ff}}, \Delta p\right)$ pairs given with a fixed position reference for the EGR valve.

aging, in the sense that soot particles may interfere with the valve operation, e.g., increasing the valve stiction when it is fully closed.

When the engine is running, the main disturbance is caused by the flux of the exhaust gas which pushes the EGR valve in the closing direction. A peculiarity of this disturbance is that closing the valve causes an increased pressure difference, therefore an increased disturbance. This effect may induce closed-loop oscillations if the controller is not aggressive enough. Moreover, the disturbance may oscillate at the steady state due to the oscillations of the pressure drop across the valve. For illustration purposes, Figure 8.10 shows the steady-state feedforward input as a function of the pressure difference $\Delta p=p_{i}-p_{e}$ between the intake manifold pressure $p_{i}$ and the exhaust manifold pressure $p_{e}$. Each curve corresponds to the steady-state feedforward value when the valve is regulated at a constant position. Experimentally, the curves were obtained by running the engine testbench with a constant EGR position reference and changing the engine operating 
conditions (therefore $\Delta p$ ) while recording several steady-state input values. Each recorded value is represented by a "+" or an " $x$ " in Figure 8.10. Since the dependence on $\Delta p$ is essentially affine, we use the FORE controller with the same parameters used in Section 8.5 and with $\Psi(r, \Delta p)=\left[2.5 r / 100 r^{2} / 5000 \Delta p / 500\right]$, so that $\alpha \in \mathbb{R}^{4}$. With this selection, the feedforward action 7.20 comprises a linear term in $\Delta p$ to capture the behavior of Figure 8.10 in addition to the remaining terms used in the laboratory experiments. The selection above for $\Psi$ ensures that the entries of $\Psi$ all have the same order of magnitude during average operations, except for a larger value of the first bias term (namely the constant term of the polynomial function $\left.u_{\mathrm{ff}}=\Psi(r, \Delta p)^{\top} \alpha\right)$. This ensures that when the engine operates close to zero valve position, where our approximation is inaccurate, the states $\alpha_{2}, \alpha_{3}, \alpha_{4}$ do not deviate too much from the values providing good performance in midrange operating conditions.

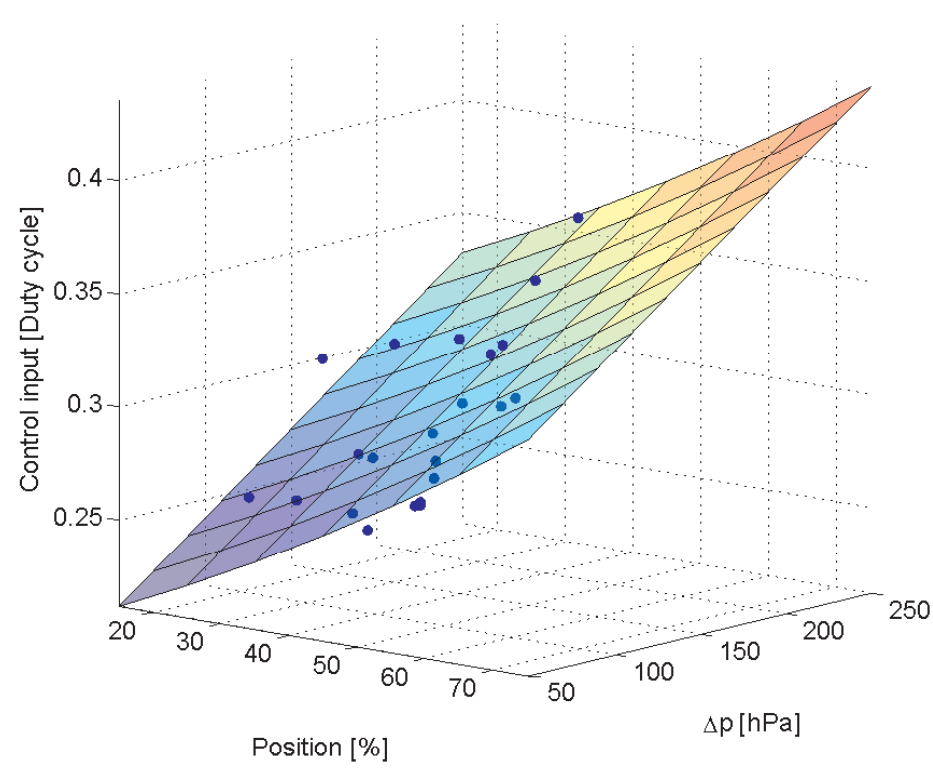

Figure 8.11: Steady-state map $\Psi(r, \Delta p)^{\top} \alpha$ after the experimental adaptation. The blue dots correspond to the measured steady-state input/output values during the experiment. 
Figure 8.11 shows the feedforward surface $\Psi(r, \Delta p)^{\top} \alpha$ after an experimental adaptation of $\alpha$ with changing engine conditions. The blue dots represent the steady-state input-output pairs observed during the experimental run. This function essentially extends the curve of Figure 8.6 to the case where also $\Delta p$ is considered in the feedforward parametrization.

Remark 8.1. Since in the Diesel engine application the FORE control system is subject to temporary disturbances mostly arising from the closed-loop effects of the VGT, practical experience revealed that it is advantageous to saturate the update term $\frac{\Psi}{\Psi^{\top} \Psi} x_{c}$ in $7.22 \mathrm{~b}$ so that excessively large updates of $\alpha$ due to transient disturbances do not occur. For our application we used $\frac{\Psi}{\Psi^{\top} \Psi} \sigma_{0.2}\left(x_{c}\right)$, where $\sigma_{M}(s)=$ $\operatorname{sign}(s) \min \{|s|, M\}$ is the standard scalar saturation function. Similarly, it is possible to saturate the right hand side of the $x_{c}$ state equation in 7.2 or even to limit the maximum value of $x_{c}$ in such a way that transient disturbances do not cause excessively large deviations of the plant input from the steady-state. For our application we limited $x_{c}$ in the set $[-0.3,0.3]$. Note that this does not correspond to limiting $u$ in that set due to the presence of $u_{\mathrm{ff}}$ in the second equation of 7.14. An alternative to this is to design a more conservative controller by reducing the FORE parameters. However, using saturation gives the advantage of not affecting the aggressive small signal behavior and only toning down the large signal responses.

\subsection{Experimental results}

A first set of experiments carried out to assess the FORE controller performance was performed bypassing the EGR position reference coming from the higher level controller and requiring a step change of the EGR valve position during a steady operation phase of the engine. Therefore, parallel experiments to the ones of Figure 8.9 are obtained. In particular, Figure 8.12 was obtained with the engine running at speed $2200 \mathrm{rpm}(\approx 37 \mathrm{~Hz})$ and fuel amount of $30 \mathrm{mg} /$ cycle. Here, the solid curve corresponds to the response of the FORE controller and should be compared to the dashed curve, obtained when delivering the step 
reference to the ECU production standard controller, which is based on a gain scheduled PI plus feedforward architecture. The high frequency oscillations on the measured position (upper plot) are caused by the air flow peaks through the EGR valve caused by the combustion cycles. Their frequency (roughly $75 \mathrm{~Hz}$ ) is four times larger than a complete cycle of the engine (roughly $18 \mathrm{~Hz}$-note that each cycle requires two engine revolutions therefore this frequency is half of the engine rotation frequency). The third trace reveals also oscillations of $\Delta p$ which exhibit the two above discussed fundamental frequencies of 18 and $75 \mathrm{~Hz}$. The feedforward action $u_{\mathrm{ff}}=\Psi(r, \Delta p)^{\top} \alpha$ is effective at making this disturbance almost invisible on the position error, where only the residual $75 \mathrm{~Hz}$ component is visible. The rest of the job is carried out by the exponentially diverging branches of the input $u$. The lower plot of Figure 8.12 shows the adapted parameters $\alpha$. Notice that the parameters remain essentially constant because they have already converged to the steady-state values, nevertheless, in accordance with the discussion of Section 8.8 , the bias term $\alpha_{1}$ exhibits more visible variations due to the choice of the normalization factors in the function $\Psi(r, \Delta p)$.

A second, perhaps more significant, test carried out on the engine testbench was to compare the EGR valve positioning accuracy when using the ECU production standard controller and the proposed FORE controller through an urban part of the New European Driving Cycle (NEDC), which lasts roughly 198 seconds. As customary, this test cycle corresponds to providing suitable torque and speed references to the engine, based on a conventional behavior of a virtual driver on a prescribed road. This test simulates a typical situation in an urban environment2,

The response of the FORE is compared to the one of the production standard ECU controller in Figures 8.13 and 8.14. Such a comparison clearly infers that the FORE allows for a much faster tracking of the reference position. In particular Figure 8.13 and the two top plots of Figure 8.14 clearly reveal the higher precision of the hybrid controller. Looking at the lower plot of Figure 8.14 it is also possible

\footnotetext{
${ }^{2}$ The extra-urban part of the NEDC was not considered as it is less demanding from the point of view of the presented EGR control system.
} 

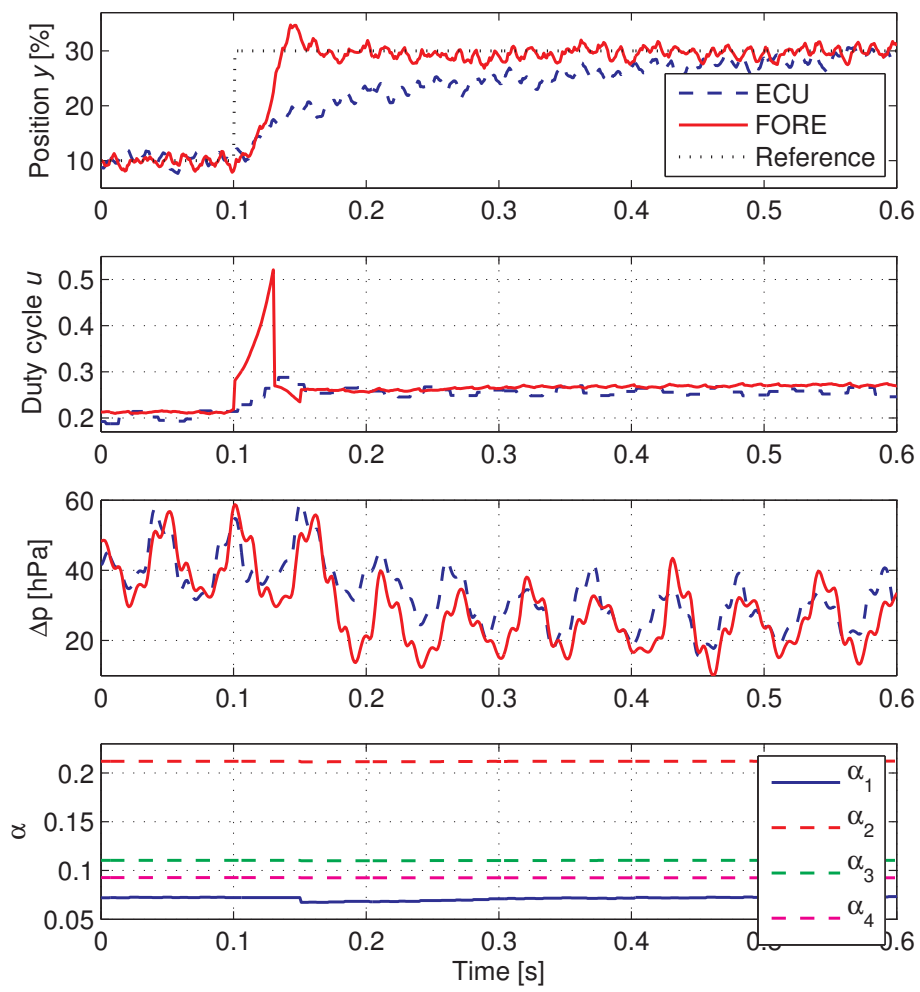

Figure 8.12: Comparison of the step responses of the proposed FORE controller and of the ECU production standard controller.

to appreciate the impact on the $N O_{x}$ emissions when using the FORE scheme. In particular, not surprisingly, the most relevant difference of the $N O_{x}$ emissions is visible whenever an opening of the EGR valve is requested by the higher-level control system (see the transient response around time $t=117)$. Indeed, the reduced position tracking error given by the FORE controller ensures a prompt gas recirculation and a reduced amount of emissions. One may also expect that the large position error experienced by the ECU control system in the time interval $t \in[119,120]$ could have an impact on the emissions but this is not the case because this error does not impact much the EGR flow. Instead in the previously commented phase, where the EGR valve 

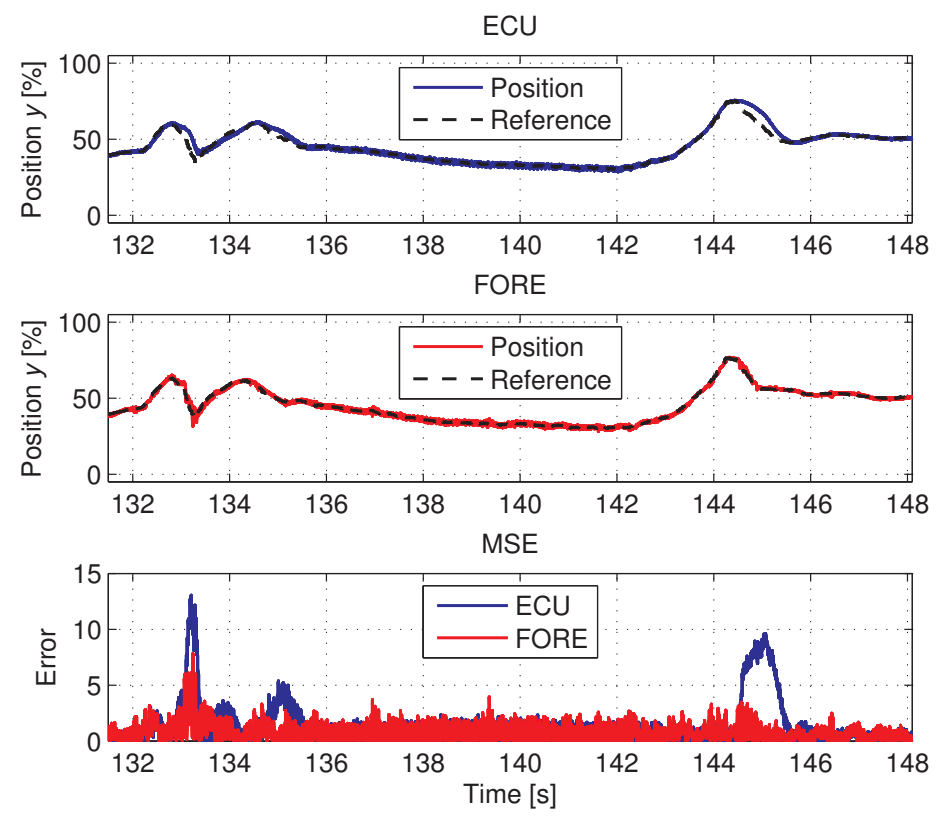

Figure 8.13: ECU and FORE responses on a portion of the NEDC.
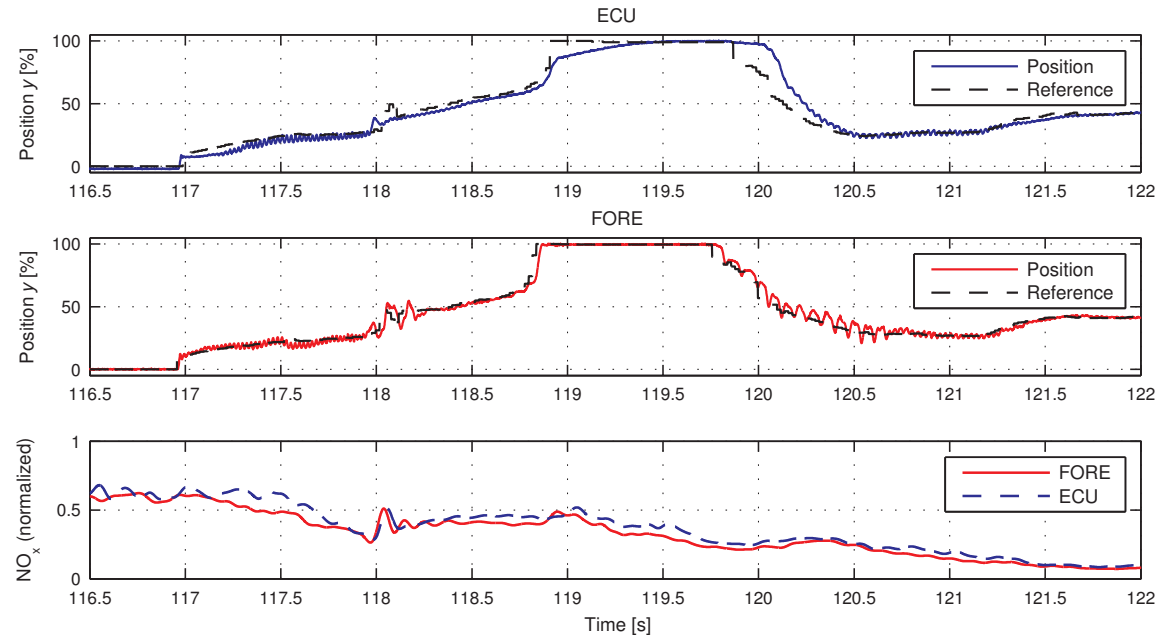

Figure 8.14: ECU and FORE responses on a portion of the NEDC together with $N O_{x}$ emissions. 


\begin{tabular}{|c|c|c|}
\hline Controller & MSE & Percentage \\
\hline \hline ECU & 6.6794 & $100 \%$ \\
\hline FORE & 1.5343 & $23 \%$ \\
\hline
\end{tabular}

Table 8.1: Mean squared position error of the FORE and ECU control systems along the NEDC cycle.

is almost closed, the FORE position controller accuracy has a strong impact on the EGR flow.

To numerically quantify the gap between the two controllers, we also consider the mean squared position error achieved by the two controllers in the considered NEDC cycle, namely

$$
M S E=\frac{1}{|\mathcal{I}|} \sum_{i \in \mathcal{I}} e_{i}^{2}, \quad \mathcal{I}=\left\{i \in\{1, \ldots, n\}: r_{i} \neq 0\right\}
$$

where $e_{i}$ corresponds to the $i$-the sample of the difference between the position reference (dashed line in Figures 8.13 and 8.14 ) and the valve position (solid lines in the same figures). The sampling period of the signals is $1 \mathrm{~ms}$ so that during the whole $198 \mathrm{~s}$ NEDC urban cycle there are $n=198000$ samples. Note, however, that we evaluate the MSE averaging only the values of the error where the reference position of the valve is not zero. This is due to the fact commented above that with zero reference the ECU control system switches to the "fast closure" operating mode where the position measurement signal is negative (see the discussion above and the corresponding time intervals in Figure 8.14 and this would lead to (incorrectly) increased values of the MSE for the ECU control system.

The values obtained using the two schemes are listed in Table 8.1 where it appears that the FORE control system reduces to less than one fourth the MSE value. This is not surprising if one takes into consideration the curves of Figures 8.13 and 8.14 which reveal a substantial improvement of the valve positioning performance. 


\subsection{Overview}

Power-split transmission systems for traction drives comprise different branches where power can be directed and manipulated in order to achieve suitable efficiency goals (see Mueller et al. [2014] and references therein). In recent years, high-technology solutions have been developed with the goal of reducing the energy consumption in offhighway mobile machines. One such example is the Hydromechanical Variable Transmission (HVT) developed at Dana Rexroth Transmission Systems (DRTS). This device combines the mechanical and hydrostatic travel drive to join the best features of both technologies. In particular, with such device the energy consumption of the vehicle is reduced and the life cycle costs are also decreased due to the high level of automation within the hydro-mechanical components. The HVT developed by DRTS includes a hydrostatic variator and a mechanical power branch whose interplay is modulated by way of variable hydrostatic pump and motor, and by the action of proportional controlled wet disc clutches allowing the automatic control system to switch among several drive ranges (see Mueller et al. 2014 for details). These clutches are then suitably actuated by electro-proportional valves modulating 
the oil pressure. One key feature of the overall control system is an accurate control system for the current flowing within the electrovalves, which has a direct impact on the accuracy and precision of the clutches operation.

According to the detailed description in Mueller et al. 2014, the HVT transmission developed at DRTS, schematically represented in Figure 9.1 as integral part of a Wheel Loader (WHL) drive train, includes among its many features the action of two clutches whose role is to suitably adjust the range of the two branches, so that desirable operating conditions are met by the device for increased performance and comfort.

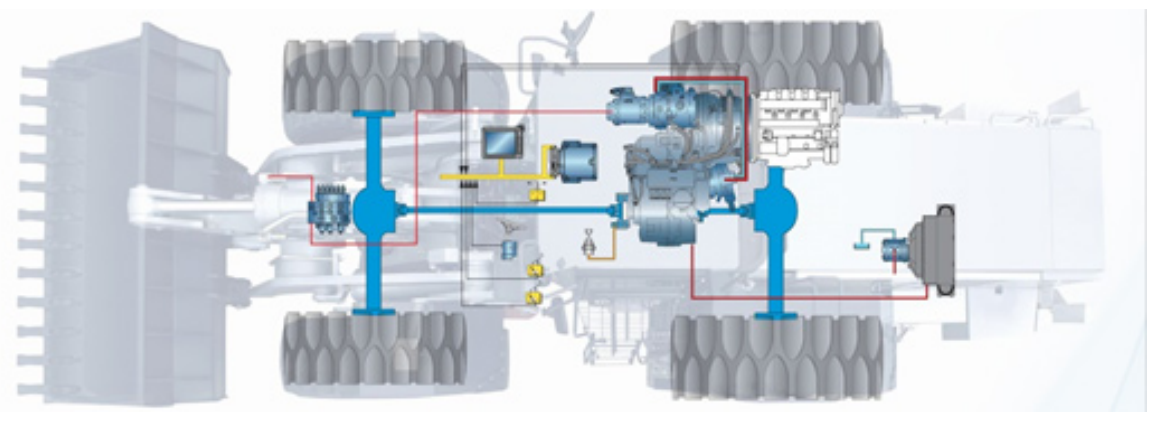

Figure 9.1: Scheme of the power-split transmission developed by DRTS.

To the end of suitably actuating the clutches, it is fundamental that the oil pressure before the clutches be accurately regulated in such a way that the clutch discs reach with a precise filling operation, the so-called kiss point, before engaging. Then, no dead time (or time lag) is experienced by the higher level control system when demanding the engagement of the clutch. This critical goal can be accomplished by accurately modulating the current flowing in the electrovalves regulating the fluid pressure of the clutch piston acting on the clutch discs. In its current HVT architecture, DRTS uses proportional electrovalves whose operation stands between the cheap and inaccurate "switching valve" solution and the fragile "servo-valve" solution. Proportional hydraulic valves became commercially available in the mid 1980s and became viable alternatives to the more expensive and fragile servo-valves. They 
allow for infinite positioning of the spool, thus providing infinitely adjustable flow volumes. These valves are electrically operated by proportional built-in solenoids whose goal is to provide a smooth and continuous variation in flow or pressure by suitably manipulating the current flowing in the solenoid. What permits the opening of the ports is the movement of the spool that is regulated by the balance of the forces acting on it. In particular, the electrical input passing through the coils of the solenoids produces an electromagnetic force that is opposed by the force of the spring and this infinitesimal balancing of forces permits the opening/closing of the valve. The difference with the conventional directional valves, as said above, is that the opening/closing can be modulated to vary the flow and pressure. Summarizing this means that an opening of the valve (movement of the valve spool) is proportional to the magnitude of the milliamp current applied to the valve. As a consequence, besides the nonlinear relationship between electrical current and fluid pressure, which should be suitably inverted (possibly using adaptive schemes), the pressure and flow can be regulated by these valves by only focusing on the electrical dynamics, which is linear and can be written as a standard RL model:

$$
\dot{I}=-\frac{R}{L} I+\frac{1}{L} V+\bar{d},
$$

where $I$ is the current flowing in the solenoid, $V$ is the applied voltage, $\bar{d}$ is a constant input bias and $R$ and $L$ are the parameters of the corresponding RL passive circuit.

Accurate tracking of the time-varying reference for the current flowing in the valves of the power-split transmission system of DRTS allows to obtain extreme performance in the overall device. This section then reports on the experimental results obtained when using the control scheme presented in Section 7.5 for obtaining perfect tracking of a time varying current reference profile, which may be then adjusted in order to achieve suitable pressure/flow regulation in the clutch. The advantage of the adopted scheme is that it is able to adapt to slow variations of the parameters $R, L$ and $d$ in equation (9.1) and that is robust enough to unmodeled phenomena, such as the sampled-data implementation of the control scheme and the PWM nature of the power amplifier operating the valve (see the next section for details). 


\subsection{Application to the valve model and simulation results}

When focusing on the experimental system described in Section 9.1, the adaptive scheme of Section 7.5 can be applied by identifying the linear dynamics in (7.16) with the electrical equations of the valve, reported in 9.1$)$.

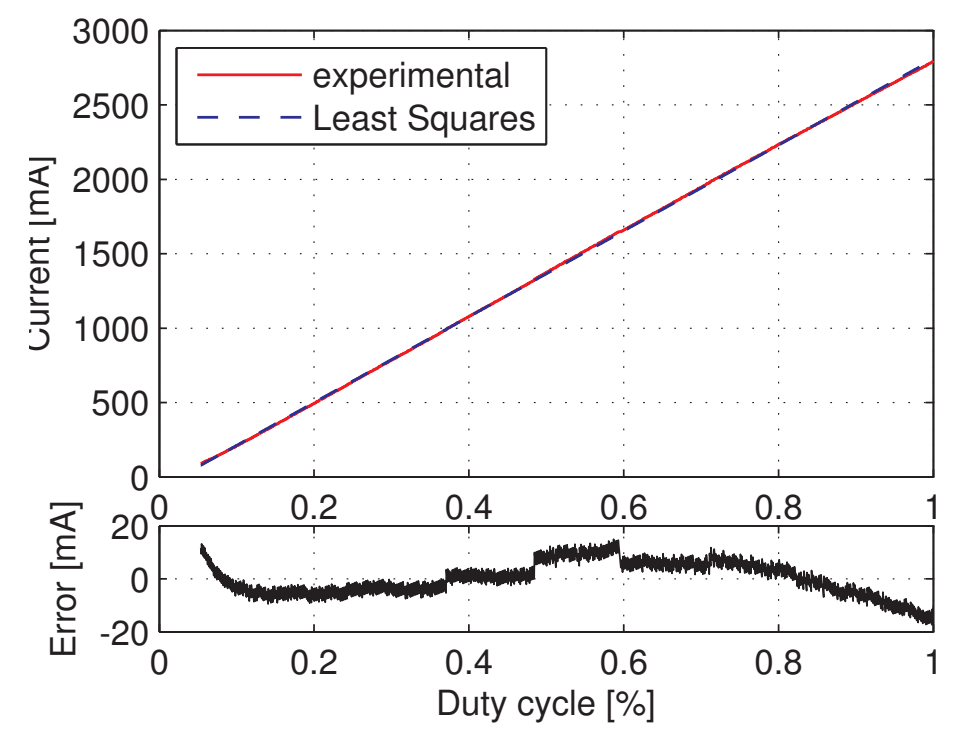

Figure 9.2: Linear fitting of the static experimental measurements.

Some preliminary experiments have been carried out under quasistatic behavior of the valve, in order to validate the correctness of the parametrization in (7.23). In particular, a number of increasing step voltages have been applied to the valve, and the corresponding output current has been sampled and averaged after it reached its steady state value upon each value of the piecewise constant input. The corresponding experimental results are reported by the red solid curve in the upper plot of Figure 9.2. From this curve it is evident that the proposed parametrization well suites this application, at least under static conditions (that is, when $\dot{r}=0$ ). The blue dashed line in the upper plot of Figure 9.2 shows the linear least squares fit of the experimen- 
tal curve, and the lower plot in the figure highlights the arising error, that can be considered acceptable for the specific application at hand. Note also that the parameters associated to this curve vary with time, mostly depending on the temperature of the valve, but also depending on aging and other unpredictable effects on the electro-mechanical valve.

\begin{tabular}{|c|c|c|}
\hline Component & Value & Units \\
\hline$R$ & 10 & $\Omega$ \\
\hline$L$ & 70 & $m H$ \\
\hline $\bar{d}$ & -3 & $\mathrm{~A} / \mathrm{s}$ \\
\hline
\end{tabular}

Table 9.1: Parameters used in the simulations of Section 9.2

For simulation purposes, we have selected the parameters reported in Table 9.1 which are compatible with the experimental curves shown in Figure 9.2. Regarding the parameters of the FORE, according to suitable experimental tests reported in Cordioli [2014], they have been selected as $a_{c}=50, b_{c}=15$ and $\lambda=0.4$ in both simulations and experiments. The dwell time parameter in $(7.2)$ has been selected as $\rho=0.01$. It has been noted that this parameter does not really affect the type of responses experienced from the closed loop.

Simulations have been carried out for the feedback between plant 7.16) with $a_{p}=-R / L$ and $b_{p}=1 / L$ (see (9.1) ) and controller 7.2), 7.14, 7.29, (7.28), 7.27) introduced in the previous section. In all of them, the function $\varphi$ in 7.28 has been selected based on the nominal values of the parameters in Table 9.1. namely $\varphi(\tau)=$ $0.022997 \exp (48.882 \tau)+0.007931 \exp (-141.74 \tau)-0.030928$. However, these values have been perturbed in the plant by simulating the controller over a plant with $\hat{R}=1.2 R$ and $\hat{L}=0.8 L$ (note that we have perturbed $R$ and $L$ in opposite directions to ensure that the time constant $L / R$ was suitably perturbed too). An input matched disturbance of $-3 V$ has been introduced in the simulations that, according to dynamics 7.16 results in a selection of $\bar{d}=-3 / b_{p}$. In all our simulations, a band limited white noise with power $10^{-8}$ and sampling time of $1 \mathrm{~ms}$ 
has been added to the current sensing feedback signal.
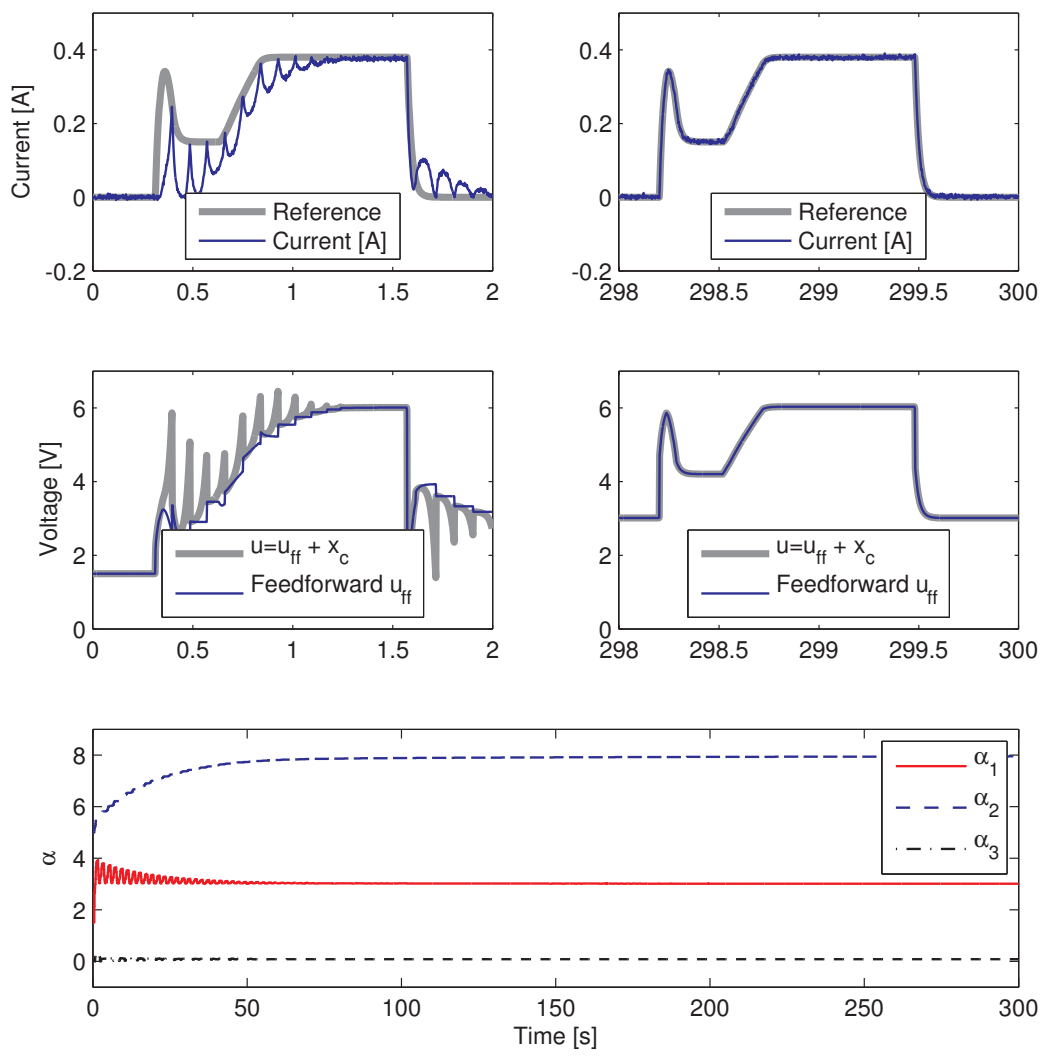

Figure 9.3: Simulation results with the Toolbox Sanfelice et al. 2013. Two upper left plots: output currents (top) and input voltages (bottom) at the beginning of the adaptation. Two upper right plots: output currents (top) and input voltages (bottom) at the end of the adaptation. Down plot: time-evolution of the parameters $\alpha_{1}, \alpha_{2}$ and $\alpha_{3}$.

Two different simulations are shown in Figures 9.3 and 9.4 . In the first case of Figure 9.3 we have implemented the FORE scheme on a simulation file involving a continuous-time implementation based on the hybrid simulation tool of Sanfelice et al. 2013. In the second case the controller has been discretized with the sampling time of $2 \mathrm{~ms}$ and the hybrid logic has been encoded in a StateFlow diagram which can 

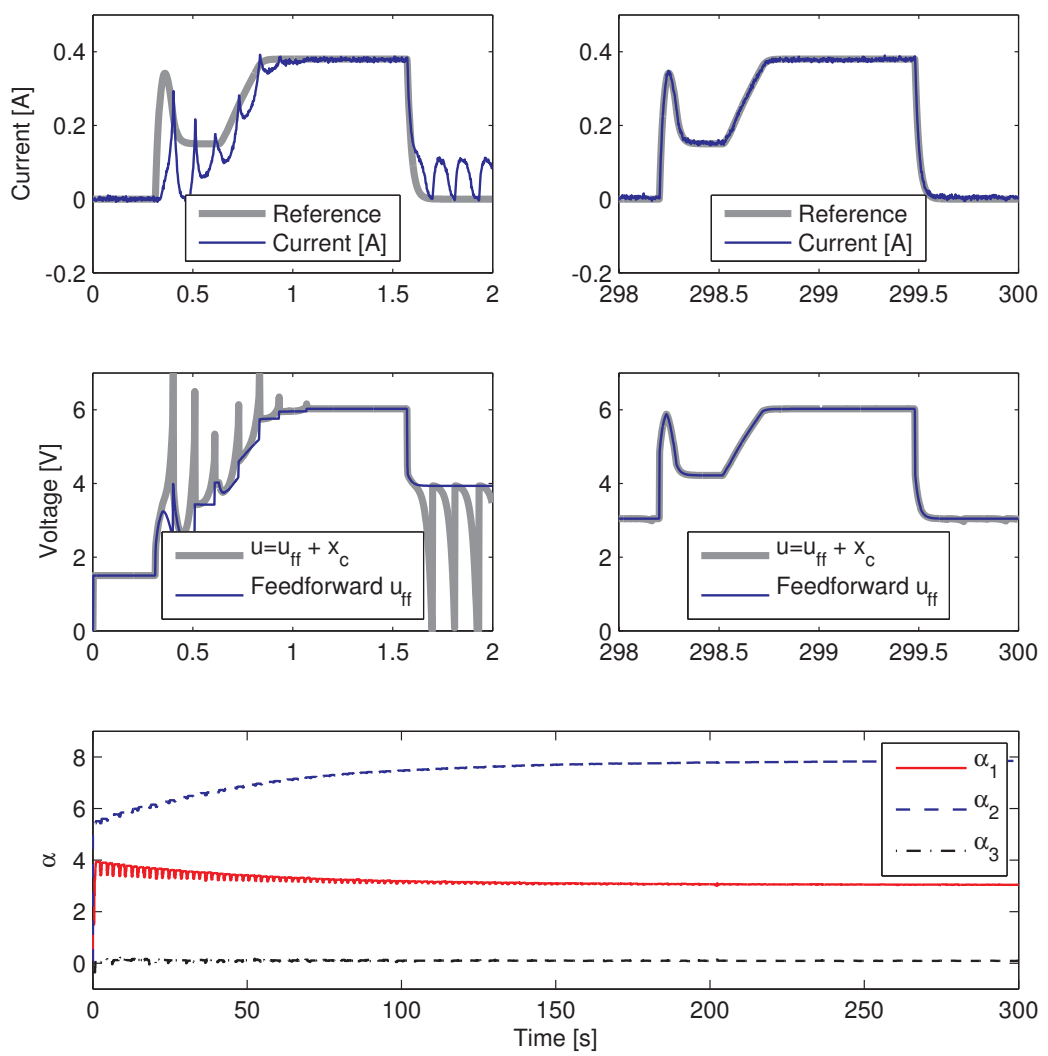

Figure 9.4: Simulation results of the sampled-data approximated controller implementation. Two upper left plots: output currents (top) and input voltages (bottom) at the beginning of the adaptation. Two upper right plots: output currents (top) and input voltages (bottom) at the end of the adaptation. Down plot: time-evolution of the parameters $\alpha_{1}, \alpha_{2}$ and $\alpha_{3}$.

be easily converted into the $\mathrm{C}$ source code automatically generated by the TargetLink software described in the next section. Moreover, the exponentials in the function $\varphi$ have been implemented approximatively using a second order Taylor expansion to allow for real-time implementation in the available hardware.

The simulations represent a 300 seconds time interval where the valve current reference profile corresponding to the dashed gray curve 
in the upper left plot is indefinitely repeated. The parameters $\alpha$ are initialized at half of their exact values $\alpha^{*}=\left[\begin{array}{lll}-3 \hat{R} & \hat{L}\end{array}\right]$ (see 7.25$)$ ). The two upper left plots in Figures 9.3 and 9.4 show output currents (top) and input voltages (bottom) to the valve at the beginning of the adaptation, where poor tracking is achieved and most of the control action comprises the feedback action given by $x_{c}\left(u_{\mathrm{ff}}\right.$ is very small). The right counterparts show the same signals at the end of the simulation, after the adaptation has converged to reasonable estimates and most of the control action is performed by the feedforward component $u_{\mathrm{ff}}$ (the feedback action is essentially not visible). In both cases perfect tracking is achieved. The larger plot in the lowest part of both figures shows the evolution of the parameters $\alpha$. Note the significant difference in evolution arising from the approximated sampled-data implementation of Figure 9.4 and also caused by the approximated implementation of the exponential function. Asymptotic convergence is however reached in both cases.

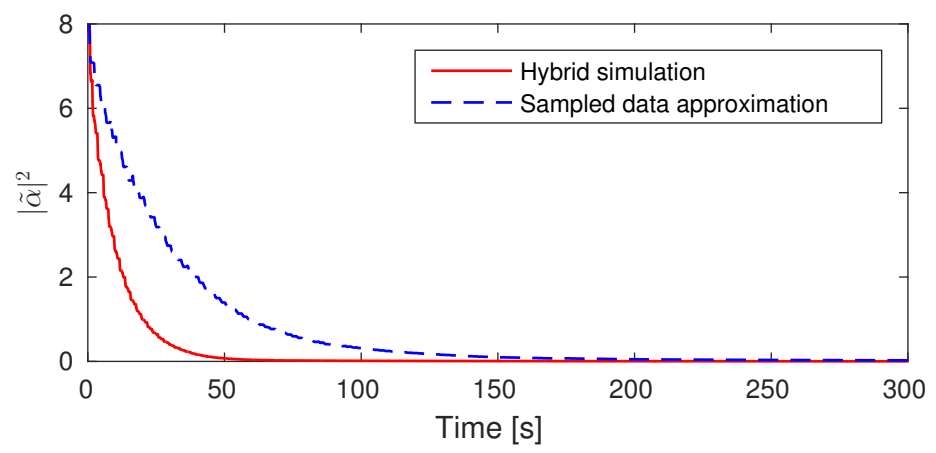

Figure 9.5: Evolution of the Lyapunov-like function $|\tilde{\alpha}|^{2}$ for the two simulations of Figures 9.3 and 9.4 .

Additional information can be extracted by looking at Figure 9.5. where the Lyapunov-like function $|\tilde{\alpha}|^{2}$ is represented for the two cases, thus clearly showing that this function decreases to zero thereby implying that parameters $\alpha$ converge to $\alpha^{*}$. 


\subsection{Experimental tests}

The simulation results of the previous section correspond to the socalled "Model-In-the-Loop" (MIL) implementation of the proposed controller, implemented using standard Simulink tools. For industrial application, the code transferred to the ECU of the power-split transmission must however be written in $\mathrm{C}$ language, compiled and flashed to the ECU. The $\mathrm{C}$ code has been automatically generated using the TargetLink software. The TargetLink generation involves associating a suitable variable (with its own precision and mainly uint8, sint8, uint16, sint16) depending on the range of variation of the related quantity (maximum value and maximum precision). For the proposed reset law, a critical variable appeared to be the controller state variable $x_{c}$ whose required precision becomes quite large once the feedforward parameters $\alpha$ are close to the actual values $\alpha^{*}$ and very small feedback corrections are necessary. In that part of the controller, 32 bit variables have been necessary to avoid freezing of the related adapted parameters.

The TargetLink software allows for automatic testing of the generated C code against the plant model (Software-In-the-Loop or SIL), which has been compared to the MIL results of the previous section. One resulting response is shown in Figure 9.6 that provides comparable results to those of Figures 9.3 and 9.4 . For the SIL simulation, unperturbed plant parameters were used so that the feedforward state $\alpha$ converges to slightly different values. Different references have been used in these tests and all gave satisfactory results, but are not reported here due to space constraints.

A few experimental tests have been performed on the physical electrovalve connected to the ECU by using TargetLink to generate the $\mathrm{C}$ code. The ECU is then connected to a hardware dSpace simulator, called Hardware-In-the-Loop (HIL), where the electrical signals reaching the ECU correspond to the ones present in the actual power-split transmission. For our test, the relevant part is that the plant connected to the ECU is not anymore a model but is the actual experimental valve. While some of the mechanical components of the power-split hardware are simulated by the HIL, the actuators and sensors are experimental, and their physical quantities are suitably monitored by the HIL to 

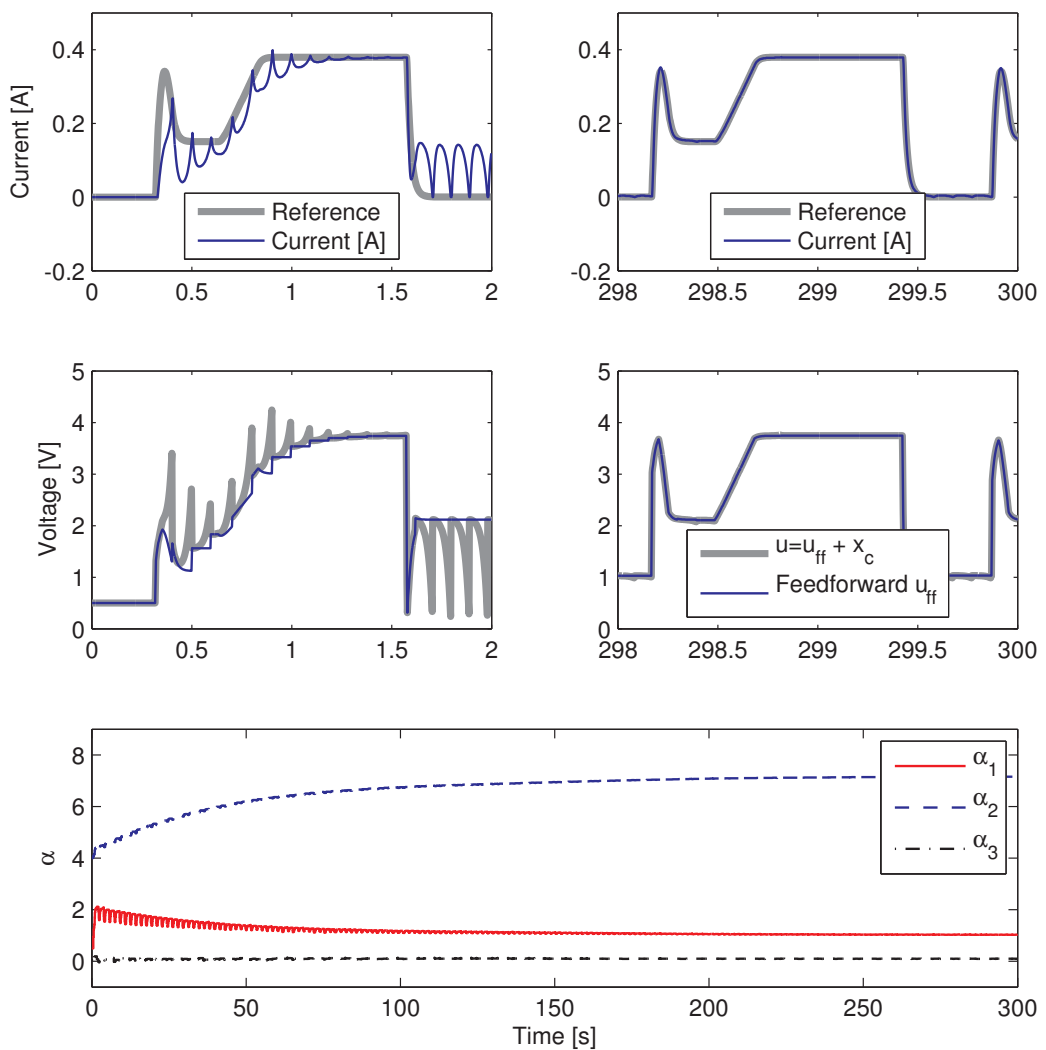

Figure 9.6: Results of the SIL source code implementation. Two upper left plots: output currents (top) and input voltages (bottom) at the beginning of the adaptation. Two upper right plots: output currents (top) and input voltages (bottom) at the end of the adaptation. Down plot: time-evolution of the parameters $\alpha_{1}, \alpha_{2}$ and $\alpha_{3}$.

characterize their behavior against the response of the actual ECU with actuating and sensing devices. The results of the HIL test are shown in Figure 9.7, which confirms the results of the previous MIL and SIL results. For this experimental test, the parameters $\alpha$ exhibit some slow drift, probably caused by temperature variations in the valves. As expected, the tracking algorithm is able to follow these slow variations and preserve the reference tracking features of the scheme. 

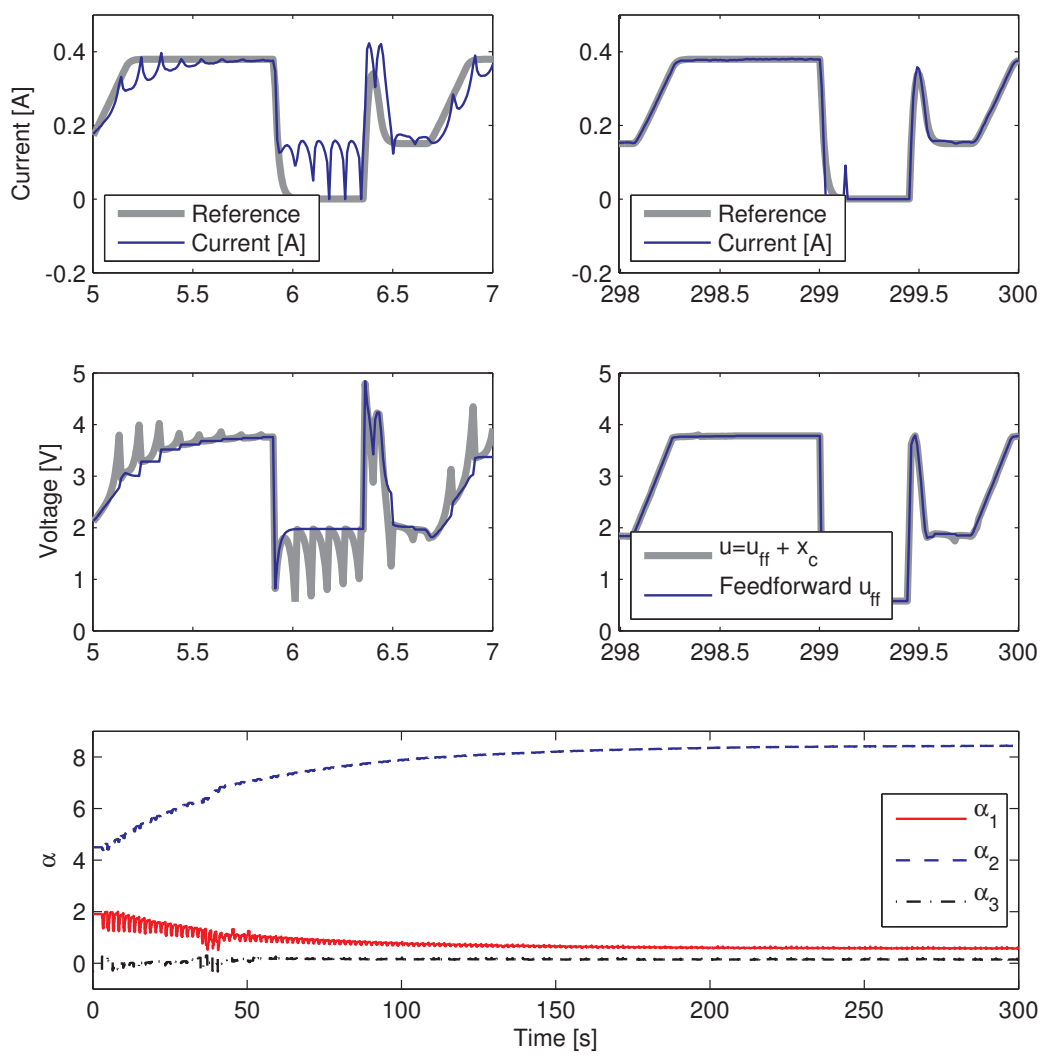

Figure 9.7: Experimental results with the HIL system. Two upper left plots: output currents (top) and input voltages (bottom) at the beginning of the adaptation. Two upper right plots: output currents (top) and input voltages (bottom) at the end of the adaptation. Down plot: time-evolution of the parameters $\alpha_{1}, \alpha_{2}$ and $\alpha_{3}$.

Finally, a different HIL experiment has been carried out by modifying the shape of the reference wave. In particular, we have used the sum of two sinusoidal waves with non commensurate periods, so that the arising signal is not periodic. The results, reported in Figure 9.8 illustrate once again the merits of the tracking algorithm and are associated to a correct convergence of the parameters $\alpha$ to the same values reached in the experiment of Figure 9.7. Observing Figure 9.8 one can appreciate that once the parameters have converged, very little action 

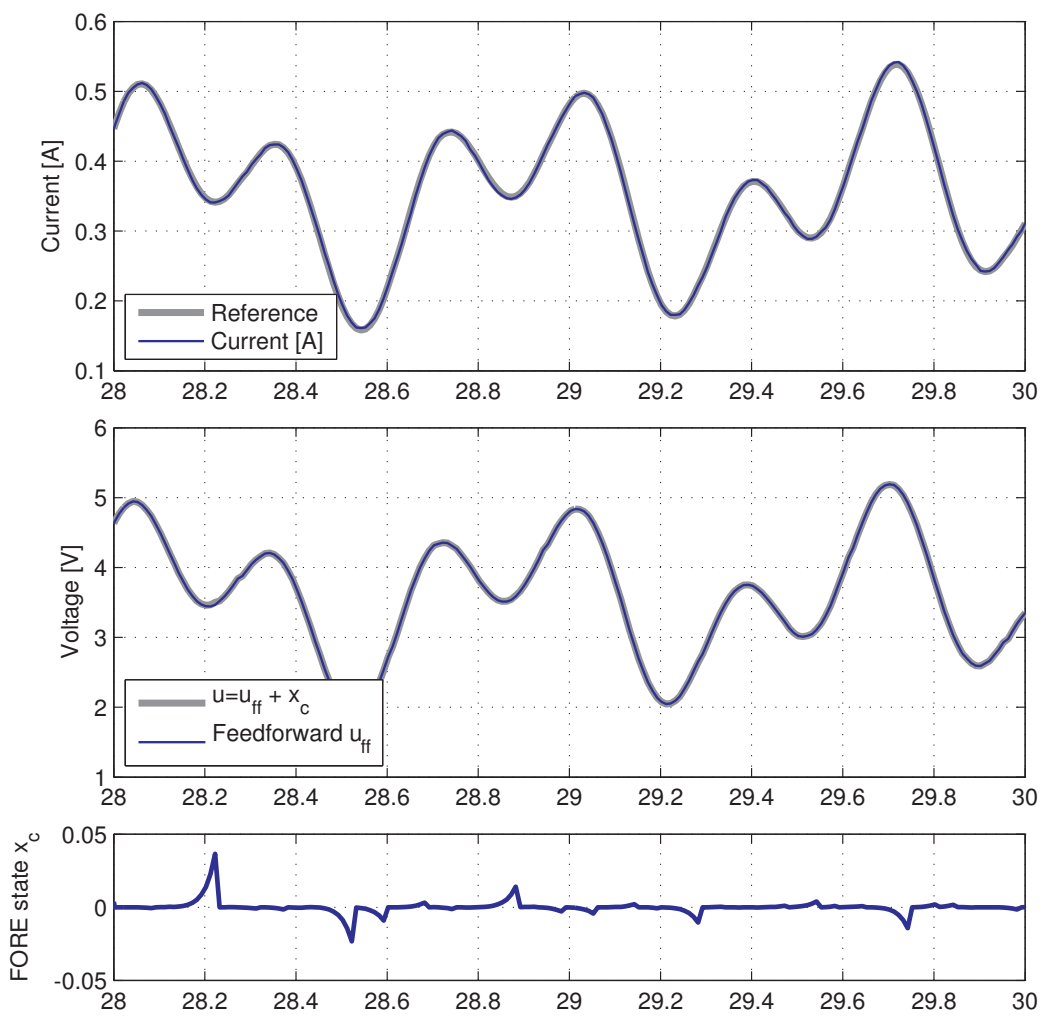

Figure 9.8: Experimental response to trigonometric reference.

is performed by the feedback part of the scheme (see the lowest plot). In addition, carefully inspecting the two upper traces of the figure, one realizes that the knowledge of the time derivative of the reference $\dot{r}$ enables a non-causal action at the plant input $u$ which appears to be a slightly anticipated (see, e.g. times 28.8 and 29.2) and distorted version of the actual reference $r$, capable of inducing perfect tracking. This result automatically arises from the effect of the linear combination in 7.23 for correct values of $\alpha=\alpha^{*}$. 


\section{Conclusions and perspectives}

\subsection{Conclusions}

Motivated by a linear analog integrator, namely the Clegg integrator circuit (see the description provided in Chapter 1), we introduced a particular class of controllers: the reset control laws. Such controllers have a mixed discrete/continuous dynamics, yielding to a hybrid dynamical closed-loop system when closing the loop with a continuous-time plant. The interest of such controllers has been presented and motivated by some examples (see in particular in Chapter 2). In particular, the additional discrete dynamics with respect to continuous-time dynamical controllers may allow to attain a better performance, e.g., in terms of the speed of convergence or in terms of the overshoot reduction.

This improvement has been precisely stated for nonlinear plants in Chapter 3, where design methods for state feedback laws have been presented. Using only a part of the state, and designing reset output feedback controllers is a challenging problem for both control theory and stability analysis. Indeed, the separation principle, that is classical in linear control theory, does not apply in general when closing the loop with output feedback reset laws. Moreover, as far as stability of hybrid dynamical systems is concerned, using observers may lead to the ex- 
istence of Zeno solutions, which are defective and should be avoided. These aspects motivated us to introduce, in Chapter 44 a temporal regularization written in terms of an additional reset dynamics providing a dwell time between consecutive jumps. Combining the results of the preceding chapters with this temporal regularization yielded design algorithms for output feedback reset controllers, mainly for linear continuous-time plants. Numerically tractable algorithms have been developed in Chapter 5 to estimate the $\mathcal{L}_{2}$ gain of the closed-loop system when studying the performance of measured outputs with respect to exogenous signals. The explicit computation of Lyapunov functions and of the estimation of the $\mathcal{L}_{2}$ gain was provided in terms of Linear Matrix Inequalities for Single-Input-Single-Output (SISO) linear plant controlled by First-Order-Reset-Elements (FORE). Further results on $\mathcal{H}_{\infty}$ control design have been given in Chapter 6, where again the full state availability and the partial state availability (output) cases are studied separately. Illustrative examples have been given on physical models of real applications by exhibiting both the advantages and drawbacks of the results.

Planar reset systems have been studied in the last part of this survey. First further results were given on planar SISO linear plants controlled by FORE controllers, paralleling the results of Chapter 5 when focusing to planar plants. Advanced results were given to solve set-point and trajectory tracking problems in Chapter 7. The obtained results were applied to the control of Exhaust Gas Recirculation (EGR) by FORE in Chapter 8 where, after an identification procedure, adaptive feedforward FORE were implemented on a Diesel engine testbench. FORE control was applied to an electromechanical valve in a traction drive device in Chapter 9. Experimental tests illustrated the merits of the tracking algorithm.

\subsection{Perspectives}

The central approach, presented in this survey on reset controllers, has many connections with other results and works on control theory, and on engineering applications of automatic control. To cite a few 
results, consider the important works on design of hybrid controllers for nonlinear plants, in particular for control systems where stabilizing continuous dynamical controllers do not exist, as considered in Liberzon [2003], Prieur and Astolfi [2003], Prieur and Teel [2011] among other references. It could be useful to revisit this literature on the design of hybrid controllers and to apply the techniques presented in this paper for the design of robust reset controllers. The use of timers and dwell time is important when using observers in the closed-loop system as it has been seen in this survey for linear control systems. It is also important for nonlinear ones. See in particular Marx et al. 2016] where dwell-time property has been employed to combine a high-gain observer with a hybrid controller for general nonlinear control systems.

Many results for the design of event-triggered controllers are related to the results and methods presented in this survey. For both linear plants and nonlinear models, designing event-triggered controllers may reduce the need for input updates when controlling a plant, and may also reduce the need for measuring the plant outputs. This is particularly important for systems measured and controlled through networks (as considered in Tabuada [2007], Postoyan et al. 2011], Peng and Yang [2013]). Different event-triggered schemes exist, in particular the periodic approach (see Heemels et al. 2013] ) for linear plants, and Lyapunov based techniques for output stabilization of nonlinear systems (as in Tanwani et al. 2015, Abdelrahim et al. 2017]). Reset controllers (and more generally hybrid controllers) could be applied when the information is quantized in the networks. See the recent works Ferrante et al. 2015, Tanwani et al. 2016 on this subject. Reset control techniques are also fruitful for controlling plants where only sporadic measures and inputs are available, as considered in Ferrante et al. [2016].

Reset controllers may also be applied when controlling plants with a saturating input. They allow to enlarge the basin of attraction of the saturated control system (see Loquen et al. [2007]), and to solve a tracking reference problem in presence of input saturation, as done in Loquen et al. [2008. Dedicated stability analysis and anti-windup techniques exist for reset control systems, as studied respectively in Fiacchini et al. 2012, 2013 and in Tarbouriech et al. [2011]. Connections 
of reset controllers with delay is also an interesting research line, see Baños et al. 2014b for a recent work on stability results when delay effect is considered.

In summary, this survey paves the way for future directions of research including taking into account in a generic fashion the isolated or structural nonlinearities affecting the system (as, for example, saturation) to improve the characterization of the basin of attraction of the closed loop. The design of reset controllers to overcome several limitations of classical continuous-time systems as the presence of uncertainties or delay could be also fruitful. Moreover, the use of reset controllers to develop new paradigms in networked control systems could be very interesting. 


\section{Acknowledgement}

The authors would like to sincerely thank the many collaborators with whom much of the results reported here have been developed.

First and foremost we would like to thank Dragan Nešić, for his visionary ideas back in 2004, which attracted the attention of us all towards this very interesting research field. Special thanks go to Andy Teel who trained us to his view of Lyapunov theory for nonlinear hybrid dynamical systems. Without those results, most of the developments in this survey would have not been possible. We are also grateful to Thomas Loquen, the $\mathrm{PhD}$ student who witnessed and favored the beginning of the scientific interaction among the authors of this survey, and then Francesco Fichera who diligently and tirelessly developed with us many of the results reported in part II of this book.

We would like to thank Alfonso Baños and Antonio Barreiro for the useful exchanges arising from the long-term visits at the LAAS-CNRS of their PhD students Miguel A. Davó, José Antonio González Prieto, and Félix Pérez Rubio. We would also like to thank other students and collaborators with whom we discussed and developed several aspects of reset control: Matteo Cocetti, Mirko Fiacchini, Fulvio Forni, Laurentiu Hetel, and Alexandre Seuret. Finally, we would like to thank 
the people allowing us to actually experiment some of the developed FORE solutions, as discussed in part III of the book: Francesco Saverio Panni, Daniel Alberer, Harald Waschl who made the EGR application of Chapter 8 possible, and also Matteo Cordioli, Fabrizio Panizzolo, Pier Paolo Rinaldi and Alex Sassaro from Dana Rexroth Transmission Systems, who were a key towards the development of the work of Chapter 9

Last but not least our thanks go to Panos Antsaklis and Alessandro Astolfi for motivating us to write this survey, and for their invaluable patience in waiting for us to finally find the time and the energy to write it and submit it to this journal.

Furthermore, this work was partially supported by the ANR Projects LIMICOS, contract number 12BS0300501, and SCIDIS, contract number 15-CE23-0014, by the University of Trento, grant OptHySYS, by the CaRiTrO and DRTS through grant PowerLyap. Then we thank these agencies for their financial support. 


\section{References}

W. Aangenent, G. Witvoet, W. Heemels, MJG van de Molengraft, and M. Steinbuch. An LMI-based $\mathcal{L}_{2}$ performance analysis for reset control systems. In American Control Conference, pages 2248-2253, Seattle (WA), USA, 2008.

W. Aangenent, G. Witvoet, W. Heemels, MJG van de Molengraft, and M. Steinbuch. Performance analysis of reset control systems. International Journal of Robust and Nonlinear Control, 20(11):1213-1233, 2010.

M. Abdelrahim, R. Postoyan, J. Daafouz, and D. Nešić. Robust eventtriggered output feedback controllers for nonlinear systems. Automatica, 75:96-108, 2017.

V. Andrieu, C. Prieur, S. Tarbouriech, and L. Zaccarian. A hybrid scheme for reducing peaking in high-gain observers for a class of nonlinear systems. Automatica, pages 138-146, 2016.

A. Anta and P. Tabuada. To sample or not to sample: self-triggered control for nonlinear systems. IEEE Transactions on Automatic Control, 55:2030$2042,2010$.

Z. Artstein. Stabilization with relaxed controls. Nonlinear Anal., 7(11):11631173, 1983.

J. Bakkeheim, T.A. Johansen, Ø.N. Smogeli, and A.J. Sørensen. Lyapunovbased integrator resetting with application to marine thruster control. IEEE Transactions on Control Systems Technology, 16(5):908-917, 2008.

A. Baños and A. Barreiro. Delay-independent stability of reset systems. IEEE Transactions on Automatic Control, 54(2):341-346, 2009. 
A. Baños and A. Barreiro. Reset control systems. Springer, 2011.

A. Baños, F. Perez, and J. Cervera. Network-based reset control systems with time-varying delays. IEEE Transactions on Industrial Informatics, 10(1): 514-522, 2014a.

A. Baños, F. P. Rubio, S. Tarbouriech, and L. Zaccarian. Delay-independent stability via reset loops. In Low-Complexity Controllers for Time-Delay Systems, pages 111-125. Springer, 2014b.

A. Barreiro and A. Baños. Delay-dependent stability of reset systems. Automatica, 46:216-221, 2010.

A. Barreiro, A. Baños, S. Dormido, and J.A. González-Prieto. Reset control systems with reset band: Well-posedness, limit cycles and stability analysis. Systems \& Control Letters, 63:1-11, 2014.

O. Beker, C.V. Hollot, and Y. Chait. Stability of a MIMO reset control system under constant inputs. In IEEE Conference on Decision and Control, pages 2780-2781, Phoenix, Arizona, 1999a.

O. Beker, C.V. Hollot, Q. Chen, and Y. Chait. Stability of a reset control system under constant inputs. In American Control Conference, pages 3044-3045, San Diego, California, 1999b.

O. Beker, C.V. Hollot, and Y. Chait. Plant with integrator: an example of reset control overcoming limitations of linear feedback. IEEE Transactions on Automatic Control, 46(11):1797-1799, 2001a.

O. Beker, C.V. Hollot, and Y. Chait. Stability of limit cycles in reset control systems. In American Control Conference, pages 4681-4682, Arlington, Virginia, 2001b.

O. Beker, C.V. Hollot, Y. Chait, and H. Han. Fundamental properties of reset control systems. Automatica, 40(6):905-915, 2004.

F. Blanchini and C. Savorgnan. Stabilizability of switched linear systems does not imply the existence of convex lyapunov functions. In IEEE Conference on Decision and Control, pages 119-124. IEEE, 2006.

S.P. Boyd, L. El Ghaoui, E. Feron, and V. Balakrishnan. Linear Matrix Inequalities in System and Control Theory. Society for Industrial and Applied Mathematics, 1994.

R.T. Bupp, D.S. Bernstein, V.S. Chellaboina, and W.M. Haddad. Resetting virtual absorbers for vibration control. Journal of Vibration and Control, $6(1): 61-83,2000$.

C. Cai and A.R. Teel. Characterizations of Input-to-state Stability for Hybrid Systems. Systems \& Control Letters, 58(1):47-53, 2009. 
C. Cai, A.R. Teel, and R. Goebel. Smooth Lyapunov Functions for Hybrid Systems, Part II: (Pre)Asymptotically Stable Compact Sets. IEEE Transactions on Automatic Control, 53(3):734-748, 2008.

J. Carrasco and A. Baños. Reset control of an industrial in-line pH process. IEEE Transactions on Control Systems Technology, 20(4):1100-1106, 2012.

J. Carrasco, A. Baños, and A. van der Schaft. A passivity-based approach to reset control systems stability. Systems \& Control Letters, 59:18-24, 2010.

Y. Chait and C.V. Hollot. On Horowitz's contributions to reset control. Int. J. Rob. Nonlin. Contr., 12:335-355, 2002.

Q. Chen, C.V. Hollot, and Y. Chait. Stability and asymptotic performance analysis of a class of reset control systems. In IEEE Conference on Decision and Control, pages 251-256, Sidney, Australia, 2000a.

Q. Chen, C.V. Hollot, Y. Chait, and O. Beker. On reset control systems with second order plants. In American Control Conference, pages 205-209, Chicago, Illinois, 2000b.

Q. Chen, Y. Chait, and C.V. Hollot. Analysis of reset control systems consisting of a fore and second order loop. J. Dynamic Systems, Measurement and Control, 123:279-283, 2001.

M. Chilali and P. Gahinet. $H_{\infty}$ Design with Pole Placement Constraints: an LMI Approach. IEEE Transactions on Automatic Control, 41:358-367, 1996.

F.H. Clarke. Optimization and Nonsmooth Analysis. Society for Industrial and Applied Mathematics, 1990.

J.C. Clegg. A nonlinear integrator for servomechanisms. Trans. A. I. E. E., 77 (Part II):41-42, 1958.

M. Cordioli. Hybrid current controller for proportional electro-hydraulic pressure reducing valve. MS thesis, University of Trento, Italy, 2014.

M. Cordioli, M. Mueller, F. Panizzolo, F. Biral, and L. Zaccarian. An adaptive reset control scheme for valve current tracking in a power-split transmission system. In European Control Conference, pages 1878-1883, Linz, Austria, July 2015.

M.A. Davó, A. Baños, F. Gouaisbaut, S. Tarbouriech, and A. Seuret. Stability analysis of linear impulsive delay dynamical systems via looped-functionals. Automatica, 81:107-114, 2017.

A. Fernandez, A. Barreiro, A. Banos, and J. Carrasco. Reset control for passive teleoperation. In 34th Annual Conference of IEEE Industrial Electronics, pages 2935-2940, 2008. 
F. Ferrante, F. Gouaisbaut, and S. Tarbouriech. Stabilization of continuoustime linear systems subject to input quantization. Automatica, 58:167-172, 2015 .

F. Ferrante, F. Gouaisbaut, R.G. Sanfelice, and S. Tarbouriech. State estimation of linear systems in the presence of sporadic measurements. Automatica, 73:101-109, 2016.

A. Feuer, G.C. Goodwin, and M. Salgado. Potential benefits of hybrid control for linear time invariant plants. In American Control Conference, pages 2790-2794, Albuquerque, New Mexico, 1997.

M. Fiacchini, S. Tarbouriech, and C. Prieur. Quadratic stability for hybrid systems with nested saturations. IEEE Transactions on Automatic Control, $57(7): 1832-1838,2012$.

M. Fiacchini, S. Tarbouriech, and C. Prieur. Exponential stability for hybrid systems with saturations. Hybrid systems with constraints, pages 179-212, 2013.

F. Fichera. Lyapunov techniques for a class of hybrid systems and reset controller syntheses for continuous-time plants. PhD thesis, ISAE, Toulouse, October 2013.

F. Fichera, C. Prieur, S. Tarbouriech, and L. Zaccarian. Improving the Performance of Linear Systems by Adding a Hybrid Loop: the Output Feedback Case. In American Control Conference, Montreal, Canada, 2012a.

F. Fichera, C. Prieur, S. Tarbouriech, and L. Zaccarian. On Hybrid Statefeedback Loops Based on a Dwell-time Logic. In 4th IFAC Conference on Analysis and Design of Hybrid Systems, Eindoven, The Netherlands, 2012b.

F. Fichera, C. Prieur, S. Tarbouriech, and L. Zaccarian. A convex hybrid $\mathcal{H}_{\infty}$ synthesis with guaranteed convergence rate. In IEEE Conference on Decision and Control, pages 4217-4222, Maui (HI), USA, 2012c.

F. Fichera, C. Prieur, S. Tarbouriech, and L. Zaccarian. Using Luenberger observers and dwell-time logic for feedback hybrid loops in continuous-time control systems. International Journal of Robust and Nonlinear Control, 23:1065-1086, 2013.

F. Fichera, C. Prieur, S. Tarbouriech, and L. Zaccarian. LMI-based Reset $\mathcal{H}_{\infty}$ Analysis and Design for Linear Continuous-time Plants. $H A L$, hal-number: hal-01236972v1, 2015.

F. Fichera, C. Prieur, S. Tarbouriech, and L. Zaccarian. LMI-based reset h-infinity design for linear continuous-time plants. IEEE Transactions on Automatic Control, 61(12):4157-4163, 2016. 
F. Forni, S. Galeani, D. Nešić, and L. Zaccarian. Lazy Sensors for the Scheduling of Measurements Samples Transmission in Linear Closed Loops Over Networks. In IEEE Conference on Decision and Control, pages 6469-6474, Atlanta (GA), USA, December 2010.

F. Forni, D. Nešić, and L. Zaccarian. Reset Passivation of Nonlinear Controllers via Suitable Time-regular Reset Map. Automatica, 47(9):2099-2106, 2011.

F. Forni, S. Galeani, D. Nešić, and L. Zaccarian. Event-triggered transmission for linear control over communication channels. Automatica, 50(2):490-498, 2014.

G. Franklin, J.D. Powell, and A. Emami-Naeini. Feedback Control of Dynamic Systems, 6Ed. Prentice Hall, 2009.

R. A. Freeman and P. V. Kokotović. Inverse optimality in robust stabilization. SIAM J. Control Optim., 34(4):1365-1391, 1996.

V. Ghaffari, P. Karimaghaee, and A. Khayatian. Reset law design based on robust model predictive strategy for uncertain systems. Journal of Process Control, 24(1):261-268, 2014.

R. Goebel and A.R. Teel. Solutions to hybrid inclusions via set and graphical convergence with stability theory applications. Automatica, 42(4):573-587, 2006.

R. Goebel and A.R. Teel. Preasymptotic stability and homogeneous approximations of hybrid dynamical systems. SIAM Review, 52(1):87-109, 2010.

R. Goebel, J. Hespanha, A.R. Teel, C. Cai, and R.G. Sanfelice. Hybrid systems: Generalized solutions and robust stability. In IFAC Symposium on Nonlinear Control Systems, pages 1-12, Stuttgart, Germany, 2004.

R. Goebel, R.G. Sanfelice, and A.R. Teel. Hybrid Dynamical Systems. IEEE Control Systems Magazine, 29(2):28-93, April 2009.

R. Goebel, R. G. Sanfelice, and A. R. Teel. Hybrid Dynamical Systems: Modeling, Stability, and Robustness. Princeton University Press, 2012.

Y. Guo, Y. Wang, L. Xie, and J. Zheng. Stability analysis and design of reset systems: Theory and an application. Automatica, 45(2):492-497, 2009.

Y. Guo, W. Gui, C. Yang, and L. Xie. Stability analysis and design of reset control systems with discrete-time triggering conditions. Automatica, 48 (3):528-535, 2012.

W.M. Haddad, V. Chellaboina, and N. Kablar. Active control of combustion instabilities via hybrid resetting controllers. In American Control Conference, pages 2378-2382, Chicago, Illinois, 2000. 
W.M. Haddad, V. Chellaboina, and N. Kablar. Non-linear impulsive dynamical systems. Part II: Stability of feedback interconnections and optimality. International Journal of Control, 74(17):1659-1677, 2001.

W.P.M.H. Heemels, M.C.F. Donkers, and A.R. Teel. Periodic event-triggered control for linear systems. IEEE Transactions on Automatic Control, 58: 847-861, 2013.

W.P.M.H. Heemels, G.E. Dullerud, and A.R. Teel. $\mathcal{L}_{2}$-gain analysis for a class of hybrid systems with applications to reset and event-triggered control: A lifting approach. IEEE Transactions on Automatic Control, 61(10):27662781, 2016.

L. Hetel, J. Daafouz, S. Tarbouriech, and C. Prieur. Stabilization of linear impulsive systems through a nearly-periodic reset. Nonlinear Analysis: Hybrid Systems, 7(1):4-15, 2013.

C.V. Hollot, Y. Zheng, and Y. Chait. Stability analysis for control systems with reset integrators. In IEEE Conference on Decision and Control, pages 1717-1719, San Diego, California, 1997.

C.V. Hollot, O. Beker, Y. Chait, and Q. Chen. On establishing classic performance measures for reset control systems. In S.O. Moheimani, editor, Perspectives in robust control, LNCIS 268, pages 123-147. Springer, 2001.

I. Horowitz and P. Rosenbaum. Non-linear design for cost of feedback reduction in systems with large parameter uncertainty. International Journal of Control, 21:977-1001, 1975.

H. Hu, Y. Zheng, Y. Chait, and C.V. Hollot. On the zero inputs stability of control systems with clegg integrators. In American Control Conference, pages 408-410, Albuquerque, New Mexico, 1997.

A. Isidori. Nonlinear control systems. Communications and Control Engineering Series. Springer-Verlag, Berlin, third edition, 1995.

K.H. Johansson, J. Lygeros, S. Sastry, and M. Egerstedt. Simulation of Zeno hybrid automata. In IEEE Conference on Decision and Control, pages 3538-3543, Phoenix, Arizona, 1999.

P. Kapasouris, M. Athans, and G. Stein. Design of Feedback Control Systems for Stable Plants with Saturating Actuators. In IEEE Conference on Decision and Control, pages 469-479, Austin, TX, 1988.

H.K. Khalil. Nonlinear Systems. Prentice-Hall, 3rd edition, 2002.

K.R. Krishnan and I.M. Horowitz. Synthesis of a non-linear feedback system with significant plant-ignorance for prescribed system tolerances. Int. J. Contr., 19:689-706, 1974. 
M. Krstić, I. I. Kanellakopoulos, and P. V. Kokotović. Nonlinear and Adaptive Control Design. Communications and Control Engineering Series. Wiley, New York, 1995.

H. Li, C. Du, Y. Wang, and Y. Guo. Discrete-time optimal reset control for hard disk drive servo systems. IEEE Transactions on Magnetics, 45(11 Part 1):5104-5107, 2009.

Y. Li, G. Guo, and Y. Wang. Reset control for midfrequency narrowband disturbance rejection with an application in hard disk drives. IEEE Transactions on Control Systems Technology, 19(6):1339-1348, 2011.

D. Liberzon. Switching in systems and control. Springer, 2003.

Y. Lin and E.D. Sontag. A universal formula for stabilization with bounded controls. Systems \&3 Control Letters, 16(6):393-397, 1991.

L. Ljung. System Identification Toolbox for Use with MATLAB. The Mathworks, Inc., 2007.

J. Löfberg. YALMIP : A Toolbox for Modeling and Optimization in MATLAB. In Proceedings of the CACSD Conference, Taipei, Taiwan, 2004. URL http://users.isy.liu.se/johanl/yalmip.

T. Loquen, S. Tarbouriech, and C. Prieur. Stability analysis for reset systems with input saturation. In IEEE Conference on Decision and Control, pages 3272-3277, New Orleans, LA, USA, 2007.

T. Loquen, S. Tarbouriech, and C. Prieur. Stability of reset control systems with nonzero reference. In IEEE Conference on Decision and Control, pages 3386-3391, Cancun, Mexico, 2008.

D.G. Luenberger. Observers for Multivariable Systems. IEEE Transactions on Automatic Control, 11(2):190-197, 1966.

S. Marx, V. Andrieu, and C. Prieur. Semi-global stabilization by an output feedback law from a hybrid state controller. Automatica, 74:90-98, 2016.

M. Mueller, W. Scandella, and S. Mutschler. Power-split transmission for a traction drive and method for controlling the transmission, August 21 2014. URL http://www.google.com/patents/US20140236433. US Patent Application 14/182,584.

D. Nešić, L. Zaccarian, and A.R. Teel. Stability properties of reset systems. In IFAC World Congress, Prague, Czech Republic, 2005.

D. Nešić, A.R. Teel, and L. Zaccarian. On necessary and sufficient conditions for exponential and $\mathcal{L}_{2}$ stability of planar reset systems. In American Control Conference, pages 4140-4145, Seattle (WA), USA, June 2008a. 
D. Nešić, L. Zaccarian, and A.R. Teel. Stability properties of reset systems. Automatica, 44(8):2019-2026, 2008b.

D. Nešić, A. R. Teel, and L. Zaccarian. Stability and performance of SISO control systems with First Order Reset Elements. IEEE Transactions on Automatic Control, 56(11):2567-2582, 2011.

D. Nešić, A.R. Teel, G. Valmorbida, and L. Zaccarian. Finite-Gain $\mathcal{L}_{p}$ Stability for Hybrid Dynamical Systems. Automatica, 49(8):2384-2396, 2013.

S. Nicosia, A. Tornambè, and P. Valigi. Experimental results in state estimation of industrial robots. In IEEE Conference on Decision and Control, pages 360-365, December 1990.

D. Paesa, A. Baños, and C. Sagues. Optimal reset adaptive observer design. Systems \& Control Letters, 60:877-883, 2011.

D. Paesa, C. Franco, S. Llorente, G. Lopez-Nicolas, and C. Sagues. Reset adaptive observer for a class of nonlinear systems. IEEE Transactions on Automatic Control, 57(2):506-511, 2012.

F.S. Panni, H. Waschl, D. Alberer, and L. Zaccarian. Position regulation of an EGR valve using reset control with adaptive feedforward. IEEE Transactions on Control Systems Technology, 22(6):2424-2431, 2014.

C. Peng and T.C. Yang. Event-triggered communication and $H_{\infty}$ control codesign for networked control systems. Automatica, 49(5):1326-1332, 2013.

L. S. Pontryagin, V. G. Boltyanskij, R. V. Gamkrelidze, and E. F. Mishchenko. The mathematical theory of optimal processes. Wiley, 1962.

R. Postoyan, P. Tabuada, D. Nesic, and A. Anta. Event-triggered and selftriggered stabilization of distributed networked control systems. In IEEE Conference on Decision and Control and European Control Conference, pages 2565-2570, Orlando, USA, 2011.

C. Prieur and A. Astolfi. Robust stabilization of chained systems via hybrid control. IEEE Transactions on Automatic Control, 48(10):1768-1772, 2003.

C. Prieur and A.R. Teel. Uniting local and global output feedback controllers. IEEE Transactions on Automatic Control, 56(7):1636-1649, 2011.

C. Prieur, R. Goebel, and A. R. Teel. Hybrid feedback control and robust stabilization of nonlinear systems. IEEE Transactions on Automatic Control, 52(11):2103-2117, 2007.

C. Prieur, S. Tarbouriech, and L. Zaccarian. Guaranteed stability for nonlinear systems by means of a hybrid loop. In IFAC Symposium on Nonlinear Control Systems, pages 72-77, Bologna, Italy, September 2010. 
C. Prieur, S. Tarbouriech, and L. Zaccarian. Improving the performance of linear systems by adding a hybrid loop. In 18th IFAC World Congress, pages 6301-6306, Milan, Italy, 2011.

C. Prieur, S. Tarbouriech, and L. Zaccarian. Lyapunov-based hybrid loops for stability and performance of continuous-time control systems. Automatica, 49(2):577-584, 2013.

H. Rios, L. Hetel, and D. Efimov. Nonlinear impulsive systems: 2D stability analysis approach. Automatica, 80:32-40, 2017.

R. Sanfelice, D. Copp, and P. Nanez. A toolbox for simulation of hybrid systems in Matlab/Simulink: hybrid equations (HyEQ) toolbox. In Proceedings of the 16th international conference on Hybrid systems: computation and control. ACM, 2013.

A. Satoh. State feedback synthesis of linear reset control with $\mathcal{L}_{2}$ performance bound via LMI approach. In 18th IFAC World Congress, pages 5860-5865, Milan, Italy, 2011.

C. Scherer, P. Gahinet, and M. Chilali. Multiobjective Output-feedback Control via LMI Optimization. IEEE Transactions on Automatic Control, 42 (7), 1997.

A. Seuret, C. Prieur, and N. Marchand. Stability of non-linear systems by means of event-triggered sampling algorithms. IMA Journal of Mathematical Control and Information, 31(3):415-433, 2014.

A. Seuret, S. Tarbouriech, C. Prieur, and L. Zaccarian. Event-triggered control via reset control systems framework. In IFAC Symposium on Nonlinear Control Systems, Monterey, CA, USA, 2016.

P. Tabuada. Event-triggered real-time scheduling of stabilizing control tasks. IEEE Transactions on Automatic Control, 52(9):1680-1685, 2007.

A. Tanwani, A.R. Teel, and C. Prieur. On using norm estimators for eventtriggered control with dynamic output feedback. In IEEE Conference on Decision and Control, Osaka, Japan, 2015.

A. Tanwani, C. Prieur, and M. Fiacchini. Observer-based feedback stabilization of linear systems with event-triggered sampling and dynamic quantization. Systems \& Control Letters, 94:46-56, 2016.

S. Tarbouriech, T. Loquen, and C. Prieur. Anti-windup strategy for reset control systems. International Journal of Robust and Nonlinear Control, 21(10):1159-1177, 2011.

A.R. Teel. Observer-based hybrid feedback: a local separation principle. In American Control Conference, pages 898-903, June 2010. 
A.R. Teel, F. Forni, and L. Zaccarian. Lyapunov-based sufficient conditions for exponential stability in hybrid systems. IEEE Transactions on Automatic Control, 58(6):1591-1596, 2013.

S.E. Tuna and A.R. Teel. Homogeneous hybrid systems and a converse Lyapunov theorem. In 45th IEEE Conference on Decision and Control, 2006, pages 6235-6240, December 2006.

S.J.L.M. van Loon, K.G.J. Gruntjens, M.F. Heertjes, N. van de Wouw, and W.P.M.H. Heemels. Frequency-domain tools for stability analysis of reset control systems. Automatica, 82:101-108, 2017.

A. Vidal and A. Baños. Reset compensation for temperature control: Experimental application on heat exchangers. Chemical Engineering Journal, 159 (1-3):170-181, 2010.

A.F. Villaverde, A. Barreiro Blas, J. Carrasco, and A. Baños Torrico. Reset control for passive bilateral teleoperation. IEEE Transactions on Industrial Electronics, 58(7):3037-3045, 2011.

G. Witvoet, W. Aangenent, W. Heemels, MJG van de Molengraft, and M. Steinbuch. $\mathcal{H}_{2}$ performance analysis of reset control systems. In Conference on Decision and Control, pages 3278-3284, New Orleans (LA), USA, 2007.

D. Wu, G. Guo, and Y. Wang. Reset integral-derivative control for HDD servo systems. IEEE Transactions on Control Systems Technology, 15(1): 161-167, 2007.

C. Yuan and F. Wu. Output feedback reset control of general MIMO LTI systems. In European Control Conference, pages 2334-2339, Strasbourge, france, June 2014.

L. Zaccarian, D. Nešić, and A. R. Teel. First order reset elements and the Clegg integrator revisited. In American Control Conference, pages 563-568, Portland (OR), USA, June 2005.

L. Zaccarian, D. Nešić, and A.R. Teel. Explicit Lyapunov functions for stability and performance characterizations of FOREs connected to an integrator. In IEEE Conference on Decision and Control, pages 771-776, San Diego (CA), USA, December 2006.

L. Zaccarian, D. Nešić, and A.R. Teel. Set-point stabilization of SISO linear systems using First Order Reset Elements. In American Control Conference, pages 5808-5809, New York (NY), USA, July 2007. 
L. Zaccarian, D. Nešić, and A.R. Teel. Analytical and Numerical Lyapunov Functions for SISO Linear Control Systems with First-order Reset Elements. International Journal of Robust and Nonlinear Control, 21:11341158, 2011.

G. Zhao and C. Hua. Improved high-order-reset-element model based on circuit analysis. IEEE Transactions on Circuits and Systems - II, 64(4): 432-436, April 2017.

G. Zhao and J. Wang. Reset observers for linear time-varying delay systems: Delay-dependent approach. Journal of the Franklin Institute, 351(11):51335147, 2014.

J. Zheng, Y. Guo, M. Fu, Y. Wang, and L. Xie. Improved reset control design for a PZT positioning stage. In IEEE International Conference on Control Applications, pages 1272-1277, 2007.

Y. Zheng, Y. Chait, C.V. Hollot, M. Steinbuch, and M. Norg. Experimental demonstration of reset control design. Control Engineering Practice, 8: 113-120, 2000. 